Portland State University

PDXScholar

Summer 7-26-2016

\title{
Bridging the Worlds of Home and School: a Study of the Relational Worlds of First-Generation Students in a School of Social Work
}

Miranda Cunningham

Portland State University

Follow this and additional works at: https://pdxscholar.library.pdx.edu/open_access_etds

Part of the Social Work Commons, and the Sociology Commons Let us know how access to this document benefits you.

Recommended Citation

Cunningham, Miranda, "Bridging the Worlds of Home and School: a Study of the Relational Worlds of FirstGeneration Students in a School of Social Work" (2016). Dissertations and Theses. Paper 3088.

https://doi.org/10.15760/etd.3083

This Dissertation is brought to you for free and open access. It has been accepted for inclusion in Dissertations and Theses by an authorized administrator of PDXScholar. Please contact us if we can make this document more accessible: pdxscholar@pdx.edu. 
Bridging the Worlds of Home and School:

A Study of the Relational Worlds of First-Generation Students

in a School of Social Work

by

Miranda Joyce Cunningham

A dissertation submitted in partial fulfillment of the requirements for the degree of

Doctor of Philosophy

in

Social Work and Social Research

Dissertation Committee:

Ben Anderson-Nathe, Chair

Stéphanie Wahab

Katharine Cahn

Anita Bright

Portland State University

2016 
(C) 2016 Miranda Joyce Cunningham 


\begin{abstract}
Much scholarship on first-generation students has focused on their academic and social integration in college (Collier \& Morgan, 2008; Lowery-Hart \& Pacheco, 2011; Stuber, 2011). Little is known about the experiences of first-generation students in schools of social work. In this research I've expanded the focus beyond students' experiences of academic integration to explore how first-generation students in a school of social work describe their relational worlds and the implications for professional socialization.
\end{abstract}

Informed by Standpoint Feminism and Postmodern/Post structural Feminism, I conducted focus groups with 19 students in two undergraduate programs and one graduate program in a school of social work and analyzed these conversations using Voice-centered Relational Data Analysis (Brown \& Gilligan, 1992). This research highlighted how students bridge the cultures of home and school through 1) Experiences of support from home cultures while 2) pursuing school largely on their own and experiencing 3) the potential for distance from cultures of home, as they 4) work to stay integrated in home cultures while simultaneously 5) working to become integrated in school. I've also written about students' experiences of becoming caught "in-between" the cultures of home and school (Anzaldúa, 1987/2012), a less common but nevertheless important experience for educators to attend to.

Here I've argued for broadening the focus beyond academic integration (Tinto, 1975, 1993) and underscored the relational nature of first-generation status, as well as drawing attention to potential for relational injury embedded in our narratives about 
educational attainment and class mobility. Implications for social work education, practice, and research are discussed. 


\section{ACKNOWLEDGEMENTS}

This dissertation would not have been possible without tremendous support from colleagues, friends, family, and students. Following is my attempt to note some of these many contributions.

First I want to thank the students who participated in these conversations.

Throughout analysis I often found myself pushing "pause" on the recording to reflect on your words. Some of your stories resonated deeply with my own experiences as a firstgeneration student and other stories were vastly different from my own, but the process was always enriching. Thank you for the gift of time and your selves.

I'm also thankful to my dissertation committee. Each of you has provided a critical balance of challenge and support throughout this process. I would not have made it beyond my first year in the doctoral program were it not for Dr. Stéphanie Wahab. I'm grateful for your brilliant advice on research design and analysis and insightful emotional support. Thank you as well to Dr. Katharine Cahn, who has shown me a model of leadership committed to emancipatory work and increasing equity. I'd also like to thank Dr. Anita Bright, my OGS representative. Finding an OGS representative presented one of the more challenging parts of this process to me, and it took less than a minute of conversation to realize Anita was the one! You embody gentleness and wisdom, and I'm so glad to have had you on my committee. Finally, I'm thankful to Dr. Ben AndersonNathe, who has supported me in many ways over the past few years: as committee chair, as a supervisor and teaching mentor, and as a dear friend. Some of my favorite memories 
as a PhD student will be sitting in conversations with you that challenged my assumptions, welcomed complexity, and were brimming with "a-ha!" moments.

During my time in the $\mathrm{PhD}$ program we've undergone tremendous changes, so I'm thankful for the constants, especially the unfailing kindness of our program assistants: Pauline Duffy, Jenny Robe, and Thet Mar Win. I'm also thankful for the guidance of Dr. Joan Shireman, Professor Emerita. I look forward to enjoying our first cup of coffee together as a fellow PhD. I'm also grateful for the School of Social Work's on-going support through graduate assistantships in the Regional Research Institute and teaching. During the writing of this dissertation I've also received funding from the Laurels Graduate tuition remission and the Longres Dissertation Fellowship; this funding was instrumental in allowing me to finish.

I'm also thankful to my many colleagues in the School of Social Work who've shared their dissertation stories and normalized some of my experiences over the past 18 months, stepping into a faculty position while completing this work. I am thankful for colleagues who have helped me navigate the transition from student to faculty (and back, as a doctoral candidate), especially the faculty members of the Monday writing group who encouraged me to join. I'm thankful for fellow doctoral students who have moved through this process with me, particularly Dr. Joseph DeFilippis, whose de-briefing and writing sessions kept me going, along with plenty of music and fried chicken. I'd also like to thank Dr. Roberta Hunte for her energy and encouragement (someday I hope to institutionalize Writers Uniting!). I'm also grateful to colleagues who showed interest in my work and invited me to your classrooms: Dr. Ericka Kimball, Dr. Gita Mehrotra, Dr. 
Jana Meinhold, and Kate Constable, and for Janet Putnam and Melissa Penners for providing demographic data and support interpreting complicated program schedules. Finally, I'm thankful for my employment in the School of Social Work, which has allowed me the financial stability to complete my dissertation and to my colleagues in Child and Family Studies, who share my enthusiasm for teaching and interdisciplinary work. I'm also grateful for our students, who make me happy to come to work each day.

I also want to thank the teachers who I met in my undergraduate education who set me on this path. I'm thankful to Dr. Susan Finley for the introduction to Paulo Freire, and Dr. Armando Estrada and Dr. Mike Morgan, who guided me through a brief foray into post-positivism that provided invaluable lessons in research and teaching. Finally, I want to thank Dr. Marcelo Diversi for countless Thursday afternoons spent walking through the Moments of qualitative inquiry, the importance of epistemology and ontology, and mentorship through my first independent work as a researcher. You have set an exceptionally high bar for me as an advisor. Thank you for telling me I could do this work.

Finally, I'm thankful for the support of friends and family. When I began my $\mathrm{PhD}$ program six years ago I never dreamed I would find friends who felt like family, people whose passion for thinking and debate matched my own. This dissertation has, somewhat ironically, threatened to strain my relational ties, and I've been sustained throughout the process by days spent writing with papers spread out on friends' carpets and nights sitting at their dining room tables, writing and re-writing while dear ones are in the next room, ready with feedback or much-needed mindless television breaks. Joseph, Sandy, Thuan, 
RELATIONAL WORLDS OF FIRST-GEN STUDENTS

Ben, Roberta, and Gita, it is your faces I see before me when I imagine Gergen's (2009) notion of the "enchantment of we" (p. 179): friends who feel like family. I'm also thankful for the patience of my son Joshua and my partner Greg while I've been writing this dissertation. Thank you for enduring my endless hours at the computer, for cooking meals for me, and providing a room of one's own.

And last but not least, I'm thankful for my friend Aaron, who was the first person to make me question what happens to young people after they leave foster care. If I had not met you I'm not sure I would have ever dreamt of research or getting a $\mathrm{PhD}$. You have changed my life. 


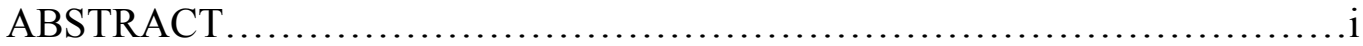

AKNOWLEDGEMENTS ............................................ii

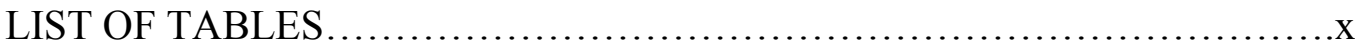

LIST OF FIGURES ...................................................

CHAPTER 1. INTRODUCTION. ........................................................

Research Questions ...............................................................

Background and Importance of the Study for Social Work .............6

CHAPTER 2. REVIEW OF RELATED LITERATURE ................................16

An introduction to first-generation students ................................16

Framing first-generation students: Academic Risk .....................22

Re-framing: Integration and relationships.................................28

Theoretical frameworks: Integration and Reproduction ................41

Summary and locating first-generation students in social work .....54

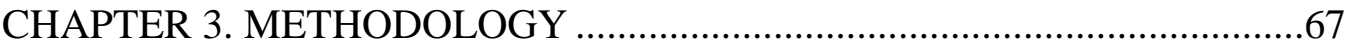

Feminist Standpoint Theory ...................................................69

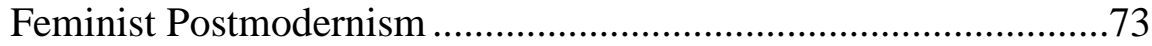

Focus group methodology ......................................................8

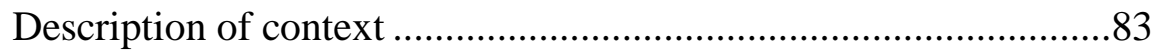

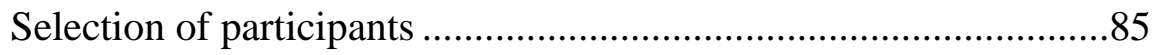

Data Collection.........................................93

Data Analysis......................................... 103

Analysis of single-group transcripts.....................104

Analysis of transcripts across groups...................111 
Writing as analysis ..................................................... 114

Establishing trustworthiness ................................................117

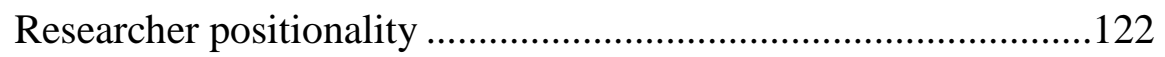

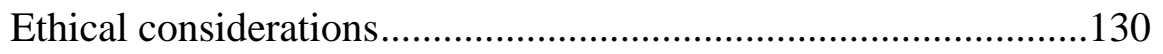

CHAPTER 4. FINDINGS ........................................... 134

How do first-generation students describe their relational worlds?

Experiences of

support............................................. 140

Pursuing school on your

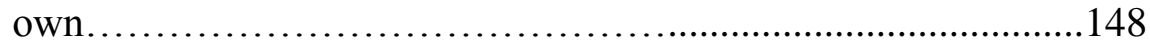

Potential for

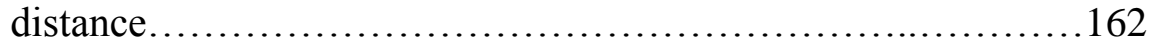

Working to stay integrated in home

cultures................................................185

Being "in-between” school and home: Borderlands (Anzaldúa,

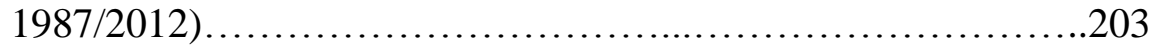

Working to integrate: Relational experiences in school.....

Implications for professional socialization

Being supported and giving support.

Elitism and valuing of different types of work.

Creating a new "future route": Family history, gender, and cultural expectations.......................................... 238

Family voices and becoming a social worker.

Field experiences: race, gender, and interruptions. 
In closing: First-generation status as a relational

experience.

Moving

up?.....

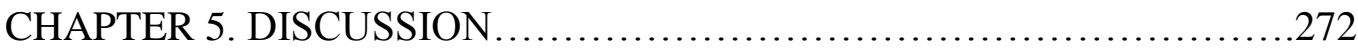

Family is an important source of support.

Performing without a net: Students are on their

own.

Conversations and integration go hand in

hand.

The work of integration: How ready are schools for first-generation students.

Limitations

Implications

References

Appendices

Appendix A. 15 Propositions derived from Tinto 324

Appendix B. Participant Consent Document 326

Appendix C. Sample Recruitment flyer. 328

Appendix D. Focus Group Interview Questions .329

Appendix E. Listening guide. ... 


\section{LIST OF TABLES}

TABLE

PAGE

$1 \quad$ Participant demographics .......................................... 88

2 Outline for analysis of transcripts across groups........................113 


\section{LIST OF FIGURES}

FIGURE

PAGE

1 Guiffrida's (2006) Recommended changes to Tinto's Theory..........50

$2 \quad$ Visual outline of primary research findings........................ 139 


\section{CHAPTER 1: INTRODUCTION}

Increasing access to higher education for underrepresented students is regarded as a benefit of the post-World War Two expansion of higher education in the United States ${ }^{1}$. It's estimated that $30 \%$ to $50 \%$ of all college students are "first-generation," (Berkner \& Choy, 2008; Strayhorn, 2006), and this proportion is projected to increase. Over the last few decades, greater attention has been paid to the needs of first-generation students, based primarily on empirical studies highlighting disparities in academic performance between first-generation students and their peers whose families have greater exposure to higher education. Studies using a cultural capital framework (Bourdieu, 1986) have confirmed that family experience with higher education does make a difference in academic (Collier \& Morgan, 2008; Padgett, Johnson, \& Pascarella, 2012; Pascarella, Pierson, Wolniak, \& Terenzini, 2004) and social (London, 1989, 1992; Lowery-Hart \& Pacheco, 2011; Stuber, 2011) integration. Traditional images of the college student as an independent actor invested solely in his (or increasingly, her) own educational, occupational, and personal development inform notions of the importance of separation from family during schooling. Indeed, "breaking away" (London, 1989) from family is one of the prominent themes in the literature describing the relational experiences of firstgeneration college students, and it is sometimes assumed or implied that separation from family is a necessary part of college success. However, the literature provides numerous examples of the deep and ongoing connections to family among first-generation college

\footnotetext{
${ }^{1}$ See Ryan and Sackrey (1996) for a historical sketch of the broadening of public institutions of higher education.
} 
students (Bradbury \& Maher, 2009; Bui, 2002; Gofen, 2009; London, 1989; Orbe, 2004;

Stieha, 2010).

The experiences of first-generation students in schools of social work are relatively unexplored, limited to a demographic study of undergraduates in a social welfare program (Hodges, 2000) and a personal narrative (Carter-Black, 2008). The absence of social work from discussions of first-generation students is noteworthy, given the demographics of first-generation students in the United States ${ }^{2}$ and social work values of social justice and self-determination (NASW, 2008). In particular, the social work value of human relationships suggests that an exploration of the relational experiences of first-generation school of social work is within the purview of social work research. These experiences also have important implications for the process of professional socialization and for recruiting and retaining a diverse group of social workers and professional helpers (Casstevens, Waites, \& Outlaw, 2012), and infusing social work values of justice and diversity in higher education (Saulnier \& Swigonski, 2006).

This study drew from focus group discussions with first-generation students in a school of social work ${ }^{3}$ centered on the conditions of their relational lives ${ }^{4}$. Because many

\footnotetext{
${ }^{2}$ Who, as Cole (2008) noted, tend to be members of groups that experience marginalization, discrimination, or oppression based on race, class, and gender, or in other words, people that social workers are likely to encounter in their daily practice.

${ }^{3}$ Not all of these students are or will become social workers. The majority of students are pursuing undergraduate and graduate level social work degrees, but students in a broader liberal arts degree, Child and Family Studies, will also be included in the proposed study.

${ }^{4}$ Exploring the conditions of relational life requires revisiting and considerable revision of the notion of individuals as bounded beings, a construction embedded in several centuries of Western thought and philosophy (Gergen, 2009). Focusing on relational lives asks one to imagine relationships not simply as bonds between otherwise independent individuals, but as the wellsprings from which our identities as independent individuals emerge. We do not develop in isolation, but rather are created by the negotiation
} 
first-generation students are unaware that being the first in their family has any meaning for their school experience (Orbe, 2004), or that others may share feelings of being a newcomer (Stuber, 2011), I used focus groups to foster connections between students and prompt deeper reflection. Relationships with family, community members, and others in the school setting were explored with attention to the importance of relationships in shaping notions of who we are, which has implications for the process of socialization into the role of a social worker or as a member of the helping professions ${ }^{5}$. In addition to questions of identity formed in relationship, issues of separation and/or connection to family and home communities were explored, as well as the ways that students navigated the social or cultural distance that is often assumed to be part of the first-generation experience (Guiffrida, 2006; London, 1992, 1996). Voice-centered relational data analysis (Brown \& Gilligan, 1992) was used to interpret the narratives that emerged from these discussions.

This dissertation is organized into five chapters. The first chapter outlines research questions and offers an introduction to the emergence of these questions from my own experiences and locates the questions within higher education research and social work. The second chapter provides a review of relevant literature, beginning with a brief introduction to first-generation students, their academic needs, and interventions.

and re-negotiation of our identity in relationship with those around us. For example, each day I enact multiple versions of myself: the stranger on the bus, a student to my dissertation committee, a "professor" to my students, a colleague, confidant, or cheerleader to my fellow doctoral students, a mother, sister, or partner when I return home. Each identity carries with it a multiplicity of possibilities, each possible version dependent on the meanings co-constructed with others. Rather than seeing identity as something created through individual thoughts and actions, attending to relational life reveals the interdependence of identity and the power of relational confluence in the ways we see ourselves and the world.

${ }^{5}$ Counselors, educators, youth workers and others are members of the helping professions. 
The bulk of the literature review is focused on what is known about the relational experiences of first-generation students: questions regarding separation from families and integration into school, both academically and socially. Various themes within the literature are outlined, including "breaking away" (London, 1989), struggles to integrate, resistance to integration, and the existence of first-generation students as border crossers ${ }^{6}$ between the worlds of school and home. Next, two prominent theories detailing processes of integration and social reproduction are described and anchored within the literature on first-generation student experiences. Finally, the literature and theories are situated within a social work perspective, and I draw from what is known about the experiences of "underrepresented" students in social work to demonstrate a need for attention to the relational experiences of first-generation students in a school of social work. The third chapter outlines the methodology, including an introduction to strands of feminisms that inform this work (feminist standpoint theory and feminist postmodernism or post structuralism), focus group methods, a description of research methods (context, selection of participants, and data collection), and an overview of voice-centered relational data analysis (Brown \& Gilligan, 1992). Finally, I include discussions of trustworthiness, my position as a researcher, and ethical considerations. The fourth chapter shares the findings, which are organized into two broad sections, each focused on one research question and broken down into smaller sections exploring the support students receive, their experiences of being on their own in schooling, the potential for

\footnotetext{
${ }^{6}$ Many students' descriptions here are reminiscent of Gloria Anzaldúa's (1987/2012) borderlands: those who move from a home culture into an educational culture which is markedly different in terms of values may work to establish a place in both worlds, and in doing so develop a unique ability to "see" both worlds. However, there also exists the possibility for exclusion in both worlds, and the need for construction of an identity in between.
} 
distance from home cultures and the work they do to maintain connections in their home cultures while simultaneously working to become connected in school, as well as the implications of these findings for the process of professional socialization. Finally, in the fifth chapter I've put these findings back into conversation with the literature on firstgeneration students that explores the importance of cultural capital (Bourdieu, 1986) and prizes social and academic integration (Tinto, 1975, 1993). Instead of assuming that integration in school is the primary goal for first-generation students, I've argued that the work students do to stay integrated in their home cultures is important and deserves attention.

\section{Research Questions}

This study explored the relational worlds of first-generation college students in a school of social work. Specifically, the focus here was on the conditions of relational life for students from families and communities who are new to the university setting and implications for the process of professional socialization. Because first-generation students do not always share a sense of group identity, focus groups were used as a means of data collection that highlighted interaction and relationships, and may have deepened participants' reflections on their own experiences. Voice-centered relational data analysis (Brown \& Gilligan, 1992) was used to interpret student stories in response to these research questions:

1) How do first-generation students in a school of social work describe their relational worlds? How do they describe relationships with family, community members, and within the school setting, and (how) do they experience issues of 
RELATIONAL WORLDS OF FIRST-GEN STUDENTS

separation and/or navigate cultural or social distance between home communities and school?

2) What implications do these relationships have for the process of professional socialization for first-generation students? (How) do relational experiences shape constructions of identity as a social worker and/or professional helper?

\section{Background and Importance of the Study for Social Work}

After returning to school to complete a bachelor's degree nine years ago, I became interested in access to higher education for other young people who were potential first-generation college students: teens I worked with in an arts-based summer program, current and former foster youth in a community transition program, and the young (and not-so-young) people in my own family. Together we attended workshops and outreach events organized by school districts, the local university, and the state higher education coordinating board. Even though I was currently riding on the high of being able to go back to school, I found myself profoundly unsettled by aspects of these events. While attending a workshop on my university campus with a group of middle school students, I felt my excitement dissipate during the morning "welcome," witnessing the clear sense of discomfort in the speaker's face and her halting speech as she addressed the group of young people, many of them people of color, and most from lowincome families. A few months later, at an outreach event designed for students and their families to sign up for a statewide scholarship, I stood in a darkened cafeteria on a college campus that has always felt inviting to the community while low-income families of all colors, languages, and nationalities were enticed by the iconic bass line from the 
O’Jays "For the love of Money" (Gamble, Huff, \& Jackson, 1973) while being showered with fake green dollar bills. I pondered my own degrees and the lack of a connection to a higher income, and wondered if we were selling these students and families an inaccurate picture of the results of college. I felt sensitive to the tensions that such an inyour-face approach might elicit for people for whom money can be an every day, even every minute, concern. I also couldn't help noticing the unease so many of the presenters welcomed these "underrepresented" students and families to the campuses; students were invited to join the "club" at college, but whose space was it, really?

Despite my own discomfort with the emphasis on college as a means of getting rich, I could not deny the connections between educational attainment and economic security and social mobility. In 2009 the annual earnings gap between young men with a bachelor's degree and young men with a high school diploma was $\$ 20,000$ (NCES, 2011). For women the same gap was $\$ 18,100$. Over a lifetime, this earnings difference is estimated to be as high as $\$ 1,000,000$ (Day \& Neuberger, 2002). It follows, then, that completion of a college degree exerts a powerful influence on social mobility. Haveman and Smeeding (2006) argued that most Americans endorse higher education as a meritocratic means for providing avenues for social mobility, although it is increasingly less likely to fulfill this role ${ }^{7}$. Despite the increased difficulties students and families face

\footnotetext{
${ }^{7}$ Several factors, including the role of finances in academic enrichment and college preparation, support in the college application process, a lack of knowledge about college, particularly cost and the availability of financial support, the spike in college costs, and decline in needs-based financial aid and a shift towards merit-based financial aid, have played into this trend, according to Haveman and Smeeding (2006). For another view of the ways that colleges have furthered stratification by race and income amidst increasing access, see Carnevale and Strohl (2010).
} 
in obtaining a college degree amidst declining federal and state support, many still agree that higher education is necessary for ensuring economic stability.

Perhaps one of the most compelling and overlooked reasons for promoting access to higher education is the strong relationship between educational attainment and physical health. Mirowsky and Ross (2005) noted that the impacts of education on health throughout the lifespan can be measured on socioeconomic, behavioral, physiological, and intracellular levels. Using data that measured levels of educational attainment and physical impairment throughout adulthood (ages 20 to 90), these authors demonstrated that people without a high school diploma reported levels of physical impairment equal to those of college educated persons who were 20 to 30 years older. For high school graduates, the difference was 15 to 20 years. At all ages, levels of physical impairment increased at faster rates for those with lower levels of education.

Educational attainment, then, can be posed as a means of ensuring economic stability, social mobility, and physical health. But there are other, less obvious goals I brought to this work that require some clarification. First, I don't embrace the position that college is necessary for everyone, or even desired by everyone. Vocational training and apprenticeship are viable pathways for supporting employment and career development. The second point is subtler, though, and somewhat at odds with the previous statement. Ideally, a college education is not limited to vocational preparation, but promotes deeper engagement with learning (Tugend, 2012). An emphasis on learning is easily obscured in the increasingly frequent questions about the value of a college degree (Abel \& Deitz, 2014; French, 2010; Kapur, 2013). Even interventions which 
focus on the laudable goal of education as a means of reducing poverty (Deming \& Dynarski, 2009; Richburg-Hayes, 2008) may overlook gains from college which aren't as easily calculated in cost-benefit analyses. As an educator and someone whose life has been irrevocably changed by higher education, I put forth the following proposal as an effort to support education as a valuable end on its own. As Howard London (1996) writes, "if our rhetoric about democratizing higher education is to have any integrity...we need to avoid the trap of knowing the cost of everything and the value of nothing" (p. 13, emphasis added).

The argument I pose for paying attention to the experiences of first-generation social work students is about more than education as a means of employment, although I realize that for many students, getting a job is the primary goal. My argument here is about access to education, and rooted in the principles of critical pedagog $\mathrm{y}^{8}$; this study was focused primarily on the value placed in knowing the learner and their context (Freire, 2005). While first-generation college students have, by definition, been successful in gaining access to college, a word about access and the role parental education plays in determining who goes to college is in order. Choy (2001) documented the power of parental education in her analysis of longitudinal data from the National Educational Longitudinal Study of 1988 Eighth graders: among students who completed high school in 1992, 92.5\% of students whose parents held a bachelor's degree were enrolled in college by 1994. For students whose parents had some college, $74.7 \%$ had

\footnotetext{
${ }^{8}$ Critical pedagogy is an approach within education, cultural studies and related fields which is beyond the scope of this proposal, but involves issues in education related to cultural capital, dialogue, the "hidden curriculum" of classrooms, discourse, and experiences of marginalization and oppression. For a good primer, see Wink (2005).
} 
enrolled by 1994, and for students whose parents had a high school education or less, $59 \%$ had enrolled by 1994. Perhaps even more striking were the differences in proportions of students who enrolled in four-year colleges and public two-year colleges. Students whose parents had a high school degree were about as likely to be in enrolled in a four-year $(26.9 \%)$ or public two-year $(27.3 \%)$ school. Students whose parents had attended some college were more likely to be enrolled in a four year (41.6\%) than a public two-year (29.5\%) school, and students who had at least one parent with a bachelor's degree were overwhelmingly enrolled in four-year institutions $(70.8 \%$, versus $18 \%$ in public two-year institutions). Choy (2001) used Berkner and Chavez's (1997, as cited in Choy) outline for college preparation to demonstrate how parental education played a role in supporting students at each step of the process. While most (93\%) students surveyed in eighth grade expected to continue their education beyond high school, by tenth grade differences emerged in preparation that were dictated by parents' education levels. Students whose parents held bachelor's degrees were more likely to be at least minimally academically prepared for college coursework during their secondary education (79\% compared to 53\%), were more likely to take SAT/ACT tests $(78 \%$ compared to $49 \%$ ), and were more likely to receive parental help (but about equally likely to receive help at school) in completing college applications. Throughout the process, parental educational attainment functioned as an important predictor of access to higher education.

Therefore it may be difficult for college administrators or for first-generation students themselves to imagine issues of first-generation students in terms of access. By 
some definitions, these are already students who have beaten the odds in terms of achieving "access" to higher education. This study explored issues of access not as a one-time event that is fulfilled upon enrollment, but as an ongoing process that, for some students, involves the negotiation and re-negotiation of their relationships within family and community and school. Much has been written about the academic struggles of firstgeneration students, but London (1996) has aptly pointed out that for many firstgeneration students, college completion occurs without significant difficulties. However, for some first-generation students, the experience of earning a degree is incredibly disruptive to their families and sense of self (London, 1996). Literature on the experiences of first-generation students indicates that students respond to the process of education in varying ways: "breaking away" from family relationships to integrate, feeling a sense of ongoing marginalization in college, rejecting and resisting integration in college, and existing as border crossers or "heroes" to family members who hope to follow in their footsteps. However, aside from a demographic study of undergraduates in a social welfare program (Hodges, 2000), very little is known about first-generation students in social work. These negotiations and re-negotiations of identity and relationships have important implications in social work and other closely related fields where a large part of the professional socialization process involves identifying with the goals and values of a larger group, as well as attending to one's identity as a professional helper (Barretti, 2004; Wiles, 2013).

Traditionally, the needs of first-generation college students have been documented by sociologists and education researchers; social work has not been active in 
the discussion (P. Collier, personal communication, Nov. 21, 2013). Cole's (2008) narrative analysis of the educational stories of low-income first-generation college students provides one of the few examples of research in social work documenting the needs of first-generation college students. Attention to the needs of first-generation college students is important to social work for at least two reasons: because of who firstgeneration college students are and because of the values held by social work as a profession. Social work's values of social justice, the dignity and worth of the person, and the value of human relationships suggest that the needs of first-generation students are within the scope of social work and that social workers may be equipped to address those needs (NASW, 2008).

Social work's value of social justice asks social workers to focus on social change, particularly when that change addresses the needs of members of groups who face oppression, discrimination, and marginalization (NASW, 2008). Demographic patterns in the group of people who are first-generation college students indicate the need for attention to the ways that racism, classism, and sexism structure opportunities for educational attainment. First-generation students are more likely to be people of color (Bui, 2002; Chen, 2005; Terenzini, Springer, Yaeger, Pascarella, \& Nora., 1996), and/or come from low-income families (Bui, 2002; Chen, 2005; Terenzini et al., 1996). Firstgeneration students are more likely to be women, with proportions of women in samples ranging from a low of 55\% (Bui, 2002) to a high of 71\% (Padgett et al., 2012). As Cole (2008) noted, first-generation students are often members of groups that social workers are likely to interact with in their day-to-day work, and social workers are uniquely suited 
to address educational barriers related to both historical and contemporary experiences of racism, classism, and sexism. Furthermore, the value of social justice compels social workers to consider the needs of first-generation college students as members of groups who have traditionally been excluded from higher education. Social change focused on addressing issues of poverty and unemployment is central to the value of social justice, and the links between educational attainment and economic need are clear. Supporting first-generation college students in the pursuit of higher education is the work of social work.

Second, social work's value of the dignity and worth of the person suggests that the needs of first-generation college students are important to social work. Central to this value is the responsibility for supporting the self-determination of individuals as well as a responsibility to larger society (NASW, 2008). Educational attainment, particularly the completion of a bachelor's degree, is one of the most reliable methods of ensuring economic independence and a level of self-determination. But research detailing the experiences of first-generation college students suggests that students are not the sole beneficiaries of their college education; education is sought as a means of creating a path for others to follow (Gofen, 2009), preparing to financially support family, or bringing families honor (Bui, 2002; Orbe, 2004). Several studies describe the centrality of family relationships to first-generation college students (Bradbury \& Maher, 2009; Bui, 2002; Gofen, 2009; Stieha, 2010); “carrying" family was a common theme among students in Orbe's (2004) focus groups. A handful of studies have explicitly focused on the relational worlds of first-generation college students (e.g., London, 1989; Lowery-Hart \& 
Pacheco, 2011; Orbe, 2004), but first-generation students are almost completely absent in the social work literature. Social work recognizes the importance of relationships in strengthening individuals, families, organizations, and communities, and as such is wellpositioned to increase understanding of the relationships that support first-generation college students, and may in turn support others as well.

Although studies of first-generation students are extremely limited in social work, there are some indicators that social work education should pay attention to the experiences of first-generation and other underrepresented students. Carter-Black (2008) penned a first-person narrative of her own experiences as a first-generation student and difficulties posed by both her family's unfamiliarity with college and experiences of racism in the classroom during the post-Civil Rights push to include students of color in primarily white institutions. At least one generation later, Davis (2004) shared her own reflections of experiencing racism in an MSW classroom. Daniel (2007) relayed the stories of social work students of color who echoed experiences of racism in classroom and field placements that went unaddressed, racial and cultural isolation, and pressures to ignore racism as part of the process of becoming a social worker. While based on small samples, and in two cases representing only one person's perspective, these experiences are important to attend to in social work education, based on the social work value of social justice ${ }^{9}$. Even less is known about the experiences of low-income students in schools of social work. Saulnier and Swigonski (2006) pointed to the importance in social work, particularly feminist social work, of transforming institutions that have

\footnotetext{
${ }^{9}$ And this issue is clearly not limited to social work education. See for example Institutional Racism and the Social Work Profession: A call to action (National Association of Social Workers, 2007).
} 
traditionally excluded some, and building institutions that reflect the social work values of diversity. Turning our attention to experiences of first-generation students in a school of social work is an important first step.

Renn and Reason (2013) highlighted a final, often overlooked reason for attending to the needs of first-generation students:

...Perhaps the mythical "American college student" - we will call him John -still exists: a full-time student who came directly to college from high school, John lives in the residence halls, works on campus ten hours a week, and takes a full course load that has him on track to graduate in four years with a bachelor's degree from a selective public university. John is white, Christian, heterosexual, middle class, and without disabilities... (ix-x).

As Renn and Reason (2013) noted, the majority of college students today are not "John" (or even "Jane," his female counterpart). However, "John" is often still the assumed model for college going in educational interventions and among student affairs personnel. First-generation students are part of a larger group of non-traditional students who, for many reasons, depart from these traditional conceptions of college students, and their experiences deserve attention in social work. 


\section{CHAPTER 2: REVIEW OF RELATED LITERATURE}

In this review, I've drawn from the growing literature on first-generation college students to describe what's known about their experiences in higher education. Because the issues faced by first-generation students have not been widely discussed in the social work literature, I've begun with an introduction to terminology and demographics, as well as what's known about broad differences between first-generation and traditional college students. Because so much of the literature on first-generation college students is focused on their academic performance, a brief overview is included. However, because the focus of this research is on the relational worlds of first-generation students, I've included a longer discussion of what's known about their relationships inside and outside of school. Next, I've outlined two theories commonly used in the literature on firstgeneration students that have important implications for intervention. One theory examines integration into the academic and social spheres of college, and another explores the backgrounds students bring to college and the cultural tools they possess, along with the cultural ideals, attitudes, and experiences valued by the institutions of higher education (although this is explored to a lesser degree in the literature). Finally, I've attempted to connect broad themes in the literature on first-generation students and their relationships to observations of the experiences of students from "underrepresented" groups in schools of social work and explore the implications for the process of professionalization among first-generation students in a school of social work.

\section{An Introduction to First-Generation Students}


RELATIONAL WORLDS OF FIRST-GEN STUDENTS

Researchers differ in their definitions of "first-generation" college students. The most conservative definitions include only students for whom neither parent has any postsecondary education experience (Bui, 2002; Chen, 2005; Padgett et al., 2012; Pascarella et al., 2004; Terenzini, Springer, Yaeger, Pascarella, \& Nora, 1996), perhaps due to the finding that some educational advantages accrue to students whose parents have some college experience (see for example Padgett et al., 2012). More liberal definitions include all students for whom no parent has a bachelor's degree (Choy, 2001; Collier \& Morgan, 2008; Stuber, 2011). In order to be as inclusive as possible in considering literature on first-generation college students, and in recognition of the finding that having at least one parent with a bachelor's degree is a strong predictor of access to and performance in postsecondary education (Choy, 2001; Pascarella et al., 2004), I have chosen to use the more liberal definition of "first-generation" college student when outlining eligibility requirements for this study. This included students whose parents had varying levels of postsecondary educational experience (no education beyond high school, some college without degree completion, completion of a transfer, technical, or vocational degree), but have not completed a bachelor's degree. By using this broader definition, I allowed for the inclusion of students whose parent(s) may have made it into college, but found their experiences there too untenable to remain. More conservative definitions would define these families as having college experience, but it is arguable whether a parent's negative experiences of college have a positive benefit for students. 
Research on first-generation students falls into several broad categories outlining access, college choice, and the precollege characteristics of first-generation students (Bui, 2002; Chen, 2005; Reid \& Moore, 2008; Terenzini et al., 1996); academic performance and college experiences (Bradbury \& Maher, 2009; Bui, 2002; Chen, 2005; Collier \& Morgan, 2008; Padgett et al., 2012; Pascarella et al., 2004; Reid \& Moore, 2008; Stuber, 2011; Terenzini et al., 1996), and persistence, or completion of a degree (Chen, 2005; Choy, 2001). Note that several studies cover multiple aspects of the first-generation experience and that the literature tends to follow a general temporal outline. Students must first gain access to postsecondary education (which involves planning to attend, college-preparatory coursework in high school, taking admissions tests (most commonly the ACT/SAT), applying and enrolling (Berkner \& Chavez, 1997, as cited in Choy, 2001)). Upon enrollment, these students may (or may not) experience some of the struggles associated with being a first-generation student. And finally, these students' experiences are documented through the rates of bachelor's degree completion. While gaining access and the completion of a degree are important aspects of the firstgeneration experience, my focus in this work is on the relational experiences of firstgeneration students. Some of the work outlining issues of access has been included in the introduction (see discussion of Choy, 2001) to demonstrate the power of parental education in predicting access to postsecondary education, and some of the literature on precollege characteristics will be drawn from to provide a demographic picture of firstgeneration college students. Likewise, while measures of persistence and completion are important, my focus is on the conditions of relational life for first-generation students (indeed, one can likely argue that relational experiences have a bearing on rates of 
RELATIONAL WORLDS OF FIRST-GEN STUDENTS

retention and completion - when schooling is too disruptive to one's relationships, it may be impossible to remain).

\section{Demographics of First-Generation Students}

While not all campuses track first-generation status among their students, the proportion of first-generation college students is estimated at approximately $30 \%$ nationally, and growing (Strayhorn, 2006). Others estimate the proportion of firstgeneration college students to be as much as one-half of all students enrolled in college (Berkner \& Choy, 2008). While the group of students who fall under the umbrella of "first-generation" is so large that it is difficult to paint a definitive picture, firstgeneration students tend to differ from "traditional" or "continuing generation" (Stephens, Hamedani, \& Destin, 2014) college students in their racial or ethnic backgrounds, their socioeconomic or class background, and gender.

First-generation students are more likely to be people of color, with AfricanAmerican and Hispanic students overrepresented in some samples of first-generation students (Chen, 2005; Terenzini et al., 1996) and Asian and Latino students overrepresented in others (Bui, 2002). Other researchers have made fewer distinctions in measuring race or ethnicity, for example, categorizing students as either "white" or "people of color," and instead focusing their lens on specific levels of parental educational attainment (parents have no college experience, parents have some college, one parent has a bachelor's, both parents have a bachelor's or above...) (Padgett et al., 2012). In this study the proportion of students who were white increased steadily with each level of parental education, with white students making up 57\% of the first- 
generation group and $88 \%$ of the group whose parents both have a bachelor's degree or above.

Family income is also a factor in determining intergenerational access to postsecondary education. Several studies noted that first-generation students are more likely to come from low-income families (Bui, 2002; Chen, 2005; Terenzini et al., 1996). Chen (2005) found that first-generation students were about twice as likely to report a family income of $\$ 25,000$ or less (in 1991) as their peers from families who had some postsecondary education $(50.3 \%$ of first-generation students compared to $25.9 \%$ of students whose parents had some college). A more recent sample of full-time, first year undergraduates reflected similar differences in income levels by parental education, with the lowest mean parental incomes among first-generation students $(\$ 49,170)$ and progressively higher incomes among students whose parents had higher levels of education (parents had some college $(\$ 61,999)$, parents had one bachelor's degree $(\$ 77,614)$, both parents with a bachelor's degree $(\$ 108,994)$, and at least one parent held a master's degree $(\$ 125,662)$ (Padgett et al., 2012). The majority of first-generation students in Bui's (2002) sample identified as "poor" (14.06\%) or "working class" $(39.06 \%)$. In contrast, the students in Bui's sample who identified as "poor" or "working class" were less likely to come from families with parents with some college (20\%) or a bachelor's degree (5.88\%).

Regardless of parental education levels, women are more likely to be enrolled in college than men, but women tend to be over-represented among first-generation college students, with the proportions of women in samples of first-generation students ranging 
from a low of 55\% (Bui, 2002) to 71\% (Padgett et al., 2012). The proportion of women in Padgett and colleagues' sample decreased as levels of parental education increased. Women composed $71 \%$ of the first-generation and "parents have some college" groups, $66 \%$ of the "one parent has bachelor's" group, $65 \%$ of the "both parents have bachelor's or above" group, and $62 \%$ of the "one parent has master's or above" group. A similarly gendered pattern was found in Chen's (2005) longitudinal examination of student transcripts, with higher proportions of females among first-generation students (60.2\%) than among students whose parents had some college (54.6\%) or students whose parents had a bachelor's degree or higher (48.5\%). Terenzini and colleagues (1996) noted the higher proportion of women among first-generation students in their sample (61\%) than in their sample of traditional students (53\% were women). Because of deeply embedded assumptions about family responsibilities and care work in contemporary United States society (Coltrane, 2000; Lincoln, 2008), it comes as little surprise that first-generation students (many of whom are women) were more likely to report responsibilities for caring for dependents. However, Terenzini and colleagues (1996) offered the only largescale study to inquire into childrearing responsibilities, noting that first-generation students have a higher average number of dependent children than their traditional student peers. Because responsibilities to family and connections to family are an important part of this research, I will return to a broader discussion of family relationships among first-generation college students below.

Little is known about first-generation college students in social work programs. John Hodges (2000) conducted a survey of undergraduate students enrolled in a social 
welfare program at a highly selective four-year university. Among a sample $(N=102)$ of undergraduate social welfare majors, first-generation students $(n=42)$ were found to be slightly older (25.5 years compared to 23 years) than their peers, and more likely to be Hispanic or Latino/a. There was a larger proportion of males among the first-generation students $(21.4 \%$ compared to $6.7 \%)$, but again women comprised the overwhelming majority (78.6\%). Similar to wider demographic patterns in the wider body of literature describing first-generation college students, first-generation college students in the social welfare program were less likely to be Caucasian or Asian. However, unlike trends in the larger body of literature, there were a slightly smaller proportion of African-Americans among first-generation students. Hodges questioned whether the smaller proportion of African-American students among first-generation students in the sample might be due to broader demographic patterns in the region, with higher proportions of Hispanic and Latino students, and speculated that perhaps African-American students on that campus might be less likely to select social welfare as an undergraduate major, but did not suggest possible explanations. First-generation students in this study were also more likely to be children of parents who weren't U.S. citizens and to have learned another language before learning to speak English (Hodges, 2000).

\section{Framing First-Generation Students: "Academic Risk"}

It is difficult to engage with the literature on first-generation students without seeing their needs as being dominated by academic challenges. The literature is dominated by descriptions of the struggles of these students in comparison to their peers. In Chen's (2005) study of first-generation students who had graduated from high school 
in 1992 and enrolled in college by 2000, first-generation students were just as likely to persist in school (defined as "either attaining a degree or still enrolled"), but on average had completed about half of the postsecondary credits (66) of traditional college students (112). Even though first-generation students tended to take fewer classes and complete fewer credits, they also tended to earn lower grades than traditional students in those classes. Following their first year in college, first-generation students had lower GPAs (2.5) than their peers (2.8), and had earned fewer credits (18 compared to 25) (Chen, 2005).

Studies that focus on the cognitive and psychological impacts of college among students in diverse institutions (four-year research universities, regional universities, liberal arts colleges (public and private), historically black colleges, and two-year community colleges) paint a more nuanced picture. After controlling for differences (demographics, high school experiences, degree plans), Terenzini and colleagues (1996) found that first-generation students made similar gains in mathematics and critical thinking, but did not make as much progress as their peers in reading comprehension. Similarly, in follow-up assessments of cognitive and psychosocial changes following the first year in college, Padgett and colleagues (2012) found that first-generation students were similar to their peers in critical thinking, higher order moral reasoning, and their need for stimulating cognitive activities, but different in their attitudes toward literacy (enjoyment of reading, especially poetry, scientific, and historical texts). First-generation students also showed lower levels of intercultural effectiveness and openness to diversity, and psychological well-being. Pascarella, Pierson, Wolniak, and Terenzini (2004) 
followed students in their second and third years of college in 18 four-year institutions and found that first-generation students had lower cumulative grade points averages through their third year of college and completed significantly fewer credits. Differences were particularly strong between first-generation students and those whose parents were highly educated (more than one parent had graduated from college). However, the picture of first-generation students again emerged as more complicated than simply one of academic deficits. First-generation students were not any different from their peers in writing, critical thinking, or openness to diversity and challenge. And first-generation students were more likely to endorse an internal locus of attribution for success, that is, to look to their own efforts and abilities as explanations for academic progress. Firstgeneration students also showed stronger preferences for higher-order cognitive tasks and seemed to benefit more from engagement in the classroom and academic challenges: numbers of hours spent studying, number of term papers written, and the number of unassigned books read had stronger impacts on first-generation students' critical thinking, writing, openness to diversity, perception of control and degree plans than they did for traditional students.

In Cole's (2008) narrative analysis of the educational stories of low-income firstgeneration college students, academic challenges were prominent among stories of students who left college. Students attributed their academic challenges to pre-college ability, balancing the many demands on their time, their inability to meet the standards of their chosen majors, and lacking strategies to improve academic performance. Distress related to grade performance was an underlying feature of all of the stories of students 
who had faced academic challenges. Students described feeling anxious and uncertain about their future, feelings of being unprepared for academic work in a university, and the strains of balancing paid work with school work.

First-generation students also tended to differ from traditional students in the way they attend higher education, that is, their enrollment patterns were markedly different from students who came from families where at least one parent had a bachelor's degree. First-generation students tended to begin college at an older age, were more likely to begin their education at a two-year institution, more likely to attend school part-time, and to disrupt enrollment, including starting and stopping, returning to school, and enrolling for short periods (less than one year) (Chen, 2005). It is likely that enrollment patterns are largely influenced by the common finding that first-generation students were often employed more hours than traditional students and many live off campus (Bradbury \& Maher, 2009; Orbe, 2004; Pascarella et al., 2004; Terenzini et al., 1996). Higher levels of employment among first-generation college students and higher levels of socioeconomic stress may also affect priorities students attach to competing demands. Choy (2001) noted that among all postsecondary students that were employed, first-generation college students were much more likely to identify as an "employee enrolled in school" (38.3\%) than their peers whose parents had a bachelor's degree $(17.6 \%)$, who were more likely to identify as a "student working to meet expenses."

\section{Interventions Tailored for First-Generation Students}

Noting the difficulties first-generation college students face, various interventions have been implemented to address retention among first-generation students. As first- 
generation students shared in a report by Engle and colleagues (2006), it wasn't so much getting in to college that was difficult, but remaining in college once enrolled. These students found bridge programs that helped them prepare for the demands of college helpful. In a difference-education intervention, first-generation and continuing generation students attended an orientation at the beginning of the academic year in which upperclassmen shared their experiences of college and gave advice in a panel format that took two forms: in one panel students offered advice and insights and in a second panel the same students offered advice and insights while also discussing their social class background and specifying their generational status (first-generation or continuing generation) (Stephens et al., 2014). At the end of their first-year, firstgeneration students who attended the panel discussion where social class differences and generation status were highlighted showed greater tendencies to access resources (e.g. talk to faculty regarding learning issues), higher grade point averages, and scored better on assessments of psychosocial health. The authors emphasized that rather than downplaying difference, highlighting differences and attending to identity seemed to support both first-generation students and continuing generation students, who also showed increases in psychological health and engagement.

Living-learning programs, which provide some academic support alongside a residential component, showed low to moderate results in fostering social and academic integration into college for first-generation college students, in comparison to firstgeneration students living in traditional residence halls (Inkelas, Daver, Vogt, \& Leonard, 2006). While not directly focused on describing an intervention, Lowery-Hart and 
Pacheco (2011) conducted focus groups with students in a campus-based "University Success Academic program" specifically targeting first-generation college students which offered tutoring and support. Likewise, Gray (2013) described a conditional program designed for students designated "at-risk" upon enrollment in Eastern Michigan University: Promoting Academic Survival and Success (PASS). Most of these students were people of color, working class, and first-generation, and the program provided academic advising, block scheduling, and participation in a class designed to support students' integration to college, UNIV 101.

The federal government has undertaken initiatives to address the needs of firstgeneration and other underrepresented students in higher education through a collection of programs which fall under the larger umbrella of TRIO (Upward Bound, Educational Talent Search, Student Support Services, the Ronald E. McNair Post-Baccalaureate Program, Educational Opportunity Centers, and Gaining Early Awareness and Readiness for Undergraduate Programs (GEAR-UP) (Jean, 2011). Beard (2006) demonstrated the impact of a TRIO program on degree completion (technical, associates, or bachelor's) among first-generation African-American students. These impacts were not seen for Hispanic students, or for older (24 years old or above) African American students. While Jean (2011) cited evidence from the U.S. Department of Education statistics that demonstrate that TRIO participants are three times more likely to complete a bachelor's degree than students who are only receiving a Pell grant, federal TRIO programs are not fully funded and currently serve approximately $10 \%$ of the eligible population. Engle and colleagues (2006) also pointed out that while these programs are helpful, they are not 
available on all campuses and students were often unaware of the presence of these programs when they did exist on their campus.

Intervention is not without its drawbacks, however. As Gray (2013) argued, students in the PASS program were framed as "at-risk," a label that emphasized students" assumed deficits. Training for instructors preparing to teach UNIV 101 highlighted the limitations of PASS students, the lack of knowledge students brought to college, and assumptions that these students came from families that didn't value higher education. Policing students was recommended, and expectations for their success were low. From some students' perspectives, support programs seemed to reaffirm their marginal status and confirm fears that they weren't able to succeed (Gray, 2013; Lowery-Hart \& Pacheco, 2011). First-generation students in focus groups also shared that the type of asking for help that these programs required of them was incongruent with their own family background and values (Lowery-Hart \& Pacheco, 2011). Interventions for firstgeneration students may risk reinforcing stigmas of educational deficits and confirming students' status as outsiders. Because the academic "risks" facing first-generation students are such a prominent theme in the literature and interventions are designed accordingly, the remainder of this review will focus on shifting the framing from one of academic (under)performance to one in which first-generation students are members of multiple relational worlds.

\section{Re-Framing First-Generation Students: Relationships and Integration}

The stereotypical "American college student" discussed in the introduction is often constructed as an individual primarily invested in their own education, career 
enhancement, and developing their own sense of self. While college is assumed to be a time of developing peer networks and establishing friendships, the stereotypical traditional college student is predominantly autonomous ${ }^{10}$. "First-generation" status, on the other hand, is inherently relational and suggests family experience in higher education is useful in preparing students to "do college" once enrolled. Explorations of the integration of first-generation students often involve some discussion of relationships.

However, before proceeding it is important to note the complexities in how students perceive their first-generation status, or even whether they feel it means anything at all. Some students reported that they think about what it means to be a first-generation college student often (London, 1989; Orbe, 2004), while others reported they being the first in their family to attend college didn't impact their experiences of integration (Stuber, 2011). Many first-generation students don't recognize themselves as part of a larger group of students who are new to college campuses (London, 1996) and many participants in Orbe's (2004) focus groups were not aware they were "first-generation" or that this might be meaningful until they were informed about the study and realized they met the eligibility criteria.

Context seemed to make a difference in how much importance students attached to their identity as "first-generation" students; students at more selective colleges were more likely to be aware of the ways they were different from their peers than students at

\footnotetext{
${ }^{10}$ Traditional ideas about college as a time of developing independence have been challenged by the recent rise in "helicopter parents" (Lum, 2006; Shoup, Gonyea, \& Kuh, 2009). However, because the focus of much parental intervention is focused on the individual student's success, I would argue the "helicoptering" does not fundamentally disrupt assumptions of college students as invested first and foremost in their own educational, occupational, and personal development.
} 
less-selective colleges, who tended to be surrounded by peers from similar backgrounds (Bradbury \& Maher, 2009; Orbe, 2004). Race, class, age, and gender also played a role in which students felt first-generation status was more meaningful: white students and students from families of middle to higher socioeconomic status (SES) who were the same age as traditional college students were more likely to say that their first-generation status didn't matter (Orbe, 2004; Stuber, 2011). Non-traditional students, women, people of color, and students from lower SES backgrounds were all more likely to identify firstgeneration status as an important part of their identity at college. The variability of the importance that students attached to their first-generation status may be the reason why the majority of students Orbe (2004) spoke with did not feel a sense of community with other first-generation students.

While students seemed to differ broadly in how salient they felt their "firstgeneration" identity was on campus, for most first-generation students, their identity as a student was always central in relationships with family. The literature about firstgeneration college students consistently demonstrates the strength of connections to family among first-generation college students (Bradbury \& Maher, 2009; Bui, 2002; Gofen, 2009; London, 1989; Orbe, 2004; Stieha, 2010). Cole (2008) noted that "family" was the one of the most-frequently mentioned topics among her study of first-generation students on an urban campus. There is disagreement as to whether these ties to family represent a hindrance to first-generation students or provide the support to help students remain in an unfamiliar environment.

\section{"Breaking away" (London, 1989): Separation from Family}


One of the most prominent notions about first-generation students flows from ideas about a presumed desire for social mobility and the assumption that first-generation students hail from families which have not been able to prepare them for college, and suggests that students must separate from families in the process of integration into college. Cited over 200 times, London's (1989) piece detailing the experiences of firstgeneration students who felt the need to "break away" from families of origin has had a powerful influence on conversations about first-generation students. London presented case studies examining patterns of change in family dynamics as students entered college. "Breaking away" from parents looked different for each student, but family relationships were always central to students' struggles to integrate in college, and more often than not, integration involved some separation from family. Some students described being both bound to former family roles and being sent out as delegates to fulfill their (or their parents') educational aspirations; these students struggled to reconcile conflicting messages from their families. A second pattern involved students being sent out as delegates, often to meet parents' unmet educational aspirations. For these students, separation to attend higher education was seen not as abandonment, but as an act of loyalty to the family. However, some of these students felt the role of delegate so intensely that they questioned whose goals they were meeting through education: their own or those of their parents? Because parents' emotional investment in their education was so heavy, these students walked a fine line between losing themselves in their parents' wishes and risking possible rejection should they chose to pursue their own goals for schooling. 
A third pattern London (1989) described included exclusion of the student by family in addition to being sent out as a delegate. For the students described, being a first-generation college student was often another stage in a lifelong pattern of feeling “different." London emphasized that family "voices" were powerful for all students, regardless of the patterns of "breaking away." He also noted that these processes were not entirely unique to first-generation college students, but were more pronounced than they may be for students whose parents also had college experiences. That is, struggles may ensue over college selection, choice of major, or grades for any college student and their family, but the core question of whether or not to attend college is unlikely to surface in a family with experience in higher education.

Other research has confirmed students' experiences of feeling separated or distanced from family through their enrollment in school, and the attendant demands on time, new learning, and perceived intellectual abilities. Appearing too smart or challenging family members' ideas was a threat to family relationships for some students (Orbe, 2004). Some first-generation students in Orbe's (2004) study described the need to downplay their identity as a student around family members who felt they spent too much time studying, challenged family ways of knowing, or had new priorities related to schooling which precluded prior relationships. Gender was often central to tensions over identity as women tried to continue to fulfill traditional family roles or exhibit culturally prescribed behaviors. Appalachian students in Bradbury and Maher's (2009) study described ending friendships that they perceived as a barrier to finishing school or a negative influence on their general health or life. These students described feeling 
"different" upon returning home and no longer enjoyed the same activities they used to pursue with friends at home.

Writing as a scholar, Laura Rendon (1992) reflected on her experiences as a firstgeneration "Mexican-American scholarship girl" and the negotiations between her family's expectations for her life and her desire to attend college. Resisting the pressures to adapt her culture, language, and class background to fit in a predominantly white institution, Rendon wrote "my parents did not understand what education could offer (or even take away)" (p. 58). Similarly, some first-generation students of color interviewed by Richardson and Skinner (1992) shared that family members told them going to college was a waste of time. These students may have persisted due to their reported desires for different types of work or different levels of social status than their family members. Cole's (2008) interviews with first-generation students highlighted separation from family as a feature of many students' educational stories. Some students were encouraged by family to seek education as a means of escaping blue-collar work that was repetitive or demeaning, while other students saw higher education as a way out of their families and their best chance at survival.

London (1989, 1992, 1996), while recognizing that not all first-generation students undergo significant transformation, focused on the social distances many students find themselves navigating upon entry into college. London suggested that college is often a point of departure for these students (a suggestion that implies individuation and autonomy are important goals for a student) and wrote “...every student making such a transition whom I have interviewed during the past several years 
has reported having to renegotiate relations with family members, friends, and, in a fundamental sense, with themselves" (London, 1992, p. 6).

\section{Complicating Integration: “Integrated, Marginal, and Resilient” (Stuber, 2011)}

While her focus was not specifically on relationships, Jenny Stuber (2011) studied integration among white, working-class first-generation students in a large public university and small private college campus. Complex pictures of integration emerged, which did not always involve struggle against or with family backgrounds. About onequarter of the students she spoke with described struggles with academic and social integration; despite remaining enrolled, these students reported persistent feelings of alienation and marginalization in college. Whether due to economic demands (such as working up to 35 hours per week or living at home to save money and commuting to campus), or the struggles required to adjust to the culture of the college campus (e.g. the Greek system and largely suburban, middle-class upbringing of most students), several of these students reported "opting out" of academic and social environments in college. These students were more likely to describe their struggles with integration in terms of economic constraints or their different backgrounds than to attribute difficulties to the renegotiation of relationships with families.

However, while one-fourth of the students interviewed by Stuber (2011) felt "alienated" and marginalized by their college experiences, the majority did not. Her interviews revealed that half of these students were integrated into campus life and rarely felt feelings of marginality, a finding which counters much of the literature on firstgeneration students (London, 1989; Orbe, 2004; Rendon, 1992; Richardson \& Skinner, 
1992). Family relationships were downplayed in these students' discussions of their "fit" on campus; in some instances students pondered why a lack of family experience with college would be meaningful for student experiences. In another instance, a student described at length how he had found a sense of "home" and "family" on the college campus that he had never felt growing up. Importantly, Stuber (2011) emphasized the role that whiteness played in helping these students blend into college. These students, whom Stuber dubbed "integrated persisters," primarily grew up in blue-collar homes with stable incomes and communities that were predominantly white, and neither perceived their own backgrounds as disadvantaged nor were accustomed to feeling different from those around them. These students conceptualized diversity primarily in terms of race, and assumed that "first-generation" programs were primarily targeted towards people of color. Overwhelmingly, these students did not see themselves as marginal or in need of intervention.

The final group of students that Stuber (2011) interviewed were "resilient persisters," and some shared that their initial feelings of marginality and alienation on the college campus had been transformative for them, driving them to desire social change. These students reported feelings of isolation based on fears they were the only ones to come from unstable economic backgrounds. Sometimes these feelings were overcome by connections with other students from similar economic backgrounds, but at other times students could not find others they "fit" with, and began to articulate feelings of marginality through a discourse of difference. However, often this discourse was framed in terms of race: students highlighted the feelings of frustration and invisibility of 
"passing" for typical white college students. For these students, whiteness functioned as a potential risk, hiding their struggles, but this was overcome through connections to others like them and a growing sense of class consciousness.

In terms of academic integration, a common finding in the literature focuses on first-generation students' struggles relating to faculty. In a multi-institutional survey of first-year freshmen on campuses across the U.S., first-generation students were less likely to report feeling supported by faculty than their peers (Terenzini et al., 1996). These first-generation students also differed from traditional students in their reactions to perceptions that faculty were concerned with student development: perceptions that faculty were concerned with students' progress were associated with gains in reading for traditional students, but among first-generation students these perceptions were associated with lower reading scores. Similarly, Padgett and colleagues (2012) demonstrated that teaching interactions with faculty did not have the same beneficial effect for first-generation students they did for peers, rather, even interactions with faculty described as "good practices" were associated with lower levels of well-being among first-generation students.

Collier and Morgan (2008) offered one possible explanation for the differences between first-generation students: first-generation students may struggle more with understanding and meeting faculty expectations. Focus groups conducted with faculty, traditional students, and first-generation students revealed that first-generation students did not accurately interpret faculty's expectations about workload and priorities, understanding assignments, communicating with faculty, and solving problems as they 
arose. Collier and Morgan noted that first-generation students appeared less likely to master the student role and couched their findings in cultural capital, discussed in more detail below. Students' ability to master the role and integrate academically were limited not only by students' lack of understanding, but also to their reactions to feelings of difference in the college setting. As one first-generation participant in Collier and Morgan's (2008) study said,

Talking to anybody my whole first year was out of the question... It was so scary. I'd heard from my whole family, when you go to college it's a whole different class of people...I didn't talk to any of the professors (p. 439).

This student's response highlights how family relationships are foundational to integration, not only academically, but also socially (since the "different class of people" isn't necessarily specific to students or faculty). This response suggests fears of marginalization, based on the assumption that the people who populate a college campus are fundamentally different from the people one has known. Academic and social integration may be easier for first-generation students in institutional settings that are more homogenous, Bradbury and Maher (2009) noted: the Appalachian students in their sample attended a campus with students from similar backgrounds. These authors also noted the importance of pedagogy in supporting integration: most classes were small and discussion-based, and students reported feeling their perspectives, regardless of generation status, were welcomed and affirmed.

\section{Other Responses to Integration: Resistance and Border-Crossing}


RELATIONAL WORLDS OF FIRST-GEN STUDENTS

Often integration has been posed as a process with binary outcomes: students either "break away" and integrate into college life or remain connected to family and risk isolation on campus. First-generation students' perspectives reveal a more nuanced picture of the struggles to maintain a place in both worlds. Using a relational-dialectic perspective, Lowery-Hart and Pacheco (2011) conducted focus groups with firstgeneration college students to understand the opposing tensions these students faced in integrating into college. Students felt tensions within themselves (Do I fit in here? Do I remain separated from other students, either out of pride, or out of fear?) that highlighted potential feelings of marginality, but also suggested active resistance of elements of integration. In some cases, integration was not even a goal because college itself seemed at odds with students' identities. As one student said:

I need a job to provide for my family. I come to class, I do my work and go home. I don't have time for college. I hafta work, raise my kids. College ain't for me. Some dude will be talking about a party or a magician that was on campus. That ain't me. College ain't me. I come here because I take care of my family (Lowery-Hart \& Pacheco, 2011, p. 59).

Both family roles and employment were mentioned as part of the calculations students considered in deciding how much to integrate, but more important is the clear declaration of college as something antithetical to this person's entire sense of self. Rather than lacking the skills to integrate, this student rejected integration outright.

Other students may not resist the goal of integration, but may passively forego integration due to competing demands on their time. Choy's (2001) observation that 
first-generation students were more likely to identify as workers than students who worked is relevant here, as is the finding that first-generation students tended to work more hours and were more likely to work and live off-campus than their traditional student peers (Bradbury \& Maher, 2009; Orbe, 2004; Pascarella et al., 2004; Terenzini et al., 1996). As Richardson and Skinner (1992) found, the role of student was not always salient among the reference groups of first-generation students of color. In listening to students, these authors also noted how little continuous time these students had available for schoolwork. Much like the first-generation students in Collier and Morgan's (2008) sample, these students fit schoolwork into gaps in existing schedules that were already full of demands.

In contrast to assumptions that students must end or curtail relationships outside of school in order to integrate, some students found themselves placed into new, but valued "outsider" roles in their communities. Often these students served as border crossers, translating the college experience for members of their families and communities. Some first-generation students reported special treatment by family members on their visits home from college: being given special food or treated to shopping for school-related needs (Orbe, 2004). Several studies described the continued focus of family relationships to first-generation college students; "carrying” family was a common theme among students in Orbe's (2004) focus groups, as was creating a path for siblings to follow (Gofen, 2009). In one of the few examples of social work literature on the experiences of a first-generation student in social work education, Jan Carter-Black (2008) described her journey through undergraduate education, recalling 
I carried much more than just the things in my suitcases that day as I left home. I also carried the hope of my family all bundled up and neatly tucked away inside where no one else could see. I was keenly aware of my precious cargo (p. 113).

As the first in their family to attend college, many students reported feeling that their academic success had a bearing on the educational futures of family members. Among the students in Bradbury and Maher's (2009) study, over one-half maintained daily contact with families, and many of these students reported that their families' needs drove their choices of majors and eventual career paths. An analysis of Bui's (2002) reasons that students give for attending college also supported connections to and responsibility for family. First-generation students were more likely to report attending college so they could bring honor to their families and help their families out financially after college. They were less likely to report attending college because their siblings had also attended, and less likely to report that they were attending college out of a desire to leave home. Some students noted that their status as a college student was also salient to the larger community they came from; this was especially common for students of color in Orbe's (2004) sample. These students described both feeling like they represented their community as a student when in school and were regarded as a liaison to the world of college when in their communities. This student described his mother's insistence that he accompany her to work during visits home, and other peoples' reactions to him:

All the women will say, “Oh, you're so-and-so’s boy. You are so handsome. You go to college?"... They talk to you like you're not a regular person any more. 
They talked to you like you are — not a superstar — but like you came back from outer space or something. "So what was it like there?" (p. 141)

While family connections are not typically considered to be an advantage for firstgeneration students, some research suggested that first-generation college students may not persist in school in spite of family connections, but because of them. Anat Gofen (2009) interviewed Israeli college students who were the first in their families to attend higher education and found that students mentioned family more than school, community, teachers, or mentors, as a factor in their ability to get into higher education and remain. Specifically, these students cited their family's positive attitudes toward education, their relationships with parents and siblings (including acting as a role model for younger siblings), and values of solidarity, respect for parents, and achievement as the primary supports that helped them break intergenerational cycles of lower educational attainment and poverty. Rather than acting as a weight that held students back, these students were unanimous in voicing the importance of the role that family played in supporting their educational goals. Similarly, the first-generation Appalachian students in Bradbury and Maher's (2009) sample described the powerful "pull of home" (p. 276)

and the tensions they felt between home (family, friends, paid employment, and living off campus) and their lives at school. However, this "pull of home" did not represent unwanted obligations; in some cases, it was "home" that drove students to persist.

\section{Theoretical Frameworks: Integration and Reproduction}

Two theoretical approaches predominant within the literature on first-generation college students are Tinto's $(1975,1993)$ Theory of Student Departure, which focuses on 
academic and social integration, and Bourdieu's (1986) theory of social reproduction.

Tinto argued that the main driver of individual persistence is social and academic integration in the college setting. Bourdieu (1986) described "cultural capital" as a form of investment in an individual which is activated through schooling and converted to economic capital in the marketplace. Cultural capital exists in several forms: "embodied" in individuals' perceptions, mannerisms, values, and lifestyles (often referred to as habitus) as well as knowledge and abilities; "objectified" through both possession of culturally-valued objects such as books and art, and the knowledge or skills required to "consume" such materials; and/or "institutionalized," through the conferral of academic qualifications. One theory is focused on the individual's ${ }^{11}$ integration within an institution while the other posits that education is a means of reproducing social class from one generation to the next. I will turn to Tinto's (1993) theory first and offer a brief overview of each theory as well as critiques of each and alternate iterations posed by those who have found them lacking.

It is difficult to engage with the literature describing first-generation college students without encountering Tinto's (1993) Theory of Student Departure. Vincent Tinto's ideas are cited widely in the literature exploring first-generation students (Bradbury \& Maher, 2009; Cole, 2008; Collier \& Morgan, 2008; Richardson \& Skinner, 1992; Stieha, 2010; Stuber, 2011; Terenzini et al., 1996), reaching “near paradigmatic

\footnotetext{
${ }^{11}$ This point is open to debate. While Tinto (1993) refers to this as "A Theory of Individual Student Departure," others note that it is more apt for capturing retention as an institutional phenomenon. Renn and Reason (2013) offer Bean and Eaton's (2000) Psychological Model of Student Departure as an appropriate individual-level model for understanding persistence. However, it is notable that most of the studies below which cite Tinto apply these ideas at an individual, rather than institutional, level.
} 
status" (Braxton, 2000, p. 2) in the wider body of literature investigating student departure among all college students. Tinto's theory has also been critiqued for a lack of empirical support (Braxton, Sullivan, \& Johnson, 1997) and for assuming a model of college-going that is most appropriate for middle class white students and ignores racial and cultural differences (Guiffrida, 2006; Kuh \& Love, 2000; Tierney, 1999). As such, Tinto's theory often appears in studies accompanied by some discussion of its drawbacks (Cole, 2008; Collier \& Morgan, 2008; Richardson \& Skinner, 1992; Stieha, 2010). However, because questions about integration into the social and academic spheres of college are so prominent in the literature on first-generation students (Collier \& Morgan, 2008; Lowery-Hart \& Pacheco, 2011; Orbe, 2004; Stephens et al., 2014; Stuber, 2011), an introduction to Tinto's theory is necessary.

Tinto (1993) proposed his theory in response to psychological theories of departure which located the causes for leaving within individuals, instead focusing on students' institutional experiences. Tinto's theory began with the individual student, who in addition to having certain attributes prior to entry (family background, skills and abilities, and academic preparation through prior schooling) was also assumed to have certain goals and commitments. Tinto broke down these goals and commitments as intentions (students' educational or occupational aspirations), their commitment to those intentions, and their commitment to the institution. Tinto also considered the commitments students held that are external to their education. According to Tinto, goals and commitments were influenced through institutional experiences that impact the degree of academic and social integration. Students who failed to integrate socially and 
academically were more likely to voluntarily leave school. Academic integration involved both formal modes, through academic performance, and also informal pathways, through student interaction with faculty and staff. Likewise, social integration also has formal paths, through extracurricular activities, and informal modes, through interactions with other students.

In Tinto's (1993) Theory of Student Departure, academic and social integration hinge on two processes: separation and integration. Informed by VanGennep's (1960, as cited in Tinto, 1993) ideas about rites of passage, Tinto argued that students must separate from communities of the past: "such communities differ from college not only in composition but also the values, norms, and behavioral and intellectual styles that characterize their everyday life" (p. 95). Likening integration to Durkheim's (1951, as cited in Tinto, 1993) ideas about incorporation, Tinto posited that students who were able to adopt new norms and behavioral patterns that allowed them to become incorporated into the "social and intellectual" communities of the college would be more likely to remain in college. So why do individuals leave? According to Tinto, at an individual level, each student has expectations and motivations for schooling. During their time in higher education, each student engages in a continual process of assessing their own levels of academic and social integration, based upon feedback from formal and informal modes of academic and social engagement. Tinto argued "that some degree of social and intellectual integration and therefore membership in academic and social communities must exist as a condition for continued persistence" (p.120). 
Tinto (1993) saw the classroom as central, noting that social integration involves connection to a smaller group within the larger university. Because most students are non-residential, the classroom becomes a critical site for student engagement and integration. Tinto argued that instructional faculty must become as concerned with student retention as student affairs personnel, and felt that faculty have more influence in student retention because they are responsible for the creation of "smaller educational communities that serve as both gateways to and intersections for the broader academic and social communities of the college" (p. 133). Tinto cited Neumann and Neumann (1989, as cited in Tinto, 1993), whose study of academic persistence in juniors and seniors pointed to "accessibility and contact with faculty outside of class," "helpfulness of faculty," and "concern they show for students" as factors supporting persistence as evidence of the critical role faculty play in student retention.

As noted above, Tinto's (1993) Theory of Student Departure is not without its critiques. After reviewing empirical studies which tested the fifteen propositions (see Appendix A) implied by Tinto's $(1975,1993)$ model, Braxton and colleagues (1997) found moderate empirical support of the theory at best. Across studies of both single and multiple institutions, only two of Tinto's fifteen propositions found strong support: Item 10. The initial level of institutional commitment affects the subsequent level of institutional commitment, and Item 11. The initial level of commitment to the goal of graduation from college affects the subsequent level of commitment to the goal of college graduation. In particular, the concept of academic integration has found only moderate 
empirical support, and Braxton and Lien (2000) argued it should be revised, if not discarded altogether.

Others criticized Tinto's (1993) model on theoretical grounds rather than the lack of empirical support. The concepts of separation and integration may be particularly problematic for students who are underrepresented on college campuses. It is interesting to note that in his discussion of separation, Tinto (1993) cited London's (1989) study as evidence of the need for students to "break away" from communities of "past association" (p. 93), but failed to note that London's study might not be representative of all college students, since London was only writing about first-generation college students. Tierney (1999) argued that Tinto's (1993) model was inappropriate for application to students of color based on his ideas about separation and integration, arguing that students of color who are asked to separate from their communities and integrate upon entering primarily white institutions may experience a form of "cultural suicide" (p. 82). While Tinto (1993) said all students must separate from their communities, Tierney pointed out that this separation is not the same for all students. Rites of passage, the framework Tinto used to describe separation, typically involve a community member's movement from one status to another, but do not involve movement out of one community and entrance into a new one entirely. Students of color, Tierney noted, are more likely to be moving from one (non-dominant) community and into another (dominant) community. Therefore, the separation required of students of color may be markedly different than it is for students from the majority culture. 
Likewise, the presumption that students must integrate into the college community, which may reward values that are not representative of students' backgrounds (through an emphasis on individualism over collectivism, for example), rendered these students' backgrounds as unimportant and placed the responsibility for separation and integration fully on the individual student, regardless of how similar or different their backgrounds. Instead, Tierney argued for "cultural integration" (p. 84), which involved recognizing and honoring student backgrounds and a view of "the academy as ripe for reinterpretation and restructuring" (p. 83). Cole (2008) argued that social class, in addition to race and color, is important to consider in the process of integration.

Rather than abandoning Tinto's (1993) ideas, others proposed modifying his ideas to recognize the role of culture in students' experiences in college. Kuh and Love (2000) outlined several propositions with the recognition that students view the college experience through different cultural lenses, and bring different levels of knowledge about college with them when they enroll. Kuh and Love posited that cultural distance between students' culture of origin and the culture of higher education is related to persistence, and students experience stress when negotiating these distances. The authors described "cultural enclaves" as one means of mediating the distance between the cultures of home and school: groups that share attitudes, beliefs, and norms that are more congruent with students' home cultures. The authors proposed that cultural stress may be especially great for students who continue to remain in their cultures of origin after enrolling in college; these students may see college not as a new space to immerse 
themselves in but as an additional space to add to their existing social world. These students may face constant pressure to adjust their values, attitudes, and practices as they move back and forth between different cultural worlds. Guiffrida (2006) also proposed modifications to Tinto's (1993) Theory of Student Departure, adding home social systems alongside the academic and social systems of the university as parts of the process of integration. Guiffrida also pointed to the role that cultures play in shaping the pre-entry attributes of students: students from different cultures may bring different intentions, goals, and commitments based on the value their home cultures place on individual achievement, competition, and autonomy. For example, a successful student in higher education may be motivated by needs for autonomy, competence, and recognition through higher grades, all values prized in the dominant culture, but a student whose culture places more emphasis on collectivism than individual needs may feel at odds with the competitive nature of many college classrooms. What may appear to be differences in intentions, goals, and commitments may actually reflect cultural differences in which needs motivate students (Guiffrida, 2006).

Perhaps most useful is the examination of how "integration" is used in the literature (Kuh \& Love, 2000). While the word "integration" is typically defined as the combination of smaller parts into a complete whole, "integration" as used in Tinto's $(1975,1993)$ theory could be more accurately described as "assimilation" or “incorporation." Framing integration as a student's adoption of the values, customs, and attitudes of an institution suggests that students bear the full burden of integration - the 
RELATIONAL WORLDS OF FIRST-GEN STUDENTS

practices of the academy remain unexamined in terms of their responsiveness to the cultural backgrounds of students. 


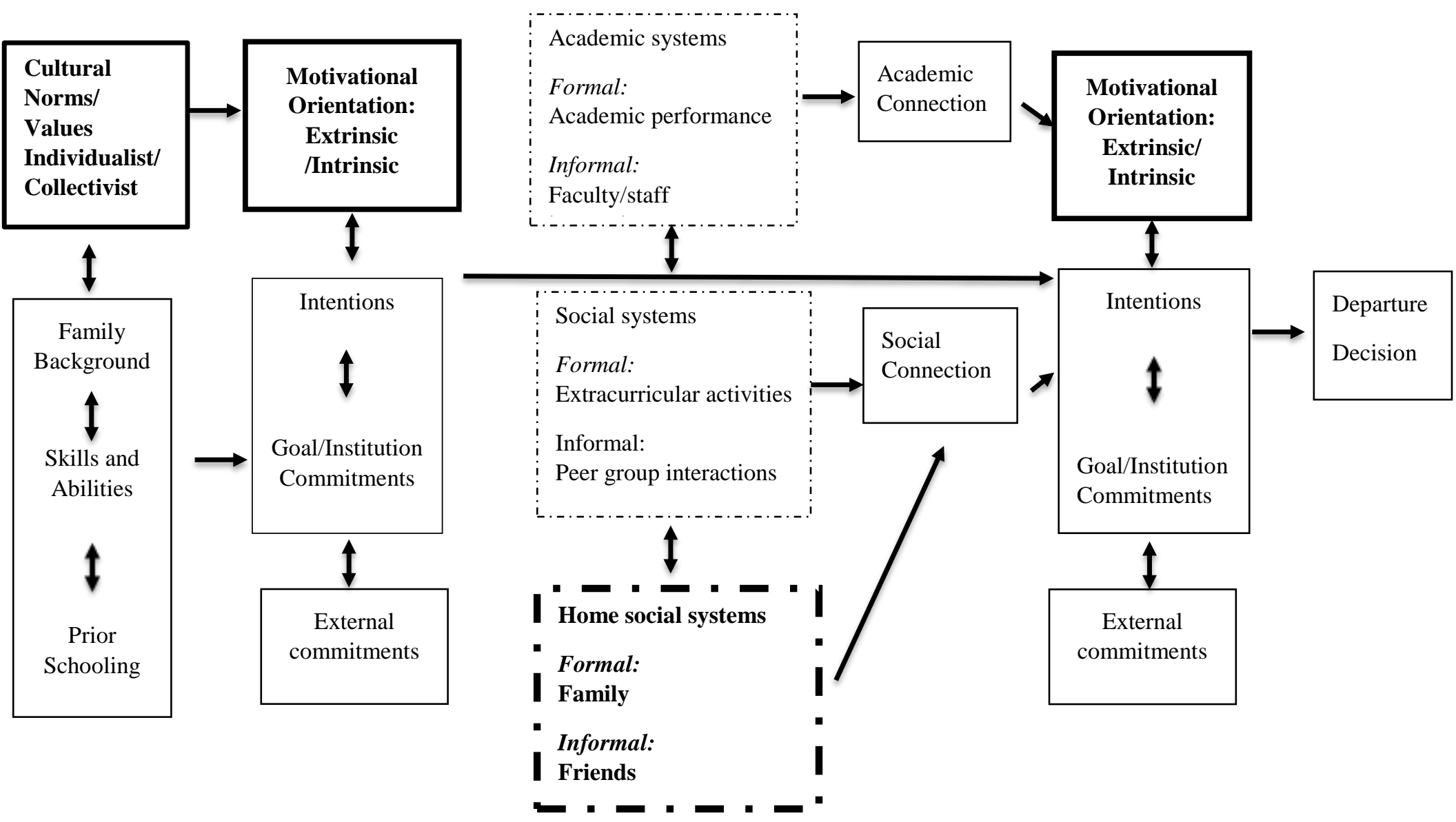

Figure 1. Guiffrida's (2006) Recommended changes to Tinto's theory (in bold). 
RELATIONAL WORLDS OF FIRST-GEN STUDENTS

Cultural backgrounds are central to Bourdieu's (1986) notion of cultural capital. Bourdieu proposed cultural capital as a means of explaining disparities in school success among students from different social classes, and his ideas appear frequently in the literature examining the experiences of first-generation students (Collier \& Morgan, 2008; Padgett et al., 2012; Pascarella et al., 2004, Stuber, 2011). Cultural capital is conceptualized as "privileges and attitudes" of the middle-class and upper-class (Padgett et al., 2012, p. 246) and comfort with the dominant culture (Collier \& Morgan, 2008; Pascarella et al., 2004). Cultural capital is a symbolic good with social value that exists in multiple forms (Bourdieu, 1986), but the embodied state is most germane to our discussion. Embodied cultural capital might manifest as talents, abilities, or knowledge in an individual: "goods" which are often the product of long-term investment of time and money. Embedded within his discussion of the embodied forms of cultural capital is Bourdieu's description of habitus: the attitudes, beliefs, and perceptions of an individual; the subtle "lens" through which one views the world, largely shaped by social contacts. The types of cultural capital valued by dominant classes are often rewarded in educational settings, although they are not explicitly taught in schools (McDonough, 1997). It was through this unequal valuing of cultural capital in educational settings that Bourdieu (1986) proposed the intergenerational transfer of class status is legitimized, as students with more "talent," "knowledge," and "ability" are retained or promoted in schooling, maximizing their chances of educational and occupational success.

Bourdieu's (1986) ideas about cultural capital are nested within a larger framework that assumes patterns of social reproduction. By outlining the non-monetary 
forms of capital and how these are transformed into economic wealth, Bourdieu described a process that involves individual-level interactions in organizational climates that contribute to societal-level patterns of social inequality that persist across generations. The notion of cultural capital is often employed exclusively at an individual level in studies of retention among first-generation college students. For example, a cultural capital framework has been used to describe the choices students make about colleges in terms of selectivity (Pascarella et al., 2004), student understanding of the value and importance of college (Padgett et al., 2012), and students' understandings of faculty expectations (Collier \& Morgan, 2008) in relationship to first-generation student success in undergraduate education.

However, Bourdieu's emphasis on the role of schools in solidifying the process of social reproduction begs for an examination of institutions in addition to individual students. Berger (2000) proposed that institutions, as well as families, have an investment in maintaining or promoting their level of cultural capital. Usually denoted in terms of selectivity, institutions tend to develop distinct organizational climates that are reflective of their own levels of cultural capital. While much less work has examined the roles that institutions play in promoting social reproduction, Berger pointed to a study that examines how coursework, curricula, and pedagogical practices operate to affirm students with higher levels of cultural capital and isolate or ignore the cultural capital brought by students form lower classes (Rhoads \& Valadez, 1996, as cited in Berger, 2000). Berger (2000), using Bourdieu's ideas about cultural capital and social class, argued that according to a social reproduction perspective, students from non-majority 
and non-dominant backgrounds should expect to face struggles in "integrating," since the institution will remain invested in its own agenda for social reproduction. That is, as part of the mechanisms of ensuring that cultural capital transfers from generation to generation unheeded, universities are designed to welcome some and make others feel marginalized. Student backgrounds are critical for integration in university, Berger argued, and students who integrate well do so because their background prepared them for it, not because they "separate" from those backgrounds, as Tinto (1993) suggested.

Furthermore, employing notions of cultural capital strictly at the individual level has the effect of obscuring rather than highlighting existing social inequalities (Levitas, 2004). The meaning of cultural capital varies depending on the discursive context, but in education, cultural capital is often used to explain educational inequalities as the result of deficiencies in individual students and families, rather than structural inequalities. Thus, interventions are often focused at the micro level and legitimated through discourses of eventual financial payoff - that is, it's important to include those who have historically been excluded from education because it should result in a long-term decrease in the number of individuals who will later require societal investment through safety net programs (Butler, Deprez, \& Smith, 2004; London, 2006). I do not mean to suggest that investment in higher education is unnecessary, and I strongly oppose the withdrawal of educational supports in favor of "work promotion" that has been the result of welfare reform (Johnson, 2010). But by keeping the focus on individuals (particularly presumed deficits of the poor) and wielding cultural capital purely as a means to an economic end, 
class domination is concealed, rather than highlighted as Bourdieu (1986) originally intended.

Whether the focus is on the role schools play in social reproduction or on individual students, the student is often assumed to have little to no agency (McLeod, 1995). McLeod highlights Giroux's (1983a) theory of resistance as a means of attending to student behaviors that occur in response to experiences of schooling. While not all acts of student resistance arise from a critique of schooling practices, Giroux (1983b) asserted the importance of recognizing when students' actions are responses based in “moral and political indignation" (p. 289). Too often, students' oppositional behavior and/or resistance is ignored or attributed to dysfunction on the part of the student, but Giroux (1983b) argued that students should be allowed to explain resistance and contextualize their behaviors in the meaning-making systems of their peers, family, and work, in addition to the classroom.

\section{Summary and Locating First-Generation Students in Social Work Education}

It's clear from the literature that many first-generation college students face some struggles in adjusting to college. These struggles are often framed in terms of academic achievement, persistence, and retention. However, as London (1996) contended, the picture is much more complicated. Many first-generation students complete college with no signs of difficulty, particularly those who see themselves as beneficiaries of upward mobility made necessary by increasing demands for education and the broadening of access to higher education. Because of increasing levels of educational attainment among the general population, first-generation students may see themselves as part of a 
larger trend which has little to do with their family's educational background.

For many first-generation students though, the process of higher education is transformational (London, 1996). Transformation in itself is not inherently positive or negative, but may play out in "intellectual, psychological, family, and cultural dramas" (London, 1996, p. 9) which place demands on students that extend beyond the traditional work of college. London found that intellectual growth radicalized some students, but more students experienced non-political changes that resulted in feelings of enhanced competence. At the same time, students often underwent changes in self-concept and identity as they considered how education changed them. Some first-generation students tried on seemingly insignificant markers of identity (clothing, music or art, food, political ideology, leisure activities, etc.) which, taken together, served as subtle markers that denote class differences in a society where discussions of class are taboo (Liu, 2011). Whether students saw themselves as class mobile or not, for many completing a college education involved a transformation that demanded that first-generation students renegotiate not only their sense of who they were as individuals, but who they were in relation to family.

First-generation college students in the midst of transformation may find themselves at odds with the expectations of the role they should fulfill as a student, and may also struggle with seeking assistance. Lacking a familial template for the model of "college student," first-generation students tended to react to faculty in different ways than their traditional student peers. For example, while traditional students benefitted academically from contact with faculty, contact with faculty was associated with declines 
in performance for first-generation students. First-generation students also expressed conflicting feelings about fully embracing the student role, and their ability to do so was further complicated by higher levels of employment, more extensive caregiving responsibilities, and the need to study harder to overcome secondary educations that failed to adequately prepare them for the rigors of college (Reid \& Moore, 2008). Roles as an employee and/or care provider may be much more pressing than responsibilities as a student. For some students, aspects of college life that signal integration, such as participation in athletic, cultural, social, or intellectual activities seemed trivial and unappealing In light of these considerations, it is little wonder that numbers of firstgeneration college students may not feel academically or socially connected to campus (although as Stuber (2011) pointed out, many do feel "integrated"). The question of what meanings first-generation students make of these struggles to balance multiple social worlds is relatively unexplored.

The literature is fairly consistent in documenting high levels of contact with family for first-generation students, although this contact tended to look different. London's (1989) theme of "breaking away" aligned with assumptions posed by Tinto (1993) that successful integration into college requires that students separate themselves from family. Many students, however, stay close to families and negotiate feelings of rejection by their families, feelings of rejection of their families or their families' cultural values, or the not entirely unproblematic status of "hero," someone who may be both honored as an insider and outsider at the same time. These students may experience some of the cultural stress that Kuh and Love (2000) proposed is common to students 
who frequently move back and forth between the worlds of family or work and college. Also important to note, though, are the first-generation students who communicated their family's importance in their own education; for many students, their education is completed for their families and/or their education may be completed because of their families' contributions. The tensions in relationships to family suggest that many firstgeneration students cross "borders" between family and community and school. While some felt valued communicating their new role as a student, others experienced marginality in multiples spheres: some first-generation students reported that their transformations rendered them feeling like they didn't fully fit in with family or in school (London, 1989; Lowery-Hart \& Pacheco, 2011; Rendon, 1992).

Because first-generation status has been associated with lower levels of retention, Tinto's (1993) Theory of Student Departure is often employed as a means of supporting first-generation college students in completing higher education. Even when Tinto's model is not explicitly used, several interventions operate on an assumption that firstgeneration college students need support in integrating into the college campus, and are focused in particular on academic integration (providing tutoring and support, for example). However, pursuing the goal of integration may be problematic in several ways. Integration places the burden of change entirely on the student, overlooking ways that the institution may play a role in reinforcing hierarchical relationships and prizing certain class backgrounds and knowledge over others, as proposed by Bourdieu (1986). It is exactly these oppressive relationships which social work, in particular feminist social work, is equipped to name, and ideally, transform (Saulnier \& Swigonski, 2006). 
A second problem with the concept of integration is that it is often taken for granted that students want to integrate into the college campus. The literature suggests that this is not always the case. For some first-generation college students, their new identification as "student" may be enough to set them apart from family and/or community in ways that may be troubling. Integration into campus life or deeper identification with the student role may mark these students as moving further apart from their families of origin, and for some that distance is unmanageable. As Tierney (1999) pointed out, not all separations are the same for students when they enter college.

Finally, the concept of integration, although often used in conjunction with Bourdieu's (1986) ideas about cultural capital, ignores the conclusions from Bourdieu's theory which suggested that habitus develops slowly over time and cannot be separated from an individual or developed second-hand. Those students who step into the college environment and feel at home do so because their backgrounds prepared them for it, usually over a long period of time, and interventions which are short-term in nature may not be adequate enough to provide the same kind of intense investment typically involved in the building of cultural capital. While I am not arguing against interventions in support of first-generation college students, I do believe those who seek to intervene should consider the goal of integration carefully, including the importance that students attach to this goal. It's also important to note the possibility of further stigmatization that may arise through interventions which focus on the real or perceived educational deficits of participants. 
Understanding both Tinto's $(1975,1993)$ and Bourdieu's (1986) ideas are important because of their widespread use in the literature, and it seems difficult to escape their influence in thinking about first-generation college students. Separation from family and community is an a priori assumption in Tinto's theory, and Bourdieu's ideas about cultural capital seem to imply that a lack of family experience with college is a deficit. Both of these theories, at least as used in the literature, construct the student largely as an autonomous individual. However, as noted earlier, first-generation status is by definition relational. These theories, particularly Tinto's theory, also imply a causal template to relational issue: the literature offers evidence of students who separate and integrate (or fail to), as well as students who resist integration and students who remain actively involved in their families and communities. Relationships, particularly family relationships, are central to all of these responses, even (or especially) those that involve separation.

Rather than examining the effects of first-generation status on students, in this research I am interested in the relational worlds of first-generation students. Gergen (2009) argued that our identities are created in relationship with others: we do not enter into and exit relationships with identities unchanged, instead our identities are constructed in co-action with one another. Our behaviors, speech, and thoughts are influenced through contact with others, significant and otherwise, and are contextspecific and validated by those around us. Gergen (2009) noted that it was "remaining intelligible within our relationships" with others that "holds civilization together" (p. 140). Enacting the role of "student," "social worker," or other professional helper 
involves engaging in behaviors and embracing ideas particular to the college setting. What does this mean for first-generation students in a school of social work? How do students make sense of their experiences in school and "remain intelligible" within their relationships outside of school, if taking on the role of student, social worker, or member of the helping professions involves a change in speech, values, or behavior?

It's also important to note the lack of a sense of group identity among many firstgeneration college students. First-generation students varied widely in how much importance they attached to their status as "first-generation," and multiple researchers noted that students weren't aware "first-generation" status meant anything until they were approached regarding research on the phenomenon they were unwittingly experiencing. Even for students who were aware, first-generation status might mean very little; for students who faced multiple oppressions (sexism, racism, and classism), first-generation status tended to be much more meaningful. However, the aforementioned stigma attached to this identity, or other aspects of first-generation students' identities may make students hesitant to participate in interventions targeted for them.

The gaps in knowledge regarding first-generation students in social work are significant. A single study exists that provides demographic information for an undergraduate social welfare program at a single university (Hodges, 2000). The Council of Social Work Education's annual survey of social work programs offered insights into the basic demographics of the population of students enrolled in baccalaureate (BSW), master's (MSW), and doctoral programs in social work, but first-generation status of 
students was not covered (CSWE, 2012). For social work students, the first-generation experience is relatively unexplored.

The Annual Statistics on Social Work Education in the United States provided a demographic picture of social work programs across the United States. For full-time students in BSW programs, the majority were white $(50.6 \%)$ women $(87.5 \%)$ under the age of 25 (CSWE, 2012). Part-time BSW students were also overwhelmingly women (86.1\%), but showed greater racial and ethnic diversity and a wider range of ages. These students were more likely to be over 25 than younger than 25. Among students enrolled full-time in MSW programs, most tended to be women (85.7\%) who were under age 35 (73\%). The majority of these students were white (56.6\%). While part-time MSW students were more likely to be older, white students (53.9\%) and females $(85.4 \%)$ were still overrepresented among part-time MSW students.

Even in the absence of statistics documenting the numbers of first-generation students in social work programs, some important observations relevant to firstgeneration students can be made from the scant literature on the experiences of social work students who are people of color. Bowie and Hancock (2000), in an attempt to understand reasons behind the decades-long trend of stabilized and/or declining numbers of African-American students in graduate-level social work education, assessed students' influences on their career choices on two campuses in a southeastern city. An important aside noted in this study was the prevalence of experiences with racism in schools of social work during their graduate education: almost $75 \%$ of students said they had encountered institutionalized racism on their campus and over $60 \%$ of students said that it 
had affected them personally. Faculty sensitivity to racism was fairly low, with $29.2 \%$ of students reporting that there was "a little" support and 15\% reporting that it was “nonexistent." After a racist encounter with another social work student in a class discussion in her MSW program, Davis (2004) provided an honest account of the pain she felt as a person of color when a racial epithet was met with silence and a lack of action by fellow students, the professor, and program administrators.

Perhaps the most compelling description of the struggles of students of color in social work was offered by Daniel's (2007) qualitative interviews with 15 students in a graduate social work education program. African American and Latino/a students described experiences of cultural and racial isolation due to a lack of students and faculty of color in their program. This led to less informal support (through relationships with peers who might share their experiences) and a lack of professional mentoring. An overall lack of attention to race and diversity in graduate social work education meant that experiences of discrimination in classrooms (such as a student's racist remark that went unnoticed, unnamed, or unchallenged) and in faculty interactions (such as encountering instructors who had low expectations for the work of students of color) went unaddressed. This lack of attention to race included curriculum which didn't reflect the experiences of students of color, leaving students who had hoped to return to their own communities as social workers feeling unprepared due to the lack of a culturally relevant curriculum. Part of this arose from the lack of the voices and experiences of people of color in the literature used - when people of color were mentioned in classroom materials, too often the ones doing the describing were white voices describing a 
racialized "Other." Students of color also mentioned a lack of awareness of issues related to racism in their field placements by white field instructors, but not field instructors of color. While the experiences of first-generation students were not explicitly highlighted in social work accounts (except Carter-Black's (2008) narrative, in which she identifies as a first-generation student), the overrepresentation of students of color among firstgeneration students suggests that these experiences may be meaningful.

The literature on experiences of social work students who have experienced poverty or economic stress is even more limited. Searches of the Journal of Social Work Education, the Journal of Baccalaureate Social Work, and the Journal of Teaching in Social Work using the search terms "low-income students," "low SES students," and "working class students" resulted in zero results. Similarly, searches of Proquest Social Services Abstracts using the terms "social work education" and "low-income students" and "low SES students" resulted in zero matches. Common themes in the literature focused on social work education included student attitudes towards poverty and perceptions of people in poverty (see for example Weaver \& Yun, 2011), pedagogical approaches to educating social work students about poverty (see for example Gray \& O’Neill, 2013; Strier, Feldman, \& Shdaimah, 2012; Vandsburger, Duncan-Daston, Akerson, \& Dillon, 2010; Zosky \& Thompson, 2012) and explorations of which factors drive social work students to want to work with those in poverty (Perry, 2009).

Given the lack of studies focusing on low-income students' experiences in social work education, it is difficult to draw conclusions about the experiences of firstgeneration students in social work (who may also be more likely to come from low- 
income backgrounds, as demonstrated in the demographics of first-generation students). However, social work is certainly not immune to experiences of classism. Liu (2011) asserted that experiences of classism are common in the helping professions, in part due to taboos regarding discussions of class and inequality in our society and in part due to imprecise measurements of class. Understanding experiences of first-generation students in social work who may also have experiences with poverty and/or classism is critical to supporting and retaining diverse social workers.

As in other professions, the process of professional socialization as a social worker asks students to identify with attitudes, values, and interests of the larger profession (Barretti, 2004). Qualitative research with social work students has revealed important questions about the process of professional socialization. Fran Wiles (2013), in her work with students in the UK, found that constructing a professional identity as a social worker involved developing certain traits (expert knowledge, ability to practice with relative autonomy, understanding boundaries and social work values). Social work students also constructed identities as social workers through identification with social workers as a collective, and on an individual level, as their sense of their professional identity emerged. Barretti (2004) has called for similar qualitative explorations of the process of professional socialization for social work students in the US, arguing that unlike the medicine and nursing fields, which are populated with many exploratory, qualitative studies of professional socialization, social work examinations of professional socialization have relied heavily on one-dimensional measures of attitudes and values 
which are reminiscent of a psychological approach to human behavior and leave many questions about socialization unexplored.

What we do know is that many social work students of color share experiences of marginalization in social work education that impact professional socialization. The lack of attention to racism shown by many white field supervisors is especially meaningful in the process of socialization for social work students of color (Daniel, 2007). Over time, students of color reported increasing feelings of pressure to ignore or overlook experiences of discrimination and racism which occur in field placements when their field instructors were white, as part of developing a professional identity. In essence, these students were asked to disassociate themselves from their own communities and become blind to racism. The author suggested that schools of social work need to prioritize hiring and retention of faculty and students of color, and attend to curriculum as important supports in the retention of a racially diverse group of social workers.

Even less is known about students from low-income backgrounds in schools of social work and their experiences of the process of professional socialization. However, based on the findings that first-generation college students struggle with relationships with faculty, and the important role that social interaction with faculty members plays in the process of professional socialization (Barretti, 2004), it stands to reason that students who don't see their cultural, racial, and/or class backgrounds validated in higher education during the professionalization process are at a distinct disadvantage.

The experiences of first-generation students in schools of social work are important not only because of social work values of social justice, dignity and worth of 
the person, and human relationships, but also because of the now decades-old professional commitment to recruit and retain a diverse group of social workers (Casstevens et al., 2012). This study will fill important gaps in the literature about the ways that first-generation students in social work negotiate relationships with family, community, and school and the implications this may have for the professional socialization process. 


\section{CHAPTER 3: METHODOLOGY}

Because this study was designed to highlight the voices of first-generation student in a school of social work and their constructions of their relational worlds, I chose to employ a feminist approach, which Reinharz noted (1992, as cited in Eichler, 1997) “... aims to create social change; strives to represent human diversity; frequently includes the researcher as a person; attempts to develop special relations with the people studied (in interactive research); and frequently defines a special relationship with the reader" (p. 12). Attention to power relations and difference are prominent within much feminist research as well as attempts to decrease hierarchical relationships, and feminist research, more than other approaches, acknowledges political positions and the value of claiming these positions. Feminist approaches to research are not defined by distinct methods, but tend to direct considerable attention to methodology and epistemology (Campbell \& Wasco, 2000; Eichler, 1997; Strega, 2005). The body of literature that has sprung from feminist writing on research is vast, so in this section I'll attempt to include a broad overview of epistemological positions, taking a deeper look at traditions I've aligned this research within, and then discuss the implications for a methodological approach.

While social work has deep historical connections to the development of feminisms ${ }^{12}$, feminist approaches are less widely represented in social work research than in other fields (Wahab, Anderson-Nathe, \& Gringeri, 2012). Responding to Wahab and

\footnotetext{
${ }^{12}$ While the term "feminism" is often used, feminisms more accurately denotes the various strands of feminist thought which include but are not limited to: liberal, postmodern, Third Wave, radical, Marxist/Socialist, Postcolonial/Third World, and Black Feminist thought (J. Muthanna and S. Leotti, personal communication, Nov. 23, 2010).
} 
colleagues' urging to social work scholars to engage with theory more deeply, and acknowledging the limitations of social work research on this point, I will draw from works in anthropology (Behar, 1996), community psychology (Campbell \& Wasco, 2000; Fine, 1992; Fine \& Weis, 2005), education (Giroux, 1991; Lather \& Smithies, 1997; Reay, 2012), sociology (Collins, 2000; Eichler, 1986,1997), and where possible, social work (Morris, 2006; Presser, 2005) in this discussion of feminist approaches to research. Others have shaped these debates from law, philosophy, science, and/or feminist theory in general (Crenshaw, 1995; Harding, 1993; hooks, 2000).

Although her scope was limited to qualitative feminist research, Oleson (2005) argued that the central theme in feminist research is knowledge: whose knowledge is valued, how is it sought and by whom, and who sets the agenda for research? While the dominant stance in research presumes that researchers are objective and impartial observers, feminist approaches to research recognize that the relationship between the researcher and knowledge is not inconsequential. Campbell and Wasco (2000) combined various feminisms articulated in academic literature (liberal, socialist, radical, and womanist) and basic epistemological positions (positivism, realism, critical theory, and constructivism) to sketch three feminist epistemologies: empiricism, standpoint theory, and postmodernism. Feminist empiricism will be briefly introduced here before I engage in a more thorough discussion of standpoint theory and postmodernism. According to Campbell and Wasco, feminist empiricism combines post positivist realism with interests and values common to liberal or second-wave feminism. Feminist empiricists critiqued positivist science for persistent androcentric biases in research questions, study design 
and methods, interpretation of sex differences (or the under-interpretation of similarities), and broad generalizations from samples including only men. Eichler (1986) categorized much of traditional social science as "sexist science," noting that attention to sex bias was also important in language, concepts, and policy recommendations. Because of their alignment with a realist view of the world, feminist empiricists tended toward use of traditional research methods and designs, but carried out this research with attention to possible sources of gender bias (Campbell \& Wasco, 2000). Feminist empiricists arguably played a large role in highlighting the prevalence of sexist bias in supposedly "neutral" and "objective" work (Harding, 1993), but like liberal feminism, focused on issues of equality and access that stopped short of reforming major social institutions.

\section{Feminist Standpoint Theory}

Campbell and Wasco (2000) described feminist standpoint theory as a combination of the epistemological positions of critical theory and multiple forms of feminisms (radical and socialist, as well as womanism). Owing to the ontological claims of critical theory (Guba \& Lincoln, 2005), standpoint theorists argued that there was not a single, objective "truth" to be discovered, but instead that our understandings of reality are shaped by aspects of our identity: class, race, gender, and sexual orientation (Campbell \& Wasco, 2000). Importantly, standpoint theorists paid careful attention to the social location of those making knowledge claims. Because of the unequal distribution of power and resources in society and the role power plays in determining who has the authority to make knowledge claims, standpoint theorists have argued for attention to the views of those with less power. Harding (1993) offered Hegel's 
exploration of the differences between viewing the master/slave relationship from the viewpoint of the slave rather than the master: while the master is more likely to have the authority to produce an account of the relationship, the slave's perspective probably provides a more accurate depiction. Because of differences in power and authority, the master is able to ignore and overlook subtleties (and circumstances not so subtle) that the slave must attend to closely in order to survive. Standpoint theorists contended that research beginning with the perspectives of those who are marginalized is more likely to produce a more accurate view of social reality (Campbell \& Wasco, 2000; Harding, 1993; Oleson, 2005).

Flowing from a recognition of structural oppression and their impact on lived experiences, as well as a foundation in critical theory (see for example Kincheloe \& McLaren, 2005; Morris, 2006), feminist standpoint theorists maintained that research with groups that experience oppression(s) has the potential for producing research that critiques oppression in existing social relations, but recognizing this potential requires consciousness-raising among participants. Here we see clear connections to Freire's (1970/2000) Pedagogy of the Oppressed, which outlined dialogue as a means of "revolutionary leaders" learning with and from others (the "oppressed") to understand "both their objective situation and their awareness of that situation" (p. 95, emphasis in original). Freire described the experiences of oppression in peoples' lives as "limit situations," and argued that it was the perceptions of "limit situations" even more so than the actual "limit situations" themselves that can inspire courage and hope for change, specifically liberation from oppressive "limit situations." Similarly, Campbell and 
Wasco (2000) claimed that feminist standpoint theorists must engage participants in reflection on their experiences and the roles that class, gender, race, and sexual orientation may play in shaping their social worlds.

At this point it is important to pause and note an important divergence in ideas about power. Collins (2000), noting the "intersecting oppressions of race, class, gender, sexuality, and nation" (p. 291-2), argued that it is not enough to change the consciousness of individual Black women, but that unjust social institutions must also be changed. According to Collins, power is conceptualized in different ways. Those who see power as existing in a dialectical relationship between those with more power and those with less power espouse a theory of activism in which understanding can lead to group-based resistance to oppression on the part of those who are oppressed. Freire's (1970/2000) notion of the more powerful "oppressors" and the need for consciousness raising among the oppressed is reminiscent of this description of power. However, Collins argued, oppression is not merely an experience that exists in the mind, but is also an embodied experience that is always in flux. In contrast to a group-based notion of power (or powerlessness), Collins outlined an individual-based description of power as "an intangible entity which circulates within a particular matrix of domination and to which individuals stand in varying relationships" (p. 292). This subjective approach to power highlights individual agency and the ways individuals are both impacted by and resist domination. Collins did not argue for an abandonment of group-based consciousness, but instead for the inclusion of individual perspectives on power, noting that different aspects of our identity are more important (and offer more or less power) in different situations, 
depending on the context. In a similar vein, hooks (2000) critiqued those in the feminist movement who narrowly defined power as the ability to dominate others and assumed that sexism rendered women powerless in all situations. This view of women as powerless was based primarily in white, middle-class experiences of patriarchal relationships which offered some level of economic and physical security to women but afforded women little power. hooks pointed to the power exercised by lower class and working class women, particularly women of color, in order to survive independently, as evidence that sex alone was not determinative of power. Importantly, hooks argued that power was not based in the ability to move from a position of submission to domination, but in the ability to end domination.

Both Collins (2000) and hooks (2000) highlighted an important understanding within feminist standpoint theory. As Oleson (2005) noted, standpoint feminism dislodged the liberal feminist vision of the universal experience of women, exposing differences between women based on class, color, disability, ethnicity, immigration status, race, religion, and/or sexual orientation. This attention to the intersectional nature of various aspects of identity was outlined by Crenshaw (1995), who showed how dominant narratives of both overcoming sexism (as a struggle focused on the needs of white women) and overcoming racism (as a struggle focused on the needs of men of color) ignored the experiences and needs of women of color, who existed at the intersection of both oppressions but were not adequately represented by either movement. Crenshaw drew from legal records and women's experiences with social service organizations to demonstrate the ways that the "margins" served as a space where 
violence against women of color went unnoticed. Feminist standpoint theory required not only attention to differences within groups, but also differences in the researcher's identity and the ways that social locations might shape their understandings of the research process (Campbell \& Wasco, 2000). Harding (1993) developed the notion of "strong objectivity," which asked researchers to identify their values and place "the subject of knowledge... on the same critical, causal plane as the objects of knowledge" (p. 69).

\section{Feminist Postmodernism}

Finally, Campbell and Wasco (2000) claimed that feminist postmodernism (also called post structural feminism (Strega, 2005)), drew from the epistemological positions of constructivists, who reject notions of truth and reality, and the interests and values of radical feminists. This epistemological stance suggests a vision for feminist research which upends the core assumptions of science, making feminist postmodernism strikingly different from the first two forms of feminist research discussed. Strega (2005) noted that post structuralism challenged the thinking born of the Enlightenment-era which had structured dominant Western ideas about knowledge for centuries, giving "objective," "rational," and "impartial" science prominence over other ways of knowing (folklore, philosophy, or poetry, for example) and undermining the dualism inherent in this worldview: mind/body, self/other, subject/object. Because of this rejection of the scientific method, postmodern feminism and post structural feminism rely instead on careful attention to texts, in particular how history and power shape language. Oleson (2005) wrote that the postmodern shift in feminist research signaled an emphasis on 
objects of culture and meanings, and "discourse, narrative and text, and experimental writing of standard research account presentations" (p. 247). Lather and Smithies' (1997) account of focus group research with women living with HIV/AIDS offered an example, with a split-text format that displayed the researchers' notes and reactions on the page alongside transcripts of group interactions, making the research process more explicit.

Alongside attention to language, postmodern feminism also emphasizes the relationship of the researcher to participants. "Nothing is stranger," wrote Ruth Behar (1996) in a collection of essays on her anthropological work and her struggle to position herself in relation to the observed, "than this business of humans observing other humans in order to write about them" (p. 5). Arguing for the importance of reflexivity, Behar asked anthropologists and other academics to make clear their own position in relationship to social research. Lois Presser (2005) attended to these tensions between researcher and researched in her examination of the ways that race, class, and gender shaped power dynamics in interactions with men who were incarcerated. In research, there are often power imbalances between researchers and their participants, but Presser highlighted the shifting nature of power in her interviews: her relative race and class privileges offered power, but hegemonic discourses of masculinity enacted by her participants, which vacillated between offers of protection and threats of domination, also reminded her of the ways she had less power than the men she interviewed.

Feminist postmodernism has implications for subjectivity as well and the researcher's relationship to the research itself. Kamberelis and Dimitriadis (2005), describing Lather and Smithies' (1997) text Troubling the Angels, wrote “...Lather came 
to realize the ways in which knowledge is always already embodied - bodily, viscerally, and materially - and the consequences such a realization has for fieldwork and writing" (p. 901). For Lather, this meant that the research pushed her to reconsider her own feelings about loss, but the relationship between the researcher and researched extends beyond the intimate and personal into the political. Fine (1992) contended that all research is inherently political (Rosaldo, 1989), and therefore we must acknowledge our own interests and values in relationship to research. Fine described three positions one can take in research: "ventriloquism" (the neutral stance of a dispassionate observer), "voices," which Fine argued is a more subtle form of ventriloquism in which researchers "allow" the voices of (typically marginalized) others to speak but in doing so mask their own productive role in research, and activism. Activism is different from other forms of social research in three ways: the author's clear statement of politics and interests, attention to a critique of unjust social relationships, and a narrative that "reveals and invents disruptive images of what could be" (Lather, 1986, as cited in Fine, 1992, p. 221).

Postmodern thought is not without its detractors, however. Giroux (1991) wrote of the postmodern challenge to modernist notions of cultures as separate entities, marked by borders, and the implications for "border crossers"; if cultures are not actually distinct and bounded but instead social constructions that are always in flux, what does this mean for those who find themselves at the nexus of multiple cultures? Although borders and cultures are socially constructed and fluid, that does not mean these play out as mere “constructions" in lived experiences. Likewise, identity is a combination of social constructions which are lived in the body. Race, class, gender, and nationality are all 
social constructions with real implications for daily lives. Postmodern thought, through its reconceptualization of individual subjectivities not as stable, fixed identities but as multiple, shifting, and transitory, also threatens the potential for group identity and action (Collins, 1998, as cited in Oleson, 2005; Giroux, 1991). Springer (2002) summarized the destructive potential this way:

Just when I was discovering what each aspect of my identity had to do with all the others, postmodern theorists (mostly white, European men) tried to suck all the damn air out of the room and tell me there was no "I" (p. 384, emphasis in original).

The dissolution of group-based identities is particularly important for the study of firstgeneration college students, as Bourdieu's (1986) concept of cultural capital is rooted in a hierarchical model of social classes which are based on difference and assume some aspects of group membership.

However, even given the potential drawbacks inherent in postmodern thought ${ }^{13}$, feminist researchers have also realized the possibilities, often in tandem with standpoint theory. Sandra Harding (1996, as cited in Oleson, 2005) wrote that these "... approaches have been especially helpful in enabling standpoint theories systematically to examine critically pluralities of power relations, of the sort indicated in the earlier discussion of gender as shaped by class, race, and other historical cultural forces and how these are disseminated through 'discourses' that are both structural and symbolic" (p.246). Giroux

\footnotetext{
${ }^{13}$ Including, as Campbell and Wasco (2000) noted, the actual negation of postmodern research itself which is, after all, dependent on language which is always subjective and can never be free of the assumptions of its social and historical location.
} 
(1991) argued that feminism politicizes and recovers postmodern thought, and that postmodern feminism recognizes difference while rejecting essentialist thought, and provides a language attentive to inequality and the reciprocal relationships between individual human agents and larger social structures, wherein the possibilities for social change exist.

The foregoing discussion of divisions and commonalities in approaches to feminist research (empiricism, standpoint theory, and postmodern/post structural feminism) is critical for locating the current study as a project that is designed to attend to difference, power, reflexivity, and the need for social change. These considerations reflect several of the core values of social work as a profession. Sensitivity to difference is linked to social work's value of the dignity and worth of each person, attention to power as well as social change reflects social work's commitment to social justice, and reflexivity mirrors the social work understanding of the value of human relationships.

The stereotypical college student described in the introduction, "John," while becoming less common, is still the model for college-going assumed by many in higher education. When attending to difference and identity, an intersectional analysis is critical to avoid missing the differences within groups (for example, Stuber's (2011) examinations of the ways that whiteness masks economic need among working class first-generation students). First-generation college students are not a monolithic group, but are often spoken of as a bounded class, marked primarily by academic need and deficits. Postmodern feminism, through its ontological roots in constructivism, offers exciting possibilities for the exploration of the ways that identities are co-constructed in 
conversations among first-generation students (many of whom, as the reader will recall, lack any sense of group identity or even identify as "first-generation"). However, I agree with Collins (1998, as cited in Oleson, 2005) and Giroux (1991) that a strict reading of postmodern thought that disavows the possibility of identities shaped on the margins and considers an array of difference divorced from considerations of power is destructive and inaccurate. Identities are social constructions negotiated between ourselves and others around us, as well as in our relationships to larger institutional and systemic structures, and as such, are shifting and fluid. However, identities are also embedded with differing levels of power (including institutional power and our own power as agents), and relative levels of power can and do mark our lives. Fine and Weis (2005) articulated the resolution of these tensions most clearly for me, rejecting the notion of social identities as homogeneous categories that are stable and fixed in the body, but recognizing social identities as embodied in our relationships to one another and institutions, and marked by economic and political inequality:

You can't simply hang out in poor and working-class communities, a suburban mall, a prison, or an elite suburban golf course and come away believing that race, ethnicity, and class are simply inventions. Thus, with theoretical ambivalence and political commitment, we analytically embrace these categories of identity as social, porous, flexible, and yet profoundly political ways of organizing the world (p.67). 
Importantly, these authors noted the tendency for many of us, in understanding and describing our own social locations in relation to others, to essentialize those groups we don't belong to.

The sensitive reading of power (and the complex nature of power relations) afforded by feminist approaches is critical to this work. I see value in Freire's (1970/2000) reading of power as embedded in institutions and the importance of working with those who are marginalized by current economic and political structures and yet I also agree with Collins (2000) and hooks (2000) that any reading of power that overlooks human agency and the multiple ways we resist, challenge, or are complicit in oppression is incomplete. Even more important to me is the ability for feminist research to allow me to position my politics clearly, as articulated by Michelle Fine (1992). I have an unabashed commitment to social change in our current educational system, and see access to education as a public good and a fundamental human right, rather than a private good accessible only to the privileged few. Education is necessary not only in the narrow neoliberal conceptualizations of education as a market investment in careers that will pay well, but also as an investment in the development of each person (Tugend, 2012). Furthermore, it is important to make clear my framing of this issue as one of access, rather than a universal recommendation of college going. Not every student will want or need to attend higher education. However, the current economic and social organization of higher education means that access, while broadening, is far from universal and instead reflects (and probably deepens) growing racial and class divides (Carnevale \& Strohl, 2010; Haveman \& Smeeding, 2006). The first-generation college student may 
lack the power that comes from a family background that is attuned to the implicit rules of the college "game," but the first-generation student is by no means powerless.

Similarly, a system in which generational patterns of education can so powerfully predict individual attainment begs closer inspection, with the commitment to shaping institutions committed to social justice and furthering goals of inclusion (Saulnier \& Swigonski, 2006).

Finally, a feminist approach to the relational worlds of first-generation students allows me to keep reflexivity at the forefront of my mind and work. As a graduate student, the heaviest intellectual and emotional work that I have done in the last five years has not come from reading or thinking or writing as much as it has been born out of the continuous struggle to negotiate and re-negotiate my sense of identity in relationship to those nearest to me as I undergo the process of becoming a postsecondary educator and social work researcher. It's been clear to me throughout the dissertation process that I cannot be an impartial observer; indeed, even reading the literature and being immersed in the deficit-laden descriptions of first-generation students rendered me as something new and different: I gained a new understanding of the ways in which I was fundamentally unprepared for graduate work, but also resisted the categorical label of a troubled or struggling student. For some approaches to research, particularly those that pursue research as science, this is a troubling admission. But the feminist embrace of reflexivity allowed for the possibility, and the richness, of research that includes the voice of the researcher as well as the participants. However, my closeness to the subject also alerted me to pay close attention to differences within groups, as Reay (2012) noted: my 
experiences cannot predict others' experiences, even when we share important aspects of identity. Reflexivity was important for considering my position in relationship to the research (as well as participants) and was highly preferable to the goal of objectivity, which I could not and would not dare feign: the lack of access to education has left indelible marks on the lives and bodies of my family members across multiple generations.

\section{Focus Group Methodology}

Historically, focus group techniques were associated with market research and opinion polling, and have only recently gained a foothold in academic research (Kamberelis \& Dimitriadis, 2005; Krueger \& Casey, 2000). Robert Merton developed focus groups for use in an academic setting and published a detailed description of the methods (Merton, Fiske, \& Kendall, 1956, as cited in Krueger \& Casey, 2000). Even though Merton was influential, focus group methods were considered to be outside the realm of social science for decades, largely due to the dominance of the positivist paradigm and an embrace of quantitative methods. Academics may have also been reluctant to employ focus group methods due to the focus of Merton's work (research conducted for the War Department on the exploration of morale in the U.S. military), or due to their use in market research designed to manipulate individuals' thoughts or feelings for profit. Since the 1980s, social work in general and feminist approaches in particular have both accepted focus groups as a means of research methodology.

Wayne (2013) described the rise of focus groups in social work research: between 1988 and 2002, focus groups enjoyed steady and increasing popularity in social work 
research (as shown in a content analysis of social work abstracts (Cohen, 2003, as cited in Wayne, 2013). Focus groups were growing in popularity most rapidly in the most recent years under investigation: $38 \%$ of the references to focus groups were found in abstracts dating from the final three years of the study (2000-2002). Like Krueger and Casey (2000), Wayne argued that focus groups are a useful method for exploring individuals' perceptions and experiences, particularly when little is known about a subject. Because there is relatively little known about the relational worlds of first-generation social work students, focus group methodology was an especially good fit. While neither of these authors attended to epistemological issues, it appears that they both take a positivist or post-positivist stance towards research, as evidenced in their conceptualization of focus groups primarily as a means of extracting data from participants, who are assumed to share some experiences based on at least one commonality, through the use of skilled but neutral moderators whose job is focused on keeping participants on task. Cautions about generalizability (Wayne, 2013) also hint at an orientation towards a stance that is at odds with the claims to politics and location that I've been writing about thus far.

However, others describe focus groups not only within politics that are at peace with feminist approaches, but within feminisms themselves. Focus groups were employed in second-wave feminism primarily as a means of building theory from lived experiences (although this was mostly limited to the lived experiences of middle class, college-educated white women) (Kamberelis \& Dimitriadis, 2005). As a feminist methodology, focus groups have the potential to de-center the researcher's authority and diminish the hierarchical nature of relationships within research (Wilkinson, 1999), 
favoring "horizontal" interactions over the "vertical" interactions associated with one-onone interviews (Kamberelis \& Dimitriadis, 2005, p. 898). Focus groups may be an especially adept method for encouraging those who are marginalized (by age, language proficiency, race, or social class) to share their experiences. Kamberelis and Dimitriadis (2005) write of the emancipatory possibilities (p. 889) of focus groups in politicallyengaged collective work, and of the broader potential for focus groups as a tool for understanding the social world in ways that resist "the Western tendency to separate thinking and feeling, thereby opening up possibilities for reimagining knowledge as distributed, relational, embodied..." (p. 892). For these reasons, primarily the hope of lessening power differences inherent in research relationships and encouraging sharing among students from groups that are underrepresented in higher education, I chose focus groups as the primary method of data collection.

\section{Description of Context}

This study took place on the campus of a large, urban university in the Northwestern United States. The total enrollment for the campus was 29,452 students (23,489 undergraduates and 5,963 graduate students) (University Communications, 2014). The majority of students identified as white (62\%), $9 \%$ of students identified as Asian, $7 \%$ as Latino/a, 3\% as African-American, and $1 \%$ identified as Native American. Ten percent of students identified their racial background as "other" and eight percent are international students, most of whom were considered students of color.

The School of Social Work (SSW) is one of eight schools and colleges, and houses two undergraduate programs, a masters-level social work program, and a 
doctoral-level social work program. The Bachelor's in Social Work (BSW) program, most recently accredited in 2011 by the Council on Social Work Education, prepares undergraduate students for an entry-level generalist position in social work with individuals, families, groups, or communities. Students typically complete the BSW program in two years, taking classes in a cohort model. Sixty students are admitted annually, and 120 are enrolled at any time (M. Penners, personal communication, August 29, 2014). Among the 53 students reporting data admitted in 2014, the majority were female $(77 \%)$ and white $(66 \%)$, similar to bachelor's level social work programs across the nation. Students of color made up smaller proportions of this group: Latino/a 15\%, Asian-American 9\%, African-American 6\%, and Native American 2\%. Thirty-nine of these students identified as "first-generation" (M. Penners, personal communication, August 29, 2014).

The Child and Family Studies (CFS) program offers another avenue for undergraduate students, combining the fundamental aspects of a liberal arts degree with professional education. Students build a foundation in core courses designed to integrate theory, research, and practice and select at least one specialization (early childhood education, elementary education, special education, early intervention/early childhood special education, youth work, international work, family life education, human development/child life, families in society, child welfare/human services, or program administration), in addition to completing a practicum in a community-based setting. CFS students are prepared for entry-level positions in education or social services, or for graduate-level work in education, social work, or other helping professions. Ninety 
students are admitted to the CFS program annually, and more than 200 are enrolled at any time (M. Penners, personal communication, August 29, 2014). Among students admitted during 2014 who reported demographic data $(n=68), 47 \%$ were white/non-Hispanic, $10 \%$ were Hispanic, $6 \%$ were Asian/Pacific Islander, 3\% were Native American, and 2\% were Black/non-Hispanic. Females comprise the majority of CFS students, with 12 males currently enrolled.

Students in the Master's in Social Work (MSW) program have several options for degree completion, including two, three, and four-year options on campus, distance learning in four sites across the state, an advanced-standing option, and an online program. In addition to core coursework, students in the CSWE-accredited program select a concentration in Direct Human Services, Community-Based Practice, or Social Services Administration and Leadership. Each year 145 students are admitted to the campus-based Master's program, which currently enrolls 226 full-time students (J. Putnam, personal communication, July 11, 2014). The majority were female (80\%) and white $(82 \%)$, with students of color representing smaller proportions than in social work programs nationwide: Latino/a 7\%, African-American 6\%, Asian 3\%, Native American $2 \%$, and $8 \%$ of students reporting a multi-racial background. Similar to MSW programs nationwide, the majority of students (54\%) were between 25 and 34 years old.

\section{Selection of Participants}

Because of the in-depth nature of the research questions and practical constraints of time and funding, I aimed for smaller focus groups, seeking no more than six participants for each group. Krueger and Casey (2000) suggested three to four focus 
groups for each participant type (in this case, BSW, CFS, and MSW first-generation students) as a point at which researchers tend to find theoretical saturation. I offered several possible times for focus groups and ended up holding three groups for BSW students, two for CFS students, and three for MSW students. Because focus groups are ideally as homogenous as possible to encourage participants to speak freely, undergraduate (BSW and CFS) and graduate (MSW) students participated in separate focus groups. A second layer of segmentation, or separation into groups, occurred in the undergraduate groups, with separate groups for BSW and CFS students. Segmentation was also intended to facilitate scheduling of focus group sessions, as students in programs have some similarity in class schedules. I offered focus groups at times when a maximum number of students were on campus, to minimize barriers to participation for as many students as possible (childcare, transportation to campus outside of peak hours on campus). For the MSW students, invitations were offered to students in all programs (including online and distance option students), but most participants were students in the on-campus program.

Participant demographics are broken down by program below and include participant-selected pseudonyms and sex as well as their descriptions of racial and class backgrounds. Notice that participants have used different terms for racial backgrounds (e.g. "white," "Caucasian," and "European"; in the findings I referred to these students as “white." Similarly, students used "Hispanic," "Latino/a," and "Mexican-American." In the findings I usually opted to use the term "Latino/a" to describe students rather than "Hispanic" because it is more inclusive, but opted for the participant's descriptions when 
RELATIONAL WORLDS OF FIRST-GEN STUDENTS

they were more specific (i.e. "Mexican-American.")). Similarly, participants identified their own class backgrounds, so there was some variety in the terms used (e.g. "working class" and "working poor" suggested differences in terms of economic resources with a general sense of an enduring identification with the working class, whereas "low income" suggested temporary identification with members of the working class or those experiencing poverty).

Participants were invited to note other aspects of identity that felt relevant and where these seemed appropriate they are noted in the findings (e.g. sexual orientation in a discussion of family resistance to a same-sex relationship). 
Table 1.

\section{Participant demographics}

CFS students $n=8$

\begin{tabular}{llll}
\hline pseudonym & sex & race & class \\
\hline first group $n=4$ & female & white & working class \\
Amber & male & Caucasian & low income \\
Davis & female & Hispanic & working class \\
Lizette & female & white & working class \\
Tara & & & \\
\hline second group $n=4$ & female & white & lower-middle class \\
Amy & male & Latino & working class \\
Bob & female & Black (Jamaican- & working class \\
Brandi & & American) & "lower class" \\
Lauren & female & white &
\end{tabular}

BSW students $n=5$

\begin{tabular}{|c|c|c|c|}
\hline pseudonym & $\operatorname{sex}$ & race & class \\
\hline \multicolumn{4}{|c|}{ first group $n=2$} \\
\hline Jayne & female & European & lower-middle class \\
\hline Veronica & female & Hispanic & N/A \\
\hline \multicolumn{4}{|c|}{ second group $n=2$} \\
\hline Arturo & male & Hispanic/Latino & working class \\
\hline Juli & female & white & lower-middle class \\
\hline \multicolumn{4}{|c|}{ third group $n=1$} \\
\hline Maria & female & Latina & lower class \\
\hline
\end{tabular}


RELATIONAL WORLDS OF FIRST-GEN STUDENTS

Table 1. (continued)

Participant demographics

MSW students $n=6$

\begin{tabular}{|c|c|c|c|}
\hline pseudonym & sex & race & class \\
\hline \multicolumn{4}{|c|}{ first group $n=3$} \\
\hline Clara & female & Caucasian & lower-middle class \\
\hline Michelle & female & white & working class \\
\hline Nancy & female & white & working class \\
\hline \multicolumn{4}{|c|}{ second group $n=1$} \\
\hline Dave & male & white & working class \\
\hline \multicolumn{4}{|c|}{ third group $n=2$} \\
\hline Jaclyn & female & Native American and white & working poor \\
\hline Lainey & female & East Asian & $\begin{array}{l}\text { working to middle } \\
\text { class }\end{array}$ \\
\hline
\end{tabular}


Due to the size of the programs, some participants knew each other. Wayne (2013) emphasized the benefits and drawbacks to conducting groups with participants who know one another: participants may enjoy richer conversation and help one another elicit more memories or shared experiences, but conversations may be inhibited by familiar patterns of interaction or silencing. This was evident in some groups, as some students already had expectations about who would "go first" in speaking, for example. However, it was more common for students to be vaguely familiar with each other and "warm up" in the group conversations as they discovered shared experiences.

In addition, Krueger and Casey (2000) urged researchers to attend to gender in groups, as men may feel more comfortable speaking in groups and assume authority more easily. Brown and Gilligan (1992) write about the silencing common among women, who sacrifice voice to avoid conflict and maintain relationships. Given the smaller proportions of men in all three programs, I anticipated attending to the inclusion of men's voices, rather than the exclusion of women's. In most groups this was the case; male speakers seemed no more likely to dominate the conversation than female speakers. However, in one conversation typical patterns of gendered conversation emerged: the (older) male speaker responded to questions first and spoke freely, spelling out his ideas thoroughly before inviting his (younger) female conversation partner to speak. This conversation in particular highlighted the delicate dance between privilege and oppression: did an older man of color silence a younger white woman? Or was this woman, who noted her racial privilege, creating space for his clear narratives of experiences of racism? In this conversation I took care to invite the quieter speaker's 
voice in by using her name, turning towards her when there was a pause, and asking her if she'd felt similarly or different than her partner.

Additionally, it felt important to challenge binary ideas about gender. I anticipated creating space for students who identified as gender non-conforming or transgender. However, while facilitating the groups and conducting the analysis it became clear that the essentialist notions about masculinity in conversation were largely irrelevant in these conversations. Male participants (excepting the instance described above) spoke softly, attended to other participants' voices in the conversation, and in general seemed no more likely to dominate conversations than women. Wayne (2013) noted that ideally, all participants are invited to share their experiences and have a part in the discussion; equal time is not necessary for this to occur. Overall, students were attentive to each other's voices. Even in the conversation above when male privilege in speaking freely and feminine self-silencing seemed so prominent, both participants turned to each other multiple times, inviting the other into conversation, or asking about similar or different experiences.

Because this study was designed to understand the conditions of relational life for first-generation students in a school of social work rather than make inferences about an entire population, purposive or snowball sampling was used. In order for participants to be eligible for inclusion in focus group discussions participants had to be 1) enrolled in an undergraduate (BSW or CFS) or graduate (MSW) program in the SSW, and 2) have parent(s) and/or primary caregiver(s) who did not complete a four-year degree. Participants were informed of the study through posting of flyers, email, and in-class 
announcements, which included tentative schedules for focus groups and a brief overview of the focus of the study. Forty participants contacted me via email or in person to share their interest in participation and 19 were able to take part in focus group discussions. Due to last-minute cancellations and no-shows, two students participated in an interview when other students did not arrive at the scheduled time for a focus group discussion.

Because data collection occurred through group discussion, the process of consent looked differently than it might for individual interviews: I emphasized my inability to promise confidentiality. Participants' confidentiality was maximized through several layers of planning: 1) recruitment methods highlighted the group nature of data collection (What are your stories of navigating relationships with family or friends and school as a first-generation student? Which stories would you like to share with other firstgeneration students?), to encourage participants to consider what they felt comfortable sharing with others; 2) students who responded to flyers, emails, or in-class announcements were reminded of the limits of confidentiality; 3) prior to initiating group discussions, I reminded participants of my commitment to keeping their identities confidential through the use of pseudonyms (see number 4), and asked them to respect others' stories by doing the same. Given the group nature of discussions, however, participants were reminded to limit sharing to only those experiences they would feel comfortable sharing with others; as well as 4) participants selected pseudonyms, which were used to refer to one another in conversation during group discussions, and 5) participant demographics were attached to selected pseudonyms. Data linking participant names and their pseudonyms were kept in a locked cabinet in the SSW. For further 
details on the consent process, including the voluntary nature of participation, participants' rights to withdraw, potential risks and benefits, and study procedures, see the interview guide (Appendix D), which included a verbal reminder of these topics. The appendices also include separate consent forms for interviews and focus group discussions; I'd anticipated some students would prefer individual interviews and could provide informal feedback on the interview schedule early in the process. However, all students who responded preferred participation in focus groups rather than individual interviews.

In recognition of the time required to participate in the focus group - 90 minutes maximum, in addition to travel time (and the harder-to-quantify-but-equally-important time away from outside employment, family, homework, and self-care) - participants were given a $\$ 10$ cash incentive for participation in focus group discussions and meals were provided. All participants were invited to attend follow-up meetings in the spring of 2016 to discuss study findings (see section on trust/validity); nine out of 19 participants attended these meetings and were provided meals, but due to financial constraints, monetary incentives were not provided.

\section{Data Collection}

Because very little is known about first-generation students in schools of social work and their experiences, initial focus groups were conducted in a low-structured format (D. Morgan, personal communication, September 11, 2011). The general interview guide followed a basic funnel format, with broad opening questions to invite participation, several key questions, and a question at the end to help participants find 
closure and ensure they were able to share what they intended. I anticipated that the interview guide would change slightly during the process of conducting focus groups; some questions and themes could quickly feel "saturated." Some themes quickly emerged as common experiences, but no themes seemed to reach "over-saturation," which suggests repetition to the point of tedium. While no students opted for individual interviews to pilot the interview schedule, the semi-structured nature of the focus group conversations allowed me flexibility in providing prompts for questions that struck participants as difficult or unclear, and in subsequent conversations I felt prepared to explain difficult questions more clearly.

Opening questions were designed to set the tone for discussion and remind participants of their commonality (D. Morgan, personal communication, September11, 2011): they were all the first in their family to complete a bachelor's degree and may share some of the characteristics of first-generation students. I used an opening question that allowed participants to quickly connect and invite everyone's voice is a round-robin response to the question "How meaningful is your status as a first-generation when you're at school, or in your field placement or practicum setting?" This question sequence was adapted from D. Morgan's (2011) “Recent Widows” very low structure interview guide (personal communication, September 11, 2011). Participants were provided paper and given a few minutes to jot down responses.

This opening question tended to move naturally into conversations about the salience of participants' identities as student in their families; it was often easier for participants to speak about their student identity in family relationships than to speak 
about what it meant to be "first-generation" in school. Key questions probed participants" perceptions of their identity in familiar relationships, issues of separation and/or remaining connected, and navigating possible distances between school and home, as well as the implications for socialization into the role of professional helpers. Questions included How meaningful is your role or identity as a student when you are with your family, friends, or in your community? What significance does your role as "student" have in these familiar relationships, if any? Because the ideas of both separation and remaining connected are so prominent in the literature describing entry into college in general and first-generation students in particular, I explored these directions with participants, asking them to describe their experiences, moments, or points of separation from family that seemed to be related to their role as a student (if any). I also asked how they stay connected to family, and how they translate the experiences of school for your family, friends, or community. Guiffrida (2006) wrote about cultural distance and cultural enclaves, and London $(1989,1992,1996)$ has written about navigating social and cultural distances, leading to my third key question: How would students describe navigating the cultural distances between home and school? Where do they find spaces that provide community in school, if any? Who is in those spaces, and what do they do? Finally, I noted London's (1989) testimony to the power of family voices: How do these family voices inform your ideas about what it means to be a social worker, educator, youth worker, or other member of the helping professions?

Important in these questions was some consideration of the ways that aspects of participants' identities might shape these experiences. As students in a school of social 
work, most of the students had some classroom experience "locating" themselves in terms of race, class, gender, sexual orientation, and other aspects of identity. However, students' experiences of racism in schools of social work (Daniel, 2007; Davis, 2004) and students' possible lack of class consciousness and language to engage in conversations about class (Stuber, 2011), as well as the general lack of understanding about social class (Liu, 2011), prompted me to be ready to discuss oppressions (which would not be limited to racism and classism, but also include sexism, heterocentrism, and ageism) and the ways these play out in lived experiences. In each discussion I probed for differences in experiences of oppression and privilege by asking How do you think aspects of your identity (age, class, color, culture, ethnicity, gender, gender identity and expression, immigration status, race, religion, sex, and/or sexual orientation) have impacted your experiences as the first in your family to attend school? In teaching I have found students are quick to identify ways in which they feel oppression (women identify sexism, for example) but not as quick to recognize privileges (those same women are less likely to acknowledge how their color or race might have afforded them access or assistance in their path to school). I'd prepared to share my own (or "a friend's") experiences with classism, or to share an experience where I'd realized how my race, sexual orientation, first language, or presumed class background was operating as a privilege. This wasn't usually necessary in focus group conversations. White students tended to note white privilege, for example. But students also spoke about how gender, culture, or class shaped their experiences without specifically naming sexism, racism, or classism in their experiences with tensions with family about their educational and professional choices. 
The bulk of discussion time (60 to 75 minutes) was spent on key questions, and I prepared blocked quotes from the literature review on first-generation students' experiences (for example, the quote from Collier and Morgan's (2008) piece about the “different class of people" in college, and the quote from Lowery-Hart and Pacheco's (2011) focus groups (“college ain't me”)) to share if needed. I used these quotes in a few groups, but many conversations didn't require any extra material to stimulate conversation. Some researchers suggest also providing materials for participants to read beforehand (Krueger \& Casey, 2000). Given my knowledge of the already busy schedules of many students in general (gleaned from the literature as well as teaching experience), I opted to forego requesting participants to read prior to group discussions, but offered to share materials with participants who wished to remain in contact via email. All nineteen participants shared their email addresses in consent forms, giving permission for continuing contact about member checking and an announcement of the final dissertation defense.

Closing questions and ending a focus group require careful planning, as they provide the last opportunity for participants to ensure they've been heard and offer a sense of closure. I used the question "What advice would you give to other firstgeneration students, in thinking about navigating their relationships with family and at school?" to signal that our conversation was ending, after using cues (e.g. "I have just one more big question and one small question.”). Closing questions also provided important functions for the researcher; I used this ensure I had understood participants' stories as close as possible to their intended meanings (Krueger \& Casey, 2000). Usually 
this involved some reflection upon the broad themes of the discussion and a quick restatement of those themes. Krueger and Casey (2000) recommend allotting at least 10 minutes for the final questioning process, and I used prompts such as "have we missed anything?" Participants occasionally offered their summaries of themes (e.g. "It seems there's a lot of distancing,"), but in general they tended to offer that they were still figuring out what it meant to be a first-generation student. In retrospect asking about advice may have been difficult for participants; many participants lamented their own need for advice. These responses spoke to the potential isolation of first-generation students.

Wayne (2013) wrote about group processes that unfold during data collection in focus groups, drawing on classic ideas about patterns of group dynamics drawn from Tuckman (1965; as cited in Wayne, 2013). According to these authors, groups tend to move through a similar process of "forming, storming, norming, performing, and adjourning." Initially, as they "form," group members need to feel comfortable sharing with each other, and figure out the "rules" of interaction that will be particular to that unique group. Wayne (2013) recommended that facilitators focus on establishing an environment that is welcoming and comfortable, which included preparation of supplies (recording materials, paper and markers for name tents) ahead of time to allow me to be available to welcome participants as they arrived and engage them in informal conversation prior to groups. A key part of this involved opening statements, which reminded participants of the overall goals of the discussion and invited diverse 
perspectives. In a region known for its "politeness," 14 it can be wise to explicitly invite disagreement and emphasize the value of different perspectives on a topic that little to nothing is known about.

The second step, "storming," involved participants testing interactions, and Wayne (2013) cautioned about power struggles or participants who hold "extreme views" (p. 276). Facilitators can remind participants it's okay to disagree respectfully, reiterate the value of all experiences, and ask for other perspectives. In general this was not an issue for conversation; participants felt very comfortable saying "It's different for me," or even anticipating diverse perspectives, "For [my conversation partner] it's probably different." Quickly following this is "norming," during which participants may begin to develop a sense of closeness through a mutual understanding of the "rules" and possibly roles taken during conversation. Wayne (2013) noted that participants might begin to feel comfortable sharing personal information at this point, and the facilitator can begin shifting conversation towards key questions, continuing to support individual perspectives to avoid conformity. In most groups students quickly learned a pattern of conversational turn taking and students were quick to empathize with each other's sharing of sadness, frustration, fear, and exhaustion. For example, students matched emotional tones in conversation, responding to another's sadness with a soft voice, or expressing sympathy for a participant explaining how much more difficult it was to be a first-generation student and a parent at the same time, for example.

\footnotetext{
${ }^{14}$ For more on this see Communities of Color in Multnomah County: An Unsettling Profile, specifically the discussion on the image of Multnomah County as a bastion of progressive values (Curry-Stevens, CrossHemmer, \& Coalition of Communities of Color, 2010).
} 
Wayne (2013) claimed that the fourth stage, "performing," is characterized by more "rational" thought than the more "emotional" stage that precedes it, and is an ideal time for the facilitator to keep the focus on key questions. Participants may be better at performing problem-solving activities during this time, and this is also a good time to have alternative questions/problem posing formats ready to engage participants differently. In general, I had little need for alternative activities or prepared quotes. While I used these in a handful of focus groups, most conversations presented no lull where quotes might have been fitting to stimulate further discussion. Several participants noted they were still figuring out what it meant to be a first-generation student, which seemed relevant to the finding that many first-generation students lack a sense of group identity: students were surprised to discover their experiences that were often difficult to describe were not theirs alone. Finally, as discussed above, "adjourning" is an important time for facilitators to be mindful of the different needs of participants. Participants may be ambivalent about wrapping up discussion: some may be eager to finish, others may loathe ending a discussion that allowed them to connect to others with similar experiences (Wayne, 2013). Following the focus group discussions participants often stayed in the room, and we continued talking after I'd turned off the recorder.

The role of the facilitator (often called moderator) receives a lot of attention in discussions about focus groups, but Morgan (personal communication, September 10, 2011) noted that in terms of moderation, the less done, the better. In general, participants want a discussion to flow well, and will often work to help out a moderator who seems to be in trouble (D. Morgan, personal communication, September 10, 2011). However, it 
would be a mistake to think that facilitation doesn't require any skills. Wayne (2013) wrote that facilitators should be "lively, likable, interested, engaged, flexible, perceptive, and analytical" (p. 277), in order to meet their goals of engaging participants in discussion. The facilitator should be a good oral communicator, able to insert questions and probe when answers are unclear, while still maintaining a minimal role in the conversation. Similar to guiding discussions among students in a classroom, the facilitator should be able to quickly paraphrase responses and narrate a storyline for the conversation, to keep participants engaged and understanding one another's thoughts and ideas as the conversation unfolds. I found myself reflecting on and growing in these skills throughout the process. My initial focus group presented a challenge as my novice was compounded by the pre-existing teacher-student dynamic: three of the four participants have been my students in classes. Fortunately I realized they were responding to me in a question-and-answer format early in the conversation and was able to invite them to shift their focus to each other. In subsequent groups I practiced limiting my voice while also remaining engaged in the conversation and guiding its direction.

Morgan (personal communication, September 10, 2011) emphasized that the goal is not necessarily equal sharing among participants: some people tend to talk more or less than others. The facilitator needs to be attentive to nonverbal communication: aware of body language and skilled in using gestures (eye contact, hand gestures) that invite participation while remaining rooted in a role that is primarily about listening, not being heard. Gestures might be particularly useful when certain members tend to dominate a conversation; eye contact or a movement toward a quiet participant can offer an avenue 
into the discussion, as many people will want to be heard (D. Morgan, personal communication, September 11, 2011). Wayne (2013) also noted that facilitators should be sensitive to group dynamics and have a good sense of timing, as enough time needs to be spent on key questions, and tasks like closing questions serve an important role in the process. One of the most critical skills for the facilitator is comfort with silence during discussions (D. Morgan, personal communication, September 10, 2011; Wayne, 2013). David Morgan, a researcher with years of focus group practice, noted that not pausing long enough after asking questions is the biggest mistake facilitators make; facilitators should be ready to endure silence and invite conversation by (silently) counting to ten after posing a question. Again, this skill echoed my experiences in classroom discussions: many students need a moment to compose a response they feel comfortable sharing with others and it's important to resist filling in the gap as an instructor, therefore setting a tone that silences are to be feared. I found that silences were extremely rare in these discussions. In general, students were eager to share their experiences and tended to make space for each other to share as well.

Focus group sessions were audiotaped using a digital recording device and transcribed as soon as possible afterward. Three focus groups were transcribed during spring 2015, allowing for preliminary reflection on analysis and participants' responses to the interview schedule. I attempted to videotape focus groups discussions to capture significant pauses, expressions, moments of shared laughter, and non-verbal messages. However, after the first two groups it became clear that video recording was not possible and I focused on noting significant non-verbal messages in the spoken conversation (for 
example, describing the "net" gesture Bob made with his hands and asking him to elaborate on my understanding of that in the recording so it would not be lost in analysis). Following transcription of initial focus groups, I printed hard copies to become familiar with the physical volume of data, as my previous work was limited to transcripts from individual interviews, the longest of which was 25 single-spaced pages. Transcripts from focus groups vary, but can be between 30 to 50 pages (Krueger \& Casey, 2000). I found that analysis was possible, and even preferable, using hard copies of transcripts for the multiple readings of transcripts and analysis, described below.

\section{Data Analysis}

Conversations in focus groups prompt new insights or deeper reflection among participants than single interviews, providing for richer and deeper responses, but this depth can lead to an overwhelming amount of data (Frankland \& Bloor, 1999).

Balancing the amount of data and the richness and depth with the need for systematic analysis was important. Analytical procedures for focus groups vary from the brief (a written summary by the facilitator immediately following the discussion) to the careful analysis of each transcript. Due to the exploratory purpose of this research and the fact that little is known about the conditions of relational life for first-generation students in schools of social work, each transcript was analyzed systematically, as described below.

While preparing this section of the proposal, I became aware of the danger Wilkinson (1999) noted of potentially “insufficient epistemological warranting” (p.77) for interpretation of data. Texts that guide qualitative researchers often present methods of analysis divorced from considerations of epistemology, and the implications for 
interpretation are important: should data be understood as essentialized views of an experience, or from a constructivist position wherein experience is part of a coconstructed reality? Fortunately, while many descriptions of analysis left questions of epistemology untouched, it was often hinted at. For example, Marshall and Rossman (1999) described the basic steps of qualitative analysis as 1) organizing the data, 2) generating categories, themes, and patterns, 3) coding the data, 4) testing emergent understandings, 5) searching for alternative explanations, and 6) writing the report. At least two of these steps suggest a post-positivist framework oriented towards the ability to describe, and if not infer, at least predict or explain. In this section I'll cover steps the steps of analysis that I'd planned for this project and weave in my descriptions of what I actually did during analysis. Due to how little is known about the topic and the aforementioned location of this work in a postmodern/post structural feminist framework, I planned for and carried out three layers of analysis: analysis of single transcripts, analysis of transcripts across groups, and writing as analysis.

\section{Analysis of Single Transcripts}

Following transcription of each focus group discussion, I read the transcripts multiple times using a voice-centered relational method of data analysis (Brown \& Gilligan, 1992). This method was designed for single-person interviews, and I was concerned about using it with focus group discussions. But it offered possibilities not only for exploration of participants' relationships but also for making plain the relationship of the researcher to the process of research; I felt this was important given my epistemological grounding and relationship to these questions. I focused primarily on 
the exchanges in conversation, but also on individual images and stories that emerged during groups in which a student was especially specific, enthusiastic, or passionate; these are Krueger and Casey's (2000) markers for identifying meaningful data during data reduction. Many of the critical meanings and moments in the conversations happened between two, three, or four participants, and therefore I could focus on a part of a conversation in analysis rather than the individual story that Brown and Gilligan (1992) used as their focal point in the original description of voice-centered relational data analysis. At other times one person's story seemed of central importance, and then I would focus on that individual as described in other uses of voice-centered relational data analysis. As I proceeded through the four readings (described below) I came to imagine the focus group discussions as braids formed with single strands contributed by each participant. At some points the participants' words ran together closely as they shared similar experiences and feelings; here the braid was tight and I could focus my analysis on these exchanges. At other points participants' strands were loosely bound together as they took turns describing different perceptions of an experience. In these cases the analysis looked more like Brown and Gilligan’s (1992) intended use.

Regardless of whether my focus was shifting from the exchanges to the individuals, I used the same listening guide (see Appendix E) to focus my attention on different aspects of the conversation during each of the four readings of the transcripts. During each reading of the transcript I used corresponding highlighters to note key parts of the transcripts relevant to each reading. I jotted down brief memos in the margins of the transcripts and color-coded these by reading as well. After each reading I expanded 
these memos in a longer written description of my analysis of each reading; these were typically two to seven single-spaced pages for each of the four readings. Where appropriate I included excerpts from transcripts to clarify my observations and reflections. Using four readings and writing-intensive analysis allowed me to become intimately familiar with each conversation and made it easier to write when I began to write the findings and discussion.

During the first reading, the focus is on the story and who is speaking: the researcher records their responses to the speaker and attempts to tell the overall story the participant has shared (Brown \& Gilligan, 1992). Because these were group discussions rather than single interviews, I anticipated challenges with sketching a single storyline. But I found that often even a single speaker didn't necessarily have a clear storyline, and the prompts and questions in this first reading allowed me to note these points of confusion or misunderstanding about the coherence of their narratives. In this first reading I noted themes that were shared between the participants, using Morgan's (personal communication, September 11, 2011) advice about analysis within groups: what are the topics that everyone wants to discuss? They may not all see it the same way, but which themes emerge as something that participants feel passionate about? In this first reading, I noticed that certain phrases and images tended to emerge for students. Several students used familiar refrains (often a family saying about their education) or evoked a role or image ("the poster child" or "hero," for example) in their conversations. I noted these phrases, images, and roles as key pieces of the "plot" or "storyline" for students' descriptions of their relational worlds. 
One of the most useful parts of the listening guide was the attention Brown and Gilligan (1992) gave to relationship as a necessary prerequisite for voice (after all, we don't need to vocalize thoughts that we keep to ourselves). This informed a final piece of the first reading, reflecting on my own relationships, however brief, to the speakers in these conversations: how did I identify with the speakers, or not? How did I feel about their story? I found that a few speakers were intimidating to me as an emerging focus group facilitator. A few students quickly assumed roles directing the conversation, and I found myself striving to connect with these students on points of assumed similarity or working to ensure that they knew I heard their stories. Brown and Gilligan's (1992) first reading provided me a framework to consider how my own feelings were driving data collection and analysis, and record my own reactions to these stories. Sometimes I was surprised by how different participants' experiences were from my own, and often I felt deeply moved by these students' articulations of feeling different or separated from important people in their home cultures.

The second reading involved a focus on participants' sense of self. In keeping with Brown and Gilligan's (1992) focus, I highlighted all of the instances of "I," "me," or "my" and paid close attention to those sentences. In essence, this reading is focused on noting how the participants describe themselves. I found it was sometimes difficult to tease out descriptions of self from descriptions of self in relationship. For obvious reasons, this reading was the one where I focused primarily on speakers as individuals, and it felt the best suited to these methods, which were developed for interviews. However, I did notice the Western cultural bias in Brown and Gilligan's (1992) assertion 
that speakers would naturally construct a sense of identity in referring to themselves in a singular "I," "me," or "myself." Students of color were more likely to speak of themselves as members of a community of people with a shared heritage. I noticed in one student's transcript in particular during the second listening that I needed to highlight "we," and "us" to get a sense of his identity because he was much more likely to speak about himself as a member of a group than as an individual. In this second reading I noted participants' descriptions of who they were: important parts of their identities, feelings and emotions, and key images ("the family star," or "the smart one," for example).

These first two readings are designed to establish a sense of relationship with the speaker. Brown and Gilligan (1992) noted the importance of listening to how a speaker constructs herself or himself before she or he is "constructed" by the researcher. As a researcher I appreciated this attention to listening to the speaker first (even given the cultural assumptions embedded in this approach that I've mentioned above). I've often felt reluctant to describe research participants with the air of authority that's a presumed part of the role of a researcher and the specific focus on listening to how speakers described themselves helped me feel like I knew how they wanted to be seen.

Third, the transcript is read with an eye towards relationships with others. How do participants describe interactions with others? Brown and Gilligan (1992) emphasized participants' descriptions of authentic relationships, ones in which they felt able to express themselves freely. What roles do/might oppression play in power dynamics they describe? These interactions may be with individuals, but may also reflect interactions 
with members of other groups. Fine and Weis (2005) argued for the necessity of attending to relations between social identity groups, rather than treating participants as members of social groups which exist in isolation. In the second and third readings it may be fruitful to attend not only to participants' discussion of relationships with family, community, and school, but also their interactions with other people in the room. How do participants' relational worlds unfold in focus groups, how do they construct a sense of self in relationship for others, and engage in relationships, however brief, in the focus groups?

This third reading was the most detailed and time-consuming, but the previous two readings allowed me familiarity with the transcripts so I approached the third reading anticipating areas of importance. In reading through the transcripts and highlighting key portions about relationships many of the themes of this work began to emerge: students were supported, but only to an extent, students felt a growing distance from people in their home cultures, and students were invested in maintaining these relationships and so worked to bridge these distances. Similar to the first two readings I highlighted portions of the transcripts where participants discussed their relationships and jotted down memos in the margins. As in each reading, I expanded these memos into longer observations on participants' stories about relationships. These sections of this written analysis were the longest, ranging from five up to 15 single-spaced pages. The third reading also contained the most excerpts from transcripts, as I wanted to provide clear connections for my observations to the data. 
The fourth and final reading involved reading with attention to the participants' relationships to their larger political, cultural, and social context. How do participants see their experiences fitting in to the broader society around them? Have participants developed a sense of the "sociological imagination" (Mills, 1959) that Fine and Weis (2005) noted is uncommon for many individuals, who fail to make connections between their own private troubles and the broader political, economic, and social context. I found this to be true in many instances: participants often spoke of their experiences in highly individualistic terms. Several students noted the influence of gender or culture on their family's expectations for them, and one student explicitly named institutional racism as a factor in his experiences in school. Some students noted class as something that made their experiences fitting in more difficult, and several hinted at struggles with social mobility. However, it was less common for students to connect these influences with a broader political, cultural, and social context. This reading also focused on self-silencing in relation to cultural norms and values. I suspect this is related to the observation above that students may not make clear connections to their personal experiences and the broader political context. In my writing about this fourth reading I noted when students were making connections between their experiences and the broader society. I also noted when there were political, cultural, and social implications for their experiences, even when students spoke about these as their own individual experiences.

Paying attention to not only what is said, but what is not said was critical to attend to in all readings (Brown \& Gilligan, 1992). In the proposal I noted the potential drawback of Brown and Gilligan’s (1992) description of silencing as a gendered 
phenomenon; to attribute this tendency to women's development seemed to pose the same trap of essentialism we encounter in second wave feminism's assumptions about the "universal" experience of women. As described above, gendered self-silencing in the face of sexism seemed most prominent in one focus group conversation when two participants were the least matched in terms of race, age, and sex. In most conversations women seemed no more likely to self-silence than men. I've also highlighted the drawbacks above of constructing participants' sense of self entirely in terms of the individual. But despite my hesitations, voice-centered relational methods offered one of the most compelling and clearly articulated descriptions of analysis which attended to power, difference, my relationships to the participants and the knowledge, and the qualities of relational worlds.

\section{Analysis of transcripts across groups}

An advantage of focus groups included the ability to generate rich insights through discussion, but because each group offered a new chance for different combinations of sharing and comparing, it was important to compare transcripts between groups. After completing the four readings of each of the eight transcripts and writing about them, I had a voluminous amount of data. I'd proposed using Krueger and Casey's (2000) "long-table" approach ${ }^{15}$ or Morgan's (personal communication, September 11,

\footnotetext{
${ }^{15}$ The "long table" approach is quite literal, involving the use of a long table, upon which transcripts can be cut up and separated into similar sections under broad groupings (similar to indexing). Ideally, this table should be one where transcripts can be left for a time without being disturbed, so that they can be manually re-shuffled as the analysis progresses from indexing to progressively more refined differences between quotes and stories. Since most of us don't have empty and unused long tables lying around, Krueger and Casey suggest taping transcripts to the wall could suffice, although the name "taping things to the wall" doesn't have the same ring to it.
} 
2011) "grid" analysis. These authors advise breaking down transcripts topically, in this case grouping answers to the same questions together, to gain a sense of which key themes arise in all discussions. As I'd anticipated, participants' responses varied somewhat, even in response to the same questions. While doing analysis across groups I focused on themes that emerged in multiple groups, while still attending to themes which were less frequent, but salient.

Morgan (personal communication, September 11, 2011) offered a more general strategy of induction, creating codes from the "bottom up" through reading transcripts. Because of the depth and complexity of the writing I'd produced in the four readings of each transcript, I didn't go back to the transcripts but instead read through the analysis to pull out themes. My goal at this stage was summarizing themes across groups. Here it was useful to keep Krueger and Casey's (2000) questions in mind: which themes were frequent (mentioned often) and extensive (mentioned by more than one person)? Which responses or exchanges were specific and detailed? And which responses or exchanges were especially engaging for participants? Which topics inspired emotion or arouse enthusiasm? ${ }^{16}$ I read through the analysis for each group and summarized themes on sticky notes (noting page numbers back to related quotes in the transcripts), using different colors for BSW, CFS, and MSW groups. The sticky notes allowed me to arrange and re-arrange them into common themes and aided in the overall organization of the findings section.

\footnotetext{
${ }^{16}$ See Table 2 for a diagram outlining the anticipated approach to analysis of transcripts across groups.
} 
RELATIONAL WORLDS OF FIRST-GEN STUDENTS

Table 2.

Anticipated outline for analysis of transcripts across groups

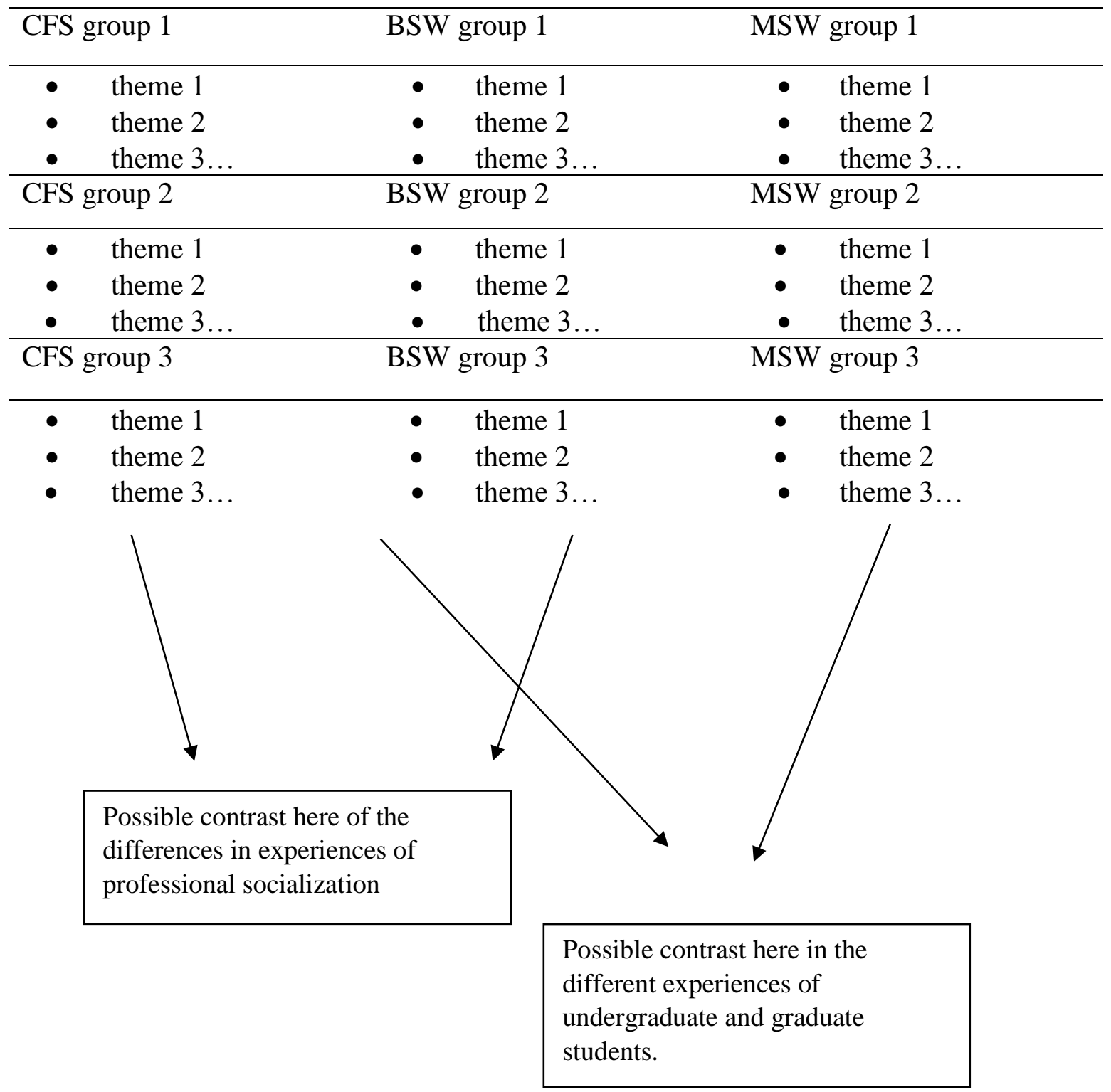


At this point a broader vision for organizing written results began to take shape.

Here I needed to attend to Wilkinson's (1999) comments about keeping conversations in context throughout the analytic process. Publications involving focus groups often inadvertently omit one of the strengths of focus groups, conversational exchanges, instead limiting quotations to blocked texts from single speakers (this was seen in Orbe's (2004) work, but not in Lowery-Hart and Pacheco's (2011), which included extensive exchanges between multiple speakers). As much as possible during analysis and writing the findings, I tried to include exchanges rather than only single speakers. I didn't want to lose the richness of these conversations.

I'd anticipated potential differences between groups, and using the color-coded sticky notes fostered comparisons between groups. I was able to tell which experiences tended to "cluster" among students in a program and see which differences I'd anticipated (such as a difference between graduate students and undergraduate students' experiences) didn't emerge. Once I had a general idea of the broad sketch of the findings I was ready to begin writing. However, as I'd emphasized in the proposal, the writing I'd been doing throughout this process aided me in the final stages of preparing this study.

\section{Writing as Analysis}

Writing up findings is often considered the final stage of analysis, but many researchers have emphasized the important role this plays throughout analysis (Marshall \& Rossman, 1999; Richardson \& St. Pierre, 2005). Richardson and St. Pierre (2005) noted how instruction in writing is often based on a conceptualization of writing as a linear, orderly process. We are taught to outline ideas first and begin writing only when 
we know what we are going to say, following a predictable format that will make our writing fit right in with the dozens, perhaps hundreds, of manuscripts that Richardson admitted to "yawning" through, often abandoning half-read. Richardson argued that these modes of writing are rooted in positivist traditions and reflect the assumptions of traditional science.

Instead Richardson and St. Pierre (2005) argued for the value of writing as a means of inquiry, and for attention to voice and creativity in the process. After all, what good is investing the amount of time and energy typically needed to produce a dissertation or published piece of research if it's too dense or dull for anyone to read it? While publications are critical for tenure and promotion, my political positioning in relationship to this research drove me to produce something that will engage readers in the institution as well as outside of it. I wanted participants to not only be able to read this work, but to want to read it. As bell hooks (1994) has observed, "any theory which cannot be shared in everyday conversation cannot be used to educate the public" (p. 64). It seems fitting that a study focused on relationships to family among those who are new to the university should at least attempt to be accessible to family and community members with limited exposure to the academy. In honoring this desire I've attempted to keep my voice present throughout the findings and discussion. Indeed, in member checks and follow-up discussions with participants, several have asked about reading this work.

I also emphasized writing as critical to analysis because it forced (or allowed) me to engage deeply with participants' language. As I read and wrote and re-read and wrote more about these students' words I became familiar with the discourses that were 
activated for them as they took on roles as students and professionals. Writing about the participants' words and ideas throughout multiple steps of analysis allowed me to engage deeply and repeatedly with the images and phrases that arose for them when describing their relational worlds. Richardson and St. Pierre (2005) also noted the ways that writing supports thinking: writing requires different movement and engages different ways of knowing than following steps of analytic induction or sifting through data in a qualitative coding program. For the researcher, writing as analysis also allows attention to subjectivity (which is often a central part of inquiry in feminist approaches to research). Ideally, writing throughout the process provides for the researcher to be located within the text, and for a retelling that is "partial, historical, and local" (Richardson \& St. Pierre, 2005, p. 962). I found that using a writing-intensive method of analysis allowed me to remain sensitive to my own location in relationship to the unfolding stories of participants' relational experiences and clarify my thoughts as I was writing.

My first draft of the findings was voluminous, breaking down the findings into rough categories which broadly outlined the flow of students' conversations about their relational worlds, moving from support to being on their own to growing distance. Following feedback on this initial draft, I've attempted to represent the findings in a graphic that provides an overview of the headings and sub-headings and provides a rough visual sketch of the distance between the cultures of home and school and the journey students make between each, as well as their experiences of working to become integrated in one and remain integrated in the other. In a final round of feedback and revisions, I've brought these findings back into conversation with some of the dominant 
theories used to frame explorations of first-generation student experiences, namely Bourdieu (1986) and Tinto's $(1975,1993)$ work.

\section{Establishing Trustworthiness}

Like most students educated in the $20^{\text {th }}$ (and early $21^{\text {st }}$ ) Century, I was trained in the processes of the scientific method and told these procedures, if followed carefully, would yield accurate, reproducible results, unblemished by whatever thoughts or values I might hold about the subject under investigation. This training was not limited to my graduate or undergraduate education, but began in elementary school; like most students, I have been steeped in the view of science as (value-free) method. The concepts of reliability and validity, embedded in the scientific method, were embraced by many who sought to apply principles from scientific study to the study of the social world. However, certain assumptions of the scientific method (such as objectivity or the assertion that there is a final, indisputable "truth" regarding social relationships that can be uncovered) remain untenable to many who engage in research with others. Lincoln and Guba (1985) argued that reliability and validity should be replaced by credibility and trustworthiness (explained in further detail below).

Seale (2002) argued that Lincoln and Guba (1985) have misread the work of empiricists and are too hasty in their rejections of positivist methods. Seale took a pragmatic approach, claiming that methods were only loosely tied to "moments" in qualitative inquiry (see Denzin and Lincoln (2005) for a further description of these moments). For example, Seale claimed that qualitative researchers who eschew the assumptions of an empiricist stance (value-free, search for a knowable truth) can employ 
the method of triangulation through examining multiple perspectives on a problem (multiple data sources) and attending to differences in those views. Seale likened triangulation to a prism in which the differences in the beams of life refracting through the crystal become the focus or the "findings."

Seale's (2002) thinking was seductive on two levels. First, he argued (correctly, I think) that there are real dangers in ignoring the consequences of methodological decisions. As I mentioned above, I was trained in a certain view of science that has established "rigor" and seems to show no signs of diminishing in its sense of power in the academy. As a former psychology student who spent time reading and considering Cook and Campbell's (1979) threats to validity and attending to these in research designs, I agree with Seale that this thinking is not superficial and, if read carefully, sensitizes one to the importance of the details of research design. Second, I was compelled by Seale's (2002) appeal to the difficulty of establishing standards for judging trustworthiness: "Quality is elusive, hard to pre-specify, but we often feel we know it when we see it" (p.102, emphasis added).

However, Seale's (2002) implication that methods are only loosely tied to "moments" (and by extension, paradigms), and can therefore be employed in pursuit of rigor regardless of one's own epistemological position was troubling. While I am aware that here I ran the risk of epistemologizing methods ${ }^{17}$ (Leotti \& Muthanna, 2014), it

\footnotetext{
${ }^{17}$ Leotti and Muthanna argue for the inclusion of quantitative methods in feminist approaches to research, highlighting the binary construction of feminist research as inherently qualitative. By assuming that qualitative methods automatically align with feminist epistemologies, or more importantly, that quantitative methods do not, we "epistemologize" methods.
} 
seems inaccurate to assert that qualitative researchers, regardless of their stance, can simply rely on methods set forth by those who embrace a realist perspective and divorce their research from the broader political contexts those issues arise within (Seale uses Hammersly's (1992) discussion of criteria to outline truth and relevance). This claim was particularly difficult for me to swallow in light of the fact that research does not occur in a vacuum, especially research focused on education (as Smith and Hodkinson (2005) illustrated in their discussion of the implications for qualitative research in education following Shavelson and Towne's (2002) critical review of qualitative research in education). Not only does research take place in a context of power and politics (think funding), but individual researchers are socially and historically located, and cannot escape their own socially situated viewpoints. We make our claims based on our social and historical location, and this seems to prompt recognition of the relativism within which we are bound. However, Smith and Hodkinson (2005) pointed out that an acknowledgement of relativism does not mean that all claims are equally valid, but "stands for nothing more or less than recognition of our human finitude" (p. 922). These authors argued for a reconstruction of research, for those who embrace this notion of our own views as limited, from "discovery and finding" to "constructing and making" (p. 921). Criteria, for these authors, involved more than a reliance on methods, but were something that is socially constructed and shifting.

With that said, in recognition of my own finite nature and the limits this imposes on my thinking, I outlined methods I employed throughout in pursuit of trustworthiness and credibility. Lincoln and Guba (1985) advocated for credibility (rather than truth), 
and suggest member checks as one avenue for assuring credibility. My previous work with foster youth and former foster youth erupted my ways of thinking about validity (was I really "measuring" what I thought I was measuring or "discovering” the findings I was looking for?). In the years since I completed this work and shared the findings, I have found the most accurate barometer of its credibility, for me, has come from hearing from former foster youth who read this work and recognized their own experiences in the stories that were shared with me.

I used member checks with the participants in the focus groups after analysis to de-brief and invite participants to evaluate both my "take" on their conversations and my interpretation of those conversations. I offered four potential sessions for member checking and nine of the original 19 participants attended. In initial sessions I shared an overview of the broad themes with as many quotes as possible. My goals were twofold: to ensure that participants felt comfortable with their words and the way they might be represented by these words, and to check that these themes resonated with participants. I quickly learned that fewer quotes were easier for participants to engage with; participants were eager to read everything I provided. In general, both goals for the member checks were met: participants felt comfortable with their words and representations, and agreed that the themes about remaining to connected to family and home cultures were true to their experiences. In truth, I had a third goal of inviting participants to experience some of the comfort I'd found in hearing from others with shared experiences, and this was a byproduct of the member checks. Participants read each other's words carefully and often commented, "Oh, someone else thinks that too? I thought I was the only one." 
Lincoln and Guba (1985) also suggested notions of reliability be replaced with dependability, and that confirmability be substituted for neutrality. One method commonly employed is the use of an audit trail, which includes the keeping of a records about decisions made through the research project. Because this project is rooted in a feminist approach which acknowledges the relationship of the researcher to the researched and subjectivity, attention to reflexivity is critical, and will be included in these records. The use of voice-centered relational data analysis incorporates some aspects of this through attention to the researcher's reaction to storytellers and their stories. I kept an audit trail via a Word document where I tracked key points and decisions in the process (e.g. IRB notes and approval, decisions about including a student in the research) throughout the project. Supporting reflexivity was mainly done through voice-centered relational data analysis: I was able to record my thoughts and feelings in the first reading in particular, but also make my voice plain throughout the other three readings.

Peer debriefing (Padgett, Mathew \& Conte, 2004) is another means of ensuring dependability and confirmability, and includes regular discussions with knowledgeable others who can offer instrumental (primarily) and emotional support in the process of conducting and interpreting qualitative research. Padgett and colleagues emphasized the importance of peers: that this de-briefing takes place among those who are engaged in horizontal relationships, such as fellow students or between researchers, rather than relationships involving a power imbalance, such as between faculty and student, or mentor and mentee. Although I appreciate the sensitivity to power, I hope to stretch the 
meaning of "peer" in this instance to include discussions with faculty who are engaged in research similar to the work I want to do, or who possess great depth and insight into critical and feminist work, or who have a keen understanding of pedagogy, or a combination of these things. Throughout this process it was difficult for me to keep in regular contact with advisors. I did a lot of dissertation work on weekends and in the evenings and in general "around the edges" of my work and family life. But I was fortunate to have a semi-regular writing time with several faculty members that aided greatly, impromptu writing and discussions with friends and colleagues, and a dissertation chair and committee members who were quick to respond to questions and always showed interest in this work.

\section{Researcher Positionality}

I agree with Sandra Harding (1993) that it is a mistake to assume we could remove our fingerprints from the work we will do, and my own relationships to schooling, family, and the shifting constructions of my identity have remained at the forefront of my thinking throughout writing this proposal. My own relational experiences as a first-generation student in a school of social work exist at the nexus of two worlds that I have occupied for the majority of my adult working life (if not longer): education and social work. However, my relationship to each is one that is lived on the margins: part of me is always in and part of me is always out, but I am never fully situated in either one. 
I have written elsewhere ${ }^{18}$ of the dialectical tensions I feel regarding education: I am deeply in love with learning, and yet profoundly disappointed by too many of my experiences with education. My early experiences of education were a complicated mix of wonder at observing the rhythms of passing seasons, listening to stories and learning the language of letters and numbers, and making friends that stayed with me through schools that became increasingly overcrowded and underfunded and lacked any systemic support for students from families for whom the transition to college was a complete mystery. When I was finally able to enter a university, twelve years after graduating from high school, I did so timidly, unsure of how I would fare in an environment that I had long imagined as enveloped in heady philosophical debates. After recovering from the initial shock that my classmates didn't all resemble the pipe-smoking, poetryspouting ${ }^{19}$ students in the movies that had somehow shaped my ideas about the intellectual rigor of the university, I was overwhelmed with the feeling of wanting to invite my family to follow me into this world. I imagined how my grandfather, who spent his evenings poring over one of the library books from the stack that always sat by his chair, or my cousin, with whom I had been debating politics since we were 11 and 12, or my niece who, quickly and almost effortlessly, cracked the "code" of reading, would enjoy the same opportunities I had for exploration and debate in the classroom. As the first one in my family to attend a university, I wondered how their lives would have been, or could be, different if they were afforded the same opportunity.

\footnotetext{
${ }^{18}$ This continues to be part of my written teaching philosophy.

${ }^{19}$ This wouldn't have necessarily been a bad thing... but clearly suggests how vague and foreign the world of the university seemed.
} 
My grandfather lost interest in reading those books when he was "let go" from his supervisor position in the paper mill after more than twenty years of work, due to his eighth grade education. He lived out the rest of his life in relative poverty. My cousin's and niece's stories are still unfolding, and out of respect for them I cannot put their struggles into words, but it is their faces that arise most clearly in my mind when I say that a lack of access to education has marked the lives of those I love, and in many cases, their bodies, with the indelible effects of poverty and limits to both vocational aspirations and learning itself that are imposed when the process of education is deadening, dull, and dehumanizing. Perhaps if they were able to sit in classrooms, to experience the same excitement I felt when going through the steps of writing: reading and thinking and debating and poring over possible combinations of words until I found the ones that felt just right, then their stories would turn out differently from our grandfather's.

However, my first reactions of joy began to dissipate in graduate school, as I came to realize the changes in my own thinking and language that seemed to negate the ways of being that I had learned from my family. The words I used, and the types of knowledge I would accept as "legitimate" as someone developing a new identity as a "researcher" were out of step with the ways of thinking and knowing that were part of my upbringing. I wondered if inviting family members might bring them the same pain, and begin to (quietly) wonder whether it was better to attend school at all if it meant feeling torn between the identity I thought I should develop as an academic and the identity that I had developed over thirty years through my relationships with family and friends. 
bell hooks' (1994) work saved me from the sense of being completely alone, writing of the "psychic turmoil" that students from "class backgrounds that are deemed undesirable" (p. 182) sometimes feel in the classroom and pointing me towards Ryan and Sackrey's (1996) work, where I recognized the potential stories of my grandfather in the tales of these mostly white, male academics who benefited from the democratization of the academy that took place in the years following World War Two. I couldn't resist the urge to wonder how my life and my family members' lives would have been different if my grandfather's story had been one of theirs. The academic life, Ryan and Sackrey argued, is anathema to the working class way of life. I had some sense of this in the difficulty my family members (and I) had in seeing what I was doing as "work," sitting in front of the computer for hours on end. While I enjoyed a level of autonomy I could never have imagined in my previous work, I felt constrained by the blurring of work and home life, and negotiating this boundary while "remaining intelligible" (Gergen, 2009, p. 140) in relationships with family and community was often harder than the actual work I was doing.

At this point, it's important to pause and note the difficulties I have with the concept of "class" in general. Any sense of myself as being not "middle class" did not emerge for me until I went through a graduate program in education and, during the process of professional socialization as a "teacher," ${ }^{20}$ realized how closely standards of professionalism aligned with middle-class standards for interpersonal interactions, and in particular dress and appearance. It was through listening to my middle-class peers impart

\footnotetext{
${ }^{20}$ A term which I resisted, preferring "educator," because too much of "teaching" in my master's program seemed to involve replicating modes of instruction which hadn't served me very well as a student.
} 
their own experiences (vacations in Europe, the car their parents had purchased for them ${ }^{21}$ ) that I begin to slowly question my own assumed location in the middle class. I was troubled by how much I had to dwell on my ability to afford the right clothes, as someone moving into the role of a "teacher," and the gendered and classed implications of which students were marked as "professional" by some instructors in our program did not escape my notice. No matter how well I taught, I was unsure that I could achieve the role of "professional," based on my limited income.

But appearance and material possessions were superficial, and didn't even begin to touch the difficulties I felt with adhering to standards of interpersonal interactions which guided "small talk" and seemed to be entirely constructed in a view of people as potential capital in a social network. I also came to see the complications in my family's view of education: as something to be valued and pursued to a point, but not something that was assumed after high school, unless its pursuit didn't interfere with work. I was an educator in love with learning who clearly felt that I couldn't fill the role. I found in hooks' (1994) and Ryan and Sackrey's (1996) work a "home” in an identity as a working class person. However, I also realize the shifting and incomplete nature of this status: although these authors claim working-class backgrounds as tenured professors, I also notice the relative privileges afforded in doctoral study, and question how much I can claim this background as a doctoral student, or will be able to claim it as an instructor. I've embraced the values of a relational worldview which was part of my upbringing, and

\footnotetext{
${ }^{21}$ By no means were these experiences shared by all students, but they provided the sparks of a growing class consciousness: realizing the ambiguity of the term "middle class" to describe a social group and the many ways in which my family was not middle class.
} 
have recognized in countless interactions how out of step these are with some modes of interaction in the middle-class halls of the university, but I also think it's simplistic to assume that this means that my relationships are fundamentally different from the relationships middle class students or instructors have with their families, or the value they put on relationships. And perhaps most importantly, I have embraced the middleclass expectations for higher education for my own children and family, or at least the need to support access to higher education.

I see my family's instruction in my own dedication to work, and I'm proud we were taught to value work, but I want my children to know that their paid labor should not overshadow their ability to get an education. Even though I embrace a multi-layered conception of my own class background, I have certainly felt the "psychic turmoil" that hooks (1994) speaks of. However, I agree with hooks that the joys of learning are great enough that I should work to remain, even if it means that I am always on the margins: an "educator," but not a "teacher," a "student," but not one who fits in the traditional mold assumed in the university.

Similarly, my relationships to social work are complicated by insider-outsider tensions. The majority of my working life was spent working as an educator alongside social workers in a state-funded preschool program serving children and families in poverty. I participated in needs assessments and grew comfortable and familiar connecting families to outside agencies to help with their health, housing, employment, and educational needs. At its best, I worked alongside social workers in a capacity that allowed families to highlight their strengths and build relationships with teachers in the 
public school system, as well as feel competent in meeting their family's needs amidst experiences of poverty. At its worst, I realized that these families' readings of me underscored the transgressions of social work. More than once I arrived at a family's home to meet a child who refused to step out from behind their caregiver's body when I entered the room, and was later told that someone who looked like me (a white woman who appeared to be a middle class professional) had judged their family as deficient and removed the children. For these families, my role as an educator eventually became more prominent, but social work, and the history of "friendly visiting" was something that I carried with me into every interaction.

While I've recognized my roles as intertwined with social work, I am not a social worker. Indeed, I have spent a more time as a client ${ }^{22}$ of social work, representing my family or me. Disability, death and dying, and experiences of poverty and near poverty that are so common among many Americans (Rank, 2013) have placed me in the seat opposite a social worker far more often than in the seat of the professional helper. I wrote my proposal scribbling on legal pads I balanced on my lap while wedged in between strangers in the unforgiving plastic chairs of the Community Service Office, nervously glancing up frequently to make sure I didn't miss my number and the chance to ask why we weren't notified about our appointment to review our application for food stamps. I caught myself frowning over Giroux's (1991) careful deconstruction of modernist and postmodernist thought and the possibilities afforded postmodernism by feminism only to realize that I should be smiling at the plump toddler wobbling her way

${ }^{22}$ This, for better or worse, is the main reason I don't have an MSW. 
towards me as I sat in the waiting room that houses both WIC clients and patients at our community health center. I typed and revised these words at my desk, my eyes filling with tears of sadness and joy, while in the next room, a social worker extraordinaire played the guitar for my father in law as he faced his last weeks of life. My relationship to social work is complicated, and although I am often reminded that I will be earning a doctoral degree in social work, it's clear that I will always have a place on the margins of social work.

Miller (2013) wrote of the importance given to the role of reflexivity in social work practice, or at least the role of understanding our "location" in relationship to clients. Social workers cannot avoid judging their clients, but should be conscious of the judgments they are making. However, Miller pointed out that this reflexivity is not always present in social work research: the epistemological question of how we are related to our work is not always addressed. Articulating this connection involves reflection on identity that isn't superficial, but includes one's own history. Here it's useful to include his quotation of Kincheloe and Steinberg (2008), who describe identity as "recognized as the result of arousing submerged memories. A confrontation with dangerous (uncomfortable) memory(ies) that change our perceptions of the forces that shape us in turn moves us to refine our worldviews, our ways of seeing (p. 146). Not only does this reflection involve examining aspects of our identity (for instance, gender or class), but it also involves asking how those identities are lived in our bodies. Knowing who you are, Miller argued, clarifies your ability to know the experiences of another. It is in this vein that I considered my experiences on the margins of both 
education and social work, and my own experiences as a first-generation student led me to ask: What are the conditions of relational life for other first-generation students in a school of social work? (How) does the education change relationships with family members, and what are the implications for professional socialization?

\section{Ethical Considerations}

A feminist stance towards research is often assumed to involve a fundamental concern with ethics. Preissle and Han (2012) noted the assertion by some that feminists promote an ethic of care over the standard ethics of principle assumed in social science which guide research practices and institutional review, reviewing Gilligan's work on the moral decision making and women's valuing of relationships (as cited in Preissle \& Han, 2012) and Nel Noddings's (2002) writings on caring and social policy regarding homelessness. But assumptions about the interrelatedness of caring for others and feminist work are not enough to ensure ethical treatment of others in research relationships. This study was designed to meet the guidelines prescribed by Portland State University's Institutional Review Board (IRB), including informed consent, protection of participants' privacy, and the right for participants to withdraw their consent for participation at any time. However, following Preissle and Han (2002), I also attended to the broader issues of ethics of research purpose, ethics of roles in research, and ethics of representation.

The ethics of research purpose are fraught with tensions about the role of values in pursuing research as well as the more mundane (and often invisible) question of the important role that research plays in promotion and tenure. While we speak about the 
potential benefits of research to the participants, we rarely mention the crucial role that research plays in the livelihoods of those conducting it. Both our political positioning and career interests may introduce tensions into the research process. I have written throughout this section of my discomfort with an apolitical stance: I hold that access to education is important, and that the relational experiences of first-generation students in social work deserve attention because I believe that schools of social work have an imperative to examine their pedagogy, curriculum, and practices in supporting underrepresented students, not only for their scholarship, but for the recruitment and retention of a diverse group of social workers and professional helpers. Like Fine (1992) then, I argued that rooting my work in a politics of access maximizes ethics. Like Crenshaw (1995) and Collins (2000), I argued for attention to the attending to differences in racial, class, and other backgrounds in shaping the experiences of students, rather than a feminist ethics that assumes that all women share the same goals. I am less at ease with the question of "for whose benefit" in pursuing this work. I have imagined these focus groups as beneficial to other first-generation students in social work because of the healing and transformation I've found in talking with other first-generation students and discovering our shared struggles to narrate what exactly it is that we're doing to our families. But I realize that any benefits participants stand to gain from this research pale in comparison to the benefits I will gain from completing a doctoral degree.

Preissle and Han (2012) wrote of the simultaneous emergence both feminist criticisms of conventional research methods and the installation of IRBs in the United States, in response to the same problem: gross misconduct on the part of scientists who 
exploited people as "subjects." IRB protocol is focused most clearly on guaranteeing ethics of conduct in research and clarifying roles. Participants were informed about the potential benefits and risks accompanying their participation in the study, which included the risks of a breach of confidentiality that are part of focus groups more so than in other forms of data collection, such as interviews. Participants were given the opportunity to select pseudonyms, which were used in the transcripts and in representations of the work in presentations or publications. Participants were also invited to participate in group member checking and I've remained in contact with all participants and invited them to approach me with questions or to make more time with individual member-checking if desired.

But Preissle and Han (2012) wrote of other, more subtle aspects of these roles: how is the researcher related to the research participant? How much control does the participant have over the ethics of the study? How does power differ between the researcher and research participants? And how does the research relationship end, and by whom? Although I have written throughout of my attention to relationships and awareness of reflexivity, it would be naïve to assume that these ethical struggles will not color the research relationships. To this end, I included member checking and peer debriefing as part of the methods and selected a method of data collection designed to diminish hierarchical relationships and shift authority to participants. I also included an invitation for participants to choose to remain in contact following the research (via email) as recognition of the inescapable imbalances of power and the value of attending to relationships. I was encouraged by the number of participants who attended member- 
checking sessions and the handful of participants who I've had more extended conversations with following our focus group discussions. While I cannot erase the power imbalance inherent in our relationship, I have attempted to remain available to participants and informed that they will be welcome to ask questions and attend the final dissertation defense. Many of them, while very busy, remain interested in seeing their experiences embedded in a larger story among other first-generation students.

Finally, the ethics of representation involved the ways that we as researchers construct and share the lives of others (Preissle \& Han, 2012). These authors asked:

Will research participants be distressed when they learn how they are described, characterized, and interpreted? Will they agree with how they are represented? Will individuals be placed at risk from others in their situation or from the general public by how they are presented? (p.596).

As a white, heterosexual woman who enjoys some of the privileges of middle-class status, it was especially important that I attended to the ways that my experiences of the world shape my interpretations of another's. In this respect member checks and peer debriefings were also important for ensuring that I was attending to the needs of participants to exercise as much control as possible over the pictures of their lives and relationships that are constructed from this work. 


\section{CHAPTER 4: FINDINGS}

In this study I've broadened the focus from questions of integration in college to explore the ways first-generation students work to maintain connections with others in multiple contexts. I was interested in their connections with family and friends and in their communities, as well as in school. First-generation students have often been framed in terms of their academic risks and literature exploring their relationships has revealed struggles to "integrate" in higher education, therefore highlighting their status as "outsiders" who need to adjust to school culture. The fact that there is a distance or difference between the cultures of school and home is implicit in conversations about first-generation students, but rarely explored.

The focus on first-generation students' academic and social integration into college reflects the dominance of Tinto's Theory of Student Departure (1975, 1993). While integration into college influences student retention, focusing only on students' experiences in school ignores relational experiences outside of the school setting that also merit attention. The focus on integration underscores the fact that some students must do extra work to integrate into college, an assumption which reflects Bourdieu's (1986) ideas about embodied forms of cultural capital, especially the attitudes, beliefs, and lifestyles valued by those in higher education. I've presented these findings as a means of furthering the conversations about first-generation students. First, it's important to expand our focus beyond integration in school and see the work students are doing to remain integrated in their home cultures. And second, it's important to build on Bourdieu's (1986) ideas about cultural capital both by providing concrete examples of the 
forms of valued cultural capital students are working to learn (the "hidden curriculum") and by examining how institutions of higher education are implicated in maintaining separation between cultures of home and school.

Gergen's (2009) ideas about relational being provided additional framing for this work and I've used his terms throughout the findings, attempting to draw parallels between the experiences of integration and his notion of "remaining intelligible within relationships" (p. 140). We think, speak, and behave in ways that keep us relevant to the people in our day-to-day lives. For first-generation students much of the focus has been on the work students do to integrate, or become intelligible, in college. This took work for students, as they did perceive a distance between the cultures of home and school and had to embrace new ways of thinking, speaking, and behaving. But students were simultaneously working to remain intelligible in the worlds of home as well.

In these conversations with 19 undergraduate and graduate students in a School of Social Work (SSW), students tended to endorse the notion of a distance between home and school cultures. But rather than center their attention on integration, students strove to also remain relevant within their home cultures. I'd often conceptualized my own experiences as a first-generation student as that of crossing bridges that connected the different parts of my life. But listening to the voices and stories of these students revealed how much students were the bridges between the cultures of school and home. At school these students had to learn the dominant cultural ways of being, which were new to most students I spoke with. In doing so, many of these students saw themselves as creating new paths for others in their family or community to follow, and rightfully so. But 
learning the dominant cultural ways of being which structured their learning and interactions in college often resulted in new tensions, shifts, and ruptures in their relationships in home cultures: students risked becoming "unintelligible" in their relationships with family, friends, and members of their communities. Losing these connections to any great degree was untenable for the students I spoke with, and therefore they worked to stay relevant to home as well. Not only were students traveling back and forth on the metaphorical bridges I'd constructed to understand my own experiences: they were bridges, stretching to find new, solid foundations in the school while also working to maintain their foundations in the home cultures they'd come from. In each space the students occupied a one-down position of power: in school they learned someone else's culture and at home they worked to stay intelligible; for many students, the transformations prompted by schooling or identification with a professional role marked them as different and distant from those they'd previously been close to.

In the first section of the findings I've addressed the primary research question, which focused on the relational worlds of first-generation students in a school of social work. In keeping with the bridge metaphor, I began this section with experiences of support from home cultures, which carried students into the middle of that distance between the worlds of school and home. Students were largely on their own in pursuing school, particularly in terms of instrumental forms of support and others' understanding their daily lived experiences. There was a great potential for distance from home cultures and some students did feel distance(d), but students spoke much more about the work they did to remain intelligible in their home cultures. While most of my focus was on the 
relational work students were doing to stay relevant within their home cultures, I've also explored their experiences of becoming intelligible or working to integrate in school culture as well.

The second section of the findings focused on the implications of these relationships for the process of professional socialization. Here too students spoke about messages from home that supported the work they were doing or learning to do, but oftentimes elitist biases were wrapped up in their descriptions of social mobility (in contrast to work their family members had done or that they'd done in the past). Students also spoke about the tensions of creating new routes: paths into work and schooling that were unfamiliar or inconsistent with the experiences and expectations of those closest to them. Gender, culture, class, and family history were powerful factors in the blueprints for the lives students were often expected to lead, and students often had to diverge significantly from others' ideas in their career choice. Their stories highlight the ways sexism, classism, and racism structure educational opportunities - and potential barriers. Family voices often remained powerful, though, in shaping students' notions about their professional roles, particularly for social work students. Finally, I explored stories of exclusion in field that were especially prominent for some first-generation students of color.

In closing I’ve argued for framing first-generation status as inherently relational: while students do often embrace the values of individualism that permeate traditional notions of student hood, students made meaning of the first-generation experience through a lens that was largely family-oriented. Here I've also written about our 
dominant cultural narrative of college as a way of moving up and the potential for relational injury, when this rhetoric and our practices in the academy are unexamined.

Throughout the findings I've included excerpts of the transcripts from these conversations to illustrate points and provide context for my interpretations. I've attempted to personalize the speakers by identifying which degree they were pursuing and using the demographic details they provided. I've also included formatting from the transcripts that aids understanding of the conversations. When participants emphasized certain words, I italicized them in the text. As much as possible I attempted to capture tone and emotion in speaking, providing notes in brackets after the word or phrase. In some sections I've included my voice as the interviewer; in exchanges where I speak, my contributions are italicized. As has been true throughout this study, my voice is embedded in the description of these findings. And while I attempted to minimize my voice in conversations with students, I know that it is neither possible nor desirable to abstract myself entirely from this work. Instead, here I will attempt to be transparent with my voice as I frame the relational experiences of students in a school of social work. 


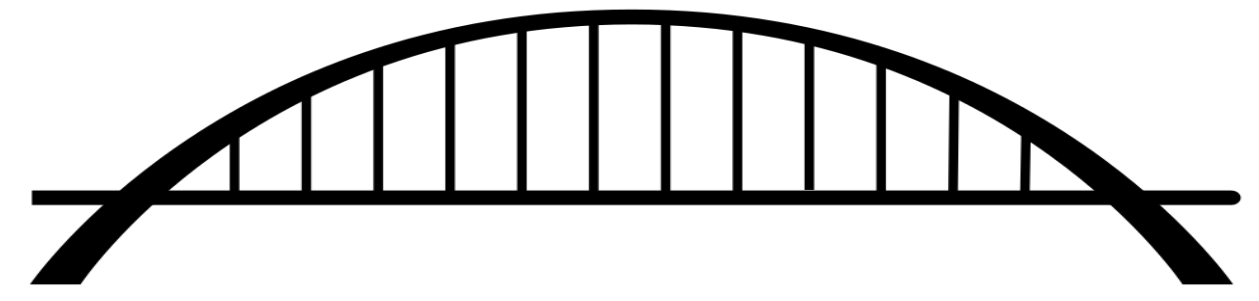

\section{Home \\ (family, \\ friends, \\ community)}

Working to stay integrated in home cultures (pp. 185-203)

- code-switching and self-silencing (p. 185)

- navigating between worlds (p. 195)

- self in relationship:

careful self-presentation (p. 198)

\author{
Experiences of \\ support \\ (pp. 140-147) \\ - mixed messages \\ (p. 141) \\ Pursuing school on \\ your own (pp. 148- \\ 162) \\ - financially on your \\ own (p. 148) \\ - figuring it out (p. \\ 154)
}

Potential for distance (pp. 162-185)

- "conversations we can't have" (p. 166)

- responses to

communication

breakdowns (p. 179)
School

(students,

teachers,

classroom)

Working to integrate:

Relational experiences in school (pp. 208-228)

- mystification of the university (p. 209)

- relational experiences in school (p. 213)

- in the classroom (p. 223)

Figure 2. Visual Outline of Primary Research Findings 
RELATIONAL WORLDS OF FIRST-GEN STUDENTS

How do First-Generation Students in a School of Social Work Describe their

\section{Relational Worlds?}

\section{Experiences of Support}

Students were quick to acknowledge how family supported their education. Usually this support took the form of encouraging words or praise for achieving something other family members had not been able to. Here Lizette, a working class Latina and CFS student, described the support she received from some family members:

...I get the comments "Keep going," "Do you need help?" "Do you need money?" "Do you need any thing?" I don't have my family around. I have them all spread out. I have parents in California, my one only brother that's left who's in Missouri, and it's like, "need anything?" and I'm like “No, it's okay" “Are you sure? Do I need to fly out?”...And, I can say, just last week my mom left, she was here for three months with me helping me watch my boys, it was a relief.

Dave, a white working class MSW student, spoke at length about the various ways his family supported his education "financially, emotionally, spiritually, psychologically" and noted, "I wouldn't be here without my family." Clara, a white lower-middle class MSW student, was one of the few students who spoke about family providing instrumental forms of support in the process to get into school: 
RELATIONAL WORLDS OF FIRST-GEN STUDENTS

Neither of my parents even did an Associate's, and they were both like, my dad was willing to help, but they were like, "We're just going to figure this out together. Like I don't really know any of this stuff.” So we would go on the computer together and do the financial aid and that was really helpful

Juli, a white lower middle-class student completing her BSW said, "in my family...it's a big deal. Like, 'Look at you, you're going to school. You're gonna get your degree.'” Bob, a working class Latino in CFS, described his father's interest in the classes he was taking, “...over the phone he'll ask, 'Oh, what classes are you in? What you learning about?' He always gets really attached to it...he's always saying, you know, 'I wish I was you.'” In general, students' families supported educational attainment as a means of social mobility and ensuring economic security. Our cultural narrative about higher education links a college degree with middle class status and a stable income. Family members were sometimes unclear about aspects of students' degrees or the work they might do, but most family members encouraged students to continue. Dave's father told him “I don't want you working in a sweatshop like I did...I want you to gain more. I want you to be more than what I was," and Dave concluded, “...there's a support coming from that."

\section{Mixed messages of support.}

The support students received from family was often mingled with concern, questions, and challenges from those same family who supported them. Students noted their families' cautions about debt, queries about careers that felt unfamiliar (or threatening, in some cases), and concern for the amount of time students dedicated to 
schoolwork. Families' mixed support may have also been a response to changes in students' ideas or values, particularly in relation to conversations about privilege, oppression, and aspects of identity. Here Amber, a white working class CFS student, shared her father's advice, which suggested that she should pursue work she loves but also raised questions about the wisdom of her choices:

my father's like "How are you going to pay off those loans with your fluffy degree?"...I see him, and then he turns around and he says, "Don't do the main dang job just to bring home the paycheck, you know, do something that makes you happy." So he kind of reverses it. So it's...very contradicting.

It seemed that multiple concerns were wrapped up in Amber's father's words. Amber will be the first in her family to finish a Bachelor's degree, but her presumed social mobility may be hindered in her father's eyes by the gendered nature of the work she will be doing and the de-valuing of labor in fields dominated by women (Cohen \& Huffman, 2003; England, Allison, \& Wu, 2007). A second thread in her father's mixed support is clearly tied to fear about debt. While parents see college as a path toward economic security, the debt loads students often assume gave them pause. Many students raised the issue of debt in their conversations as something that was both a source of concern for them and family and that students accepted as the price of getting a degree. Here is an exchange between two white working class MSW students, Michelle and Nancy, that highlighted student feelings about debt, a subject which emerged unexpectedly from discussions about their experiences of feeling different from other students in terms of social class: 
RELATIONAL WORLDS OF FIRST-GEN STUDENTS

Michelle: ...I know like my undergrad there was a ton of people that were kind of just like flaking school off and having fun, and I was like "You guys are totally taking it for granted, I'm paying to be here-" [laughs]

Nancy: -and I'll be paying forever [laughs, all laugh].

Michelle: And that's terrifying for somebody who's like, already not affluent...um, to accumulate more debt before you even...

Nancy: Have your real job?

Michelle: Yeah, yeah.

While this excerpt highlighted students' feelings about debt, it's not hard to imagine these feelings mirrored in family's concerns as well. Michelle was wise to point out the role social class and economic resources may play in someone's reluctance to take on the large amounts of debt which have become commonplace for students.

At other times family members' messages about higher education suggested ambivalence about the value of a college degree in general. For these families, experience working in occupations that didn't require a degree often shaped messages to students about the necessity of a degree. Jaclyn, a Native American and white working poor MSW student, noted her parents' skepticism of higher education, based on their work in jobs that require manual labor, "My dad's always told me, 'Who would you rather have dig your ditch? Someone who's read about it, or someone who's done if for 20 years?'...It's not that they don't agree with college, they just don't think that people need to go to college." Sometimes family messages became more supportive over time. Her mother initially questioned Tara, a white working class CFS student, when she went to college. 
Her mother, who had found "her own success" in restaurant work, initially told her “Well, you just work hard. You don't need a degree.” But Tara explained that her mother had "come around to realizing how important it [the degree] is to me, and that's what I want, to be able to have a job that's meaningful to me."

Nancy, a white working class MSW student, articulated the ambivalence in her family about her bachelor's degree and the graduate degree she was currently pursuing:

I think within my family it's really complex, the way that my role as a student plays out. 'Cause like with my mom and my stepdad it's like, a really, I think it's an important piece of my identity to them, because they want that for me, and they know that's what I want. But outside of those relationships, my role as a student is--really feels like a negative thing.

Later in the conversation, Nancy expanded on the role that gender probably played in the mixed support she received from family members:

...I guess-I was gonna say, I also think gender has been a big piece too. Like, I've gotten questions from family members like, "Why do you want that? Aren't you just gonna get married?" Like, for real, I've heard that...'Cause all of my cousins - female cousins are stay at home moms, and I have like, twenty [laughs, others laugh]. And they're really perplexed by this idea that I want to-do something different... 
Similarly, Lizette, a working class Latina who spoke about her family's support above, noted how the intersection of gender and culture in her family shaped the reactions of certain family members to her decision to complete a Bachelor's degree:

Sad to say that only one person that I get the negative from, sometimes, is from my mother-in-law, it's like why - you're a mother - it's because of the Hispanic culture, once you become a mom, you have to drop everything [emphasis on each of these last five words].

Rather than accept this, Lizette contested the idea, "But on the other hand, no I don't. I need to grow... I need to keep going." But she also noted how difficult it could be to continue in her education when facing opposition or criticism:

Lizette: ...my aunts and uncles, I don't really listen to them because all I get from them, like "well, what are you doing that for, what's the point?" So it's like, I don't need to hear the negative, so it's like, toss, close the door, like "How are you?" "Fine." "How are the kids?" "Good.” I'm like, "Anything else?” and they're like, "No.".... ...those family voices are really powerful....

Lizette: They're very powerful. They're very powerful. They think...it doesn't hurt and it does, so it's like, very powerful.

Maria, a working class Latina completing her BSW, noted her family's support and its limits. Maria spoke lovingly of her family's role in her education; her degree was the result of sacrifice across generations of women in her family. But she was also persistent 
in challenging family with new ideas and learning, and sensitive to the power of family responses to those ideas. In this quote Maria explained her hesitancy to describe family as supportive. Their mixed messages of support were primarily responses to her challenges to cultural and gender norms:

So it's like, we'll support you in getting your education as long as you don't challenge what's going on at home. So I say, when I caught myself and I said, “Oh, well they're so supportive!"” and it's like, "Well, kind of." [laughs] 'Cause they're supportive in getting an education but not in challenging what they want, in challenging the norms in my house or in my family...and, so yeah. So general sort, what a woman's supposed to do, what they're not supposed to do. Howother ideas, to think about maybe, disciplining your children, other ideas and you know, perhaps, you know, somebody doesn't want to live at home. And they want to go and live on campus. Or maybe they want to live with their boyfriend before they actually marry the person! [laughs] Um, yeah, so we support you, but to an extent.

Another source of family resistance that may have contributed to mixed support arose from the sheer amount of time students dedicated to schooling. The time schooling demanded sometimes surprised family members. Here Brandi, a working class JamaicanAmerica CFS student, described her mother's mixture of pride and frustration at her schooling:

Um, as far as family goes, too, my family is supportive and it's really funny because like I said, my mom, like in Jamaica, it's like university, you just have to 
RELATIONAL WORLDS OF FIRST-GEN STUDENTS

go to school, like that's your way out. But she didn't know what that meant because she'd never been herself, and so she is supportive, but when I tell her, like I'm still—after like my first two years of school and she's like, "Have you graduated yet?" and I'm like, "No mom, it takes four years to get a degree." And she's like, "Why so long?" [laughs] She's supportive, but she's very concerned because family is so important, and it's—she sees that it's been a struggle for me to go to school, I have not been able to spend as much time with family, so she can be a little resentful of that.

Brandi's mother's response highlighted the mixture of pride and frustration parents might feel. Here Brandi was stuck in a double bind: she was fulfilling a role that was valued in her mother's home culture, but fulfilling that role lead her mother to be "a little resentful" of the time investment it required.

I'll return to family concerns about the relevance and intelligibility of careers in the second portion of the findings, as family ideas about students' careers have implications for the process of professional socialization. The cost of schooling wasn't only financial, though: schooling also exerted a relational cost in terms of time spent away from family. Gender and culture were especially strong factors in family messages that questioned students' pursuit of degrees and the time spent away from family. Women, but not men, said that family raised questions about schooling interfering with responsibilities to family. These concerns encompassed responsibilities to actual existing family members and to anticipated members of families, such as future partners or children. 


\section{Pursuing School on Their Own}

\section{Financially on your own.}

While students felt that their families showed overall support for them completing their degrees, most of the students I spoke with were largely pursuing degrees on their own, at least when it came to financial and other instrumental forms of support for their educations. Sometimes this led students to feel distress and great pressure to succeed. Here Bob, a working class Latino CFS student described his drive to do well and stay in school:

... it's really stressful for me... I don't have that - like a connection, close connections to my family. They're really close, but like...I feel like they're kind of "Just go for it!" [laughter] They're not really like, watching me, they're like, "You've got it." But at the same time, if I don't get good grades, if I don't do-if I drop out, then there's, there's nothing there to get me back up [cups hands together to make a symbol that looks like a net]. If I'm out, then I'm out. And it's like, terrifying to me cause I don't want to go-like this is like a blessing to me because I feel like it's the only thing keeping me from working like 40 hours a week at like a pizza place, and that's where I've been, so I feel scared to like, be here at the same time- 'cause it's like, stressful and like all the pressure, gotta get through this, cause this is like my ticket to being comfortable.

Later in the conversation, Bob shared that succeeding in school was his way out of a "lower class role" where he was "struggling to eat." Because of this, Bob approached 
schooling seriously, seeking perfection in his grades. Here Bob's words also reflected the mixture of family support that included encouraging words ("You've got this!") but stopped short of providing economic support. As mentioned above, gender may have shaped family responses here: Bob's family was close, but not concerned about Bob's schoolwork usurping responsibilities to family. Gender may allow Bob to more comfortably assume the traditional role of a college student as an autonomous individual (Renn \& Reason, 2013). But this didn’t alleviate Bob's extreme levels of stress as he was pursuing a degree on his own. Bob's tension here was visceral, conjuring up primary needs such as eating. While their feelings about money were not as intense as Bob's, the other three students in this discussion also spoke about being financially on their own:

Amy: Kind of like what you said, like if I were to drop out, like my parents would be disappointed, but they wouldn't—like they haven't paid for my school or anything. They wouldn't be like, “Oh, what do you need? Like 5,000 dollars to finish school-“" [laughter]...

Well, and that can fill in the gap: that can be-things like books, getting, or being affordable, when classes start, you know. There can be those-that, that symbol you made with your hands [referring to Bob's hand gesture, cupping both hands to make a "net"] is so meaningful, like there's not that net to be like, "Oh no, we'll help you." Like we'll be, we'll be in this...

Lauren: It's kind of the same for me, too. Like I've-my, growing up, my family we don't have a lot of money, so it was like, “whatever you do, you're kind of on your own in life." I've been really independent since I was like 16, so I knew 
coming here I would have to live off financial aid and my own income. So that's been...pretty tough. But also a motivational...um, thing. I even had to ask my dad-I asked him for like 200 bucks, just to help me with school and he was like, "Yeeeaaahhhh..." [drawn out slowly, suggesting unwillingness], like reluctantly, cause I know it's really tough and it was like, 'I'll pay you back, I promise!'[nervous laughter] —I feel so bad asking for money, but you know it's just-finances are tough.

Brandi: Same. Echoing a lot, the sentiments that people are sharing, that they shared, being culturally, that I shared, I shared with you, in the Jamaican culture, education's really important. But I'm an older student, so traditionally people, like, they would go to university after high school, like in actual Jamaica, and at that time, family's very supportive. They will try to support as much financially as possible... And so now that I'm older I'm doing it on my own, they just can't...assist me.

Like Bob, Amy, Lauren, and Brandi were also financially on their own. All of these students accepted that they were on their own in paying for college; Brandi cited cultural norms that dictated family economic support, which she fell outside of as an older, nontraditional student. Lauren may have even expected it, having been "independent" since she was 16 . Her story about asking for a loan illustrated the implicit agreement she had with her father: college was her financial responsibility. Lauren highlighted her possible transgression of this agreement by asking for a relatively small loan. It seemed that parents also felt that it was students' responsibility to pay for schooling. 
While many students didn't question the fact that they were financially on their own when it came to paying for college, they occasionally imagined how it could be different. Some students compared their experiences working through school and paying for school to those of their peers whose families paid for them to go to college. Here two white working class CFS students, Amber and Tara, spoke about yearning for the same levels of family support that they saw peers receive:

Tara:... I graduated high school in 2007 and most of my friends actually had, like, pretty different experiences than me. Like, their parents were, like sending them to college and like, I was the only one of my friends who was working at 16 . So, I felt like...uh, kind of like a separation in that way where I was like...kind of wanting to fit in with that, and like, wishing my parents would...support...that, but...

Amber:...Uh, yeah, I...majority of the people I met at the [state flagship university], the first try at college [laughs], everyone I met, you know, they didn't have to work. I worked a full time job and went to school and eventually just couldn't do both. Quit going to school and just worked full time because the economy was great; I had a great afterschool program that I was working at. But, the majority of my friends that I met in the [state flagship university], their parents were paying for their school, like my cousins, both of them, their parents told them, you know, "No, don't work, just focus on school” and I got so envious, like "Oh, that must be nice" [Lizette and Amber both laugh]. "Just going to school, and just focusing on that." Like, getting into your first choice school too. 
And, you know, money not being an issue. You know what I mean? [Lizette laughs]. Like, getting in, that's a whole different story. But, like the economics...like, "Oh, you know, you want to go to the East Coast? Go for it!" And it's like, "Oh, that must be nice" [everyone laughs].

While these students laughed at the unlikelihood of being told they could study wherever they wanted, they also highlighted potential benefits and drawbacks of being on their own in pursuing an education. Tara spoke of her friends' parents' substantial support in getting into and through college and said, "It was kind of hard to deal with at the time. And I feel like - now it almost like, gives me a sense of pride, though...I had to try really hard to get to this point, and I've been able to do it on my own, so...I think that's a point of strength." Amber agreed that it could lead to feeling proud and accomplished, but her next words revealed a potential danger for students to locate failure in themselves rather than recognize how difficult it is to complete a Bachelor's degree on your own: "I felt like, 'Well, everyone else is making this work, why can't I?'” This point is important to notice. Students realized they were doing something with far less support than many of their peers and were well aware of the difficulty. But when students spoke about potential failures (such as the possibility of dropping out or taking "too long" in school), their explanations omitted these contextual factors; students were ready to accept full responsibility for failure. And while most parents and students seemed to agree that students were on their own financially, several students said their parents had expected them to go to college. Amy, a white lower-middle class CFS student, confessed that both 
she and her parents had always expected her to go to school, and she felt she had let them down when she took longer to complete a Bachelor's degree than expected:

I feel like my parents always expected me to go to school, and I always expected myself to go to school, um. So when I took the-12-year-break [laughs] there was kind of, sort of disappointing to them, I think? Cause they always expected me to go to school but never really like, did any sort of — helped me with any-cause they didn't know how--you know, they didn't know how to apply for FAFSA, or apply to school. They just didn't have any experience with that, or, or maybe that or, I just, I think that their lack of experience sort of made me, slowed my process down a little bit, even though it was, I always felt the expectation...you know, they didn't know how to apply for FAFSA, or apply to school. They just didn't have any experience with that...I think that their lack of experience sort of made me, slowed my process down a little bit, even though it was-I always felt the expectation...I don't know if that sounds weird [laughs].

Amy realized that her parents were unable to help her but also felt disappointed by her inability to meet their mutual (but unstated) expectations. It's also important to note that it seems like both Amy and her parents felt that college was something that would just happen. This doesn't seem like an odd thing for families and students to expect, given that this is largely how education operates for students during the first 13 years of compulsory education. This process seemed shrouded in mystery for family and students, and this left families, and particularly students, to struggle through this on their own. Jayne, a white lower-middle class non-traditional student completing a BSW, shared a 
similar story about her first attempt at a college degree. "It was just an expected thing in my family," Jayne said about college But Jayne also noted that when her first attempt at college, "a private college that was not accredited" where "there were no degrees there for women...not in '78" didn't pan out, she accepted her fate, thinking, "Well, you threw away your chance.” In this same discussion, Veronica, a Mexican American student completing her BSW, invoked expectations the elder members of her family had for her and her cousins to attend college, which were folded into the narrative of immigrating to the United States to provide a better life for younger generations:

Between me and my cousins, we're all like, first-generation and grandkids, and like, U.S. citizen, born here, um...for us, at least, it's like, I don't know, like based on family reunions and stuff like that, I think that the things we're saying and the jokes that we're making, we're--I guess to older adults it would seem like they're like, 'Oh, I want the children to go to college. They're gonna succeed in college," whatever succeeding meant to them.

Veronica's words, "Whatever succeeding meant to them," were notable. Like Amy and Jayne, it seemed like families often had vague expectations that students would go to college and earn a degree. But when parents don't have experiences with the process of in applying to, being admitted, and enrolling in college, those expectations may not be enough to translate into college access.

\section{Figuring it out on your own.}


Pursuing schooling on their own manifested in other ways, including getting into college, figuring out how to pay for it (which is separate from the act of paying), and arriving on campus and finding one's way. Michelle, a white working class MSW student, felt that feelings of being on her own were most acute during her undergraduate years, and explained the feelings of being "behind" other students:

I didn't really have anybody to talk to about how to go about just navigating the system, and it was really all up to me, and my parents were just, they were pretty clear about that. They were like “we don't know what college looks like, we don't know how to apply for financial aid, or sign up for classes or anything like that, like that's all you if that's what you want to do, so...do it.

When students spoke of their family's encouragement and support, they often balanced this with their families' lack of knowledge about college, though. Maria, the working class Latina BSW student who spoke about her family's measured support, also noted her family's lack of understanding about what a Bachelor's degree meant. And Dave, the white working class MSW student who noted all the ways his family supported him, said “...they support the notion of education, but conceptually they don't understand what that entails." Family knowledge about higher education, or the lack thereof, speaks to embodied forms of cultural capital (Bourdieu, 1986). Familiarity with the progression of different levels of college education, knowing which questions to ask, and anticipating transformations students may experience are all pieces of cultural capital which may support student integration. Several students expressed sadness at not being asked about their studies or what they were learning. But the bigger relational impact for students 
arose from their family's lack of awareness of the time demands of college. As mentioned earlier, women were more likely to be questioned about taking time way from family roles. At other times, family or friends sometimes assumed students were snubbing them when they were actually straining to meet the demands of college, and often employment as well. In this discussion between CFS students, Lizette speaks to the weight of trying to fulfill multiple roles with competing demands, as a mother, spouse, worker, and student:

it's...consuming. I feel like, I also work, it's like, I feel like my time is taken up, and that I... at times I guess I feel guilty. And I don't want to feel guilty, but it makes me feel guilty because my kids are young, I should be there more, but I'm focusing on this, so I'm trying to deal with time, I'm learning in the process how to manage school, family, and work. It's just, I'm still trying to figure that out. It's just very consuming. I had my mom over, and she was like, "do you realize you're not asking your children how their day was, you do realize you're missing these little steps" and I go "no, I did not realize that." I mean, her [sic] said that to me, and I'm like "I am, I should be asking my four year old, 'how was pre-K today? What did you do?' Playing with my youngest, who's one and a half. Talking words. It's like, okay, I'm missing these little steps. So I'm learning about time.

Here Lizette's language is telling: it's "consuming” to fulfill so many demanding roles. And here she faced the added task of explaining to her mother, perhaps even justifying the time she spends on school, which was taking her attention away from her children. At 
hearing Lizette's story, Tara said "I can't imagine having kids at this point, when school is so consuming," and noted that she didn't have friends at the moment, aside from a close friend and a boyfriend: "it feels impossible to have any other social relationships." Amber followed this up, pointing out an important relational struggle for students whose friends aren't in college:

It's so hard because a lot of my really close friends that I've grown up with never went to college, they just went right to the workforce, so I really struggle with finding that common bond. And I think that's why I hang out with my partner so much is because we both met at school. So we have that common identity and that common understanding. Now he's working, he's no longer going to school, he graduated already, but like, just that piece is there, so like if we don't spend a lot of time together, he understands why not, whereas my other friends are like, "I never see you anymore, you're always with your partner" “No...I'm doing schoolwork. I don't really see him and I live with him!" [Amber and others laugh]

Notice here the potential for relational discord: her friends assume she is blowing them off to be with her partner because they don't see her and expect her to have free time. Lizette's voice was heavy as she described the "consuming" nature of all the roles she had to fulfill. Her mother's help was a form of support, but also came with questions about the time Lizette dedicated to schooling, which took her away from parenting. It posed a potential relational strain between Lizette and her mother: Lizette clearly wanted to do the things her mother thought she should be doing with her children, but felt pulled between the demands of each role. The time demands of school often extended well 
beyond the nine to five workday, even for students who weren't working in addition to going to school. Davis responded to Amber's description of her struggles to remain relevant to friends by highlighting the influence of family and friends and their expectations for life after high school, which are often intergenerational:

Davis: Yeah, it seems like that has something to do with your generations of people going to school, you're kind of conditioned to juggle these things too. And so that's confusing when it happens, because you're not used to that kind of program...you have different, you know, priorities. [voice gets very soft] You know, having children, having a girlfriend....many people right after high school, they just go right into it, this is gonna be my life.

Gotcha. So Davis, when you say "generations," you mean, it kind of depends on your age, it kind of depends on where you're at when you go back, and whatDavis: Or just your generations of people who have gone to college who can maybe reflect on their experience.

Oh.

Davis: So that you can, you can relate to them. And it feels normal. Gotcha, yeah...

Davis: And that's just, what you do.

Yeah. Yeah...

Davis: So, yeah, that's a kind of hard thing. Like, hard realization. To not just be working. Like, working, going to school, and struggling with time management... 
This phrase seemed powerful, "to not just be working." In addition, Davis located the struggles in his experiences as an individual, he was "struggling with time management." Collier and Morgan (2008) wrote about first-generation students' struggles to learn the student role and highlighted "time management" as an especially difficult task for firstgeneration students. It may be that this struggle is amplified by the expectations of family and friends who haven't experienced the student role.

In another conversation between CFS students, Brandi explained that her mom had mixed feelings about her schooling because of the demands schooling placed on Brandi's time, taking her away from family. "In my culture, when people - if they don't go to college, they're working hard, but they're working in the family, hard. And you all know, when you're in school you have a very demanding schedule. It's hard to give one hundred percent to your family. So that's been hard for them." Notice that in the conversation Brandi can rely on her fellow students to understand the amount of time it takes to be a student ("you all know, when you're in school you have a very demanding schedule"), something that leads to misunderstanding and "resentment" on her mother's part. The fleeting connections that arose in conversations offered support for many students, who seemed glad to be with others who understood the demands placed on their time as students and the strains to remain connected to family. Amy offered her experiences with the same issue, albeit more light-hearted, "...my mom will send a text to me, and say "Oh, can you call me right now?” And I'm like, “Oh, I'm in the middle of writing a paper." And I know she's probably like, "How can she always be in the-“ [everyone laughs] “-middle of writing a paper?” But it's like, I always am.” This 
frustration was also shared by Lauren, who said “...it is frustrating because they don't understand. It's like, I literally am going, like seven days a week, 24/7, I don't have a break. And they're like, 'Well, you must have time for something.' Literally, I don't." This breakdown in expectations about free time has important relational implications. Amy explained, "I feel like, you know, I get the vibe from them that they're like, 'Really? Like, you really don't have the time to talk to us because of school?' It's not like, that intense, but it's just 'cause they, you know, they haven't, they haven't gone through it, so...they don't understand that." In another conversation with MSW students, Michelle and Nancy spoke about the strains on relationships with family who aren't aware of the time they need to spend on their work and internships:

Michelle: ... I think especially, when your family is not-when they haven't experienced higher ed and neither have your friends in your community, like seeing you just like constantly being busy, and I think that that can put a huge strain on relationships, 'cause like it's hard to understand if you haven't lived through it, and if you don't realize that that's like part of grad school or part of school, um, it can, it can be taken on and felt really personally.

Nancy: I hear that, ‘cause I feel like, I'm never available_-very rarely. And I've had like my parents say, "Well, you're never around." Well, I can't be!

Again, gender seemed prominent in these women's readings of family messages about spending more time with them. Brandi, Lauren, Amy, Michelle, and Nancy were all well aware that they were strained for time. However, their families' questions about how busy they really were posed challenges to their relationships. Brown and Gilligan (1992) 
wrote about the "experience/reality" split that women often endure in service of relationships. Socially constructed notions of femininity pressure women to prize relationship with others, so much so that self-silencing of one's own reality to accept someone else's version becomes common, beginning in late childhood and early adolescence. These women's family members expressed disbelief at the fact that they couldn't be more available for them. It's not clear if this disbelief led these women to doubt their own assessments of how busy they were. But the desire to fulfill those roles and remain in relationship was evident.

In the next section, I'll return to this and pick up ideas around the power of the expectations and experiences of our family and friends in influencing career and educational paths. What does it mean for first-generation students to pursue a different path than family and friends and enter college, which places great demands on their time? How do these students remain relevant in the lives of people whose experiences look so different? Nancy, a working class white student completing her MSW, conjured up an image of divergent paths when talking about the distance that now existed between her and her family members, “we all, kind of, picked our future routes and so we just don't connect about the same things we used to, even if we have the same things in common now." The image of the "future routes" is apt here: students who take an unfamiliar path to a college degree, and particularly a graduate degree, may diverge significantly from the paths their family and friends take.

Aside from the time demands and relational strains placed on first-generation students, these students also felt that they were leading daily lives that felt largely foreign 
to family. Similar to the women speaking above, Jaclyn, a Native American and white working poor MSW student, expressed her frustration at being asked the same questions each time she saw her family, "if they call me or I go visit and they'll ask me what I've been doing, it's almost like they don't understand how busy I am or what my life consists of, and even when I've explained it, it's like they don't listen. Because a month down the road when I come visit again, they'll be asking the same questions." Here I suspected that Jaclyn's parents may have needed to keep asking questions because her life was so different than theirs, and in member checks she confirmed this: the life she was creating was completely unfamiliar to them.

\section{The Potential for Distance}

Even students who spoke highly of family support noted the relational distances inherent in the process of completing a degree. Because students were largely pursuing schooling on their own with daily experiences that were unfamiliar to family and friends, there was the potential for great distance from home cultures. Dave, who spoke often of the support his family gave him, also said “in some way, I feel like I've...distanced more from my family. And I think that is the result of having a critical self-reflection of where I stand in society, in terms of, you know, race and ethnicity and gender and sexual orientation.” Arturo, a working class Latino who was a non-traditional BSW student, spoke about this distancing from family:

So it's like, I don't have the same...relationships with some of my brothers, or-even my brothers, let alone...friends...They still...work, they work. I'm a working-class citizen; they're all working class. So they, our relationship change 
RELATIONAL WORLDS OF FIRST-GEN STUDENTS

is another challenge... and when I go out, my friends they aren't my friends anymore.

Arturo attributed this distance to how learning about privilege and oppression had changed him, saying that "social justice language" had changed his thoughts and words. Arturo's family and friends noticed the changes in his speaking, and Arturo noted that he no longer enjoyed their conversations as much as he used to: talking about family problems or discussing sports with friends or neighbors had lost its appeal.

Jayne, a white lower-middle class BSW student, described a distance between her and a sibling which she attributed to class mobility, "religious," and "philosophical" distancing. Jayne wanted to connect with this brother, whose distancing from her was also mingled with disapproval:

Well...I have one brother, who has succeeded...in moving up, up the socioeconomic ladder of our family, which he's very proud of. [laughs]. But he can also be kind of offensive with it, so. But, hey, whatever! We, he, we had a funeral... an aunt, die. So...he doesn't talk to me very much because he doesn't, can't, um, religiously or philosophically align with me anymore, um...[laughs]. So he comes to the funeral. And we have the funeral, and we're gonna drive to the cemetery, so I'm like, "ride with me!" you know? So he comes to the car, and he's like, "So did you drive up after work?" And I'm like, “No..." I said, "I don't work.” This is how, how far apart we are. "I'm not working."

"Well, what are you? Independently wealthy?" 
I said, "No, I'm in school."

He said, "Oh, let me guess. You're gonna become a social worker." [5 second pause]

I said, "Yeah, right on. How'd you know?"

Here Jayne told a story of class mobility on her brother's part, something that she later revealed he both celebrated and felt uncomfortable about, of distance in religious and philosophical persuasions, and noted both her vulnerability and power in the exchange. Even as Jayne shared that she was saddened by his disapproval, she also drew a sense of power in exposing how far away her brother had drifted, becoming unaware that she was going to school. At the same time, Jayne dismissed her brother's actions ("But, hey, whatever!'), and endorsed the idea that she didn't need his approval or support to succeed as a student.

In a discussion with CFS students, Lauren and Bob spoke about distancing from people outside of their families, in one case people in the "lower class town" where Lauren grew up and Bob's growing distance from his friends who didn't attend college:

Lauren: --I think it depends on the setting. At least in [nearby upper middle class town] it's very meaningful for me to be a student. Whereas I'm from [nearby lower-middle and working class town] where I think they would kind of be like, “Oh, you're at college?" [clicks tongue] "Good for you.” [frowns, feigns disdain]... And I'm, I'm kind of making assumptions about what you mean by "Oh, " [clicks tongue], you know? 
Lauren: Yeah, they just...

\section{Can you say more about that? What do you think that reaction is?}

Lauren: I feel like they're...threatened or something, cause they didn't get that, you know, opportunity that I have to go to school. Or at least they didn't... want to, to get themselves out of the lower class town...

Bob: Um... when I go back, I'm going back to visit my friends in Georgia in June. And I know when I go back, I'm going to be treated really differently. Because none of my friends went to college. And I haven't lived there in like, five years I feel like, or four? But I know that, um, I know I'm gonna be treated, like, really differently, cause I know their attitudes towards college is very negative, I feel like. With my family it's a little different, because with my family I can talk to my parents about it, and I won't be treated differently, but in Georgia I'm going to have to, like, put on a face, I feel like, for it, cause there, college isn't the way they're...feeling.

Here I was struck by the different ways Lauren and Bob were experiencing others' reactions to their class mobility, which was an assumed part of going to college. Lauren was aware she was "threatening" to people in the town that she grew up in. At the same time, she was looked down upon for going to school: people might mock or dismiss her as a student. There are interesting tensions for Lauren, who seems to want to still be accepted but also seems to blame other people for not being able to "get out of" the "lower class town" she came from. Bob maintained a comfortable closeness to his family, who were excited about his learning. But he expected his friends, who hadn't attended 
college, would feel "negative" about it, and was preparing to downplay college and "put on a face" to minimize potential distance.

\section{“Conversations we just can't have"}

While some students described relational distancing, it was much more common for students to talk about experiences of struggling to remain connected to family, friends, and community. A large part of this effort was focused on conversations. When speaking about possible differences between the cultures of home and the cultures of school, conversations were frequently mentioned as a point of difference; indeed there were some conversations that many students knew they just couldn't have with family or friends. These conversational non-starters were related to topics that are part of the curriculum in both CFS and social work education: social justice, privilege and oppression, and relationships between identity and social group membership and power. Students often described these as political differences or differences in levels of critical consciousness in terms of awareness of privilege and oppression. Below, Amy, a white lower-middle class CFS student, shared her thoughts about conversations with family, which could turn toward conflict easily:

And so, um, as far as that goes, like my friends are very supportive and I don't find any sort of conflict with um, with things that I bring to the table, as far as things that I've learned. But with my family it's different. School is definitely-I was always sort of...liberal-leaning and sort of socialist leaning, but this program [laughs] has just made that so much more intense, because I'm more informed now and I feel more strongly about that. But my family's not, my family is very 
conservative. Like even my_-brothers-who are older than me and, I don't know, it's weird, cause they're kind of...I don't know, they're kind of liberal in their, like, lifestyles? But they're conservative in their thoughts and opinions about things. So I think when I go home to visit-I've lived here for like ten years now, and when I go home to visit, sometimes I wonder, is it even worth it to bring up these things? Because I have so little time to actually spend with them. Do I really want it to be, like, you know...conflicting, conflictual like, dialogue, like arguments, like not really arguments, but just sort of intense conversations, and so, I avoid...talking about some of the things that I learn at school with my family. Even though, I mean, they're super supportive, and super stoked that I'm in school, but, um...I don't know. That's how I usually feel. I'm like, “Oh, I don't want to talk about it." [laughs].

There was a lot happening in this paragraph. Amy noted that she's always had political differences of opinion from family, but that they've been heightened by her program of study. Amy also noted a limit on conversations that several other students speak to: there wasn't enough time with family to fully explain her ideas. Because her time with family was so brief when she did visit, she steered away from potential conflicts, doubting the ability for each party to fully explain their ideas and be understood. Here I was also struck by Amy’s cautious and retreating descriptions of conflict: "conflicting, conflictual...dialogue...arguments...intense conversations." I'd like to explore this further in the discussion in light of the ways we frame conversations and disagreement in the classroom. Amy's de-escalating descriptions of interactions echo middle class values 
around avoiding conflict and may make conversations that involve disagreement difficult in classrooms.

In the same conversation, Lauren expressed a similar desire to have political conversations with family members, who lived much closer than Amy's and with whom she had some ongoing contact. But here, rather than retreating, Amy encouraged Lauren to try to talk with family:

Lauren: Oh! Conversations [both laugh]. I would love to have the conversations that we have, like in Law and Policy, oh my gosh! [all laugh, inaudible comments] I love it, it's so interesting, but-I come from a very conservative family. It's like everything that we talk about is like, so on the other spectrum, and I think it's fascinating, cause I wasn't taught all that, and it's completely eyeopening. And I would love to bring up to my dad, but I know... shit would hit the fan [all laugh]. He would like, destroy me, bring down whatever I'm trying to say, cause he's like, his way or the highway [laughter].

Amy: But you have facts and figures to go with it! Lauren: Yeah, all my...no...

Amy: Just like, sift through your notes while you're having a conversation! Lauren: Actually I'm really tempted to do that. [more laughter] Um, but yeah. So...it would be fun. But it would be dangerous as well.

Here I was struck by the relational weight of Lauren's words: "shit would hit the fan," her father would "destroy" her, and it would be "dangerous." Here both classism and sexism complicate power relationships between Lauren and her father. By virtue of her 
higher education, Lauren knows that she is achieving some measure of social mobility. But her description of her father's possible resistance to her ideas speaks to patriarchal norms that can silence women's voices. Here I'm reminded of the delicate back-and-forth Presser (2005) described in power in research relationships. Even the idea of being prepared with evidence to support her new views was not enough to help Lauren feel confident in political disagreements with her father. It's likely that many students at the same institution will develop more liberal perspectives than their families, based on the institutional culture, regardless of family educational attainment. However, this shift away from family politics may seem especially dramatic for first-generation students in light of all the other ways that their educations were marking them as different from family. For similar reasons Jaclyn, a Native American and white working poor MSW student, expressed frustration at speaking with her parents about current events. However, Jaclyn felt comfortable challenging their views and instead felt more frustrated that her ideas and experiences were simply dismissed:

Like some of the things that I've brought home and talked to them about, they just — they don't understand it. Like, I did a report, um, first term on [local agency serving sexually exploited minors] and was showing them videos and kind of explaining the, the interviews that I got to conduct and my dad was just like, “That stuff doesn't happen." It's like he just doesn't want to believe it and um, so I've just learned that I can't have those conversations with them, because...they're - they don't, they're not gonna process it, and um...it probably [laughs quietly] is just a waste of my time. 
However, Jaclyn noted that she continued to offer ideas and readings to her family, and to her mother in particular. Jaclyn felt that her parents were "pretty naïve to what's going on in the world," but I don't think her parents are an anomaly. It seems that many people are buffered in their daily lives from learning about the lived experiences common to clients served by social workers and other members of the helping professions Dave pointed out another reason why it may be particularly hard for working class families in particular to stay informed of current events, "their interests are in surviving, and paying their bills on time, and having enough food...so they understood that there was more than that, but...they're in a position right now where...they...feel content."

In another conversation between a young white woman and a Latino nontraditional student completing their BSWs, Juli and Arturo discussed how their conversations with family and friends were strained:

Arturo: And now they [brothers] said that I talk differently. And then-'cause my ideas are different. So I don't...care much about what they...they tell me about family problems or...so I can't avoid it.

Juli: And going off that, I think like, language is different for an educated person versus somebody who doesn't have that same education. So sometimes when I'm talking to family about social justice, or...like oppression and privilege, they're like, I have no idea what you're talking about.

Yeah...

Juli: So I feel like there's a, a language barrier in a way?

Arturo: Yeah, yeah especially because- 
Juli: --but you know-[softly]

Arturo: --that's very true what Juli said about, we get kind of impregnate of this language, which is, you know, about social justice and your own community and groups and whole social work language...so, you know, even though I have tried to put myself on the same level as my brothers now...is not, I can't get anymore. And not just the language, the ideas, you know? The thinking, you know. I don't even want to know what, what they tell me about stuff [Arturo smiles and Miranda laughs].

This part of the conversation was interesting because it represented one of the few points in which Juli, a traditional-aged white female student, and Arturo, a non-traditional aged Latino student found points of common connection in their conversation. In this case, social justice and the awareness of oppression and privilege formed "language barriers" in their conversations with family. Juli felt that she was the "black sheep" in the family and had always been somewhat distant in terms of ideas and values. But for Arturo this was a new experience, and he was told by family and friends, "You sound different. You are not the same." "Or the letters I write to my mother," Arturo noted, "It's not the same writing." Much of the rest of their conversation diverged in separate directions, with Arturo speaking about experiences of racism and feeling separated from others in school. This separation from others may have been more painful because Arturo's experiences of the world seemed inherently relational: when he spoke about himself it was more often in the plural "we" than the singular "I" or "me," and he had a clear view of what it meant to be a first-generation student. This struck me during analysis: Brown and Gilligan's 
(1992) voice-centered relational data analysis involves four reads of each transcript, with the second focused on the speaker's sense of self. Brown and Gilligan recommend listening for "I," "me, and "myself." Arturo was much more likely, though, to construct self in relation to others, and his sense of self was almost always the plural "we." Brown and Gilligan's assertion that sense of self is framed in speech focused on the singular individual probably needs to be revised, as residue of Western notions of individualism that clearly didn't fit Arturo's experiences of identity.

In contrast, Juli said she was still figuring out what it meant to be a firstgeneration student and spoke about herself primarily as an individual who needed to get through school on her own. The impacts of sexism and racism also seemed prominent in our conversation, although similar to observations about Lauren's stifled conversations with her father, power here was multi-layered and shifting. Juli, perhaps out of awareness of her privilege as a white person, created space for Arturo to speak about experiences of racism that led to his persistent feelings of exclusion. Another interpretation, though, could highlight Arturo's privilege as a male speaker, which allowed him to feel comfortable speaking first and directing the conversation. In this interpretation it's important to note Juli's complicity in self-silencing (Juli's tentative and quiet "but you know" was quickly subsumed by Arturo's interpretation of Juli's earlier statement in the excerpt above). Here too notions of power as contextual and subjective (Collins, 2000) are helpful in examining the relationships each speaker holds to privilege and oppression. In listening to Arturo and Juli it was plain that first-generation student experiences are by no means universal: age, gender, race, (and correspondingly, ageism, sexism, and racism) 
and one's own level of reflection on what it means to be first-generation are powerful influences.

Despite the difficulties connecting with family in conversation, many students continued to reach out to them. This excerpt is important to quote at length to capture the push and pull between Maria, a working class Latina completing her BSW, and her family. Maria lit up when talking about her advocacy and learning about feminist theory, but described a family response which had become routine when she shared new ideas about gender roles or child discipline or white privilege and experiences of oppression: “Oh Maria! That's your social work stuff!"

Maria: I'm learning about all these things, and like, policies and macro-level work and like, all this great stuff that I totally love! But um, so when I go home, of course, you know, I'm very close to my family so I want to share these things. But when I go home and I'm like, you know, and I'm like trying to explain or like, share, you know, white privilege and oppression and that and they're like, “Okay...so what?" ... so I guess maybe I throw too much at them at once so it's like, "You know, Maria..." they probably block me out and that's probably my fault. But sometimes I wanna have these conversations and sometimes I can't but I always tell them and they're like - you know, like gender roles and all that stuff, you know. Or I did like, lobbying in Washington D.C. and so I was involved with that. So when I bring back all these things and I tell my family about it, it's like, that's when they're like, “Oh [hint of disgust or disinterest], Maria. Oh, her 'social work stuff'.” Or even like, I've done--so I've been doing an internship 
working with children, so I tell them about, like discipline and positive behavior and like all of that stuff, and they're like, "Ugh! Maria."

Yeah...[sadly]

Maria: Like, "No.” You know? "Like, we're gonna...do it like, the old style, right? Like how we do it." Like, no, a lot of "no's" and a spank here and there, and... and so when I bring up these new ideas, that's when they're like, “Oh, you know. Here she comes again. Oh, Maria-Maria and her social work" or-oh, I don't even know what they say [confessional tone here]. But they just, they saythose answers are the ones I get, I guess come up a lot when I start sharing my ideas on certain things, I guess. Especially when I-but gender roles, that's the biggest one right now because I-I mean, I just got married and then my husband is very...traditional, um Mexican male, so that has been really challenging.

Yeah, yeah.

Maria: And so I think that's the biggest one that they all say, "Ugh! Her 'social work stuff'."

So your social work-your "social work stuff," whatever falls under that, um, umbrella, I guess. I mean, it's, it's big.

Maria: Yeah.

Values around parenting, and like talking about oppression, and recognizing white privilege and discrimination, um, and it's-it sounds like, that's a lot-a mindset shift or a values shift-

Maria: --mm hmm- 
RELATIONAL WORLDS OF FIRST-GEN STUDENTS

--that's asking family to do a lot, that that's the kind of, "Ugh, here she goes again."

Maria: Yeah. And I think a lot of that, because, you know, specifically, they've experienced discrimination, you know? I mean, my husband, you know, all of that — getting paid, like, way less than he's supposed to, and all of these things. So it's when I say that, that's like, "You know, that's never gonna change." So it's kind of like, they've lost hope. And so when I come over here, like, "Maybe we could try this, or maybe if this happened, you know?" They're like, “That's never gonna change." So I think it comes from like, you know, they lost hope or they, they don't think it's ever gonna change, so what, like why should we ever get involved in attending like, any community forums. Like, immigration reform, that was a big one. And it's like, encouraging that and saying, "Oh, maybe if we can all get together-“ it's like, "Well, we've done that, and nothing has changed.” So, so, you know, it's like, lost hopes and so here I am, also, like, kind of pushing and telling them what we could be doing, and so...

Yeah. So part of your "social work stuff," is like that positive vision, too?

Maria: Mm hmm.

Like, for change, and-

Maria: --yeah!

Notice the pressures from both sides: both Maria and her family were pushing at each other. Maria conveyed her family's “Oh Maria!” in tones that varied but suggested exasperation, disgust, and dismissal. Maria had not given up on talking with her family, 
even about subjects that were deeply values-based and provoked strong reactions, around culture and gender and ideas about child discipline. Maria also noted that it was not her family's lack of awareness that prevents these conversations. Maria's family members were well aware of oppressions because they were woven into the daily fabric of their lives through experiences of racism and nativism. Maria suspected the "Oh Maria!" dismissal might have been the way that her family members guarded against getting too hopeful. In another part of our part of the conversation, Maria declared that the cultures of home and school would always be separate for first-generation college students. She attributed part of this separation to the same "language barriers" Arturo and Juli spoke of, which are primarily conceptual. However, in this case Maria highlighted the additional language barriers in her communication with family members, "When I go home it's in Spanish and so it-everything changes in the language, you know?"

Students described experiences of stumbling into potentially difficult conversations when family members or friends used certain words that signaled a difference of opinion. Bob, a working class Latino CFS student, described “...talking to my friend on the phone, and they were using words that - I wouldn't use, at all..." Lainey, a working class MSW student who emigrated from China as a child, described her frustration at her parents when their biases toward other groups of people based on race or experiences of homelessness emerged. She made sense of this in light of her parents' experiences as immigrants:

...moving here from another country and to have to make a living for yourself, they survived a lot...they have a certain concept about who they [groups they 
RELATIONAL WORLDS OF FIRST-GEN STUDENTS

have biases against] must be. Mostly stemming from survival and fear. And so, when I tell them...”Really?" [ raises one eyebrow and her tone here suggests skepticism, followed by a laugh] Maybe, um...when I'm bothered, I kind of push back a little bit, you know, it, it may not always be well received.

While a diversity of opinions are encouraged in the classroom, it is generally not considered professional for students pursuing a degree in CFS or for students becoming social workers to express biases against people based on social identity groups. For these students, the emergence of family biases often signaled a need to interrupt oppression, but most students struggled with how to articulate the problems with these biases to family. Interrupting family member's biases was also complicated by experiences that had driven family members to form biases. For Lainey and Maria, their family members may have held biases against certain people borne out of experiences that taught them they couldn't trust members of those groups.

At other times students who had grown to feel comfortable with complex conversations in school tried to have these with family and realized distances in ideas, values, and comfort with deeply charged topics. Clara, a white lower-middle class MSW student, described her unease at realizing points of disagreement with family, particularly when discussing current race relations:

I can say a lot of things here, and I can...generally feel like I'm gonna be understood, or at least I'll get some clarifying questions that make sense to me. But I can say the same thing at home, and get like, a weird look, or like a concerning look, or just like some clarifying questions that are just like, "wow, we 
RELATIONAL WORLDS OF FIRST-GEN STUDENTS

are totally not on the same page with this" Like, maybe that was a mistake to say that out loud.

Clara's age and gender were probably salient in a story she told to clarify her words above, when she attempted to discuss the grand jury's failure to indict Officer Darren Wilson in the shooting death of Michael Brown in Ferguson, Missouri. Shortly after the non-indictment, she visited family in Missouri and brought the event up with her uncle, a retired police officer who picked her up from the airport:

I've definitely made the mistake of saying something that I thought was pretty neutral, [pauses as everyone laughs], but then like realizing...it became me sitting there, like, and we had a long drive from the airport, so it was just like sitting there like "When... are we gonna get back to the house" because obviously he's not hearing a word I'm saying, he's just talking... maybe I have to be like a lot more intentional, like I can--I don't have as much room for slipping or messing up when I'm around family cause it's like, it's limited times ...it's kind of setting the stage for what they think I think [others "uh hmm” in agreement]. I don't have more time to kind of, clarify [ "uh hmms" in agreement]...

Notice here that Clara pointed to the same time strain that Amy felt: when time with family is limited, it's difficult to fully unpack complicated ideas about sensitive topics. Even discussions of new ideas that don't carry deep political consequences could be difficult. Davis, a white low-income male finishing his degree in CFS, talked about sharing new things with family here, but stopped short at describing family as enthusiastic about the things he was learning: 
RELATIONAL WORLDS OF FIRST-GEN STUDENTS

Davis: I think that part, I think that's where you find some of the encouragement too. Because, when you're talking with your family - or friends - about what you've learned in school, that's where, I think, it comes through for me, they're like "That's cool," and it's different from our experience before where it was just kind of normal social interaction-yeah.

Davis: You know, daily activities.

So it sounds like you're saying, like, having these conversations that we have in the classroom, like, that's welcome in my family. That's interesting. Davis:... Yeah....I don't know [smiles]. Maybe I shouldn't go that far... Some of the topics might still be...kind of challenging, I guess.

\section{Responses to communication breakdowns.}

Students had varying responses to the experiences of being limited in conversations with family and friends. Some students felt comfortable leaving those conversations alone. In the passages above, Amy seemed comfortable with limiting conversations with family for the sake of peace, a position that several other students held as well. Dave, the MSW student who expressed an acute sensitivity to the potential for feelings of power imbalances between him and his family, noted that there are "multiple truths" and said "I guess I feel pretty comfortable with where I'm at and, you know, with where they're at."

However, the inability to express ideas fully in conversations with family and friends presented deeper emotional and relational challenges for other students. Here 
Brown and Gilligan's (1992) ideas about women and relationships seemed relevant: women were more likely to express sadness and loss over these breakdowns in conversations. Lauren, the white working class student who shared her desires and fears about discussing policy with her "conservative" father, said this about her inability to share the experience of having her "eyes opened" in conversations with family:

Lauren: [It] sucks because, like the stuff that we're learning, I love it, it's part of me. And I feel like if I can't share part of me with my family...they kind of don't know me, and I don't like that. [pauses, and then continues with slight sadness, group is quieter] ...I feel like at least at work and at school, I see people every day so they know me. I feel like they know me better than my parents and my sisters know me, and I don't like that...So that's, it's hard to be open, but...[pause for about 5 seconds] is that good? [to group, quietly]

Bob: Yes [quietly].

Here Lauren's admission that she felt like her family didn't know her suddenly quieted the conversation between the four students. The joking and laughter that had been part of imagining a heated debate between Lauren and her father was instantly replaced by stillness and suddenly Lauren's voice was central as she spoke to truths that might have captured other students' feelings of being unknown. Lauren's sadness at slipping further away from family hinted at the "cultural suicide" Tierney (1999) argued students of color faced when asked to separate from home communities. Lauren's embrace of learning and identification with her studies could be read as a measure of successful integration, but her deep sadness at what may be lost deserves attention. Here there was another moment 
of connection between students, as Lauren's “is that good?" was answered by another student, whose soft tone matched hers: "Yes."

In another conversation, three young white women pursuing their MSWs took turns describing what it felt like to not be able to speak freely in conversations with family:

How does that feel, that, that distance, or that feeling of, "Okay, these are conversations we can't have"... Like, how would folks describe that?

Clara: For me it's isolating. It's like not being understood. Like all my mother could see when I was being a part of the protests and stuff, was that I was in danger. And she had no awareness of what the cause was, what I'm standing up for, and if I am in danger, then why am I choosing to put myself there? It's not for fun. I'm really making a statement; I'm being with like-minded people. So it feels kind of like...yeah, I think isolating.

Nancy: The word that was coming up for me was disconnected. Because I feel pretty similarly about that. Like especially with my siblings. Like, I don't feel a connection with them around a lot of things, because I do have that different perspective, from being in social work specifically, ...but also, I feel like they don't come--they don't necessarily see me the same as they did before, before we all, kind of, picked our future routes and so we just don't connect about the same things we used to, even if we have the same things in common now. It's just kind of ignored, especially 'cause I have nephews, and I really want to have more of a connection with them, but like my stepsister has told me, "Okay, don't talk to my 
nephew-kids about college. Don't talk to my kids about - like, different things" And they're only - today's their birthday, they're five. [laugher] They're twins. And so it's just kind of interesting to me that it's like, you can have this relationship with them, but it's gonna be really specific. And they told me they don't want their kids to hear my liberalism. [pause, some silence in response]...

Michelle: Yeah, I mean, definitely I feel the same way, the same things that you guys have described, the isolation and the disconnection. I also, the word that was coming to mind for me was like, just, I feel fake, like I have to put on this like - I have to be someone I'm not, or like, an edited version of myself, in order to make it through a conversation [laughs]. And I also, I think that, like I experience a lot of guilt sometimes too, and blame...for like, leaving family behind, quote unquote. Like I have a cousin who is older than me and she constantly compares herself to me and so like I feel really...that's not my intention. [laughs] It's kind of a crappy way to feel.

Here each of these women's words painted a slightly different picture of the experience of being limited in conversations with family. Conversations felt limited for different reasons: Clara's parents didn't understand her activism and seemed to respond from fear and genuine lack of understanding. Nancy's family members explicitly limited her conversational topics and set clear boundaries with how she could interact, especially with young family members. For Michelle, it was less clear why she felt the need to censor her words, but she felt like she couldn't be authentic with family. Similar to other parts of conversations, this moment stood out as a rare space where each of them could 
explore and describe the sense of being unable to be fully themselves in conversations with family. Each woman described her perspective on a shared experience, one they may not have been able to articulate in other spaces. As an observer I was moved by the relationship that was formed, even briefly, over this question as each woman listened to the other and then added her own spin on the experience.

While some students felt a sense of sadness and being unknown or not fully authentic in relationships with family, a few students described experiences of outright rejection. Nancy, a white working class student completing her MSW, noted that her mother and stepdad supported her, but that:

None of my cousins, and my aunts and uncles, they didn't, they didn't--they barely finished high school, and some of them didn't, didn't do that at all, and so like, at big family events and stuff, I get kind of like, almost ignored and treated, um...I've been told, "Oh, you're such a snob because you went to school." "Where'd that come from?" But it's definitely something that comes up a lot, and so I don't really have relationships where, like, very strong relationships with a lot of my cousins, because, like I'm viewed as very different. And that's the same with, like, my step-siblings as well.

While Nancy questioned why some family rejected her, she also recognized some origins of the conflict, often arising from casual conversations. Nancy described how her values and ideas have been received in the relationship. 
RELATIONAL WORLDS OF FIRST-GEN STUDENTS

Nancy:...I've said things like, like, one of my aunts gave my nephews something that had a little bit of pink on it, and my stepsister was like, "Well, they're never wearing that." And I was like, "Well, why not?" and it kind of sparked a problem. And so it's just interesting to hear. And it's also really frustrating because I want

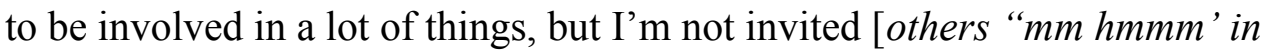
agreement]

With family?

Nancy: Yeah. Like, they live 25 minutes from me, they live out in the [nearby rural town] area, and in order for me to go to things, I have to hear through my parents. They don't invite me directly. And yeah that may--at first I thought, “oh well, it's because we're stepsiblings," but then like, when my brother's in town, they invite him to everything. And I think it's because maybe they just like, relate more. I don't know.

Nancy shared that one of her siblings refers to her as "the commie" and that she's been told that she's "too political," something other students have been told as well. Maria, a working class Latina and BSW student, hinted at her family's potential rejection of her because their Catholicism dictated disapproval of same-sex relationships. "I used to date a...a girl," Maria shared. "And when that ended it was kind of a relief for everybody that, you know, I went on and married a man." Maria challenged her family’s lack of inclusion, but shared that "they kind of don't like me for that." Another student told a story about his father issuing a more ambiguous reaction to his ideas during his first visit home as an undergraduate student, "I came home and I made some statements and he said 
'Oh, I can tell you've been to college."' Dave said that these words "still resonate[d]" with him eight years later.

\section{Working to Stay Integrated in Home Cultures}

The dominance of ideas about social and academic integration as the primary goals for first-generation students has led to important explorations of the work these students must do to learn to master the "student role," for example (Collier \& Morgan, 2008). But while these students were working to become integrated in school they were also working to stay integrated in home cultures. It's also important to emphasize who was doing the work to maintain connections: students described several strategies they employed to remain relevant, but did not share any strategies family members might be using to stay connected to them.

\section{Code switching and self-silencing.}

Because conversations were often a site of tension between students and families, and because students seemed to take sole responsibility to bridge the potential distance between home and school, it made sense that many students modified and carefully tailored their words to maintain connections to family. Avoiding certain topics of discussion, code switching, and making judicious decisions about sharing information for the purpose of educating family members were several ways students worked to stay connected to families. These tactics were more common among students pursuing degrees in social work. Juli, the BSW student who shared her struggle (along with her 
classmate, Arturo) of trying to remain relevant to family, described how she adapted to the different cultures of school and home:

Juli: I guess I don't feel like I am one person here and a different person here. I think I just, like some of my-like my language is one thing that I'm thinking of particularly. Like I speak one way when I'm interacting at school, but then when I'm at home, like I'm a lot less careful about what I say. I try to use language that more so the people I'm with will relate to.

Yeah. Can you give examples of that?

Juli: Um. [quietly] That's a good question...I think...like maybe cursing is like one example of just like, my family dynamic, like that's kind of one way to show that you're a part of it. But then when you're at school, sometimes that doesn't feel appropriate? So I try to throw in more curse words when I'm at home to show that I'm still want to be a part of this...family, and like, relate to you guys. And I don't want to be-I don't want you to think I'm too good now... or whatever. I don't know, can you relate to that? [to Arturo]

Arturo: Yeah because-- we tried to a...you know, we talk about things sometimes, we have...so I get to school and I talk to some person, you know. Then I go to the place I live, which is a low-income housing, where a lot of--most of the people there, addicts and all stuff. So I had to put myself in kind of...cause I interact with them, you know, we change stuff. But even then...they noticing that. The other lady one time went "Arturo, you are different now."

\section{Mmmm.}


RELATIONAL WORLDS OF FIRST-GEN STUDENTS

Arturo: So they, you know, even though I tried to put myself on the same level...it's, it's not easy stuff. But we change. Too much. Too much. Anyway... Juli: Yeah. But that's totally true, too, in like, I'm trying to think of like calling out, like jokes that are not funny-

--right-

Juli: -- or like using words that are like, I'm not okay with that. So not laughing, calling it out, that immediately puts you back to being, like, you' re...like 'Ugh. There she goes again."

Right.

Juli: Or something like, totally excludes you.

I was struck by Juli's words above: "I still want to be part of this family...I don't want you to think I'm too good for you." Using "curse words" was a subtle way that Juli signaled her continued interest in remaining a part of the family. Juli questioned the appropriateness of using these words at school, and I thought this reflected her genuine uncertainty about the rules guiding classroom dialogue. The truth is, different instructors have different levels of comfort about using "curse words," and it may be that the ambiguity regarding the appropriateness of these words is especially unclear for firstgeneration students who are working to read the unwritten rules of the academy. This lack of clarity exemplifies the "hidden curriculum" (Giroux, 2001) and functions to maintain inequalities between students who come armed with knowledge about social roles, including when they can speak freely and when they cannot. Juli has acknowledged 
her privilege as a white person, but is also at a disadvantage in her uncertainty about rules which have never been made plain to her.

In another part of the conversation Juli also shared that she adapted at school and felt comfortable around other first-generation students, but tried to "sit up straight" and "not be, like, sleeping in class" around students who seemed "more educated in social roles." This is another nod to the notion of embodied forms of cultural capital (Boudieu, 1986). I would argue that Juli is educated in social roles, but the question of whose roles are granted more currency in higher education is not always honestly and clearly addressed.

Arturo struggled with the question of how much of his education to share with his brothers, noting that he, as well as his brothers, was once socialized to expect and see oppression as a normal part of life as a "member of a minority group":

Arturo: I--most of my life have been oppressed. And I was kind of unconscious of that. So I understand when I talk with my brothers or people, it's still, they haven't, they're not...they don't know much about this stuff. And I'm thinking it was probably, it was better not to know anything. And now that I know things... it's like... this anger about stuff, you know, and I say, "Well, probably they are better that way. Why you tell this stuff?" They'll never understand, you know. You-do you, you're saying, Arturo, maybe — my family is better that way, better not knowing?

Arturo: No, I don't, no, and...probably. I tried to tell them, I would like to not tell them. Because...I think is one of my...jobs...to educate people so they can have a 
RELATIONAL WORLDS OF FIRST-GEN STUDENTS

conscience?

Yeah...

Arturo: But I know they think, when I say, like well, they...they don't feel like I'm feeling. You know?

Mm hmmm. I think I do. [laugh, quickly] I think I do very much.

Here Arturo considered the possibility of not saying anything to his brothers about his understanding of oppression, but eventually decided it was his job to educate others. Here Arturo's thoughtful attention to educating family members was reminiscent of Maria's attention to educating family, even in the face of being dismissed ("Oh Maria! That's your social work stuff.”). Maria described how she considered her family members' experiences, personalities, and interests before deciding which ideas to share.

Maria: So I used to share. When I started my associate's I would like tell them all about it and what I was learning, and you know, not so much of it, I guess. Because, I don't know how to say it, because I do share stuff at home, so you know stuff about maybe, my niece and I share something about, you know a development milestone?

Yeah.

Maria: So I share stuff like that, but I wouldn't say like I get more into a like a deeper conversation perhaps, where we're talking about, you know, history. I don't get into that - a whole lot of that stuff. But I do share certain things...my husband didn't grow up here, he didn't go to school here, so he didn't know about who Cesar Chavez was, so I shared that, you know? ... So it's kind of like, since I 
RELATIONAL WORLDS OF FIRST-GEN STUDENTS

know them, my husband he's like more...he's experienced a lot of discrimination and stuff... So I knew that that would probably catch his attention and stuff, you know? So I tell him in little pieces about that. But then like, children—child discipline, I'm finding that I didn't, you know, share a lot of that stuff because he has a very different —um, thinking regarding that? So it's like kind of their personality and who I can share what with, you know?

Maria followed up this explanation with an additional point to consider: students are afraid of presenting themselves as knowing too much. Here she explained why she downplayed her knowledge around friends and family: "I don't want to appear as bragging...I think a lot of the times that's why I don't say anything, because I don't want them to be like, 'Oh, just because she's in school,' you know? 'And she already thinks she's all that."' Maria's concerns about bragging were echoed in the concerns of other students as well. Veronica, a Mexican American student completing her BSW spoke at length about being seen as “an information person: I don't want to feel like I'm like this information person. Like, I know everything, I know all the answers.” This was not a simple position for Veronica, though. She also spoke about feeling stuck between "many different feelings," because she was grateful for her education and didn't want to be selfish with her knowledge. But gender and cultural influences made Veronica nervous about appearing "stubborn," or like she "knows it all." Dave, a white, working-class MSW student, was conscious of having had an "air of being cocky," in the past as a student, or acting "as if I know better." He gauged his conversations with parents carefully, paying attention to how long he spoke without any responses from them: a 
period of silence indicated he'd probably plunged into a conversation that wasn't equally enjoyable for all parties involved:

And I don't want to come across as... kind of uppity, or um... whatnot. So I'm conscientious of that. Um, and like I said, when I start getting, like, too deep into what I'm doing in classes, I kind of have to check myself and I can see that they're, or hear that they're kinda distancing or shutting down.

As noted above, the majority of the excerpts in this section came from conversations with social work students. Students pursuing a degree in CFS also spoke about limiting their selves in conversations, although this was mentioned less frequently. The reader may recall Amy, who noted above that there were some conversations she would choose not to have with family. Often their adaptations to maintain connections with family were more focused on self-presentation. I'll return to that in a moment when I discuss the different ways students present themselves in different contexts.

While the students above described self-censoring their words, some students were explicitly limited by family members' requests about avoiding certain conversations. Nancy, another MSW student, explained that multiple family members had told her that some topics were off-limits, "actually I've been told by my parents not to talk about things. Like, 'Oh, they don't understand, so just don't talk about it." This was part of Nancy's sense of disconnection from family: being told not to discuss things like college or her "liberalism" with her nephews. As Van Galen (2014) has pointed out, family resistance to changes in first-generation students is often seen as a barrier to student success. Families are presumed to be "holding students back." However, Van 
Galen highlighted the role of elitism in family members' responses. If family members have encountered judgment or classist responses from other people who have a college education, they may be working to preserve relationships with their children by rejecting changes or words that suggest that students are taking on these values. If this is true, limiting conversations may not be an act of rejection, but of self-protection and even an attempt to keep students connected to family. Dave's father's “Oh, I can see you've been to college" may have been a way to draw attention to Dave's shift in attitudes and ideas, and it worked: Dave was very careful not to be seen as "uppity" and sensitive to power differences his education might create.

Here Bob and Brandi discussed the potential for conflict in conversations with friends. I wrote earlier about Bob's family's general support of his schooling and even his dad's warm envy of Bob's learning. But his friends, from whom he was separated geographically, hadn't gone to college, and would probably have negative attitudes about his ideas, because college wasn't really "the way they were....feeling." Bob noted that he had to be cautious about sharing certain ideas with friends:

Bob: Yeah, I'm gonna have to like, [laughs] you know, try not to talk about it so much.

...can you, like, say more about the negative-what do you think the negative parts are?

Bob: ...the best way I can describe this, I guess, is like a lot of the ideas that I got from college, or a lot of the ideas that I learn about, like just a general philosophy, like feminism or all those ideas, are not going to be things that like, people want 
to hear there.

Gotcha. Gotcha. So the ideas that you have might not be well-received?

Bob: Yeah. Yeah.

Gotcha.

Lauren: Is it that they don't like the program that you're in, or is it the fact that you're in college?

Bob: Well, it's as much not as much that they don't like the program, they understand what I'm doing, like "Oh, it's cool," but it's like...it's like they're used to what they're, where they are at right now. All the things I bring to the table may seem...either pretentious, to them, or like I'm talking down, or like I'm not going to be at the same...pace as them. I think they're anticipating that, like when I come and see them...like, come in with all these ideas and like, very liberal--

Lauren: --yeah--

Bob:--and they're like, “We don't care!” So yeah. I guess, does it answer your question? I'm sorry. [laughs] Yeah, you said specifically, like, I like feminist ideals and things like that. Yeah? Bob: Yeah. The other day I was just talking to my friend on the phone, and they were using words that - I wouldn't use, at all, but I was just have to like...if I were even to complain about that, they'd be like "Oh, like who do you think you are?" I'm just going to have to accept, have some acceptance, and not try to be like that person to come in and rescue them from their, their Southern, like, backwards ways. It's not my position. 
Right. Other thoughts?

Brandi: Well, to-I would probably agree with...Bob. Maybe not as negative. But I would say, like with my friends, like how we feel like, we might downplay things. Most of my friends are, I mean they're...not-I wouldn't use the same analogy as being negative, or same word "negative," as Bob did, but almost everything I would "ditto" what he said. Um, I do think most of my friends are pretty much just not interested in...like, whatever. And I know that, and so I don't really_-you know, I mean one of my friends started going to college recently, and we've been friends for a long time so we have great discussions, but...because she's living it now, we do get to talk about all these wonderful things. But all my other friends, they haven't been to college, and...they're not-I mean, our discour conversations are really different than the conversations I have with my peers here at PSU. And it just is, and I just accept that and know that they don't want to hear about my...I'm too busy 'cause of finals. I might just say, "I'm too busy." Like, they don't_-you know, and it makes sense because before I was in college, I didn't much understand or I couldn't, or wouldn't understand what people meant if they were doing college.

Here several of Bob's ideas struck me as important. He was cautious about how his friends would react in conversations where his beliefs emerge: he could be seen as "pretentious," "liberal," or "talking down" to his friends. Bob's sensitivity to both social class and regionally based stereotypes was evident here, as he refused to be the person that would try to "save them" from their "Southern" or "backwards" ways. In contrast, 
Brandi felt the need to temper herself in conversations with friends, but this was largely because friends didn't have the experiential context for understanding why she was so busy.

\section{Navigating between worlds.}

For some students, there was a clear sense of moving back and forth between two different "worlds" of school and home. Sometimes this was an occasional journey, such as when Clara, a white, lower-middle class MSW student traveled home to see family in another state. She used the geographical distance to "switch gears" to prepare herself for conversations at home, knowing that she won't be as easily and readily understood at home as she is at school. Lainey, a working to middle class Asian American student pursuing her MSW, explained that her identity as an immigrant was central to her experiences as a first-generation student. However, this also meant additional layers of separation between home and school, which included not only concepts and ideas important to social work education, but differences in conversational pragmatics between the different languages spoken in school and at home:

And it's not like my parents don't care. And they've heard a lot about college. [Miranda laughs in response to Lainey's expression and tone here]. They've heard a lot about college. Um, but what exactly am I doing in college? Writing papers, um, how to negotiate and navigate through systems and how do you work with teachers, your peers...yeah, I feel like I've had to kind of...just figure it out by myself. And sometimes it can be a little lonely. That at the dinner table, like "big stuff happened at school," but I am not really sure how to convey why it's a 
big deal, why it's interesting, and what it means, so I don't know if you've [Jaclyn, her conversational partner] had that experience but...

Lainey described feeling lonely at home because she couldn't convey her experiences to her parents. Lainey explained the additional challenge of trying to transfer the social work skills she's practicing to conversations with her parents, who speak Cantonese: “How long do you actively listen before you interject?" This was, of course, dictated by the pragmatics of language, which vary widely. The open, undirected conversation which heavily emphasized listening that Lainey was learning as a social worker felt awkward and inappropriate in conversations with her parents.

In another exchange, Lainey highlighted a crucial understanding for identity and relationships: she felt like a different person at home than she was at school because her parents treated her differently at home than people at school treated her:

Yeah. There are two different lives. I-I'm different in my school life than when I'm at home. I mean, of course I'm not a completely different person! There are certain things that I just, um...I just do differently. Even just, I'm-I'm working to... sew these two worlds together more actively now that my parents - my family's going through crisis. But the way that I listen...in the "school/career" side is different than in my family. Some people...here my colleagues may see me as someone who's patient, who can listen active-- "active listening" and "motivational interviewing" [smiles and laughs, Miranda laughs in response] you know, all those skills that I can actually actively use at home it just...doesn't show up the same in - they have a different idea about who I am and what my 
RELATIONAL WORLDS OF FIRST-GEN STUDENTS

capacities are based on their knowledge of me growing up. But now that I'm at this point in life and with my education, I'm actually a different person than what-who they think I am. And so when I interact with them, sometimes I—feed that back into them. You know, what they expect-I give them what they expect. But lately, I'm doing - I'm working on that. I'm working on sewing these two together, like I said. But I've noticed that in the past it's been that way.

Here Lainey highlighted the co-construction of identity (Gergen, 2009): "they have a different idea about who I am" and "I—feed that back into them...I give them what they expect." Lainey also spoke plainly of being in two different worlds, which she moved between each day, living with parents and traveling to school each day. Jaclyn, a Native American and white working poor MSW student agreed with Lainey's assessment of different relational contexts and the power they have over identity:

A lot of what you said is identical to me. I think it's-it's a whole 'nother world, I think we live a whole 'nother world from our family and it's - especially with my undergrad but also with my first couple terms here in this program, I would go home and I would realize that I was a different person at home. And I didn't necessarily like that person, um, and not that I don't like my family. I've grown to accept them as who they are, which was really something that was challenging to me for a while. Um, so I have now grown to accept them for who they are but I did notice when I would go home and visit I...I was a different person. I was judgey, and I was talking in their, in their lingo, and it was like, I really started to reflect on my childhood and how unhealthy I was raised — and not that they are 
bad parents or that they did anything wrong, but-- being a social worker is so powerful it really gives you whole other life - a whole different life to live. It allows you to see things in different perspectives, and put yourself in other people's shoes who maybe weren't as privileged as even I was, and I wasn't very privileged.

Like many students, both Lainey and Jaclyn felt like they were the most authentic at school. For many students knowledge and skills brought a sense of pride. But there was also a sense of questioning of authenticity when they described playing different roles. At one point in this conversation Lainey's voice lowered and she confessed, “Sometimes I wonder, I'm like, 'Ugh! I’m kind of wearing different masks!' [expresses disgust or concern] 'I'm kind of fake!' [laughs]” Jaclyn replied with a whisper, “I feel the same way." Another MSW student, Michelle, explained that she felt "fake" because "I have to be an edited version of myself to make it through a conversation." "The conversations are very basic with certain family members," she noted. "'How are things?' 'Oh, your garden's doing well, that's good.'” Here Michelle quipped about the lightness of these conversations that stay at the level of small talk. In contrast, there was a sense of urgency in Lizette's description of avoiding criticism of certain family members through limited conversations ("How are you?" "Fine" "How are the kids?" "Good" and I'm like, “Anything else?" and they're like, "No." "Okay). But for both women, conversations with some family members had to stay superficial in order to stay in relationship, and authenticity might be sacrificed.

\section{Self in relationship: Careful self-presentation.}


RELATIONAL WORLDS OF FIRST-GEN STUDENTS

Above I wrote about Bob's preparation for conversations he could not have with friends. Here Lauren's description of the need to consider her self-presentation with clients impelled him to consider the importance of his appearance when he visited these friends in the near future. Lauren described the attention she gave to self-presentation as she moved between her current employment as a checker in an "upper class town" and as an intern with the Department of Human Services who often worked with families in her hometown, which she described as a "lower class town." In one space she was careful to highlight her work history in order to be respected. In the other space she worked to "bring it down to their level" with the families she worked with.

Lauren: Yeah, because of the reactions I've gotten from people [in the "upper class" town], whenever people do ask me what I do, I'm like “Oh, I'm a front-end manager for Hagen's, which I've been at for nine years." Like, I feel that I have to tell them that I've been there for a long time--

--oh, yeah-

Lauren: -- to like, bump me up. Or there still gonna be like, "Oh, that's cool— anyway." [laughs quietly]

A few moments later, the conversation returned to the different ways that family and community members respond to students, and Lauren described how her goal of being a social worker was valued in the upper class town that she worked in as a checker, but how in her "lower class" hometown, there was a "terribly bad stigma toward social workers." I'll return to this conversation when I discuss implications for socialization into 
the professions, but here it's important to highlight Lauren's different ways that she presented herself.

So there's kind of a tension you have to walk between two different worlds, in addition to school [laughs], which may be another world.

Lauren: Yeah, it's a lot. Yeah, and I feel like this sounds weird, but doing it, it makes sense to me. Like when I'm at DHS, I try not to dress or look so...makeupey and whatever cause I feel like they already have a bad, you know, interpretation of what I do, so maybe if I just bring it down...to their level or something, they'll behave differently and be more understanding.

So this is like, dressing that you do, with families, going to work with families-Lauren: -- mm hmmm--and be like, trying to minimize whatever perceived differencesLauren: --yeah.

While Lauren emphasized her long work history to "bump" herself up among folks in the upper class town she worked in, she had to try to "lower" herself in her work with families she served as an intern with the Department of Human Services. This work primarily involved careful attention to her appearance, particularly clothing and makeup. Here I was struck by Lauren's attention to others' ideas about her in the differently classed worlds she was working in. It also doesn't escape my notice that Lauren's hometown was the "lower class town" and the families she was serving might have felt more familiar, but also expressed a sense of resentment or rejection of her that she 
worked to counter with her appearance. I'll return to this in the section of the findings focused on professional socialization.

Lauren's description of how she chose clothing that would minimize differences between her and her clients prompted a similar reflection for Bob:

Bob: Um, like you were saying, about dressing differently, that's a big-I'd never thought about that and I might - I probably have to do that, when I go back to Georgia and that kind of thing. I just can't, like, look too fancy, 'cause then it's like-

Brandi: --you never look too fancy! [laughter]

Bob: Oh yeah. I mean, like buttoned up and whatever, and it would be like, “Oh! I'm sorry." [others laugh, Bob laughs - hard to hear comments about "A collar!" and "I shouldn't have worn my suit and tie!" among laughter]

Here Bob's realization that he would need to attend to his appearance was sparked by Lauren's description of work that she does consciously. For Bob this seemed like a new revelation. But I was also struck by the subtle differences in their motivations. Lauren expressed sadness that the families she works with have a "terribly bad stigma" towards social workers and wanted to "bring it down to their level" to earn trust and work with families. In contrast Bob, as discussed above, was concerned about appearing "pretentious" and "liberal." It did not seem that he wanted to change the minds of his friends. Instead, Bob seemed primarily concerned about alienating friends and losing relationships. I also feel compelled to mention the relationships that were forming in the room at this point, slightly over an hour into the conversation. It was clear here that the 
students felt familiar with each other, as in Brandi's teasing response to Bob: "You never look too fancy!" For a moment, everyone was laughing at the potentials for awkwardness in appearing "too good" for familiar friends, suggesting that Bob and Lauren were not the only ones who realized how their education could "show up" in their personal appearance. I'll return to related parts of this conversation in the discussion of the implications for professional socialization, but before I leave this it also feels important to highlight social class, particularly internalized class biases students may hold against themselves and those they deem as being from similar backgrounds. As highlighted before, Lauren noted that others in her "lower class town" expressed resentment or rejection of her as a college student and thought this might be because they didn't want to "get themselves out" of the "lower class" town. Below I'll also discuss students" descriptions of higher education as a step up and away from their families and communities that make me question the ways that framing college as a step up may relationally injure students or reinforce internalized oppressions about class and race.

While I will return to this conversation in the discussion, it's important to dwell for a moment on the implications of the work students do to maintain connections to family. As described above, students limit their conversations and engage in codeswitching in order to continue talking with their families. Several students describe downplaying what they know in discussions with family and friends. A few students spoke of putting on different masks or shifting into different roles - even feeling like different people - between school and home. These findings suggest the dominant 
approaches to supporting first-generation students in integration in school are misguided, or at the very least, overlook the relational burdens for students.

At a quick glance, we can see that these attempts to maintain connections require more work for students, who may not be dealing simply with educating their families in jargon, concepts, and assumptions that are field-specific, but who are also met with deep resistance. There's also the burden that comes from relationship strain, an added weight for students who are often pursuing school largely on their own. Students' descriptions of wearing different masks or being different people have important implications, particularly in social work education, which demands that students identify within a profession (Wiles, 2013). Finally, there were tensions between home cultures and school cultures around the value of sharing knowledge and being seen as a knowledgeable person. There's also the question of whose knowledge is more valued in higher education. In the discussion between three white lower-middle and working class MSW students, Clara told a story about her grandfather's farm and the different foods he grew and noted, “...there's so much value on a formal education. And there's not as much value on someone who dropped out in ninth grade but learned how to be an amazing farmer." I'll return to this question in the discussion because I would argue that our ability to recognize our family's epistemologies as valuable in the academy may have relational implications for ourselves, our families, and our experiences in college.

\section{Being “in-between” School and Home: Borderlands (Anzaldúa, 1987/2012)}

While the overarching theme of the findings suggests the work that students do is focused on maintaining connection to home and family, it is worth mentioning a small 
but significant theme that emerged in discussions: the experiences of students who found themselves moving away from home cultures who also remained isolated in school cultures. In the literature review, I wrote about students' experiences of both resisting the goal of integration and becoming border crossers, who sometimes held roles as "heroes" or "outsiders" in their communities of origin after going to college. However, what happens to those who cross the "border" from home cultures to school and find that not only do they remain "outsiders" at school, but will return to home and be received as “outsiders" there as well? Arturo, a working class non-traditional Latino student completing his BSW, described this experience most vividly.

And I heard you say a word, a word "separate" too. Does it feel like there's a separation between those spaces or those cultures now that did not used to exist? Arturo: Right. So I said really, the real, real struggle for a person like me...it's something I don't, I feel like I said, in the middle. Who--where do I belong now? Yeah.

Arturo: 'Cause my people...is my people.

So it's not just separate from, it's not just separate from family and community and home, or community. In a way, is it separate from school too? Is that in the middle?

Arturo: Well, it's, it's, I don't know, Juli. Juli [his conversational partner] is, is...but for me, in my race, so I'm already in--separate. I'm already “other." So...that's why probably I feel more there. I don't know...cause I'm not accepted 
in this... another community, you know? And now I'm not accepted in my...people. In my own group. So it's like, then, what are you gonna do?

Earlier in the conversation, Arturo had spoken about how he felt "okay in school now." Here he was highlighting a subtlety, though: even though he is okay in school, he was also aware of his separation from others in school, that individual-based and institutionalized racism would ensure that he would always be an "other." I found his question "where do I belong now?" to be compelling, and one educators in schools of social work must consider if we are to create schools that embrace social justice. Arturo spoke about being in between school and the professional community and his family and community relationships throughout the conversation and noted how the changes in him that are the result of consciousness raising did not go unnoticed by his family and community members. Arturo described moving away from his brothers and community because he "sound[ed] different" and was "not the same." As a result, not only have his relationships with his brothers and friends changed, but he was left feeling alone:

Arturo: They still...work, they work. I'm a working-class citizen; they're all working class. So they, our relationship change is another challenge.

Yeah.

Arturo: And you know, I resent that. Cause now...I feel like in the middle.

At another point in our conversation, I asked Juli if she felt separate from school and home too, and Arturo interjected, "Well, I used the word 'separate,' but you know, probably I can use 'segregate,' or 'excluded.'” Here is another instance where I felt the subjective and contextual nature of power relationships (Collins, 2000). Juli's voice was 
lost when Arturo spoke first and in place of her invited response. But his response also proffered experiences of oppression. And the relationships between privilege and oppression were somewhat reciprocal again: neither Juli nor I, both white women, interrupted.

Here Arturo's words were heavy: "segregate," “excluded," and "I resent that." He located himself as a working class citizen and noted that his brothers are working class as well. Here it's implied that completing a Bachelor's degree pulls someone out of or away from the working class world, and I think there is some truth to that. Social mobility is often assumed to be part of the college experience. However, Arturo hinted at the losses in this process. He felt less sure of his place as a working class citizen and was now "in the middle." As he'd moved through his studies, Arturo said "I'm kind of lonely." Educators need to engage with Arturo's question: "Where do I belong now?" At the least those in higher education need to be attentive to the potential losses students may experience in tandem with the gains they may make as they earn a degree.

Although it is not as clearly described, Arturo's conversation partner Juli also existed in between multiple worlds. Juli carefully shifted her language in school to demonstrate the appropriate "social roles" in that setting and would shift her language at home to show her family she was still interested in being a part of them and didn't want to appear that she thought she was "too good" for them. But while Arturo felt "okay" in school and spoke about finding comfort in culturally specific services offered at PSU (to be described below), Juli had not found a space like this in the school: 
I don't really feel community or like, home here. 'Cause I feel like I still associate this with like, I don't — I'm just trying to get by. I'm just trying to figure it out enough to...get my degree. And so, yeah I guess I just don't ...I don't...I haven't been connected with anything that I have felt like has been...relatable enough that I could feel like, “Ah, this feels safe.” And I don't feel that in the school of social work...in my experience.

Juli shared this in response to Arturo's description of finding comfort in being with others in the Diversity and Multicultural Student Services (discussed below). Rather than connect with other students, Juli felt that she needed to try to get by on her own in a space that didn't feel like home. Juli seemed between worlds as well, although she may not have felt it as strongly as Arturo. I'll return to this notion in the discussion, as Juli's struggles throughout this conversation to articulate the particulars of social class difference echoed Stuber's (2011) findings about the experiences of white working class first-generation students, who tended to see first-generation student issues as largely being issues faced by students of color. However, in raising Juli's possible sense of being in-between, I don't want to diminish Arturo's. In the discussion of implications of professional socialization I'll return to this, because Arturo's sense of being an "other" at school and in the professional community has profound implications for his success as a social worker.

Before leaving the discussion of students who are in-between, it also seems wise to return to Lauren's description of moving between the "lower class town" she originated from and the "upper class town" where she was a checker. Remember that 
Lauren was attentive to self-presentation in both settings. In the upper class town Lauren was careful to note how long she'd been in her current position and to highlight her status as a manager; she did these things to avoid being ignored or dismissed by customers. But in her home community she risked dismissal as well, both for going to school ("Oh, you're in college? [clicks tongue] "Good for you.") and for pursuing a career in social work. As I considered the distance Lauren traveled between the "upper class town" and the "lower class town" and the work she did in each setting to be read in ways that allowed her to remain in these different worlds, I wondered, where does Lauren get to be herself? It may be that Lauren felt "in-between" as well, although like Juli she didn't articulate this. I'll return to Lauren's self-presentation in the discussion, because it's related to one of the most common themes that emerged in students' conversations: class mobility. Here and in other places, I'm struck by the potential dangers in our cultural narrative of "moving up" in class, which is often associated with earning a college degree. How does the narrative of "moving up" injure students relationally? And how could those of us in schools of social work construct a narrative that values the family histories of all students? Before turning to the second research question I'll share students' relational experiences in school.

\section{Working to "Integrate": Relational Experiences in School}

Examinations of the experiences of first-generation students often focus heavily on experiences in college: how well do students "integrate" socially and academically in the school setting? I've taken a different approach out of the desire to shift the focus to the work students do to stay connected to family, friends, and community. However, 
students' relational worlds were powerfully shaped by their experiences in college and as several students shared, they may feel even more authentic at school as they complete a degree and experience the "transformation" that London (1996) found was part of schooling for many first-generation students. Here I'll outline students' relational experiences in schooling: the original mystification of the "university," experiences of exclusion and inclusion, and the joy some students found in classroom conversations that inspired critical thought and nurtured shared values.

\section{The mystification of the "university."}

In several conversations, the mystification of college emerged as part of students' early adjustment to higher education. Nancy, a white working class MSW student, described feeling behind as an undergraduate, not knowing "how the system worked or what college would really be like. 'Cause all I had heard about college was from like...TV." For many students, the mystification and intimidation was specific to being in a university. Davis described his transition from a local community college to PSU, "I noticed like in my first term I really had to reinforce myself that 'Oh, you have to, you have to step up your game because you're at a university now." Here I understood the importance attached to being in a university as more intimidating or less familiar than community college. Bob shared a similar reaction to entering a university, "I freaked out when I got accepted here - and I transferred here, and I was like, 'Oh my gosh, I'm at a university! This is crazy! Yeah, it was like, 'What!?' This is really crazy, but like, now I'm scared to lose it." Lauren and Bob described their eventual adjustment to the university: 
Lauren: Mm hmm...yeah, I guess growing up I always thought of university as like, super intimidating, um...cause that's what I saw from like the media, like TV shows and all that. And since I didn't have my parents to tell me their experiences, I didn't know and...so when I came here and I got into the CFS program and the professors are so cool, [sounds elated at this last bit] so real, and they want you to engage with them and ask them questions, so...it was pretty eyeopening, compared to like what I thought it was, the reality.

Lauren's initial fears were overpowering. In another part of the conversation, she described that initially she felt "terrified" at school. Lauren's explanation above pointed to the typical first-generation experience: the lack of a familial model or template for college going. However, Bob's response to Lauren's comment highlighted another potential source of misinformation or lack of information for first-generation students: the ways college going is presented in secondary education:

Bob: Um, I don't know, this is interesting, I guess. I've been having the opposite experience, not completely opposite, but I feel like I had a really terrible two years in high school, just didn't like teachers, but then when I came into college I was like, so inclined to go up to them 'cause it seemed more like the environment I perceived as being --at the colleges I went to--more of a freer space, where I feel I could talk to people. So I feel like that I could work with professors--so I guess I wasn't that scared, just more of like, I was more willing to talk to professors, 'cause high school's so different.

Lauren: I think high school makes—or at least the teachers, they make it seem 
like college is going to be so tough--

Bob: --yeah--

Lauren: --and challenging, and when you get here it's like, “what?” [laughter]

What was all the hype about?

Bob: Yeah that senior year they really make it seem like, you're gonna like-

Lauren: --die? [laughter]

Bob: Yeah, die. [more laughter, voice increases in intensity] "You better be ready, cause this is your future!"

Although Bob "freaked out" over getting into a university and has revealed that he is afraid of losing his place in college, it also seems that his relational interactions with instructors in college were a great improvement over his experiences in high school. Here Lauren and Bob highlighted an important source of potential support for (prospective) first-generation college students: high school. However, it seemed that the messages Lauren and Bob received about college only served to reinforce fears about their ability to persist and succeed academically.

In another conversation with CFS students, an additional fear specific to university attendance was brought up: student debt. Davis, who noted that he felt the need to "step up [his] game" once he was in a university, explained that being at a university was different than community college because "you're taking on debt now." This was the second time student debt was raised in this conversation (the first time being Amber's dad's question about "paying off those loans with your fluffy degree?"). But it wasn't the last time debt came up. In the closing moments of the conversation, Tara mentioned a 
number ("twenty grand") and noted that it was worth it to her to be able to pursue work that was meaningful. I can't help but wonder what pressure existed for students going to college within a larger cultural context in which we acknowledge the rising costs of college, including the growing amounts of student debt (Fry, 2012), but offer few clear solutions to this problem. Here too, first-generation status seems especially relevant, as first-generation students are more likely than their continuing generation peers to be working, more likely to be caring for others, and as these findings suggest, more likely to be doing all of this while receiving mixed messages of support from family and community. The fact that these students are paying a much higher price for college than previous generations has implications not only for their futures but likely has implications for their ability to focus on their work as students now.

Closely related to the issue of student debt is the expectation that college should be completed in four years. Again, this is an expectation that's tied to our cultural notions of what college-going is, and first-generation students who don't have familial experience with college-going may assume that they haven't succeeded if they're unable to complete college in four years, even when they are also fulfilling multiple roles, including caregiving and working.

Amber: ... you know, the four-year thing is, I think that was like my biggest thing. I was like, well I have to be done in four years. You don't have to, you know--taking your time, like Davis said, and I think that's the biggest thing I wish I would have known, that it's okay to, like not succeed in that four year time period. 'Cause I was like, dead set on that timeline. [laughs] 
Lizette: So much has passed in two years, I'm like “no, I'm not gonna kid myself'-

Amber: -no, not anymore, but, you know-

Davis: -yeah, it seems like the four-year degree, the title-

Lizette: -the title-

Davis: -it's just kind of like, wow...

Lizette: It's like, it chokes you. It's like, “Ok, I have four years to do this.” If I do a little bit after that, another year--"what does that mean?" and then they start talking about you, “oh, you took that long?” you know what I mean. I don't need this, it's like, who cares? I'm getting it, I'm working towards it.

Lizette's words were important to pause and dwell on. If you don't finish in four years, "What does that mean?" She suggested that this could be worrisome for others observing student progress, "Oh, you took that long?" The questions from family and friends struck me as one of the invisible burdens that first-generation students may carry: those close to them who don't have college-going experience may subscribe to the four-year expectation for a Bachelor's degree. For families without college experience, the traditional notion of completing a degree in four years may be the only blueprint they have for an expected timeline. If students don't finish in four years, this may be read as "failure" to family members who are unfamiliar with the potential slow-downs in degree attainment. In addition, doubts and concerns of close ones may compound any doubts that first-generation students have about their own abilities.

\section{Relational experiences at school.}


Some students spoke of initial experiences of exclusion in the SSW, but for most these experiences were not enduring. Unfortunately, Maria's transition into the SSW was a rough one, and she explained some of the points of exclusion she felt as she was transferring in and met with an advisor.

Maria: ...when I transitioned into college it was like, in a really, you know, just...so, there was a lot of stuff going on and then when I transitioned into college, you know, to PSU, that—'cause I was at [local community college] and then I transitioned to PSU and I went and seeked [sic] advice from one of the advisors in the School of Social Work-

$-y e a h-$

Maria: Or, you know, a person that's in charge of student retention and that wasn't a good experience. And I left, you know, I was almost in tears in her office.

I'm sorry to hear that.

Maria: And for her to just give me a piece of paper, like "Here are the clubs and organizations that you can get into" was terrible. So, and after that, I did not go to any of those organizations or people that she told me about on that paper, so I never got involved in school. And that would have been like, awesome, just to build bigger sense of community in school.

Yeah.

Maria; Just because, like, it's hard, you know, you don't know what you're doing yourself. I didn't know what grad school was. I didn't know the difference 
between like, I knew the name of—but everybody said like, "grad school," right? And I didn't know that was not until actually after your bachelor's and it's like, “Oh, that's what I'm doing here.” And so I didn't know what I was doing. And so to not have any support in the School of Social Work made it even harder.

Notice the layers here: we don't know what was happening for Maria when she came to PSU, but we know that she needed to feel included and didn't feel included by being given a list of clubs. She also spoke to a general lack of knowledge about the higher education process in general which several students have brought up: what is grad school? What is an undergraduate degree? Maria's comment suggested that higher education professionals have to become more explicit in explaining these things to students. I suspect that many of us in higher education, myself included, may assume that students know these things if they aren't asking questions. Part of this response may be based on the assumption that families will help students navigate the process of college going. However, despite her initial feelings of exclusion, Maria noted that other firstgeneration students should "just keep pushing" and "choose somebody else and keep going" if they initially feel unwelcome. Similar to students' descriptions of working to maintain connections to family, Maria's advice to other first-generation students emphasized that students must work (“just keep pushing”) to become relevant. Like family members who remain unchanged, the possibility of the institution adapting to accommodate students is not mentioned.

Maria spoke about a space that she eventually felt affinity and connections with other students, a culturally specific Latina organization on campus. As mentioned above, 
another BSW student, Arturo, spoke at length about one of the few spaces he felt connections to other people, in the culturally specific student services center. Arturo's face brightened visibly during the conversation when he spoke of the one place in the school where he felt relevant and understood. Here there was a computer laboratory, bikes, and services that are culturally specific, like a graduation celebration:

Arturo: ...The Multicultural Student Services... so they told me we have thiscommencement. Give us our diploma. And we will wear this stuff. [Juli chuckles] Pictures and...we'll have the group.

Yeah.

Arturo: And the whole time from the beginning that I started coming to PSU, when I applied for that, it's been like, I come there and I feel different there than other parts of the...

Yeah, yeah. I definitely do--I saw your face change when you were talking about the-

Arturo: It's a sense of that belong-now I am the same level as these other people.

Yeah.

Arturo: Sometimes I feel bigger than some of them [smiles, all laugh]

In contrast, after this exchange Juli described her experiences of never finding a community or safe space in the SSW. Juli's experience of "figuring it out on my own" was reminiscent of Stuber's (2011) writing about white working class first-generation students and the risks they face when white privilege is conflated with social class 
privilege (and their needs may be overlooked). However, here I have to guard against any insinuation that students of color somehow have an advantage, because I don't want to overlook the privileges attendant in whiteness. I'm also aware of how my experiences as a white person with a working class background influence my "read" of Juli's struggle to articulate social class differences. It's frustrating to have class privileges be assumed, because it may leave white students wondering why they can't perform "social roles," as Juli described them, which are actually a product of an upbringing with certain class values.

Continuing with the theme of race and the potential for exclusion, it's important to note the sometimes enduring feelings of exclusion voiced by students tied to racial and cultural isolation in a primarily white institution. As mentioned above, Arturo described being "other" in the school because of his race. I sensed that Arturo read this experience in the school as being a continuation of experiences of being "other" throughout life, and indeed he spoke of his framework for social work practice as one of ongoing "struggle." Another BSW student, a Mexican-American woman named Veronica shared her experiences in the earliest days of coming to PSU:

Veronica: --in our cohort I think that we do have one of the, from what I've heard, that we have the biggest Latino BSW cohort, but then again, it also just depends on the classes you take. You won't see like, Latinos in every single class, in every single BSW class that's offered...so for me it was a big distance of culture and I was like "Oh my gosh!" Like at first, even at the community college where I went to--even in [another large Northwestern city]--it was like "Oh my gosh, am I the 
only one? I don't think I'm supposed to be the only one" From the cultural perspective I would say that...

Can you speak a little bit more about that too, like how that might...how parts of your identity might shape or might make your experiences different as a firstgeneration student or how that piece, um, maybe can you talk a little bit more about that piece of, "I'm looking for other people who look like me"... I don't know if I asked that question right.

Veronica: [laughs] Yeah. I hear what you're asking, um. [whispers] Can you say it again please?

Sure, sure. Can you talk a little bit about what that experience is like, saying, looking for other folks who are Latino? You said, sometimes they're not in my classes. Can you talk a little bit about that and what that means for your experiences?

Veronica: To me, well to me it, to find people in the university or even in the community college that like, you know, look like me or that come from like the same culture as me, um...I guess that would build, I think that would influence my education experience as well, because it would feel like more...right, or like more open, as a person to be more open, more open to, I don't know, to anything that that person brings to the table. I don't know, other ideas and stuff like that too. I don't know, just to me, personally, it will be “Oh, okay, that person is like me. Oh my gosh.”

Yeah.

Veronica: I don't know. To me, that's what it, that's what it means to me, that's 
RELATIONAL WORLDS OF FIRST-GEN STUDENTS

what it - but you know, coming to this program, as well we learned about the word "different" and what that means and what it looks like, so...I realize, coming towards the end of this program makes me realize, okay, I'm not always going to be this person that stands out, or like, is a Latina or first-generation college student. We're all different, so I don't think that's an issue to me anymore.

Here Veronica concluded that it's not an issue for her anymore, and her ideas reflect general teachings on diversity that celebrate difference, but often at the expense of examining power differences and privilege and oppression. But it's important for educators to note some of the ways being in a primarily white institution impacted Veronica's experience. Veronica noted that even though there were a fairly high number of Latino/as in her cohort, she still had frequent experiences of being "the only one" in classes. She described asking, “....am I the only one? I don't think I'm supposed to be the only one." This could certainly erode confidence, as Veronica noted that having more Latino/as in classes would make her feel more open to their ideas and experiences, less unsure, and in short would make her feel more "right."

Veronica's conversation partner, Jayne, a lower-middle class white nontraditional student was confident throughout the conversation and noted that in contrast to Veronica's reticence to be seen as an "information person," she was "flaunting it about." But Jayne also offered a glimpse of her own vulnerability in discussing her initial experiences of exclusion as an older student: 
Jayne: I remember thinking in the beginning, I was like "All these young people, they use such big words."...There was, some people already knew the lingo...And it could have just been me and my insecurities, which I don't have a lot of those, so I had a little. I remember on the first day of school when I was walking up to the building, I took my little video, you know, down there [gestures towards plaza, visible from windows in conference room in school] and talked about how I was feeling about being hereVeronica: ---ooohhh! [sympathetic]. Jayne: So that was interesting. And it's...I remember I came, like everyone back home was like "How'd it go?" and I was like, "I don't know, I feel like I'm the only one there, like I don't know anybody," you know, it feels very um...lonely, that was the term I used.

\section{Hmmm.}

Jayne: But you know, that didn't last very long, but. [looks at Veronica, who laughs].

Jayne also told a story of listening to one of the "young people" in class and learning the "lingo." When another student used the term "problematic," Jayne shared her internal reaction, "Wow, that was such a gentle way of saying 'this is bullshit.", "Especially in the first term, it felt like a little bit of posturing for placement," Jayne said, and it was both different from community college and reinforced Jayne's expectations that she would need to learn the language and nuances of language that are part of social work education, knowledge that younger students seemed to already possess. Here there's 
another link to the importance of which types of language are appropriate, mentioned earlier by Juli. Juli described cursing to show her family she still wanted to be part of them, but Jayne's translation of "problematic" highlighted the ways students are socialized out of language that might feel more familiar and into professional forms of speaking.

Jayne and Veronica both felt that they overcame experiences of exclusion and feeling "lonely" or like "the only one," and endorsed individual solutions to these feelings. Both women resolved experiences of exclusion basically by reaching across differences and forming relationships. Jayne approached her early experiences of feeling lonely and unsure by learning the language and building relationships. "We create that distance in our own mind," Jayne said. Veronica spoke about using self-talk:

Veronica: So, for me, how are we, how I came to now is kind of like Jayne, I told myself things like self, like sending myself things like I-messages, like..."I think," "I believe in you," "Keep on going," Stuff like that. And I, you know...I don't know, kind of like Jayne. I just sort of learned to self-navigate through that. Yeah.

Veronica: Just by, uh...starting the program... and like, still talking to my classmates because I knew they were going to be my classmates for the next two years. So, I just...talked to my classmates, asked them for questions if I had any questions. Just, yeah...everything just, it just-Jayne: --I think the cohort thing helpedVeronica: --came natural to me. Yeah. 
Similar to Juli and several other students, Veronica and Jayne both saw their eventual adjustment to school, what some might call social integration, as resting solely on them. However, Veronica noted that her advisor in particular was especially helpful because He always had open hours, come to my office whenever you need to talk, we can schedule a day if you want to talk. He was really open to anything, even if it was a personal problem. He was always there to provide that sense of like, belonging and "you're part of this, you do matter."

As I've noted in the literature review, there is often an unspoken assumption in writing about first-generation students that they should acclimate to the values and practices of higher education. This expectation overlooks the long-term nature of the investment involved in developing cultural capital and the payoff that Bourdieu (1986) argued was the chief factor influencing different educational outcomes among individuals by social class. Veronica's description of her advisor felt like an exception to me; in this case the institution was bending to meet Veronica's needs. It's important to note that Veronica felt comfortable to come and talk to her advisor and speak freely, even about "personal" issues; being the only Latino/a in so many classes is nothing if not personal. From this conversation, we have no idea if she shared her experiences of racial and cultural isolation, but we do learn that her advisor made a space that was welcoming.

Here too I'm struck by the role of conversations in the relationships that both Veronica and Jayne have found in school. As Jayne said, "I could go to anybody. If I had-if my group, my buddies weren't there [laughs], whatever you want to call them, anybody would... we've shared and know how to talk and listen to each other. [voice 
softens here] We would be there for each other, no matter what." This sense of closeness to other students was echoed in an interview with Dave, a white working class MSW student, who described the experience of being in a graduate program as being one of "raw emotion" and said, "We're being challenged in so many ways and being able to share that with another person who has that experience is very important...I don't have to explain or go into detail with other MSW students, they just get it."

\section{In the classroom.}

Other students pointed to classroom conversations as an important site of connection in the SSW. The graduate students in this conversation didn't necessarily feel closely connected to other students, faculty, or staff, but experienced connections in conversations. Both Michelle and Nancy hinted here that one reason why they're pursuing graduate degrees is because they missed the conversations and learning that were part of their undergraduate degrees:

Michelle: I just remember—so, when I graduated with my BSW I took like a year off, a year and a half, and I just remember like, really missing whatever it was that was at the school. And I've never really been-like I, I'm kind of shy, so making relationships with people at the school, like didn't really happen. And so it wasn't that, and it wasn't like-I didn't really connect with any of the teachers. So I just remember I graduated, like I really missed that space and that environment, and I think it's just like, critical thinking and social analyzing and all of that. Like there's a space for you to do that and like, your viewpoint is valid, whether or not 
people agree or disagree. Yeah there's just something about having that space where you know those conversations are gonna be had.

Yeah. Thank you. [quietly]

Nancy: I relate to that, because I haven't really built strong relationships with very many people here, and not with any of the professors. I actually really hate just having to come talk to the professors [laughs]. Cause I also am pretty shy. Like I don't really talk that much in class...so it feels interesting, cause I did misscause I took off three years between undergrad and grad. And I did miss school a lot. But I wasn't really thinking about the people either. There's just something about—what you're saying about the conversations and kind of having that space to do the analyzing — that really resonates with me. I hadn't really processed it fully yet, but...

...it's so interesting to me, I didn't know how you all were going to answer that question, and it's interesting that conversations like that, that space to have those, is so prominent. It makes me think about, like, reading groups that I've organized with other students and sometimes with other professors here and we'll read something and we'll just sit and talk and think, and um, and I was thinking "could you have this outside of - could I do this with family outside of, you know, school? " And I've found that I can 't. Like, I've found that with my family I just can't, but it's like, there's something that's just so rich about it, like sitting down in a room and throwing around ideas, you know? It's just like—it's very fun. [laughs]. 
Michelle: That's sort of what I was wondering, was like, especially because, we don't feel like we can have those conversations with family, like they shut down or they get made, like that was why it was so important and um—what's it called? And almost like, energizing and inspi---rational, in a way? Like it just filled you up, like you felt vigorous - invigorating?

Michelle's explanation here of why these conversations felt so good extended my assertion that I hadn't been able to have the same kinds of conversations with family that I had in some classrooms, and it caught me by surprise. The conversations firstgeneration students can have in school may be extra rich and important precisely because they're the conversations that are often difficult to have with family. As students have described above, discussions about privilege and oppression and power and identity are often off-limits with family and become markers of separation, tension, or conflict that limit what students feel they can share with family. As they were approaching graduation, Clara asked Michelle and Nancy if they were worried about losing the community, which again existed primarily in classroom conversations. Clara was worried about not having other social workers around to "bounce ideas off of" and Michelle and Nancy both joked about how they'd felt after completing their bachelor's degrees:

Michelle: Yeah, I'm, I'm getting a little nervous about it too. Especially because after I got my BSW, I remember it hitting me, like "I don't know how to not be a student." Like that becomes such a crucial part of my identity. Like you [to Clara] said, we've been in school since we were like, five! [laughs]. Um, and so, I feel like, the pressure, like "Oh, you have your degree now, you're an adult-“" 
Clara: -yeah, you're set to go.

Michelle: Yeah!

Nancy: [laughing] It's a year away, but that sounds really reason-that sounds fair! [laughs, others laugh]. Cause I remember when I did take my three years off, I didn't want to-I took three years off, but then I got, I was like, "I need to be learning something." So then I scraped-I did like a payment plan at [local community college] and I was like-[pauses because everyone is laughing] I took some classes, cause I was going crazy. And I joined like five different book clubs because I couldn't not do it. [laughing] and so...it feels like that was a long time ago cause it was like- - two years ago I guess now. But that happened.

It's difficult to see in this text what unfolded in the conversation: when Nancy confided that she'd scraped together money to take classes at the local community colleges and joined "like five different book clubs," everyone in the room was laughing, realizing they weren't the only ones who loved "social analyzing" and "critical thinking" that was part of classroom conversations. In fact, this revelation seemed like a new one to Nancy, who hadn't thought about why she'd done these things in the years between her undergraduate degree and entering the MSW program. Michelle and Nancy's love of classroom conversations, which they both saw as a privilege, spoke to the important role educators may play in creating a space for students to share ideas that become increasingly central to their identities.

Before leaving the discussion of relational experiences in school, and particularly experiences of connection or disconnection in classroom experiences, one more story 
bears sharing. In this story, Jaclyn, a Native American and white working poor student, described her internal reactions to the "privilege exercise." In this activity specific examples of experiences of privilege or oppression are read aloud and students take a step forward if they have experienced a privilege (or not experienced an oppression).

Conversely, students who have experienced an oppression (or who don't have a particular privilege) step backwards:

Jaclyn:...we had this experiment where everyone who had had some type of experience, some type of privilege would step forward, and if they didn't experience that privilege they stepped back. And I found that it was me and my best friend who came to this program with me-from the same town. Me and her were the only ones in the back of the room with all of the students of color and just that the experience of knowing that, everyone up there makes me feel so uncomfortable now, because you don't know what I've went through, like you don't know my struggles and so it made it more challenging for me to want to build relationships with them because I didn't want to - I didn't think I could relate to them.

And there's literally a physical distance when you do that exercise. People are stepping forward, people are stepping back.

Jaclyn: And you're either on this end or on this end - there's really no... in the middle, so um. And I think, the more I've been in the program, the easier it's been for me to build relationships, but it's still hard for me to connect. And so I've found that it's better for me to build relationships with people that have some kind 
of similar experience because...it...it's harder, I guess, for me, and this is just how I've felt, is just that, um....becoming best friends who's maybe their family's paying for their college or they are very supported by their family, it's really hard to...for them to understand you, and it just...it's hard. I'm not saying it's not possible, it's just...it's hard and I think that for first-generation students there is going to be times when you doubt yourself and have the struggles and it's easier to talk about those things with someone who's kind of went through the same stuff...

Jaclyn's response highlighted a potential unintended consequence of our discussions of oppression and privilege: students' fears of not belonging in school may be reinforced. Educators are already fraught with questions about teaching about privilege and oppression and power and identity, but Jaclyn's story is important to keep in mind: how do we engage in conversations about the very real differences between us and how those differences are related to power without reinforcing classist and racist notions about who belongs in school?

In the next section I'll shift to considering what these conversations about firstgeneration students' relational worlds mean for professional socialization. Students entering the helping professions, and social work students in particular, are called to focus more directly on their development as professionals than students completing a liberal arts degree. Entering the helping professions may be especially meaningful for first-generation students who, as Cole (2008) noted, tend to come from social groups that might be more likely to need supports provided by social work. 


\section{Implications for Professional Socialization}

As highlighted in the literature review, Barretti (2004) urged social work scholars to undertake qualitative explorations of the process of professional socialization for social work students, noting the largely quantitative and psychologically-oriented nature of existing literature. This call resonated with me after reviewing the literature on social work pedagogy in search of studies of the experiences of students of color and students who had experienced poverty and finding the literature lacking. As Barretti (2004) noted, becoming a social worker involves attention to one's own identity and the embrace of values that are shared with social workers as a group. These findings suggest that paying attention to the experiences of first-generation students is important: some firstgeneration social work students noted that they were becoming something that was

feared, dismissed, or had otherwise negative connotations in their family. However, even CFS students, who are entering a broader range of helping professions including youth work, teaching, and other professional roles in addition to social work, had to navigate sometimes complex conversations with family about their professional paths.

\section{Being supported and giving support to others}

Similar to my approach in answering the first question, I'll begin with messages of family support about becoming members of the helping professions. In conversations with students, there were clear patterns of excitement and pride about the work they were learning to do (or already doing, in some cases). Students spoke about carrying on traditions of work that tied them to family history or doing work where they could advocate for others. Sometimes this advocacy was linked to their own experiences when 
advocates were sorely needed. Students also spoke about newfound pride in their work, but this new sense of pride was sometimes laced with the internalized class biases about which types of work are more or less worthy of prestige and honor.

Arturo, a working class Latino BSW student who spoke about feeling in-between the worlds of school and home also spoke about how his education was something his mother had always wanted for him. As a listener I was moved hearing him speak about the confines of gendered and cultural norms that his mother worked within, and how he persevered in school by reminding himself of her sacrifices:

Arturo: I have a mother that even though she...I think is...she went to the second, went to the--maybe six years primary school, and then three years secondary school and then high school-I think she went to the six-six-seventh grade...But she has always this thinking that she want us to, to be something. To all my brothers. And she has this...living in a time, where in my culture where the men is the provider and the woman has to be in the house. So she...has problems with my father, I remember, because she want to do work for another family, like you know, washing clothes, or cooking or whatever, cleaning houses, and like had problems with my father-but she always, because the money wasn't enough, and she want us to go to school: "You go to school," you know?...and I was on border of quitting many times. But then I think of my mother..."I cannot quit again," you know? And then all the money that she spend, like I, even though I-she don't want to see it any—-so I just like, of course I'm gonna do it.

Yeah. That's encouraging, it's encouraging to think "Well, this is what I'm doing, 
what my mother always said. To go to school."

Arturo: She worked all her life just to give us education.

As a listener and reader it was tempting for me to collapse Arturo's experiences into the dominating theme of exclusion that I wrote about above. Both during our conversation and in the successive re-readings of this conversation I was struck by the clarity of his narrative of being in-between worlds: "excluded," "segregated," and "separated." As a person of color and a "working class citizen," he received messages that he did not belong in school. At best, he felt tolerated as an "Other." But this story is also a beautiful description of the potential for connection to family memory or family legacy as a student. These students may be pursuing school largely on their own, but they may also be "carrying" family with them into the classroom.

Jayne and Veronica were two BSW students who both felt that they were carrying on a sort of family tradition by earning degrees in social work. Above I've shared Jayne's story about her older brother, whom she exposed at a recent family event as being so disconnected from her experiences that he was surprised to learn she was in school, but also close enough to her to not be surprised by her choice of major: social work. After telling this story, Jayne noted that her brother's approval had always been important to her, but that he was actually the odd one out in her family, because their parents were "born social workers." Thus, Jayne felt clear about her professional goals and saw it as a continuation of the work her parents had done:

Jayne:...both my parents were social workers. Born social workers. Eighth grade...my dad had to leave school in the tenth grade because it was the 
RELATIONAL WORLDS OF FIRST-GEN STUDENTS

Depression. He needed to go home and... he was very angry about that, a bright man. And my mother, she got to graduate. She was very happy about that. She didn't get to go to college, though...I mean, there was no question... that their kids weren't going to get to go to college if they wanted to. So...they were social workers, they did foster care, they did...I don't know, my dad was just, always helping people, like people in need. Really, they just sort of...found him. And so when I was a little girl, he was a very shy person, but he went to nursing homes in our little town and, and asked, um, to visit people who didn't get visitors... That was the heart of my father. And then he did that, even though it was uncomfortable for him. And they drug us, and we would sing at the nursing homes [laughs] and let people touch us, and so... you know, I, yeah... So this is a continuation. Like, getting your BSWJayne: --for that, yeah. But I just told my brother's story cause it was a little more interesting. [laughs]

Jayne's story resonated deeply with me: by finishing her bachelor's degree and becoming a social worker she will do work in an official capacity that aligns with her family's values of caring for those in need. Jayne's description of her father's anger at having to leave school so early also struck a chord with me, calling to mind my grandfather's sense of loss and bitterness at being let go from his employment because he didn't have a college degree, or even a high school diploma. As first-generation students we cannot undo the harm caused by the lack of educational access for our ancestors. But students 
may draw great joy from pursuing education our elders would have appreciated: Jayne referred to her experience at college as the "best four-year vacation I ever took."

Jayne's conversation partner Veronica felt that by becoming a social worker she would be able to continue advocacy that had been part of her practices for many years, first as a high school student speaking up for a younger sibling with a disability:

Veronica:...in high school, like many personal and family experiences happened such as like also informed, like, my experiences and how I see the world? For example, I think I was a senior or a junior when, like — cause I have a brother, a younger brother-I have two younger siblings. But my younger brother, I think his experience, like with me at least, informed my view to become a social worker, because he was born with a cleft palate... When you're a kid, I think that you get, like, bullied, you know, regardless? And I think he would get more bullied, you know, in school. Like, I felt more sympathetic and empathetic for him, that like, it's like "Oh my gosh," I was super sympathetic and empathetic for him, but like, other of my classmates as well because I saw other classmates of mine getting bullied and...I mean I also got bullied, too myself in high school, but I to me, I think I was like the advocate for these other people as well, I was like [voice gets quiet] "You know, stop bullying this person!" and "blah, blah, blah." Like, 'You don't know his circumstances! - what's behind - like, we don't know what his life looks like, beyond this school setting." So I would just like, stand up for these people. And um, I think that informed my - to become a social worker as well. 
In addition to her experiences of advocating for others and herself in the face of bullying, Veronica felt like she'd developed extra sensitivity due to her brother's experiences.

Veronica seemed to be developing the voice of an advocate, and her voice fell here as she conjured the words of an advocate, "Stop bullying this person!" Her "blah, blah, blah" suggested that there are words that advocates would use that she is either still developing or assumed that all of us around the table knew, by virtue of being in the SSW. Veronica also mentioned service activities in high school with the food bank and Planned Parenthood, and felt that this work for and on behalf of others made social work a natural choice.

Maria was another BSW student who spoke about the positive potential of her work as an advocate, and imagined the work she could do for her broader community. She spoke about seeing mothers of color being denied educational services for their children because they weren't sure how to advocate for them:

I want an education that can, where I can help others and be more involved...I want everybody to just kind of like, learn...how to advocate for yourself if you're in a hospital, if you're not getting-you know in school I see that all the time in elementaries [sic], moms [of color] don't get the same priority as, you know, white families do. And it's like, "Why?"...It's not that I want them to, like, go get an education, like at a college or university, but at least, like to educate yourself on at least, like your rights, so that you can better advocate for your family as well. 
In fact, Maria began the conversation by talking about how her degree was meaningful for her family and community as a potential stepping-stone or resource for other people to ask questions about college and imagine themselves in college, "I want my community to feel empowered and advocate for themselves." Maria's had witnessed racism in unequal access to education and was eager to address power imbalances. Maria imagined her educational experiences serving as a resource to inform other community members both in self-advocacy and to inform others about the path into higher education. If others saw Maria go to college, she reasoned, they could ask her about how they might do the same. However, it's important to note that Maria drew limits on her role as a social worker in her family, an idea I'll return to soon.

\section{Elitist biases... and the valuing of different types of work.}

But students' excitement about shifting into helping professions sometimes highlighted the potential internalization of elitist biases about which types of work are more prestigious or worthy of honor than others. Bob, a working class Latino CFS student, noted that for the first time in his life as a worker he was proud to tell others what he did for work:

Bob: It's, it's really interesting...I remember, [abruptly] sorry! [others laugh] Really quick [others laugh]_it's really crazy, cause I remember I used to work at WalMart, in the back room, for like two years of my life, and I worked at PetSmart and did lots of retail stuff... and then I came to college and I got the opportunity to like_- “Oh, I work with children, and I do like education” and stuff --yeah-- 
Bob: Totally different, and it's almost like—it just feels so interesting, cause like, people ignored me at WalMart, it was like "Oh, what do you do there?" it was like, "Stocker, I work in the backroom and unload trucks," and now it's like, "Oh! You're doing education" and all this other stuff-it's really fascinating and interesting to see the difference. Like, I'm actually wanting to say my career and say what I'm doing, instead of like, I just—you know...work. It's like interesting to see that difference. Like I don't know if it's, like, good or bad, but it makes me feel good...now, cause I'm like, “Oh, I have a, you know, a job," or like a nice career or I'm going towards that end...it's just really different and I don't know how I'm trying to do that, how people respond in a positive way.

I was struck by Bob's words here: "I'm actually wanting to say my career." Bob's view of himself, like the rest of us, was constructed in part by others' views of him. I read Bob's descriptions of his experiences working in other retail positions as experiences of invisibility. As Bob said, "people ignored me," but now "people respond in a positive way.” As much as I was happy to hear Bob's pride in his new career path, I was also saddened by the potential here for Bob to have internalized classist biases about the types of work that are worthy of notice and positive attention.

After Bob shared his newfound sense of pride in the work he was currently doing, other students followed up with their experiences of being judged by others in the careers they're moving into. Here I'm returning to Lauren's words about careful selfpresentation. I've also included Amy's description of where she anticipated landing in terms of class standing, which was relatively similar to her family's middle class status: 
RELATIONAL WORLDS OF FIRST-GEN STUDENTS

Lauren: Yeah, because of the reactions I've gotten from people, whenever people do ask me what I do, I'm like “Oh, I'm a front-end manager for Hagen's, which I've been at for nine years." Like, I feel that I have to tell them that I've been there for a long time to like, bump me up. Or there still gonna be like, "Oh, that's cool—anyway." [laughs quietly]

Yeah... [quietly]

Amy: I guess, my family's, I mean my dad had a, he was like a mediator in a prison, so had like a — it was a good job, worked his way up from a, you know, and but still, you know, we were probably middle, middle-class, basically. And I wanna be a teacher, so I don't really see myself [pauses, laughs] elevating myself above the level of my family that I grew up in. You know, so...but that's fine. You know, I'm not, like, go-getter, looking for the money and [laughs] all the glory and everything. So, yeah.

As I wrote earlier, Lauren recognized the classism in her clientele's responses to her, "Oh that's cool—well, anyway." She made sure to provide enough context for her employment to "bump [her] up," in their eyes. Lauren's scrutiny of how she was read differently as someone going into social work between the "upper class town" and "lower class town" was important here too. She noted that people in the upper class community she worked in responded with approval when she spoke about going into social work, "Upper class people think we're taking care of lower class people," she offered. But as she's noted above, people in her "lower class" town dismissed her as a student and felt she was threatening as a social worker. 
Amy's words took a slightly different turn, but also spoke to class status. In this case she hinted at its intergenerational nature: even though she would be the first to complete a Bachelor's degree, she would probably land in the same class as her parents, based on her father's occupational status. But here notice the invocation of class mobility as a move towards something better or higher: she wouldn't be "elevating" herself above her family, and wasn't worried about income and "all the glory." I would argue that family's educational and employment paths are powerful influences in directing students' educational and employment paths. As Davis noted, having your "generations of people" who have done something before you can be helpful in confirming your educational or employment choices.

\section{Creating a New "Future Route": Family History, Gender, and Cultural Expectations}

Sometimes students felt they were departing from family history or gender and/or cultural norms for their educational path and employment. Becoming a student seemed to involve an identity shift for some CFS students, who had felt like "workers" for a long time but had to shed this identity to focus on being "students." Tara, who had been working for ten years in jobs that she didn't really enjoy, spoke about making the shift from worker to student: "It's been something I've wanted for so long and I finally just committed to student loans and decided to go into it and since I've gone back to school ...I've been working a lot less so it's been a big shift in my life." I wrote earlier about Tara's comparisons of her life and her peers', who had been given ample financial support to attend college. For Tara, shifting from worker to student was significant 
because she'd been on her own for ten years. Amber also spoke about shifting from worker and student to just being a student, because the strain of both roles was so taxing on her physical health that she was hospitalized:

I shifted, three years ago, from working full time to quitting my job and going to school full time because of health issues...work, school, work...my health just couldn't take it anymore. Financially, I wish I could do both [voice softens]. But I just...too many hospital visits, deteriorating health...

Like Tara, part of the economic calculations Amber made when cutting her hours at work to go to school full time involved taking out student loans, a topic that came up throughout the conversation. Hearing both women describe the significance of the shift from worker to student, particularly for Amber, whose health seemed to force her out of performing both roles at once, echoed Choy's (2001) findings that first-generation students are more likely than other students to identify as workers who study, rather than students who work. This identification seems at odds with demands from higher education, where students are typically framed as students first, rather than students who are also workers, parents, partners, children, siblings, caregivers, friends and community members, among other things. Identifying as workers first also is at odds with efforts to centralize integration in school as the primary goal of first-generation students.

Gender and culture may have also reinforced first-generation students' tendency to focus on roles outside of their work as a student. Lizette, a working class Latina CFS student, described the ways that family expectations related to gender and culture were deployed against her in family questions about her work as a student. Lizette's 
professional development was no doubt influenced by competing demands between the many roles she plays: mother, student, worker, and partner. Lizette described the work of these roles as "consuming" and struggled with guilt about her choice to grow, as she described it, through her education. Again, sexism meant that women carried heavier relational burdens; women were much more likely to receive messages discouraging them from schooling and professional development. Nancy relayed her family members' puzzled questions about her decision to pursue a graduate degree, "Why do you want that? Aren't you just gonna get married?" Maria, a working class Latina finishing her BSW degree spoke about dreams of getting a PhD, but anticipated her family's questions and confusion, especially their reactions at spending more time at school: "You're never gonna finish school." Throughout our conversation Maria spoke with passion about the things she was learning and her potential for advocacy in her community. But when Maria imagined her family's responses to her continuing her education, the limitations placed on her by gendered expectations emerged:

but, um, I wanna-like I was telling you, I like research and I wanna eventually get my PhD, but it's like, they're gonna be like, "You're never gonna finish school," and they don't get it, it's like about more — about learning and about, for me, then it's just about getting a degree... So I think that's — that's hard. You know? Cause I think they just think like, “Oh, she's going to school and she’s learning like all these things, like she now doesn't want to cook and clean-

Here Maria's words resonated deeply with me: she dreamt of getting a $\mathrm{PhD}$, and feared not the intellectual challenge or work involved, but the thought of telling family she 
wanted to devote more time to school. Parts of her family's reactions to her were also related to the intelligibility of her career path. Earlier Maria had spoken about the jobs her family felt comfortable with: business and nursing. Her choice to become a social worker didn't seem as clear to family members, and the amount of time she'd already invested in her schooling would surely cause resistance should she decide to get an advanced degree. Maria also noted here the potential for deep rifts in relationships because she was rejecting what seemed to be deeply held cultural beliefs about gender and work: "Oh, she's going to school and she's learning like all these things, like now she doesn't want to cook and clean." However we feel about each person's right to determine their own destiny, we shouldn't overlook the potential relational losses embedded in Maria's choices. Perhaps most importantly, Maria’s own development was potentially limited here by the sum of her fears about moving too far away from family.

\section{Family Voices and Becoming a Social Worker}

Social work students were more likely than CFS students to have family members express negative responses about their professional goals. Sometimes family members' questions were quizzical and stemmed from a lack of familiarity with social work. At other times responses were dismissive, though, or even fearful. Dave, a white working class MSW student was asked the same question by his family that many students struggled with answering, "What would you do as a social worker - what kind of jobs would that bring?" For many students it seemed like family members were struggling to see what their professional jobs might look like, because the work was unfamiliar. Family also equated increasing levels of education with higher incomes. Clara, a lower-middle 
class white MSW student described her family's observation of her advanced degree and the frequent questioning, "You're going to get a good job, right?" Another MSW student, Dave, joked, "They almost feel as if, after I'm done here, I'm going to be independently wealthy."

In other instances family members dismissed social work as a profession because of assumptions about earning a lower income. Lainey, a middle to working class AsianAmerican immigrant, repeated a phrase her father used often (much like Maria's family's “Oh Maria! That's your social work stuff!”) several times throughout our conversation, "Anybody can do social work." Here there was the clear suggestion that the work she was doing wasn't valuable or desirable, or that learning is only useful in terms of economic payoff. Lainey interpreted her father's words through the lens of their experiences as immigrants, where economic resources were critical to survival. Her cultural background also added another layer to his apparent dismissal. Lainey explained that as an East Asian person and a Chinese person in particular, education was highly regarded and also associated with earning higher incomes. Lainey's interpretation of her father's words suggested that his dismissal might be rooted in protection and concern that she avoid economic struggle in the future.

Some family members may have reacted negatively to students' choices to pursue a career in social work because of their own experiences with social workers. Nancy, a white working class MSW student said:

... those family voices do feel meaningful, especially around the word "social work." "Cause a lot of my family members have had social workers in their lives, 
RELATIONAL WORLDS OF FIRST-GEN STUDENTS

and when I said I was getting my master's in social work, there was a very negative response [laughter]. And so I think it does shape, like maybe even, those voices kind of have, like, shaped what kind of social work I'm going into, um because when I first was looking into social work I was really interested in child welfare, but [laughs]...given the response I got from family, I was kind of like, “well, I don't know if that's really the best fit for me." And it turns out I fell in love with something else [laughter], so...it did kind of deter me.

One of Nancy's conversation partners responded to this by insisting that she didn't listen to family because she knew they had strong opinions and didn't want to be discouraged by them, but agreed that "you have to defend your social work status" with family. A white lower-middle class BSW student, Juli, described the support and reservations that her family members had regarding her career choice:

But I think family voices is just like always, um...fairly negative, and it depends on the family, cause I want to say maybe it's half and half, because some are very affirming and very supportive, "That's awesome!" you know, and "You always loved people and what a great work to get into." But then there's like other family that is more like, dysfunctional, and so I think that they're afraid... of me... being in social work, so I think like maybe they don't... have like the best parenting techniques or, um, utilize already like a lot of social-worky resources and so they kind of like assume that I'm gonna be more like critical and judgmental of...them-or, um like look at them in a different perspective, not necessarily as 
like, like somehow I'm gonna lose the family identity and become a social worker...to them.

Notice Juli's words here - there was an element of self-silencing in the back and forth between the descriptions of family support: "fairly negative," but "I want to say maybe it's half and half." It seemed that some of Juli's family members saw her choice to be a social worker as aligned with her personality ("You've always loved helping people..."). But Juli's words about her family coming to possibly fear her were important to attend as well. How many fears are heavier than that of being judged unfit to parent and losing one's child(ren)? How will students fare in their relationships with family if they become that "threat" to their family members? Here Juli was careful, suggesting that family may see her as being more "critical" and "judgmental" of them. Other students were more blatant about their family's or community members' fears about their choice to become social workers "all we hear in the news is... social workers taking your kids away" said Maria, a working class Latina BSW student. Lauren, a white working class CFS student said "lower class people think, 'Oh, you're gonna take my kids away.' That's immediately what they think!"

Another part of Juli's words above highlighted the potential of not simply moving away from family but moving out of the family by becoming a social worker. Juli was concerned that family members might think: “... somehow I'm gonna lose the family identity and become a social worker...to them." Juli’s fears here, ironically enough, mirror the goal of academic integration. Social work students face a real pressure to assume the identity of a social worker Maria, insisted that with her family, "when I go 
home, I try not to be this social work person...my social work role ends at the door." But the push to identify with the values of the larger profession (Wiles, 2013) makes this difficult for students.

Juli's comment also highlighted an important consideration for first-generation students: she may become a social worker "to them [family]." Here she described that not only might her family members fear judgment from her, but they also might see her as a potential resource with a degree in social work:

but now that you have a social work degree I'm gonna ask you like, "I need money for this!" [laughter] or "How do I get this?" and "What do I have to do for that?" just assuming that you have all the answers and the resources.

Juli's words have multiple potential meanings: not only will her identity as a social worker become central for family, but she may also be called on to serve them. Michelle, a white working class MSW student, noted that "I've also had family come to me for help...we have a family member that's going through addiction right now, and they're like, 'Oh! You should do counseling for him!' And I was like, 'That's weird. That's not how it works." First-generation students may be especially prone to be asked to assist family members using their skills and knowledge as helping professionals. As Cole (2008) noted, first-generation students are much more likely to be members of groups that tend to be clients of social work. The fact that first-generation students may be more likely to be continually called to help family members is important to consider. While ethical guidelines limit the possibilities for this type of work on the part of students, family needs are a powerful motivator. How can educators help students prepare to 
explain their own limitations in helping to family? This may be another site of potential “borderlands" (Anzaldúa, 1987/2012) for first-generation students: students may deeply identify with client needs among family members while feeling separated as well.

Simultaneously, students may also identify with the role of helper, but not be able to fully claim it as members of a group that needs help.

As noted above, family members' critiques of students' career choices were more clearly negative for BSW and MSW students, who were becoming social workers, than for CFS students, who were entering a range of roles as helping professionals. However, in this conversation with three CFS students, each student struggled to explain their career path to family because of family experiences that had inspired their choices:

Davis: It's kind of like why... [pauses on this word and draws it out slowly] because I think for a lot of people in a Child and Family Studies program, they're there because of their individual circumstance, or what they've experienced, so it might, you know, be an uncomfortable conversation when the person who might be motivating that, or the group of people, you know, they think "Why are you doing this?" So I think that those conversations are hard to navigate, so...

So are you saying because, maybe because - like maybe our family experiences, or things we experience with our family, make us think, "Oh, I want to go do this and help people," or "I want to go help other people figure this out"... So you're saying that maybe that, that is what makes it difficult. 
Davis: [slowly] Yeah...yeah, but it's not so much, it's not like anger. Just, I think, "why are you doing this?" maybe just some confusion, with the person who's asking. They still encourage, saying "it's great that you're doing that," but it's a mystery, kind of...

Tara: That kind of resonates with me a little bit. Because I kind of, I kind of want to go into child welfare or the foster care system, in a big part because of like, like my dad was a foster kid. So it's always, that's kind of like, resonated with me, like made me care about that and like, domestic violence stuff. But I don't think I've ever told him that that's what I want to do. Because I don't want to make him uncomfortable...

Amber: I think that really resonates with me as well, because like, you know, I always just say I wanna work with kids and at-risk families but I never really say why and I think it's because of like, uh, I don't know, I guess it was just like being a child and having all these health and learning issues, they just weren't really discussed. It was like, oh, you push it off the side, it'll get better. We talked about it, like the band-aid effect and it just like [smiling] so I'm very vague, like “Oh, I felt like I didn't get the assistance I needed to be a successful student.”...I mean, my parents tried. It's not like they didn't, but they used the resources and knowledge that they had. They came from a rural community; it's not their fault. It's the rural community aspect, and the lack of resources that come with being in a rural community. It's just what happens, and I think that's why I've taken such a...niche to like the rural community aspect instead of the urban, because I'm like, I understand the struggle that comes with that...'Cause I think it's kind of 
hard on my mom especially, because growing up she didn't really have, like, the money to get my health in order as quickly as possible. Just different things, you know - they worked full time, you know, they couldn't spend the time to get off work, to take me places that they wished they could have, and that kind of thing...

In this exchange it seemed students risked hurting family by sharing their motivations for going into CFS. As Davis hinted in the beginning of this exchange, family experiences might "motivate" students to go into the helping professions, and explaining their motivations fully might sadden or anger family members who saw students' need to address problems differently in the future as a failing on their part. Tara noted that she was concerned about upsetting her family by sharing that it was her father's traumatic experiences that propelled her to pursue a career in child welfare. Amber was most direct about her own unmet needs as a child with a disability: she felt like there were "bandaid[s]" applied as solutions, and didn't get the assistance she needed. Notice that she was careful to protect family feelings here, saying "I didn't get the assistance I needed to be a successful student," and highlighting the limitations her parents faced by working full time and living in a rural community with fewer resources available. The potential for hurting family members is another factor that may make it difficult for students to be honest and authentic with family about their educational path and career choice, and another potential for distance in relationships. Here too students read their potential growing class privilege as they get closer to degree completion - they may have better solutions to problems their families faced. But they're also sensitively reading power and hiding feelings about how things could have been better. 
Davis initiated this exchange by noting the power of "why" questions from family. Often this was because students were doing work that was unfamiliar to family or not perceived as "real" work. Davis joked that it would be nice to be doing something that his "generations of people" had done because then he might think, "This is what I'm supposed to do." However, it's important to provide some context for his words. In the conversation he emphasized the word "supposed" and feigned a confident voice, suggesting that this was someone else's voice and experience. As someone pursuing an unfamiliar career he felt less sure than family members who chose careers that were understood by family.

In some cases family dismissed the work that students did as not "real" work. Nancy, a white working class MSW student described trying to commiserate with family after a day at work and their response, "Well, you sat down all day!" To her family, a desk job wasn't really work. Nancy also recognized the differences in physical labor exerted in work, laughing about the time she complained to her grandfather about "writing a paper all day" and he responded with, "Yeah...I just chopped up dead animals for ten hours." The question of what "real" work looks like is another potential site of distancing between students and family and speaks to the powerful ways class backgrounds might drive members to police the boundaries of how "work" is defined.

Throughout my analysis I noticed different versions of this line of questioning about their educational choices and career path students experienced. By virtue of the fact that they were doing something that was new to family members, many students had to explain their decisions, sharing their learning and knowledge (which was often met with 
resistance or discomfort), translate the work that they wanted to do to stay relevant to family, and sometimes served as an ambassador or guide for other family members who might follow them to college. As I wrote in the beginning of the findings, family, and friends to a lesser degree, were generally supportive, but quick to share reservations and concerns with students. In listening to students and hearing their words I've come to see these "why are you doing this" and "should you be doing this" cautions from family as a sort of weight that first-generation students must carry on top of all the work they are already doing, pursuing school largely on their own. Not only did these students have to navigate systems that were complex and relatively foreign on their own, but they also carried the invisible weight of family's concerns with them. Family's concerns are often valid, but they may become an additional weight on students and heighten students' own worries about their ability to compete a degree.

\section{Field Experiences: Race, Gender, and Interrupting Oppressions}

Finally, I'd like to explore some students' experiences in field placements, which are important to consider as we think about professional socialization. Field placement and internships offer an important space for learning, as students have the chance to apply research and theory in practice. However, students in field placements hold positions with delicate tensions between being seen as powerful (knowledgeable) and having less power as a "learner." Two students spoke of experiencing racism in field placements, and one student spoke about being dismissed due to her perceived youth and inexperience. In general, students struggled to respond to oppressions in field placements. 
As Arturo, a working class Latino BSW student noted, field experience is powerful for learning, “...the real learning happens when you are in your organizations.” However, Arturo noted that "as a minority, I'm not invited right here [in field experiences]":

...you get your degree, and uh, you say, "sell” yourself, you know, education is for everybody, and call—and it's okay, we came to school, we did the same classes, you and I, and other people. But then you go to the...organizations to do your practice. Well, people at the organizations they have a lot of power, and they can choose to teach you whatever they want to teach you, they give you access, to whatever they want to give you access to...that's where they separate, where the exclusion is...I'm not...included to these groups. You know?...Sometimes when I'm in groups, I feel that pushing back - and then when I finish and how when I sell myself and I'm-where anyway?

Here Arturo was comparing himself to his conversation partner, a white young woman ("we did the same classes, you and I, and other people") and noting that even though they'd taken the same classes, their educational outcomes would differ if he was not fully included in field experiences. Arturo saw inclusion in field as being central to his success: "the fact that I'm a...close to graduation doesn't mean that my situation gonna change, you know?"

While Arturo experienced exclusion and "pushing back" as a person of color, the mechanics of exclusion he experienced based on racism were not entirely clear. In 
contrast, Lainey described the ways she saw stereotypes informed by racism and sexism informing the ways others treated her in field:

Lainey: ...the sense of being minimized also shows up as an Asian American woman, particularly. In my internships, it's over and over again, um, even out of a group of - there are, for example, four interns. I was one of the four, and um, when other people ask questions it was perceived as "Oh, you know, you asked a good question.” But when I ask a question, it was very different. It—-there's some power thing, always some power thing in it, that I'm almost always viewed as lacking in self-confidence, that...I don't know, whatever reason, um told, um...yeah, I don't know...there's just always a sense of, uh...yeah, that I'm looked at as lacking in confidence for whatever crazy reason. And I think there's something, there's something to do with the stereotype of East Asian woman [each of these last three words said slowly, deliberately], period. So as a firstgeneration student coming into these situations, inevitably I just couldn't help but direct that at myself, like is there something wrong with me? How will—how do people show up as being full of confidence? Like, how, how do I, um, you know, how am I supposed to act, am I totally off base? So there's a lot of questioning my own self, my own abilities, just all of these... doubts. So if I wasn't lacking in confidence before-

--right!

Lainey: --I'm certainly lacking in confidence now [laughs]. 
It's like being pre-read, like being read through a race - like a race and gender lens.

Lainey: Right. So that shows up for me, um, and so maybe on some level it relates to feeling like I'm faking....um, I don't really know how else to say that. Maybe there's something wrong with me, I'm not supposed to be here - it kind of reinforces that narrative that... "Oh—you're, you're here! Well. You're not doing these things, you're asking too many questions. Where are these questions coming from?" I remember that was one of the questions that came up for me when I asked about, "Oh, should I file um, is there paperwork for this student who ran into this challenge?" and the reply was "Where are all these questions coming from?"...When other people would ask procedural questions--that seems like a reasonable question to meLainey:--and it's--but they're not received the same way. So I feel, like I'm doubting myself and it compounds the tension that's pre-existing, of being a firstgeneration student and um, being kind of lonely in my household, in my family, of doing all these things that my parents don't really understand, and be told that, "Oh, anybody can do this." So there are all of these things kind of together, in a big bowl... of I don't know what. [laughs] Yeah... Lainey: [quietly, jokingly] Of stress! [laughs] I don't know.

Here it was important to pay attention to the domino effect of the supervisors' racism, which may be so subtle as to be unnoticed by others. Initially Lainey was struggling to be 
confident and receiving messages about the ways that Asian American women are supposed to "be minimized." Lainey was affected by these messages as she wondered what she was supposed to do to be seen as confident. This underlying feeling of needing to be more self-confident was reinforced by the experiences of being in a space where she felt excluded or that maybe the space was not prepared for her "Oh-you're here! Well. You're not doing these things..." This point in the retelling was interesting because Lainey noted that asking too many questions was cause for reproach from her supervisor. It seems to me that the questioning is a different problem than the projected lack of selfconfidence. Notice here too the nod to imposter syndrome: "Maybe there is something wrong with me, maybe I'm not supposed to be here." Certainly it could be easy to feel like an imposter when your mere presence seems to evoke surprise. (Here too I can't help but think of Veronica's imagining how her experiences would be different in classes where she wasn't so often "the only one": she would trust those students more and be open to what they had to say, and she would feel like she was in the right place). Finally I want to point out in this exchange the power of other people's reactions, which Lainey was struggling to articulate. Lainey's doubt as a result of this treatment compounded her feelings of being alone as a first-generation student, including feeling "lonely" in her own home. On top of this, her father's dismissal of the difficulty of this work ("anybody can do this") added to her worries that maybe she wasn't capable enough.

Finally, I want to end with a more subtle and widespread experience to consider: how are students trained out of the language of home cultures and into language that might leave them ill-equipped to interrupt oppressions in practice? Earlier I wrote about 
Jayne, the confident lower-middle class white BSW student who had returned to school to complete a degree. In contrast to her conversational partner Veronica, who was concerned about being seen as an "information person," Jayne noted that she "flaunted" her learning and spoke about it to anyone who would listen. Initially concerned about being an older student and being lonely, Jayne found deep connections with other BSW students in speaking about social justice and "the indignities of the world." But Jayne felt less confident about finding those shared values in field. Jayne quipped about her internal responses to workers in the field, "You're kidding me! You just said that out loud?" I was surprised that even outspoken Jayne, who didn't "mince" wasn't sure how to respond to oppressions in practice. In hearing students' experiences of oppressions in field, which were not a primary focus of this work and emerged spontaneously in several conversations, I want to consider how we can equip students to interrupt oppressions in field and presumably in the work they will do after they graduate. Here Jayne's internal "You're kidding me! You just said that out loud?" that was never externalized may also be linked to the process of learning a professional language. Remember that Jayne focused on learning the "lingo" and softening her language (hearing another student say "problematic" and reflecting "that was such a gentle and diplomatic way to say 'bullshit"'). When Jayne and Juli and other students who tailor their language to fit the perceived norms of higher education that eschew "foul" words and strong language, they may simultaneously lose strategies for interrupting other peoples' offensive and oppressive language.

\section{In Closing: First-Generation Status as a Relational Experience}


As I wrote in the literature review, first-generation status is inherently relational. The framing of students without familial experience of college going as "first-generation" implies that familial experience matters when it comes to getting into, moving through, and finishing college. When students spoke about the significance of their status as firstgeneration students, it was often a deeply personal part of their identity, but it was just as likely to be framed in terms of how their family would experience this new identity. In particular, students spoke about beginning new trends of college going in their families and the significance of being the first to reach higher education. Students also spoke of their place in college as sometimes being a continuation of a role as the family "star" or as the "smart" one or "poster child," but these identifications were complicated, sometimes carrying with them experiences of being misunderstood or guilt at "leaving family behind."

Tara, a white working-class CFS student who spoke above about her experiences of being on her own in pursuing college, emphasized how meaningful her student identity was to her:

I've always felt the need to achieve and finish school even though it's been kind of a struggle and my parents, like, you know, they could, I mean, they care, but it's nothing they are like, "Yes, go do this!" So it's just—it's important to me... at this time in my life it's pretty central to my identity. It's been something I've wanted for so long, and um, I finally just like committed to student loans and decided to go into it ... So it's a meaningful part of my identity right now. 
While Tara felt her parents were initially unclear about her motivations and desires to go to college, they had come to understand why she was pursuing a degree:

since I've gone to PSU I feel like my mom's kind of turned around, I feel like she's very proud of me, whereas she used to be like "well, you just work hard, you don’t need a degree." Because, you know, she ended up being, like she managed a restaurant for 15 years. So she ended up like, kind of, finding her own success, and so she really didn't, like, see the value in it, but since I feel like she's really come around to realizing, like, how important it is to me, and that it's what I want, to be able to have a job that's meaningful to me.

Tara's story was one of shifting family ideas about the value of a college degree. But more often when students spoke about their student identity, it was in relation to their family and how this new identity would change their family members' lives, both existing and anticipated family members, such as children they might raise. Sometimes this notion was still forming, as seen in Davis' suggestion that maybe the fact that he was going to college would be significant for the men in his family, "it's just kind of creating this new...generation of...you know, maybe creating a new cycle." Women were especially likely to articulate a change in future family generations. In another conversation with CFS students, Amy and Brandi spoke to the importance of their roles as first-generation students who would carve paths for family to follow when they pursued higher education. Sometimes they spoke about actual family members, such as Brandi's recognition that her daughter would not have to do the same type of work that her mother and aunts and she herself had done, because of higher education. Notice 
below that Brandi cited a family tradition of being "brang [sic] up" as caregivers as females in a family experiencing immigration:

for people that are Caribbean or Jamaican, they often work in the caregiving field. I don't know why that works out that way, but that's what happens. And so my mom brang [sic] me up as a caregiver, and all her sisters, and I would go to work with them when I was younger, and so when I had my daughter, I was a caregiver at the time, and I would bring my daughter to work at the caregiving home and...I was kind of seeing this pattern, like "this sucks!" --like, it's very low-paying. So I started the journey in college. And it's already paid off so much. My daughter will not have to be a caregiver. Not that caregivers are bad! But it's, it's already--I want to break the cycle. As you say, like your kids will probably go to school, follow in your footsteps, so...

Here I was struck by the relational beauty of Brandi's description of being "brang up" in her family as caregivers. As someone who has intimate experiences with personal caregiving, I felt both struck by the potentially profound lessons that come from the experience of caring for elders - ideally caring for others who are nearing the end of life leads those of us who are caring for them to live ours more deeply and richly. But I'm also viscerally aware of how this work is dismissed in our society, both financially, as Brandi mentioned above, and how it taxes the body and mind.

Brandi also spoke about how her brothers, in their late teens and early 20s, were enrolling in college now. In contrast, Amy spoke in terms of possibility about the impact on her future children, "if I have kids then they'll probably end up going to college 
because I did." But she felt very clear about the significance of her student identity for supporting the young people who were in her family, feeling “...proud to be an example for my three nieces and my nephew, and just excited to be a resource for them, in hoping that they decide to go to college someday. I'll be able to answer those questions that my parents weren't able to answer for them." Here too it's important to emphasize that firstgeneration students see themselves as responsible for not only their success but for the potential success of others. This is similar to the responsibility they feel to continue reaching out and maintaining connections to family, efforts that may not be reciprocated by family members.

The lack of a familial model for college going is often assumed to be a deficit for first-generation students and Lainey, an MSW student, shared that view. But Jaclyn, a Native American and white working poor MSW student, disagreed with her conversation partner Lainey's assertion that familial experiences with higher education would have helped her, saying, "I don't know if I would have come as far if I...had someone who went as far as me...this is a chance for me to set that bar in my family and have other people look up to me and do better than me." Jaclyn felt that if other members of her family had gone to college that she might be caught up in comparison, asking herself "am I as good as this person?"

But Jaclyn spoke most vividly about her daughter when she spoke about the significance of her identity and starting a new trend of college-going in the family, which Jaclyn described as "changing generations to come." "I think of it as like we're changing our family...roots. Like I said with my family, it goes far beyond just my immediate 
family, it's a much bigger piece. And I look at it like, as this is me changing generations to come, hopefully!" Jaclyn spoke positively about the opportunity to set an example for her daughter to reach for early in the conversation, but as we continued speaking, she brought up more of the complicating details of her story that are important to consider in our conversations about pedagogy. Jaclyn spoke about the lifelong impacts of poverty, including the domino-effect chain of events that led to where she is today: during high school, a guidance counselor offered her support in completing college applications but could not convince her parents to share their income information, which is required for FAFSA. Jaclyn opted to get married and ultimately the marriage didn't last. Jaclyn is now raising her daughter largely on her own and faces emotional challenges in completing her degree which I fear too often escape the attention of educators and professionals focused on student retention:

And I feel like, here I am struggling to make ends meet and like, at times, I don't know how many times I've felt like, “Gosh, I just can’t do this anymore.” I had to quit my job and move up here to go to school, and so-and that sucks you know, like having to rely on loans, and only loans, even the loans that we get aren't enough and, and just thinking "Gosh - I have to pay this back" and then...you know, getting a scholarship means you get less...loan money, so is that really beneficial for me to accept this scholarship or not, and so -cause that's great, it's great money that I don't have to pay back, but is it going to support me and my daughter? You know, it's just really, it really causes a lot of stress. I mean I definitely have never regretted my daughter, but it does like...there's rallies and 
RELATIONAL WORLDS OF FIRST-GEN STUDENTS

things that I'd love to participate in, but I just can't, you know? I don't have daycare after 5:30, I don't... and you know, there's a part of me that justespecially first term, I needed a job, I needed more money, and so, but where do I fit this in? Like, getting a part time job, it's really just gonna pay for my daycare to be at that job, because-you know, how is it really gonna help me? And she's only two, so it's like, I'm missing so many milestones and I tell myself, "Gosh, if I would have just stayed in my hometown and continued working my job and making money and just waited until I was in my late thirties and she was grown up and out of the house, I wouldn't have to worry about missing any of the stuff with her." I wouldn't have to be financially struggling and um, yeah, that's a challenge and...it's hard.

At this point in the conversation, I felt moved by Jaclyn's sadness and was compelled to highlight the potential positive impacts in Jaclyn's earlier narrative of changing generations to come, offering this:

Those "what ifs" are really heavy, but there's also the piece you were sharing at the beginning of "BUT, I'm carving this path"-those are my words, your words are better, but-I'm also making this path for her. So that's a real, that's a real balance.

Jaclyn: That's the only thing that really holds me on is I know that...in the long term, my daughter will be proud of me. And it sucks now, especially like for Christmas, I know she's too young to know that, I can't afford Christmas presents and stuff, but it doesn't sit well with me. So it's definitely challenging, but I just 
keep telling myself that [crying] it will all be worth it, and once it's all done, it will all be better. So...

I've written about debt already but it bears repeating because those in higher education may not all be cognizant, at least not on a visceral level, of the great burden many students are carrying in terms of debt. In this exchange, Jaclyn doesn't seem comforted even by the idea of changing generations to come. She was vividly aware of the sacrifices she was presently making that impacted her and her ability to feel like she was caring for her daughter the way she would like to. This strain was so great that Jaclyn wondered whether it would have been better to just wait and pursue college when her daughter is older. Jaclyn's story seemed important to think about in terms of the larger structural problem of massive student debt. As a society it seems we are perpetuating inequality by forcing students who cannot afford the steeply rising costs of college to be saddled with debt for years to come.

Jaclyn's story is important to pay attention to for another reason, because like many students, Jaclyn's responses endorsed the notion that getting a college degree was somehow a better path than many of the paths taken by family members in young adulthood which involved work and parenting:

I know that if I would have been like my parents and not graduated high school and lived in a small town and worked at a mill, or worked at some, you know, low-paying job that was really labor-friendly, um...I think that what kind of example would I set for my daughter and that's not really the example I would want to set. 
Jaclyn's pride here at going to school resonated with how she described herself in the first minutes of our conversation, as feeling "successful" and "honored." Jaclyn also noted that she was always considered to be the "family star" so it may be fitting that she's been able to beat the odds and not only complete a bachelor's degree, but also a graduate degree.

But there's also a competing tension in her questions here about the different paths into adulthood one can take and the relative value of those paths. Jaclyn asked what kind of an example she would have set if she had stayed in a small town and "worked at a mill" or other "low-paying job." It's clear that Jaclyn has read the world and absorbed the message that this path is not as worthy as the path to college and a professional career. Jaclyn's words here have me thinking about how first-generation students can resist notions that our parents' and grandparents' work and lives were somehow lesser than or less worthy because of a lack of education. Jaclyn has also clearly considered the possibility of following in her parents' footsteps - in fact, she's noted that it might have been better for her economically, because of the large debt she's had to take on. Jaclyn was being pulled between competing expectations for her choices. On one hand, there's the dominant cultural narrative about college being the best choice in young adulthood (indeed, sometimes we tell the story that it's the only path to take into getting a living wage job, a story which is as dangerous as it is untrue). On the other hand is the wisdom in Jaclyn's father's rejection of college as unnecessary, "Who would you rather have dig your ditch?” Viewing Jaclyn's story holistically reveals how strongly each narrative 
pulled at her, causing her to question whether college was the right choice even as she felt successful and honored.

\section{Moving up? Troubling the narrative of social mobility}

Jaclyn's question about the type of example she would have set for her daughter if she would have stayed in her town and worked at a mill also highlighted the embedded classism in our societal valuing of college and de-valuing of trade work. In her line of questioning, the classism is fairly obvious. However, in listening to these conversations, I became aware of how the dominant narrative of college going as a means of social mobility, while largely true, is also laced with classist and racist implications, especially because this notion of college going as a move "up" was endorsed primarily by students of color. This is a much more subtle point and it may be hard to spot: it's a widely-held belief in dominant U.S. culture that completing a college degree is a good thing. I suspect that my drive to take a close look at this narrative may strike some as unnecessary or even discouraging of college going. But as I listened to students talk about "reaching" higher education and moving above or beyond their families' places in the world, I couldn't help but wonder how this construction of college, which absolutely does provide benefits, might also be harming students' relationships with their families.

As noted above, the view of getting to college being a step up from family was endorsed often by Latino/a students, which makes me ponder not only the classist but also racist implications. Lizette, a Latino working class CFS student, said she felt "great about myself, that I accomplished something my parents couldn't do. For being the status, how they were and how they were based..." Veronica, a Mexican-American BSW 
student, similarly noted that she felt grateful to be in school because "I'm in a setting where my parents couldn't potentially be." Veronica was also the first student whose framing of higher education as something that one "reaches" struck me, "no one in my family has ever reached higher education." It's important for me to note, though, that Veronica doesn't seem to have absorbed negative ideas about her family or their work, and she highlighted that the reason she was in college was due to having different "opportunity and resources" than her parents had. Maria was another Latina BSW student who explicitly framed her move into higher education in terms of the immigration experience. Here the language of "reaching" educational attainment shows up too:

...of course none of my family attended college. My mom stopped at fourth grade and my dad in middle school, so...I mean, they didn't even reach high school and my sisters, some of them, had got their GED, so it means a lot to me that I'm able to come to school and have this education. 'Cause I know, like down, down the road, I want my community to be--feel empowered and like advocate for themselves and I think that if they have somebody within their community that's going to college, then it's like, "Okay, you should go here, or you should go there, maybe you should try this." I don't know, I feel—it means a lot to me, just being able to come over here. I was just thinking about, um, in like graduation, if I'm gonna like say something at my dinner, right? And I'm like, "What would I say," and I was thinking a lot, and it's like, well, my mom worked in the fields forever and so did my grandma and they had to move to a, you know, whole different country. And so it's like, yeah--I'm very proud of that I'm able to come this far. 
And so, just I guess, it's not only my work, they did a lot more to it. Yeah, I'm like the one getting the degree, but you know, who really like suffered, um, through that, like migration and stuff? So yeah, so it's really meaningful to me, to my mom, to my sisters and stuff.

Maria's words spoke to several things: the notion of increasing educational attainment as a move up, the meaning of her education not only for her, but also for her family and community, and finally to the immigration experience. Her words were powerful: "I'm the one getting the degree, but...who really suffered?" While I'm sensitive to the potential damage of students absorbing the messages that their family are somehow less than them because of their lower educational levels, I also felt moved by Maria's recognition that her ability to reach higher education was the result of the sacrifices and struggle of multiple generations of women working hard and that the degree was not hers alone to claim.

I was also compelled by Maria's descriptions of her education as being about something that will benefit her community in addition to her, and will contribute to forward progress. I've wrote elsewhere of Maria's struggles to remain in relationship with family by thinking carefully about which beliefs she could share with family, but she also advised other first-generation students to "challenge their [family] thinking" because if they didn’t, “we're never gonna move forward.” Here Maria is clearly endorsing the notion that higher education, and challenging family beliefs when those contradict learning in higher education, is a means of moving up or moving forward. There is potential damage in students seeing moving "up" and away from family as a 
move towards something “better," or in some cases like Lauren's, being good enough for the "upper middle class" worlds they inhabit. Here Maria's words complicated my own resistance to the moving up or forward narrative: her advocacy in the many communities she identified with as a woman, as part of a family of immigrants, as a Latina, as someone who was working class, and as someone who identified as LGBTQ also struck me as a move forward towards a more inclusive future. I think I was also softened in my resistance to the moving up narrative because Maria was also deeply rooted in the struggle to maintain relationship with her family and community. However, as I've indicated above, it's important to trouble the narrative of higher education as a move up, particularly for students of color who are moving into a primarily white institution.

However, before leaving this conversation, it's important to note cases where the narrative of moving up seemed less benign or perhaps more infused with rejection of class backgrounds. Lauren's description of moving between the "upper class town" she worked in and the "lower class town" she came from highlighted this, as she noted that her dismissal by others in her "lower class" hometown as a student and social worker because perhaps people in that town just "didn't... want to get themselves out of the lower class town." The notion of escaping the town suggested that there was something to escape, and again Lauren suggested status differences when she described the attention she gave to her appearance when she returned to her "lower class" town as an intern with the Department of Human Services to work with families in poverty: Lauren noted that she was trying to "bring it down...to their level." 
I don't think these were messages that Lauren invented on her own, and I wonder how those of us in higher education reinforce these notions of good and bad, or the "good enough" Lauren strives for in her work in the upper class town, and "better" (the presumed status of people in that "upper class" town). Even though Lauren also knew what it felt like to be looked down upon, it was striking to me that she had probably also absorbed messages about people in the lower-class town being "less than." I think examining the narrative of first-generation students "reaching" higher education is important in considering how students internalize oppressions about race and class, especially when discussing helping relationships. I'll return to this conversation in the discussion; there are important relational implications for helping relationships for someone coming from a group that is perceived as being "less than" who then works with members of that same group in a power-up relationship.

Finally, I want to close this section of the findings with students' descriptions of their identity in relationship to family. Students acknowledged the privilege of being a student and sometimes noted that they had always been seen as different or "smart," but these identities were complicated and carried relational consequences. Jaclyn, who carved the path to higher education in her family, said she'd always been the hero and the "family star." In a conversation with three white, working-class MSW students, Michelle spoke of herself as a "poster child" in her family because she had not only graduated from high school and received a bachelor's degree but was weeks away from completing her Master's degree. With this designation came pride, but also shame. Michelle recognized that she was doing something that wasn't just big for her, but for her family as 
well. But she also spoke of concern for how her first-generation "poster child" status impacted her family members who might compare their own academic progress to hers and said, "I experience a lot of guilt sometimes too, and blame...for leaving family behind, quote unquote." Michelle also worried that she would discourage family members who were interested in higher education if she was honest with them about the time investment involved in her degrees:

Part of me is like, even afraid to explain that, though, because especially for the younger people in my family, when I'm like, “Ok, well a bachelor's is four and a master's is two more on top of that," a lot of them are like "No, not for me." Just by the sheer number. So I almost hate when I get asked that, because I'm like 'No, I want you to - like, if you're considering college, do that. Don't get discouraged by the time" [laughs]

Michelle's explanation of the ways her "poster child" status influenced her relational experiences in her family prompted Nancy to respond this way:

I think what you're saying kind of reminds me of that guilt, because I think I've felt that feeling kind of, like really yucky to have gotten this far, and there's a lot of guilt with that, because I've gotten the same thing. Especially from my grandparents, of being like, the special kid. And I feel bad, or I feel guilty, and bad for my younger cousins, and for my brother. Because- regardless of what, whatever they want, is what they should do, but it kind of gets framed in this way of "Oh, Nancy did that because she could. She's really smart." And I hate it when 
RELATIONAL WORLDS OF FIRST-GEN STUDENTS

they call me really smart. I hate it so much, because it kind of discounts the work that goes into it. Like, "Oh, she's just really smart."

Nancy's voice rose here in the recording "I hate it when they call me really smart. I hate it so much..." Nancy, Michelle, and other first-generation students have beat the odds and gained access to higher education. Their families may indeed see them as "poster children" or "smart" or the "family stars," but these labels are not free from drawbacks and complications. Perhaps these complications can be addressed by continuing to name and possibly diminish the distance between the cultures of home and school. Here Brandi, a working class Jamaican-American CFS student, explained the importance of connection to family in shaping her own identity and the ways schooling worked against this:

I mean, honestly, in some ways my quality of life has decreased because of being a student...I don't spend as much time with my family or my friends, sometimes I don't even know who I am anymore because most of my free time is often spent doing homework or doing these other-academic things. And I wonder like, as I get older if-I think my degree will---I'll be really proud of it, because I am so passionate about it, it's just—at the same time, will I have regrets about my youth? It's a long time...you're sacrificing a bit of your life. Because even though it's a portion of yourself...you know, this is your time almost to be a little selfish and like, take care of this really important thing so you can get back to society-I do feel like it's not a short amount of time that's being asked, and...you don't really know what you're missing out on until you reflect back. 
Brandi highlighted tensions common to many college students, regardless of generational status ("It's not a small amount of time that's being demanded" and "You're sacrificing a bit of your life."). Brandi also expressed her passion for her degree and tentative embrace of the student role: "this is your time almost to be a little selfish." But she also highlighted the assumed separation of the cultures of school from not only home cultures but "society" ("take care of this really important thing so you can get back to society"). This perceived isolation of academia seems especially salient for first-generation students, who as the literature indicated, sometimes struggle with integration. The potential for distance created by spending time away from family didn't only impact Brandi's health but also her sense of identity: “sometimes I don't even know who I am anymore." Brandi paused before elaborating, cautiously, "I think, for my perspective, I think academia's a little out of touch." This study is my attempt to narrow that gap. 


\section{CHAPTER 5: DISCUSSION}

Much of the scholarship on first-generation students has focused on their academic and social integration in college (Collier \& Morgan, 2008; London, 1989, 1992; Lowery-Hart \& Pacheco, 2011; Padgett et al., 2012; Pascarella et al., 2004; Stuber, 2011). While family and community are sometimes mentioned, the ability to integrate and become "intelligible" in higher education is the central focus. This focus on integration has been informed at least in part by the dominance of Tinto's $(1975,1993)$ Theory of Student Departure. While Tinto's theory has been widely criticized, the core assumptions that students need to separate from communities of the past and integrate into college are still reflected in approaches to supporting the retention of first-generation students. Here I've broadened the focus to explore the relational experiences of firstgeneration students who are working to integrate or become "intelligible" in school while also working to remain intelligible and connected to home cultures.

These findings also speak to Bourdieu's (1986) notion of embodied cultural capital, another theoretical approach common in studies of first-generation students (Collier \& Morgan, 2008; Padgett et al., 2012; Pascarella et al., 2004; Stuber, 2011). Embodied forms of cultural capital include the attitudes, experiences, and lifestyles that are valued by those in higher education (Bourdieu, 1986). Bourdieu theorized that these values, perceptions, and experiences were usually formed over many years, and that certain types of cultural capital were valued in schools. Interventions that are designed to provide first-generation students with the knowledge and skills to succeed in school are a nod to the cultural capital framework; if first-generation students can gain the knowledge 
that they presumably lack from familial experiences with higher education, they'll be better able to integrate in college and remain.

The experiences of first-generation students in this study highlighted the burden these students face in being (or becoming) outsiders in the multiple worlds they inhabit. In the school, first-generation students were learning to see and articulate systems of oppression and privilege and learning new "social rules," particularly around language and conversations. One of these lessons is typical content in schools of social work, but lessons in "social roles" and language and conversation highlight the presence of the "hidden curriculum" (Giroux, 2001). Educators in schools of social work often witness much of this learning. But first-generation students were also doing a lot of unseen work as well. Part of this involved finding spaces and people that helped students feel comfortable in places which often felt exclusionary, at least at first blush. For students who never found a safe space in the school, this also meant just trying to learn the "rules" and get through school.

Students also did work that went unseen, to remain in connection with home cultures. Students described translating their daily lives to family and friends for whom their experiences were completely unfamiliar. Students also worked to forecast their future professional paths for family members who were uncertain, threatened by their work, or sometimes dismissed it as not "real" work. These findings confirm the need to see first-generation status as inherently relational: while students embraced the individualist notion of student hood, they did so with acute attention to what this new role meant in their families and communities. While there is a growing body of literature that 
touches on the relationships of first-generation students, relational experiences have not been a focus. This is surprising, given that first-generation students are framed in relationship to family experiences of education.

What lessons do these findings offer for educators in schools of social work, practitioners, and researchers? First, it seems important to acknowledge the powerful role of home cultures and their support. Students felt like most family and friends were in support of their choice to complete a degree. However, students were largely on their own in terms of instrumental support. This led to a few different problems for firstgeneration students, the first being significant financial distress and concerns about debt. The second problem leads into the next theme, the potential for growing distance from home cultures. While students "did school" on their own, their daily lives grew increasingly separate from (and less legible to) family, friends, and community members. However, rather than prioritizing social and academic integration, which theory and intervention emphasize, students were almost universally committed to remaining integrated in home cultures and did significant work to stay connected. It's useful for educators and researchers in particular to see this work that first-generation students do to remain integrated in home cultures as well as the work students do to understand, navigate, and thrive in a school of social work.

\section{Family is an important source of support}

Tinto (1993) argued that before they could integrate socially or academically in college, students needed to separate themselves from their communities of the past. He likened the transition into college as a rite of passage. This belief reflects an assumption 
that students want to separate from their home cultures. However, this was not the case for students I spoke with, many of whom were in daily or weekly contact with members of their home cultures, especially family. Students often began conversations by speaking about the support they received from family in pursuing a degree. This support was primarily emotional: families were proud of students and their work, even when going to school meant taking time away from family and doing work that seemed foreign. This finding confirmed studies in which first-generation students spoke to the ways family supported them (Bradbury \& Maher, 2009; Gofen, 2009). Emphasizing the support students draw from family challenges some of the assumptions about first-generation students, the first being that family have a small role to play in supporting students because they lack experience with college. A lack of a familial template for college framed studies of academic differences (largely, deficits) in studies by Padgett and colleagues (2004), Pascarella and colleauges (2012), and Terenzini and colleagues (1996). Academic deficits are also borne out in longitudinal studies with nationally representative samples (Chen, 2005; Choy, 2001). A lack of the appropriate types of cultural capital is often employed as an explanation of these differences: college demands work and knowledge on the part of the student and family. Despite these families' inability to provide much of this support in the way of cultural capital that was valued in higher education, family support was still critical.

A second, and less benign, assumption about first-generation students is that their family members don't value or support higher education. As Gray (2013) pointed out, sometimes these assumptions are even built into interventions supporting students. This 
study highlighted family contributions and will re-shape the narrative of family as having little to offer first-generation students or holding them back from academic success.

These findings also paint a more complicated picture of the nuances of family support, though. Support from family was peppered with questions and caution; exploring the reasons for these queries, challenges, and critiques is important for social work educators. Family members' concerns about the high cost of schooling seem valid. While I didn't directly ask questions about student debt, it arose in multiple conversations as a tension for students. Sometimes this was shared by family, as in Amber's father's words about paying “off her loans with her fluffy degree?” Conversations about student loan debt are increasingly part of public discourse; one in five households in the United States now has student debt (Fry, 2012). Since 2007, student debt has grown at both ends of the income spectrum: the lowest-earning fifth of all households and the highestearning fifth both owe more than they did in 2007. But the repayment burden falls differently on these groups, with the highest-income households paying between 2 to 7 cents per dollar of income towards their student loan debt, while households in the lowest fifth of the income distribution pay $24 \%$ of their income towards student loan debt. It's not surprising that family would have concerns about debt, particularly families which may have experienced financial difficulties (fourteen out of the nineteen students identified as "working class," "low-income," "working poor," or "poor," and four identified as "lower-middle" class. One student did not identify a class background). Because students are the first in their family to invest in a bachelor's degree and pursuing these degrees in a time when mounting student debt is of great concern, it is little wonder 
that families may be apprehensive about the economic investment. Bourdieu's (1986) notion of embodied cultural capital may be especially salient here: attitudes, values, and perceptions will differently shape our opinions about the value (and wisdom) of investing in a college degree.

Another potential source of mixed support from family that warranted attention is the elitism embedded in messages about college as a means of social mobility. On the surface, this narrative is embraced as a meritocratic means of rewarding students who work hard, and social mobility is presumed as a goal worth aspiring to (Giroux, 2009; Haveman \& Smeeding, 2006). Family members don't need college experience to ascertain the benefits of a degree. Our dominant cultural narratives promote college going as a way for students to gain entry into (or keep their place within) the middle class.

Although many family members were unsure about accessing college and navigating the processes involved in moving through college, they knew there were benefits. Again, because these students overwhelmingly identified as being "working class," "lowincome," "working poor," or "poor," social mobility may hold a special value in family messages of support.

However, this narrative of college as a means of social mobility is relatively unexplored in terms of the potentials for relational injuries. Just beneath the surface of our messages celebrating students' accessing higher education is another message with classist undertones (or sometimes overtones). In addition, the differential societal values ascribed to people based on educational attainment and occupational prestige may be at the heart of some family members' tepid responses and mixed messages of support 
students received. As Jane Van Galen (2014) has pointed out, family members of firstgeneration students may indeed show resistance to changes in students when they appear to be embracing aspects of middle class culture associated with higher education. If family members have experienced classism or elitist treatment from those with higher levels of education it stands to reason that they would speak up when they saw people close to them embodying those attitudes, ideas, and values. This was evident in the ways several students downplayed their knowledge, trying not to "brag" or become an “information person," or taking care not to be seen as "uppity.” Dave's father's “Oh, I can see you've been to college" hints at how college changes people and serves as a subtle warning to Dave about the potential straining his relationship. In this view, mixed messages of support may serve as a means of maintaining connections to students. While proud of students, families may also be reminding students of their bonds to family.

These mixed messages of support alerted me to more carefully consider the language of the narrative of college as a means of social mobility. It was clear in these conversations that students have absorbed this message too. Students spoke of "moving up," "reaching," higher education, and "getting out" of a "lower-class town." These statements have become part of our vernacular about college access for students from underrepresented groups; indeed I've caught myself using the term "moving up" in writing this dissertation. But it's important to pause and explore the relational weight of these words: what does it mean to "move up" or to "get out" of the places we come from? What does this say about the people and places we come from and our changing relationships to them? While it was hard for me to view some students' pride at 
"reaching" higher education with a critical eye, I couldn't help but wonder about the internalized classism that Jaclyn felt when she asked what kind of example she would have set for her daughter if she'd "lived in a small town and worked at a mill" or when Bob noted that he was finally proud to tell others what he did for a living. True to our cultural narrative, these students are moving up. But how can we celebrate their achievements without simultaneously de-valuing their past work or the work of their loved ones? Here too Tierney’s (1999) cautions about Tinto's (1993) emphasis on separation and integration are important: how can Jaclyn experience "cultural integration"? Tierney argued for re-envisioning the academy and a greater democratization of schooling with greater inclusion, rather than continuing to place the burden of integration on students.

The conversation about the dangers of relational injury and reinforcing internalized classism may be particularly salient in the helping professions, when students may be helping members of groups they also identify with. I was struck by Lauren's careful balancing act to be accepted in the upper class community she worked in as part of the service industry and accepted in her "lower class" hometown, where she was increasingly seen as a threat based on her internship with the Department of Human Services. Lauren was critical of people in both of these communities: she risked dismissal by wealthier people in the upper class community but knew she could garner their respect by emphasizing the fact that she was a student. In this community her role as a potential social worker was applauded; Lauren suspected that wealthier people saw less wealthy people as being "taken care of" by social workers. She was dismissed in her hometown 
for the very same reasons: being a student was looked down upon, and being a social worker was especially frowned upon. Lauren worked to "bring it down to their level" with her appearance, but this language is worth examining with an eye to the potential messages Lauren has absorbed about the place she's come from. Lauren's assessment of her hometown is plain in her explanations of the rejection she feels in her "lower class town": maybe other people who live there just didn't want to "get out." What does it mean for students who are members of the helping professions to be in positions of power over people who may be like them, who may come from the same "lower class" town, who may come to view them as a threat? What does this mean for students' sense of professional identity? And what does it mean for their relational worlds, to see groups they may identify with constructed in terms of their risks, deficits, and weaknesses? The potential for antagonistic relations between first-generation students and members of their home community is important for social work educators to consider. This is an area that needs further exploration in literature on social work pedagogy, as the scant literature on poverty and student experiences was focused on how students view poverty or why they decide to work with those in poverty, rather than the educational experiences of students who have experiences in poverty.

As noted throughout, support from home cultures tended to be largely emotional in nature. Family offered encouragement and praise; instrumental forms of support such as help applying for school, accessing financial aid, or paying for school were limited among the students in these conversations. In terms of the financial cost of schooling and figuring how to get through college, these students were largely on their own. 
RELATIONAL WORLDS OF FIRST-GEN STUDENTS

\section{"Performing without a net": Students are on their own}

Historically college students have been constructed as autonomous individuals who spend four years investing in their own education and development, although as Renn and Reason (2013) pointed out, this student profile is increasingly one of historical interest. "John," the white, Christian, heterosexual, middle class, able-bodied traditional aged college student described in the introduction who lived and worked part time on campus was treated as an individual who in our collective imagination held sole responsibility for his academic success. However, in reality even "John" was reliant on layers of support in his education, including probable economic support from family and hefty government investment in higher education. As Giroux (2009) has pointed out, higher education has seen deep cuts in state funding at the same time access has increased for members of underrepresented groups (Carnevale \& Strohl, 2010). Students on many college campuses, particularly in public institutions, look less and less like "John": they are more likely to be poor, immigrants, and people of color (Giroux, 2009). These students are entering college at a moment in history when public investment in higher education has decreased and tuition increases have outpaced other living expenses. In addition to lacking the societal investments that got "John" through college, these students are more likely to lack the familial investment as well, at least in terms of money. However, it seems that often those of us in higher education are approaching students today with the implicit assumption that they have the same resources and support as "John." Emphasizing integration asks students to take on someone else's identity, in a context lacking supports for college going. 
These conversations with first-generation students revealed that their lives as students only faintly resembled the traditional model of college going. Their identities as college students were often meaningful, although this role was not always central to their identity. Several students spoke about the value of investing this time in their development as individuals and pursuing work that was meaningful. But the overarching theme of their conversations was that they were not only doing something new in their families but also doing it on their own; like Bob indicated with his cupped hands when he spoke about his anxieties about his school performance, students were performing without a net. Often, they were the only person they could rely on for instrumental support of their education. This has clear economic implications, but it also has deep relational implications as well.

I've already written above about debt but it bears repeating: the share of student debt has grown substantially since 2007 and the share is heaviest among the youngest and poorest (Fry, 2012). Students raised concerns about debt multiple times in these conversations as a substantial part of their anxieties about school. Borrowing money to do something no one in their family had done seemed to present its own challenges for students. In part this seemed to be about fear, as students pondered the wisdom of their choices about loans. As Nancy and Michelle noted, it's “terrifying” for someone who's not wealthy to embark on adulthood with a high amount of student debt. Davis steeled himself for university-level coursework, primarily because of the greater investment it required and the need to "take on loans." Other students weighed the relative costs of taking out loans versus working outside of school. Whether they accepted student loans 
or worked more hours to avoid taking them, considerations about student debt were at the forefront of several students' minds when it came to completing their degrees.

But pursuing school on their own and accruing student debt had relational implications as well. In follow-up member check sessions this spring multiple students noted their fears about revealing how much student debt they had to family members. As I've noted in the findings, most students didn't question the fact that paying for school was their economic responsibility. It was unclear whether students and family had ever had any conversations about the cost of schooling; for many students and family members there seemed to be an implicit agreement that students were on their own. In member checks students who were now nearing graduation, some of them considering furthering their education, spoke in hushed tones about keeping their student loan balances secret from their parents, or keeping the fact that they'd taken any loans to themselves. Collier and Morgan (2008) wrote about the need for first-generation students to understand faculty expectations, but it's also imperative for faculty to be aware of the broader contexts of students' lives as it relates to debt. As I write these words I am aware of how deeply implicated I am in these questions of how much to share our economic investments and burdens with family. My own debt, while relatively modest for a graduate student, would shock and frighten my family members. I don't know that I can carry their concerns about debt as well as my own. I suspect that students may feel the same way. But this secrecy presents another point of potential distance for students from their families. Here too, Gergen's (2009) ideas about remaining intelligible are relevant: assuming large sums of debt may indeed seem unintelligible to family and friends. 
Secrecy about student debt also contributed to students' internalization of the potential for failure and may prevent students and those who support them from connecting "private troubles" to "public issues" (Mills, 1959). This isolation prevents the possibility for collective actions that could potentially restore the types of support that college students used to enjoy, in terms of collective investment in higher education. This type of hopeful speculation may be beyond the current scope of this work. But the relational struggles of hiding student debt and buying into the myth of individual responsibility were salient in these conversations. As mentioned above, students balanced the costs of their schooling with additional work and loans. Some students like Lizette, who didn't speak about loans, struggled under the weight of being a worker, mother, and spouse, in addition to student. Many students opted for some combination of loans and working in addition to school. But their rhetoric reflected some of the language used to describe first-generation students' struggles, particularly with "time management" (Collier \& Morgan, 2008).

In their study exploring the incongruence between faculty expectations and firstgeneration students' understanding of those expectations, Collier and Morgan (2008) noted the need for first-generation students to prioritize and improve "time management." Several first-generation students in these focus groups noted that they "tended to overcommit" (p. 437). Similar ideas emerged in Davis' reflection on his own experiences as a student in contrast to his "generations of people." He recognized that he was creating a new path and departing from the one his (presumably) male family members had followed: graduating high school, having a girlfriend, having children, and, of course, 
working. Doing something different meant that Davis found himself struggling with time management. By focusing on his need to learn to manage time better, Davis located his struggles in his individual experience, and as the students throughout these conversations have done, placed the burden of integration on himself.

It may be that Davis simply needed to learn time management. But what about Lizette, who woke up hours before the sun rose each day and prepared herself and her two sons for a four-hour stint at work before coming to school, and then returned home with her children twelve hours later, to face the daily routines of feeding, cleaning, and bedtime? After bedtime she might find time for schoolwork, but how much energy could she possibly have after such a long day? Will Lizette's problems disappear if she improves her "time management"? This is where discourse about the needs of firstgeneration students often rooted in a cultural capital (Bourdieu, 1986) framework falls flat for me. A faculty member in Collier and Morgan's (2008) focus groups offered this, “We are an urban campus. We're designed to deal with people's life crises, but I think the great gift you give to them [students] is to share we're all limited by 24 hours a day, 7 days a week" (p. 432). On the surface this is true and if accepted at face value then it would seem reasonable to teach and emphasize time management as a tool for student success. But in listening to the conversations, particularly the experiences of Lizette, Brandi, and Jaclyn, who are parents as well as students, it's hard for me to accept the idea that they just needed to adjust their priorities. How many of these students were failing to manage their time? How many of them simply had too many tasks to manage in the amount of time they have? As this faculty member noted, we're all constrained by the 
same limits of time. But the roles we have and responsibilities that are part of those roles can vary widely; the tasks we have to accomplish can look very different in those 24 hours. As I've noted in the literature review, it's well established that first-generation students are more likely to work longer hours, to work off campus, and to have caregiving responsibilities (Bradbury \& Maher, 2009; Orbe, 2004; Pascarella et al., 2004; Terenzini et al., 1996). How can we as educators in schools of social work better consider the contexts of first-generation students' lives while still supporting a rigorous education? Perhaps remembering how much these students were on their own can help educators begin to imagine how to re-shape classrooms and institutions that were designed for the "John" of the past to respond to today's students. Whether educators embrace this invitation to re-conceptualize the discourse of "time management" or not, it's important to note that students absorbed these messages and saw their success and failure as their something that was largely their own as well. This internalization of fault, combined with the potential for distance and self-silencing in conversations, contributes to the "unintelligibility" in home cultures that students are working to address.

Finally, the experience of being on their own in school also carried relational weight for students as it marked the growing potential for distance from home cultures for students. Students' daily lives seemed unfathomable to students and this led to frustration, such as Jaclyn's continued attempts to explain her work to her parents each time she visited. It also led to relational discord, particularly for women, who fielded hints and outright accusations from family about the amount of time they were spending as students and their lack of contributions to family. As I've noted throughout, it is 
expected that first-generation students will work to integrate themselves into the school setting and some degree of difficulty is both documented and expected. But the demands to remain integrated in home cultures are strong as well. Bradbury and Maher (2009) wrote about the "pull of home" and about first-generation students' observations that they were no longer able to connect in the same ways to former friends after spending time at college. But these students not only felt the "pull of home" but also the possibility of moving outside of its gravity entirely. In keeping with the metaphor, these students worked to stay within the orbit of home while also adjusting to school culture. Whether you see them as bridges or astronauts or something else entirely, these students were pulled in opposing directions and worked to maintain solid connections in each. The fact that they see this responsibility to stay grounded in each context as profoundly their own is important for those of us who teach first-generation students to be aware of. Similar to London's (1989) writing, students felt pressured to "break away" and were also lulled by the "pull of home" (Bradbury \& Maher, 2009). But this work expanded the focus to explore not just integration, but the dual nature of students' work to integrate and remain integrated. The limited literature on relational experiences outside of the school may be due to underlying assumptions that students will separate and integrate (Tinto, 1993) and to the too-often apolitical readings of Bourdieu (1986), in which forms of cultural capital that are valued in schools are treated as commodities which can be gained and used to support integration.

\section{Conversations and integration go hand in hand}


In almost every conversation with these students the notion of "there are some conversations I just can't have" with family came up. I came to see the ability to engage in authentic conversations with others as central to students' ability to integrate in school and to stay connected at home. For most students their ability to share their ideas and feelings freely with family and to some degree friends was hampered by growing tensions. Many students found comfort in having conversations about their learning, particularly around oppression and privilege, in the classrooms and with other students. But many students had been disappointed by their attempts to replicate these conversations with others in their home cultures.

Sometimes breakdowns in communication seemed to surprise students, such as Bob's attention to the words his friends said when they talked on the phone ("words that I wouldn’t use”) or Lainey’s cautious push back against her family member's biases. Juli noted the danger in responding to these conversational faux pas, though: “...not laughing, calling out, that immediately puts you back to being, like... 'There she goes again.'...totally excludes you.” The relational weight of Juli's words in this exchange bear repeating, "[I] still want to be a part of this family." Students struggled with decisions about how much to share with family and how to say it. Students often opted to self-silence in conversations with family to maintain their connections.

Students were aware of how education had changed their consciousness, especially about identity, privilege, and oppression. Students also recognized their individual privileges in being able to learn about these ideas. While many students were familiar with experiences of oppression, especially racism, students also shared that being 
in school had given them new language to name and articulate the relationships between aspects of their identity and their relationships to power. As Dave and Arturo noted, they'd distanced from family in learning about these things. Here too first-generation status is salient because students were already marking themselves as different from family by pursuing a college degree. Confronting family with conversations about oppression and privilege in particular presented additional tensions on relationships already strained by the potential for distance.

Questions about how to support the translation of conversations about oppression and privilege are especially important for educators in schools of social work. How can educators prepare students to enter into conversations about oppression and privilege in ways that are easily translatable to settings outside of the college classroom? Most students are unlikely to remain in higher education for their entire careers so it is reasonable to consider how conversations about identity, oppression and privilege can look in all the places students will practice: in group work, private practice, case work, in schools and classrooms, in youth work, in health care settings, in policy advocacy, and the many other places students will enter. Perhaps preparing students to carry these conversations beyond the walls of the college classroom will have the benefit of helping them enter into these conversations with family and friends as well. Exploring these conversations in the classroom with students, both the successes and the struggles, may incorporate aspects of cultural integrity (Tierney, 1999). Tierney noted that part of expanding access to higher education included making space for multiple perspectives and inviting disagreement into conversations. 
The question of conversations is also relevant for community-based practitioners and policy advocates as well as educators. Social work practitioners doing communitybased work know that conversations about identity, oppression, and privilege are not abstract theoretical concepts but are tied to the struggles of many groups working to address poverty and economic injustice, racial injustice, reproductive justice, and to advocate for policies that address the unevenly gendered burden of caregiving in our society. First-generation students know many of these issues intimately and their family members do as well. Maria tried to have these conversations, noting that her family understood racial discrimination in labor practices and white privilege. How can practitioners and educators support future social workers (and potential doctorateholders) like Maria in these conversations? Here too creating space for students to voice their experiences of attempting to transfer or share their learning into home cultures may provide important support.

Closely related to conversations and integration are the work students were doing to integrate (and remain integrated), namely modifying conversation, code-switching, and careful self-presentation. In the school setting we expect that first-generation students will have to do some work to integrate and learn the rules of this new space. But we aren't always honest about the power relations embedded in this process: first-generation students are coming into schools from a position of less power. Questions about the "social rules" of the school and which types of language are appropriate are part of the hidden curriculum (Giroux, 2001). The hidden curriculum includes the implicit lessons in the classroom which transmit messages about which attitudes, behavior, knowledge, and 
experiences are valued. All of these ideas are germane in classrooms in schools of social work, especially regarding questions about what it means to be a professional. Educators can engage in clear conversations with students about professional language, including when (if ever) “cursing” is appropriate? (In my experiences, professors" "rules" about this vary widely!) Language was especially critical for first-generation students. Jayne and Juli both wondered about the guidelines for language and modified their language in attempts to be professional.

Conversations about professionalization can also include exploring what it means to embody professionalism in terms of behavior, dress, and interactions with instructors, clients, and other students. Perhaps most importantly, we can invite students to challenge the elitist underpinnings in professionalism and consider the relational implications and power dynamics in what Delpit (1995) refers to as the "culture of power." Who decides which types of language are appropriate, and in what contexts? Who determines what it means to look and behave like a professional? What types of resources are required to affect this self-presentation, and who has access to them and who doesn't? And to return to Lauren's work to "bring it down to their level" when visiting people in her "lower class" hometown, what are the relational implications of self-presentation for helping relationships? Educators don't have to have all of the answers to these questions, but considering the power dynamics embedded in questions about language, attitudes, and behavior (in short, cultural capital) in the helping professions presents a way to reenvision classrooms that are responsive to the needs of first-generation students.

\section{The work of integration: How ready are schools for first-generation students?}


Pages upon pages have been written about the struggles of first-generation students to integrate. It's less common for these discussions to be framed in terms of the ways classrooms and institutions are ready (or not) for first-generation students. Here I'll highlight possible points for faculty and staff in schools of social work to consider how to better prepare for first-generation students. Some findings require little adjustment, such as awareness of the feelings and questions students may bring. Other findings point to deep-seated problems of racial and cultural isolation and the ways schools perpetuate institutionalized racism. Finally, seeing first-generation students and their potential needs is important for educators to consider.

Several students confessed that there were significant knowledge barriers to getting into college and getting over the feeling of being able to stay, especially at a university. These barriers weren't limited to the typical imagined concerns of firstgeneration students, though: how to enroll in classes, how to find your way around, and who to ask if you need help. Many students confessed that being at a university was so strange as to almost be unthinkable. Remember Bob's shock at learning that he'd been accepted into a university? Nancy and Lauren both said that the only things they knew about college were from depictions in popular media, and Davis prepared himself to "step up his game" when he transferred from a community college. One student admitted to throwing up on her first day of classes out of sheer anxiety. Cultivating an awareness of the anxiety a college campus, and especially a university may present for students who are "new bodies" is an important step for faculty and staff who may be the first people first-generation students meet. 
Students also described bringing a host of general questions about higher education that they had figured out on their own. Many of them were now explaining these things to family as well. But one student described initial experiences of exclusion in the school related to questions about the differences between an Associate's, Bachelor's, or Master's degrees. “Everybody said 'grad school,' right?” Maria said, noting that no one ever explained that was the degree she might complete after her BSW. An interaction that might pass by unnoticed for people well versed in higher education may seem terse to a student who is not only intimidated by being in a university but also by their lack of knowledge about school.

Unfortunately the experiences of racial and cultural isolation that Daniels (2007) found among students of color in social work were echoed in some of the experiences of first-generation students in these conversations. Veronica's description of being the "only one" in too many classes was reminiscent of the same racial and cultural isolation. While Veronica felt that she had adjusted and found friends, her words are important for educators and administrators to consider: "Am I supposed to be the only one?" Veronica's words also provide a glimpse into the added tensions for first-generation students of color in a primarily white institution: she felt less likely to connect with other students, less likely to trust them, and less likely to trust that they would understand her. As she said, she felt less "right" when she was the only one. Arturo's questions were more pointed than Veronica's: "Where do I belong now?" His family, friends, and community members all recognized the changes in his ideas and language. Arturo also saw these changes, and his language was forceful: he had become "impregnate[d] with 
these ideas of social justice" and he emphasized three times in the conversation that he was "not the same." In the findings I've highlighted the relational weight of his experience: "segregated," "separated," and "excluded." Because even though Arturo had learned the language of social work and moved away from family and community, he realized that here he was still an "Other" and still not fully included. As Tierney (1999) noted, the integrationist perspective that flows out of Tinto's (1993) theory is colorblind. From this limited perspective, Arturo and Veronica's experiences may lead to a "failure" to integrate. Similarly, Collier and Morgan (2008) demonstrate first-generation students to master the student role, a laudable goal, but one that would be improved by a critical perspective on experiences of racism among students. Gaining knowledge or skills to perform the student role may not be enough to support students experience "exclusion," "separation," and "segregation" to integrate. For that, an institutional response is required.

Arturo also pointed out the ways exclusion followed him into field placements and the potential impact on his professional career. Field was "where the real learning happen[ed]" but he had the clear sense that he was "not invited" to fully participate. I've highlighted his questions in the findings: while he and Juli were both receiving a BSW, their outcomes would not be the same if he faced racial discrimination in field. Essentially, experiences of racism made Arturo question if he was better off after completing his education. I found myself wondering what the breakdown was for Arturo in field and what was happening to send this message of exclusion. His questions and challenges are important to consider because they interrupt the narrative of first- 
generation students as struggling to integrate and suggest that institutions of higher education bear at least part of the responsibility for being "ready" for first-generation students to feel invited.

Lainey offered a more detailed description of her experiences with racism (and to some degree, sexism) in field, and her interpretation of what was happening spoke directly to questions of schools being "ready" for students from underrepresented groups: “Oh-you're, you're here!” This expression of surprise on the part of others in field was immediately spun as a failing on Lainey's part: "You're not doing these things, you're asking too many questions. Where are these questions coming from?” Lainey's conversation about her experiences in field highlighted the different ways she was perceived and penalized based on racism. She was told she lacked confidence and came to see herself this way, wondering what she needed to do to effect an outward appearance of confidence. In contrast, when she asked questions (an action that may suggest confidence) she was met with resistance. It seemed like Lainey was in a no-win situation in her field placement and that racism in particular had something to do with it. Here too I want to emphasize institutional roles in supporting students in integrating: how can students feel confident in perform well in spaces in which their fears about being a "new body" are confirmed by others' responses that suggest the space isn't ready for them?

Another issue to consider is the ways that first-generation students are framed in terms of race. Jane Van Galen (2014) has written about the ways first-generation students are constructed by those in higher education as the "exotic 'Other"” who (surprisingly) feel out of place in college. Jenny Stuber (2011) noted that even white first-generation 
students tended to see the issues of first-generation students as being tied to race, rather than generational status in school. Stuber noted especially the ways that whiteness might function as a risk: some of the white working class students in her study faded into the background and their needs were unmet. This finding loomed in my thoughts as I listened to the conversation between Arturo and Juli. While Arturo was clearly hurt by the experience of being an "Other" in school, Juli was also struggling on her own to "figure it out," a phrase she used three times in the conversation. When Arturo spoke about the respite he found in the Multicultural Services Center, Juli said that she couldn't name any space in the school where she'd felt safe or "relatable." Juli's invisible struggle to figure it out on her own is reminiscent of all the work first-generation students were doing "on their own," to adapt to all the spaces they occupy. It's important to consider how our popular ideas about first-generation students might make Juli's needs go unseen, for faculty and staff and even for her.

Finally I'd like to think about we shape our classroom conversations to be ready for first-generation students. I've written extensively above about the possibilities for classroom conversations that name the hidden curriculum (Giroux, 2001). I've especially focused on conversations about oppression and privilege, but Jaclyn's story about ending up at the back of the classroom highlights the need for educators to carefully consider how they unpack privilege in classroom work. If they are not careful, conversations about oppression and privilege can re-victimize students who experience oppressions (Arao \& Clemens, 2013). These authors write that oppressed students can often predict the outcome of the game from the beginning and privileged students are surprised (and 
defensive). In Jaclyn's case it is unclear if she was surprised by the outcome of the exercise, but the message was clear: she was different from the people at the front of the room. Whether Jaclyn's goal was integration or not, this experience may have reinforced the difficulty, or impossibility, or ever achieving that goal.

\section{Limitations}

Throughout writing I've felt acutely aware of the limitations of this work: I've asked participants to self-identify in terms of demographic details (and indeed even in terms of generational status), the conversations were limited to one-time focus groups, and with a relatively small number of students. Finally, I've been considering the limitations that are uniquely my own, as someone with complicated relationships to some of the participants, who knew me as an instructor before we sat down in these conversations. I'll address these briefly here.

Following our conversations I asked participants to provide demographic details that were important to them, attached to their selected pseudonyms. I suggested that race, class, gender, sex, and ethnicity might be important, and invited participants to share other information that felt important (and they did, sharing about age, sexual orientation, and religion, among other things). I quickly learned that including sex and gender through students off, even in a school of social work, because these terms are so often used interchangeably. I relied primarily on race, sex, and class when writing about students in the findings because these aspects of identity seemed most salient in many of their conversations. Based on student's broad categorizations of class, I concluded that most students were roughly "working class" or "low income" (the latter was used by one 
student and suggested that his identification within this class might be temporary; most students used the term "working class"). There are certainly limitations in my relying on participants to self-identify, but it seemed like these were things that I trusted participants to know much better about themselves than I could.

The second limitation, collecting data in one-time focus groups, may have limited perspectives from students who were just beginning to think about their experiences as a first-generation student. Ideally, multiple conversations would have allowed us to more deeply explore relationships and the shifting nature of students' negotiations of their relationships to family and others in their home cultures as well as in school. However, I found it somewhat difficult for all the interested students to participate, given the hectic pace of students' lives. While 19 students ended up participating in focus group conversations or in an individual interview, the number of interested students who contacted me was at least double. Focus groups offered an important benefit, though, in prompting students to reflect on the meaningfulness of their experiences and the possibility of shared experiences. In these conversations I witnessed many moments of a student pausing and then offering, "I don't know if anyone else does this..." only to be met by confirmation of shared experiences by their conversation partners. Witnessing the moment of being "not alone" for so many students was an honor, and in most conversations we stayed to talk with each other after the recording was done.

Finally, I'm deeply cognizant of the limitations that I've brought to this work. During transcription and analysis I saw the gaps in my facilitation and the spaces when I felt my voice was too present. As I've mentioned above, my relationship to the 
participants was complicated. I felt acutely aware of my novice in the first focus group and noted during the group and during transcription how my role as "instructor" might have influenced students' words and stories. Fortunately this seemed to be less of an issue in subsequent conversations, both with students that I had taught and with students from social work programs, who were largely unknown to me beforehand. In reading and re-reading transcripts I noted the places where my facilitation faded into the background, such as in Clara, Michelle, and Nancy's conversation about how it felt to be unable to speak with family. I'm still working to achieve what Morgan (personal communication, September 11, 2011) referred to as the ideal fit for a facilitator: doing as little as possible. Part of this struggle was also about my relationship to the topic: students' sharing sparked many of my own struggles in remaining in relationship with family while also creating a space that feels tenable in higher education. As the conversations progressed I worked to speak less, although it also seemed to help to frame my position as someone who was also first-generation. Throughout writing I've been aware of the possibility of framing the findings through my own experiences, but embracing the partiality and incomplete nature of my view (Harding, 1993). In reading and re-reading transcripts I've been struck both by voices that spoke so clearly to my own heartache and struggle as well as students whose reactions caught me off guard. I know that I cannot erase my influence entirely, and I feel fortunate to have pursued work that is so close to my heart.

\section{Implications}

Throughout the discussion I've written of the implications for educators in schools of social work. Here I'd add a few ideals for educators, particularly those in 
schools of social work. Ideally, educators interested in supporting first-generation students would reclaim higher education, to use Giroux's (2013) ideas, as a space for inclusion, and developing a "culture of teaching, learning, and questioning" (Giroux, 2009, p. 14). This invitation to questioning might have smoothed to processes of learning the rules at school for students and made integration easier. Carefully considering the materials used in teaching and learning is important too; as Clara noted, some types of knowledge weren't valued in school. Giroux (2009) suggested turning to popular culture for pedagogical resources and explicitly confronting the question of whose knowledge is valued (and indeed, even considered "knowledge"). In considering the potential for educators to support integration, approaching classroom community as "brave spaces" (Arao \& Clemens, 2013) rather than "safe spaces" may create space for students who feel aware of how different their backgrounds are from other students. The shift is not merely rhetorical, as a "brave" space anticipates conflict as a fundamental part of conversations across difference. Finally, while political advocacy is not always at the forefront of educators' minds, I would join Giroux's (2013) call for educators to work to protect higher education as one of the last spaces for "education as the practice of freedom" and to resist further changes rooted in the embrace of neoliberalism in higher education.

I've also highlighted above the ways these findings might be beneficial for social work practitioners, particularly community-based workers who may encounter firstgeneration students in their work. Sharing ideas about the translation work that firstgeneration students are doing in the multiple spaces they occupy may ultimately lead to the redistribution of these burdens among more members of their home and school 
cultures. This work is also relevant for those in private practice, who may benefit from hearing students' tensions they face in staying connected in their home cultures while pursuing a degree. College completion is framed as an almost universally good thing in our dominant discourse, but here students have been able to convey what they risk losing as well as the work they do to maintain those connections.

Finally, I'd like to offer my modest contribution to further research on the relational worlds of first-generation students. I anticipated that my most important contribution would be to learn how to apply a form of data analysis designed for singleperson interviews to focus group conversations. In repeating this process and mapping Brown and Gilligan's (1992) questions onto subsequent readings and re-readings of transcripts I developed a sense of focusing both on individual speakers and on the meanings constructed in exchanges between speakers. In analyzing the conversations I came to see participants' discussions as braids that diverged and converged at different points. In some moments, such as Clara, Michelle, and Nancy's exploration of being unable to talk with family about the things they cared about, the braid was tightly wound. In other parts, such as Arturo and Juli's conversation, which was marked by entirely different raced and gendered experiences as well as different conversational styles, there was more separation and turn-taking in conversation: their braid was loose, but still bound together, if only in their invitations and rejoinders to each other: "I don't know if Juli feels that too" or "can you relate to that?" The four readings of each transcript allowed me to become deeply familiar with each conversation and I'm eager to write 
about applying this method to focus groups, if only to appease the needs of other novice researchers who are working to de-mystify the process of qualitative analysis.

I anticipate that the finding that students work to maintain connections will be fruitful for future research on first-generation students, and will hopefully expand the focus beyond solely considering integration in school. This work, while largely focused on students' remaining integrated in home cultures, also invites researchers to consider the relational nature of academic and social integration. The focus on integration contains embedded assumptions that students will embrace the attitudes, behaviors, and beliefs valued by higher education. To some degree, students have limited choices in this matter; students must affect these attitudes, behaviors, and beliefs to be "intelligible" in school cultures. This work offers beginning steps towards Baretti's (2004) call for more qualitative explorations of the professional socialization process in social work as well. In students' stories we are confronted again and again with the power of family voices in determining their educational and employment options. These students were pulling away from family and creating new "future routes," but those paths were still directed to some degree by culture, gender, class, and family history. Learning to see these influences in their wholeness and complexity and honoring the work students do to stay integrated in home cultures is an important step in supporting first-generation students in schools of social work. 


\section{References}

Abel, J.R. \& Deitz, R. (2014). Do the benefits of college still outweigh the costs? Current Issues in Economics and Finance, 20(3). Retrieved from: http://www.newyorkfed.org/research/current_issues/ci20-3.pdf

Anzaldúa, G.E. (1987/2012). Borderlands: The new mestiza = La frontera. San Francisco: Aunt Lute Books.

Arao, B. \& Clemens, K. (2013). From safe spaces to brave spaces: A new way to frame dialogue around diversity and social justice. In L.M. Landreman (Ed.) The art of effective facilitation: Reflections from social justice educators, (pp. 135-150). Sterling, VA: Stylus Publishing.

Barretti, M. (2004). What do we know about the professional socialization of our students? Journal of Social Work Education, 40(2), 255-283. Retrieved from: http://web.b.ebscohost.com.proxy.lib.pdx.edu/ehost/pdfviewer/pdfviewer?vid=3\& $\underline{\text { sid=14719efd-6229-46a4-91c1-d9ecc1cda3b0\%40sessionmgr115\&hid=125 }}$

Bean, J.P. \& Eaton, S.B. (2000). A psychological model of college student retention. In J.M. Braxton (Ed.), Reworking the student departure puzzle (pp. 48-61). Nashville, TN: Vanderbilt University Press.

Beard, P.D.B. (2006). The influence of a select federally funded program on firstgeneration college student success. (Unpublished doctoral dissertation). Texas Southern University, Houston, TX. 
Behar, R. (1996). The vulnerable observer: Anthropology that breaks your heart. Boston, MA: Beacon Press.

Berger, J.B. (2000). Optimizing capital, social reproduction, and undergraduate persistence. In J.M. Braxton (Ed.) Reworking the student departure puzzle (pp. 95-124). Nashville, TN: Vanderbilt University Press.

Berkner, L., \& Choy, S. (2008). Descriptive summary of 2003-04 beginning postsecondary students: Three years later. (NCES 2008-174). Retrieved from U.S. Department of Education website: http://nces.ed.gov/pubsearch/pubsinfo.asp?pubid=2008174

Bourdieu, P. (1986). The forms of capital. Trans. Richard Nice in J.G. Richardson (Ed.), Handbook of Theory and Research for the Sociology of Education. Westport, CN: Greenwood Press.

Bowie, S.L. \& Hancock, H. (2000). African Americans and graduate social work education: A study of career choice influences and strategies to reverse enrollment decline. Journal of Social Work Education, 36(3), 429-448. Retrieved from: http://web.a.ebscohost.com.proxy.lib.pdx.edu/ehost/pdfviewer/pdfviewer?vid=3\& $\underline{\text { sid=7ebbefd5-6667-4084-88fb-6ee6befa72b8\%40sessionmgr4004\&hid=4107 }}$

Bradbury, B.L., \& Maher, P.C. (2009). The Integration of First-Year, First-Generation College Students from Ohio Appalachia. National Association of Student Personnel Administrators, 46(2), 258-281. 
Braxton, J.M. (2000). Introduction: Reworking the student departure puzzle. In J.M. Braxton (Ed.) Reworking the student departure puzzle (pp. 1-10). Nashville, TN: Vanderbilt University Press.

Braxton, J.M. \& Lien, L.A. (2000). The viability of academic integration as a central construct in Tinto's interactionalist theory of college student departure. In J.M. Braxton (Ed.) Reworking the student departure puzzle (pp. 11-28). Nashville, TN: Vanderbilt University Press.

Braxton, J.M., Sullivan, A.V.S., \& Johnson, R.M. (1997). Appraising Tinto's theory of college student departure. In J.C. Smart (Ed.), Higher Education: A handbook of theory and research, vol. 12 (pp. 107-164). New York, NY: Agathon Press.

Brown, L.M. \& Gilligan, C. (1992). Meeting at the crossroads: Women's psychology and girls' development. Cambridge, MA: Harvard University Press.

Bui, K.V.T. (2002). First-generation college students at a four-year university: Background characteristics, reasons for pursuing higher education, and first-year experiences. College Student Journal, 36(1), 3-12.

Butler, S.S., Deprez, L.S., \& Smith, R.J. (2004). Education: “The one factor that can keep me from sliding into hopeless poverty." Journal of Poverty, 8(2), 1-24. doi: 10.1300/J134v08n02_01

Campbell, R. \& Wasco, S.M. (2000). Feminist approaches to social science: Epistemological and methodological tenets. American Journal of Community Psychology, 28(6), 773-791. 
Carnevale, A.P., \& Strohl, J. (2010). How increasing college access is increasing inequality, and what to do about it. In R.D. Kahlenberg (Ed.), Rewarding strivers: Helping low-income students succeed in college (pp. 71-190). New York: Century Foundation Press.

Carter-Black, J. (2008). A black woman's journey into a predominately white academic world. Affilia, 23(2), 112-122.

Casstevens, W.J., Waites, C., \& Outlaw, N. (2012). Non-traditional student retention: Exploring perceptions of support in a social work graduate program. Social Work Education, 31(3), 256-268. doi: 10.1080/02615479.2011.556188

Chen, X. (2005). First Generation Students in Postsecondary Education: A Look at Their College Transcripts (NCES 2005-171). Retrieved from U.S. Department of Education, National Center for Education Statistics: http://nces.ed.gov/pubs2005/2005171.pdf

Choy, S. (2001). Students whose parents did not go to college: Post-secondary access, persistence, and attainment. (NCES 2001-126). Retrieved from U.S. Department of Education, National Center for Education Statistics: http://nces.ed.gov/pubs2001/2001126.pdf

Cohen, P.N. \& Huffman, M.L. (2003). Individual, jobs, and labor markets: The devaluation of women's work. American Sociological Review, 68(3), 443-463. Retrieved from: http://www.jstor.org/stable/1519732 
Cole, D. L. (2008). Staying within the margins: The educational stories of firstgeneration, low-income college students. Retrieved from ProQuest Dissertations and Theses. (3346833).

Coletrane, S. (2000). Research on Household Labor: Modeling and Measuring the Social Embeddedness of Routine Family Work. Journal of Marriage and the Family, 62, 1208-1233. Retrieved from: www.jstor.org/stable/1566732

Collier, P.J., \& Morgan, D.L. (2008). “Is that paper really due today?” Differences in first-generation and traditional college students' understandings of faculty expectations. Higher Education, 55, 425-446. doi: 10.1007/s10734-007-9065-5

Collins, P.H. (2000). Toward a politics of empowerment. In Black Feminist Thought: Knowledge, consciousness, and the politics of empowerment, (pp. 291-309). New York: Routledge.

Cook, T.D., \& Campbell, D.T. (1979). Quasi-Experimentation: Design and analysis issues for field settings. Chicago, IL: Rand McNally.

Council on Social Work Education. (2012). Annual Statistics on Social Work Education in the United States. Retrieved from Council on Social Work Education website: http://www.cswe.org/File.aspx?id=68989

Crenshaw, K.W. (1995). Mapping the margins: Intersectionality, identity politics, and violence against women of color. In K. Crenshaw, N. Gotanda, G. Peller and K. Thomas (Eds.) Critical Race Theory: The key writings that formed the movement. New York, NY: The New Press. 
Curry-Stevens, A., Cross-Hemmer, A., \& Coalition for Communities of Color. (2010). Communities of Color in Multnomah County: An Unsettling Profile. Portland, OR: Portland State University.

Daniel, C.A. (2007). Outsiders-Within: Critical race theory, graduate education, and barriers to professionalization. Journal of Sociology and Social Welfare, 34(1), $25-42$.

Davis, C.L. (2004). Black in the twenty-first century: A student's reflections on racial conflict in a BSW program. Reflections: Narratives of the Helping Profession, 96-102.

Day, J.C. \& Neuberger, E.C. (2002). The big payoff: Educational attainment and synthetic estimates of work-life earnings. Retrieved from http://smccd.net/accounts/wongk/Value\%20of\%20College.pdf

Delpit, L. (1995). Other people's children: Cultural conflict in the classroom. New York, NY: The New Press.

Deming, D. \& Dynarski, S. (2009). Into college, out of poverty? Policies to increase the postsecondary attainment of the poor. (Working paper 15387). Retrieved from National Bureau of Economic Research website: http://www.nber.org.proxy.lib.pdx.edu/papers/w15387.pdf

Denzin, N.K. \& Lincoln, Y.S. (2005). The discipline and practice of qualitative research. In N.K. Denzin \& Y.S. Lincoln (Eds.), The Sage Handbook of Qualitative Research (pp. 1-32). Thousand Oaks, CA: Sage. 
Eichler, M. (1986). The relationship between sexist, non-sexist, woman-centered, and feminist research. Studies in Communications, 3, 37-74.

Eichler, M. (1997). Feminist methodology. Current Sociology, 45(2), 9-36.

England, P., Allison, P., \& Wu, Y. (2007). Does bad pay cause occupations to feminize, does feminization reduce pay, and how can we tell with longitudinal data? Social Science Research, 36, 1237- 256. doi: 10.1016/j.ssresearch.2006.08.003

Engle, J., Bermeo, A., \& O'Brien, C. (2006). Straight from the source: What works for first-generation college students. Retrieved from The Pell Institute for the Study of Opportunity in Higher Education website: http://www.pellinstitute.org/publications-Straight_from_the_Source.shtml

Fine, M. (1992). Passions, politics, and power: Feminist research possibilities. In M. Fine (Ed.), Disruptive voices: The Possibilities of Feminist Research (pp. 205232). Ann Arbor, MI: University of Michigan Press.

Fine, M., \& Weis, L. (2005). Compositional studies, in two parts: Critical theorizing and analysis on social (in) justice. In N.K. Denzin and Y.S. Lincoln (Eds.), The Sage Handbook of Qualitative Research (pp. 65-84). Thousand Oaks, CA: Sage.

Frankland, J. \& Bloor, M. (1999). Some issues arising in the systematic analysis of focus group materials. In R.S. Barbour \& J. Kitzinger (Eds.), Developing focus group research: Politics, theory, and practice (pp. 144-155). London: Sage.

Freire, P. (1970/2000). Pedagogy of the oppressed. New York, NY: Continuum. 
Freire, P. (2005). Teachers as cultural workers: Letters to those who dare teach. Boulder, CO: Westview Press.

French, D. (2010, September 24). The college cost/benefit analysis. The National Review Online. Retrieved from: http://www.nationalreview.com/phi-beta$\underline{\text { cons/247749/college-cost-benefit-analysis-david-french }}$

Fry, R. (2012). Pew Research Center: A record one-in-five households now owe student loan debt. Retrieved from Pew Research Center website: https://www.cgsnet.org/ckfinder/userfiles/files/Pew-Student_Debt.pdf

Gamble, K., Huff, L., \& Jackson, A. (1973). For the love of money. On Ship Ahoy [LP]. Philadelphia, PA: Philadelphia International.

Gergen, K.J. (2009). Relational being: Beyond self and community. New York, NY: Oxford University Press.

Gofen, A. (2009). Family capital: How first-generation higher education students break the intergenerational cycle. Family Relations, 58, 104-120.

Giroux, H.A. (1983a). Theory and resistance in education. London: Heinemann Educational Books.

Giroux, H.A. (1983b). Theories of reproduction and resistance in the new sociology of education: A critical analysis. Harvard Educational Review, 53, 257-293.

Giroux, H.A. (1991). Modernism, postmodernism, and feminism: Rethinking the boundaries of educational discourse. In H.A. Giroux (Ed.), Postmodernism, 
RELATIONAL WORLDS OF FIRST-GEN STUDENTS

feminism, and cultural politics: Redrawing educational boundaries (pp. 1-59).

Albany, NY: State University of New York Press.

Giroux, H.A. (2001). Theory and resistance in education, $2^{\text {nd }}$ ed. Westport, CT: Bergin and Garvey.

Giroux, H.A. (2009). Education and the crisis of youth: Schooling and the promise of democracy. The Educational Forum, 73(1), 8-18. doi: $10 / 1080 / 00131720802539523$

Giroux, H.A. (2013, October 29). Public intellectuals against the neoliberal university. Truthout. Retrieved from: http://www.truth-out.org/opinion/item/19654-publicintellectuals-against-the-neoliberal-university

Gray, J., \& O’Neill, M. (2013). Social work students confront poverty’s impact in the community: A service-learning project. The Journal of Baccalaureate Social Work, 18, 217-232.

Gray, S.S. (2013). Framing "at-risk" students: Struggles at the boundaries of access to higher education. Children and Youth Services Review, 35, 1245-1251. doi: 10.1016/j.childyouth.2013.04.011

Guba, E.G. \& Lincoln, Y.S. (2005). Paradigmatic controversies, contradictions, and emerging confluences. In N.K. Denzin \& Y.S. Lincoln (Eds.) Sage Handbook of Qualitative Research (pp. 191-216). Thousand Oaks, CA: Sage.

Guiffrida, D.A. (2006). Toward a cultural advancement of Tinto's theory. The Review of Higher Education, 29(4), 451-472. 
Harding, S. (1993). Rethinking Standpoint Epistemology: What is "strong objectivity"? In L. Alcoff and E. Potter (Eds.), Feminist Epistemologies (pp. 49-82). New York, NY: Routledge.

Haveman, R., \& Smeeding, T. (2006). The role of higher education in social mobility. The Future of Children, 16(2), 125-150.

Hodges, J. Q. (2000). First-generation college students in an undergraduate social welfare program: Issues, interventions, and implications. The Journal of Baccalaureate Social Work, 6(1), 37-51.

hooks, b. (1994). Teaching to transgress: Education as the practice of freedom. New York, NY: Routledge.

hooks, b. (2000). Feminist theory: From margin to center. Cambridge, MA: South End Press.

Inkelas, K.K., Daver, Z.E., Vogt, K.E., \& Leonard, J.B. (2006). Living-learning programs and first-generation college students' academic and social transition to college. Research in Higher Education, 48(4), 403-434. doi: 10.1007/s11162006-9031-6

Jean, R. (2011, September 5). Bootstraps: Federal TRIO programs, if funded, could help close income gap. New England Journal of Higher Education, 1. Retrieved from: http://www.nebhe.org/thejournal/bootstraps-federal-trio-programs-iffunded-could-help-close-income-gap/ 
Johnson, V.D. (2010). Impact of race and welfare reform on African American single mothers' access to higher education. Journal of Black Studies, 40(6), 1041-1051. doi: $10.1177 / 0021934608323986$

Kamberelis, G. \& Dimitriadis, G. (2005). Focus groups: Strategic articulations of pedagogy, politics, and inquiry. In N.K. Denzin and Y.S. Lincoln (Eds.), The Sage Handbook of Qualitative Research (pp. 887-907). Thousand Oaks, CA: Sage.

Kapur, S. (2013, April 11). Is your college degree worth it? Find out. Forbes. Retrieved from: http://www.forbes.com/sites/saranyakapur/2013/04/11/is-your-collegedegree-worth-it-find-out/

Kincheloe, J.L. \& McLaren, P. (2005). Rethinking critical theory and qualitative research. In N.K. Denzin and Y.S. Lincoln (Eds.), The Sage Handbook of Qualitative Research (pp. 303-342). Thousand Oaks, CA: Sage.

Krueger, R.A. \& Casey, M.A. (2000). Focus groups: A practical guide for applied research. Thousand Oaks, CA: Sage.

Kuh, G.D. \& Love, P.G. (2000). A cultural perspective on student departure. In R.M. Braxton (Ed.), Reworking the student departure puzzle (pp. 196-212). Nashville, TN: Vanderbilt University Press.

Lather, P. \& Smithies, C. (1997). Troubling the angels: Women living with HIV/AIDS. Boulder, CO: Westview Press. 
Leotti, S. \& Muthanna, J. (2014). Troubling the Binary: A Critical Look at the Dualistic Construction of Quantitative/Qualitative Methods in Feminist Social Work Research. In S. Wahab, B. Anderson-Nathe, \& C. Gringeri (Eds.), Feminisms in Social Work Research: Promises and Possibilities for Justice Based Knowledge. (pp. 170-186). New York, NY: Routledge.

Levitas, R. (2004). Let's hear it for Humpty: Social exclusion, the third way, and cultural capital. Cultural Trends, 13(2), 41-56. doi: 10.1080/0954896042000267143

Lincoln, A.E. (2008). Gender, productivity, and the marital wage gap. Journal of Marriage and Family, 70(3), 806-814. Retrieved from: http://www.jstor.org/stable/40056369

Lincoln, Y.S. \& Guba, E. (1985) Naturalistic inquiry. Beverly Hills, CA: Sage

Liu, W.M. (2011). Social class and classism in the helping professions: Research, theory, and practice. Los Angeles, CA: Sage.

London, H.B. (1989). Breaking away: A study of first-generation college students and their families. American Journal of Education, 97(2), 144-170. Retrieved from: http://www.jstor.org/stable/1084908

London, H.B. (1992). Transformations: Cultural challenges faced by first-generation students. In L.S. Zwerling \& H.B. London (Eds.), First-generation college students: Confronting the cultural issues (pp. 5-12). Washington, D.C.: Office of Educational Research and Improvement. 
London, H.B. (1996). How college affects first-generation students. About Campus, 1(5), 9-13, 23. Retrieved from: http://eric.ed.gov/?id=EJ548703

London, R.A. (2006). The role of postsecondary education in welfare recipients' paths to self-sufficiency. The Journal of Higher Education, 77(3), 472-496.

Lowery-Hart, R. \& Pacheco, G. (2011). Understanding the first-generation student experience in higher education through a Relational Dialectic perspective. $\mathrm{New}$ Directions for Teaching and Learning, 127, 55-68. doi: 10.1002/t1.457

Lum, L. (2006). Handling "helicopter parents." Diverse: Issues in Higher Education, 23(20), 40-43. Retrieved from: http://diverseeducation.com/article/6657/

MacLeod, J. (1995). Ain't no makin' it: Aspirations and attainment in a low-income neighborhood. Boulder, CO: Westview Press.

Marshall, C. \& Rossman, G.B. (1999). Designing qualitative research. Thousand Oaks, CA: Sage.

McDonough, P.M. (1997). Choosing colleges: How schools and social class structure opportunity. Albany, NY: State University of New York Press.

Miller, R.L. (2013). Deconstructing the epistemological question with a focus on the knower. In A.E. Fortune, W.J. Reid \& R.L. Miller Jr. (Eds.), Qualitative Research in Social Work, (pp. 61-79). New York, NY: Columbia University Press. 
Mills, C.W. (1959). The sociological imagination. New York, NY: Oxford University Press.

Mirowsky, J. \& Ross, C.E. (2005). Education, cumulative advantage, and health. Ageing International, 30(1), 27-62.

Morris, T. (2006). Social work research methods: Four alternative paradigms. Thousand Oaks, CA: Sage.

National Association of Social Workers (2007). Institutional Racism and the Social Work Profession: A call to action. Retrieved from: http://www.socialworkers.org/diversity/institutionalracism.pdf

National Association of Social Workers (2008). Code of Ethics. Retrieved from: https://www.socialworkers.org/pubs/code/code.asp

National Center for Education Statistics. (2011). Median annual earnings of full-time, full-year wage and salary workers ages 25-34, by educational attainment and sex: Selected years 1980-2009. Retrieved from http://nces.ed.gov/fastfacts/display.asp?id=77

Noddings, N. (2002). Caring, social policy, and homelessness. Theoretical Medicine and Bioethics, 23(6), 441-454.

Oleson, V. (2005). Early millennial feminist qualitative research: Challenges and contours. In N.K. Denzin and Y.S. Lincoln (Eds.), The Sage Handbook of Qualitative Research (pp. 235-278. Thousand Oaks, CA: Sage. 
RELATIONAL WORLDS OF FIRST-GEN STUDENTS

Orbe, M.P. (2004). Negotiating multiple identities within multiple frames: An analysis of first-generation college students. Communication Education, 53(2), 131-149. doi:10.10/03634520410001682401

Padgett, D.K., Mathew, R., \& Conte, S. (2004). Peer debriefing and support groups: Formation, care, and maintenance. In D. Padgett (Ed.), The qualitative research experience (pp. 225-235). Belmont, CA: Thomson.

Padgett, R.D., Johnson, M.P., \& Pascarella, E.T. (2012). First-generation undergraduate students and the impact of the first year of college: Additional evidence. Journal of College Student Development, 53(2), 243-266. doi: 10.1353/csd.2012.0032

Paliadelis, P., \& Cruickshank, M. (2008). Using a Voice-Centered Relational Method of Data Analysis in a Feminist Study Exploring the Working World of Nursing Unit Managers. Qualitative Health Research, 18(10), 1444-1453. doi:

$10.1177 / 1049732308322606$

Pascarella, E.T., Pierson, C.T., Wolniak, G.C., \& Terenzini, P.T. (2004). First generation college students: Additional evidence on college experiences and outcomes. The Journal of Higher Education, 75(3), 249-284.

Perry, R. (2009). Factors influencing M.S.W. students' interest in clinical practice. Journal of Teaching in Social Work, 29(1), 47-70.

Preissle, J. \& Han, Y. (2012). Feminist research ethics. In S.N. Hesse-Biber (Ed.) Handbook of Feminist Research: Theory and praxis (pp. 583-605). Thousand Oaks, CA: Sage. 
Presser, L. (2005). Negotiating power and narrative in research: Implications for feminist methodology. Signs, 30(4), 2067-2090.

Rank, M.R. (2013, November 2). Poverty in America is mainstream. The New York Times. Retrieved from: http://opinionator.blogs.nytimes.com/2013/11/02/poverty-in-america-ismainstream/?_php=true\&_type=blogs\&_r=0

Reay, D. (2012). Future directions in difference research: Recognizing and responding to difference in the research process. In S.N. Hesse-Biber (Ed.), Handbook of Feminist Research: Theory and Praxis (pp. 627-640). Thousand Oaks, CA: Sage.

Reid, M.J., \& Moore III, J.L. (2008). College Readiness and Academic Preparation for Postsecondary Education Oral Histories of First-Generation Urban College Students. Urban Education, 43(2), 240-261. doi: 10.1177/0042085907312346

Rendon, L.I. (1992). From the barrio to the academy: Reflections of a MexicanAmerican “scholarship girl.” In L.S. Zwerling \& H.B. London (Eds.), Firstgeneration college students: Confronting the cultural issues (pp. 55-64). Washington, D.C.: Office of Educational Research and Improvement.

Renn, K.A. \& Reason, R.D. (2013). College students in the United States: Characteristics, Experiences, and Outcomes. San Francisco, CA: Jossey-Bass.

Richardson, R.C. \& Skinner, E.F. (1992). Helping first-generation minority students achieve degrees. New Directions for Community Colleges, 80, 29-43. doi: $10.1002 /$ cc. 36819928005 
Richardson, L. \& St. Pierre, E.A. (2005). Writing: A method of inquiry. In N.K. Denzin and Y.S. Lincoln (Eds.), The Sage Handbook of Qualitative Research (pp. 959978). Thousand Oaks, CA: Sage.

Richburg-Hayes, L. (2008). Helping low-wage workers persist in education programs: Lessons from research on welfare training programs and two promising community college strategies (MDRC Working Paper). Retrieved from MDRC website: http://www.mdrc.org/sites/default/files/helping_lowwage_workers_fr.pdf

Rosaldo, R. (1989). Culture and truth: The remaking of social analysis. Boston: Beacon Press.

Ryan, J. \& Sackrey, C. (1996). Strangers in paradise: Academics from the working class. Lanham, MD: University Press of America.

Saulnier, C.F. \& Swigonski, M. (2006). As feminists in the academy...Affilia: Journal of Women and Social Work, 21(4), 361-364. doi: 10.1177/0886109906292157

Seale, C. (2002). Quality issues in qualitative inquiry. Qualitative Social Work, 1(1), 97110.

Shoup, R., Gonyea, R.M., \& Kuh, G.D. (June, 2009). Helicopter parents: Examining the impact of highly involved parents on student engagement and educational outcomes. Paper presented at $49^{\text {th }}$ Annual Forum of the Association for Institutional Research, Atlanta, GA. Retrieved from: 
RELATIONAL WORLDS OF FIRST-GEN STUDENTS

http://cpr.iub.edu/uploads/AIR\%202009\%20Impact\%20of\%20Helicopter\%20Par

$\underline{\text { ents.pdf }}$

Smith, J.K. \& Hodkinson, P. (2005). Relativism, criteria, and politics. In N.K. Denzin and Y.S. Lincoln (Eds.), The Sage Handbook of Qualitative Research (pp. 915932). Thousand Oaks, CA: Sage.

Springer, K. (2002). Being the bridge: A solitary in Black Woman's position in the women's studies classroom as a feminist student and professor. In G.E. Anzaldúa \& A. Keating (Eds.). This bridge we call home: Radical visions for transformation (pp. 381-389). New York, NY: Routledge.

Stephens, N.M., Hamedani, M.G., Destin, M. (2014). Closing the social-class achievement gap: A difference-education intervention improves first-generation students' academic performance and all students' college transition. Psychological Science, 25(4), 943-953. doi: 10.117/0956797613518349

Strayhorn, T.L. (2006). Factors influencing the academic achievement of first generation college students. NASPA Journal, 43(4), 82-111.

Strega, S. (2005). The view from the post structural margins: Epistemology and methodology reconsidered. In L. Brown and S. Strega (Eds.), Research as resistance: Critical, indigenous, \& anti-oppressive approaches (pp. 199-236). Toronto, ON: Canadian Scholars' Press.

Stieha, V. (2010). Expectations and experiences: the voice of a first-generation first-year college student and the question of student persistence. International Journal of 
RELATIONAL WORLDS OF FIRST-GEN STUDENTS

Qualitative Studies in Education, 23(2), 237-249. Retrieved from:

http://dx.doi.org/10.1080/09518390903362342

Strier, R., Feldman, G., \& Shdaimah, C. (2012). The construction of social class in social work education: A study of introductory textbooks. Journal of Teaching in Social Work, 32(2), 406-420.

Stuber, J.M. (2011). Integrated, marginal, and resilient: race, class, and the diverse experiences of white first-generation college students. International Journal of Qualitative Studies in Education, 24(1), 117-136. Retrieved from: http://dx.doi.org/10.1080/09518391003641916

Tierney, W.G. (1999). Models of minority college going and retention: Cultural integrity versus cultural suicide. The Journal of Negro Education, 68(1), 80-91.

Tinto, V. (1975). Dropout from higher education: A theoretical synthesis of recent research. Review of Educational Research, 45(1), 89-125.

Tinto, V. (1993). Leaving college: Rethinking the causes and cures of student attrition. 2nd ed. Chicago, IL: University of Chicago Press.

Tugend, A. (2012, May 4). Vocation or exploration? Pondering the purpose of college. The New York Times. Retrieved from: http://www.nytimes.com/2012/05/05/your$\underline{\text { money/career-or-deep-learning-pondering-the-purpose-of- }}$ college.html?pagewanted=all 
RELATIONAL WORLDS OF FIRST-GEN STUDENTS

Terenzini, P.T., Springer, L., Yaeger, P.M., Pascarella, E.T., \& Nora, A. (1996). Firstgeneration college students: Characteristics, experiences and cognitive development. Research in Higher Education, 37(1), 1-22.

University Communications. (2014). Snapshot of Portland State [Public data]. Retrieved from: http://www.pdx.edu/profile/snapshot-portland-state

Van Galen, J. (2015, April 2). First gen students and assumptions of classlessness. [Web $\log$ comment]. Retrieved from: https://educationandclass.com/2015/04/02/firstgen-students-and-assumptions-of-classlessness/

Vandsburger, E., Duncan-Daston, R., Akerson, E., \& Dillon, T. (2010). The effects of poverty simulation, an experiential learning modality, on student understanding of life in poverty. Journal of Teaching in Social Work, 30(3), 300-316.

Wahab, S., Anderson-Nathe, B., \& Gringeri, C. (2012). Joining the conversation: Social work contributions to feminist research. In S. Hesse-Biber (Ed.) Handbook of Feminist Research, (pp. 455-474). Los Angeles, CA: Sage.

Wayne, R.H. (2013). Focus groups. In A.E. Fortune, W.J. Reid \& R.L. Miller Jr. (Eds.), Qualitative Research in Social Work, (pp. 264-283). New York, NY: Columbia University Press.

Weaver, R.D., \& Yun, S.H. (2011). Analyzing the attitude of undergraduate students toward poverty and impoverished persons: Does social work education make a difference. Journal of Teaching in Social Work, 31(1), 3-20. 
RELATIONAL WORLDS OF FIRST-GEN STUDENTS

Wiles, F. (2013). "Not easily put into a box": Constructing a professional social work identity. Social Work Education, 32(7), 854-866.

Wilkinson, S. (1999). How useful are focus groups in feminist research? In R.S.

Barbour \& J. Kitzinger (Eds.), Developing focus group research: Politics, theory, and practice (pp. 64-78). London: Sage.

Wink, J. (2005). Critical pedagogy: Notes from the real world. Boston, MA: Pearson.

Zosky, D.L., \& Thompson, J. (2012). Poverty simulation: An experiential learning tool emphasizing economic justice content. The Journal of Baccalaureate Social Work, 17, 69-84. 
Appendix A. 15 Propositions derived from Tinto’s 1975 Theoretical Schema

1. Student entry characteristics affect the level of initial commitment to the institution.

2. Student entry characteristics affect the level of initial commitment to the goal of graduation from college.

3. Student entry characteristics directly affect the student's likelihood of persistence in college.*

4. Initial commitment to the goal of graduation from college affects the level of academic integration.

5. Initial commitment to the goal of graduation from college affects the level of social integration.

6. Initial commitment to the institution affects the level of social integration.

7. Initial commitment to the institution affects the level of academic integration.

8. The greater the level of academic integration, the greater the level of subsequent commitment to the goal of graduation from college.*

9. The greater the level of social integration, the greater the level of subsequent commitment to the institution.*

10. The initial level of institutional commitment affects the subsequent level of institutional commitment.

11. The initial level of commitment to the goal of graduation from college affects the subsequent level of commitment to the goal of college graduation.

12. The greater the level of subsequent commitment to the goal of graduation, the greater the likelihood of student persistence in college.*

13. The greater the level of subsequent commitment to the institution, the greater the likelihood of student persistence in college.*

14. A high level of commitment to the goal of graduation from college compensates for a low level of commitment to the institution, and vice versa, in influencing student persistence in college.

15. A high level of academic integration compensates for a low level of social integration, and vice versa, in influencing student persistence in college. 
*These propositions were fundamental to the theory, as Tinto $(1975,1993)$ hypothesized that they had a direct influence on student departure decisions $(3,12$, and 13$)$ or were focused on integration, which is central to the theory $(8,9)$. 
Appendix B. Participant Consent Document

\section{Participant consent for participation in:}

\section{The relational worlds of first-generation students in a school of social work}

You are invited to participate in a research study conducted by Miranda Cunningham, a doctoral candidate from Portland State University's School of Social Work. The research is intended to describe the relational experiences of first-generation students in the school of social work. This study is being conducted by a student researcher under the guidance of Dr. Ben Anderson-Nathe (dissertation chair) from the Portland State University School of Social Work.

You were invited to participate in this study because of your experiences as a student for whom no parent and/or caregiver has completed a bachelor's degree. If you agree to participate in this study, you will be asked to participate in one 90-minute focus group discussions with other students in your program who are also "first-generation" students. These discussions will be audio recorded and may be videotaped for transcription purposes. While participating in this study, it is possible that you may feel uncomfortable or upset by one or more of the discussion questions or by responses shared by your fellow students. You may at any time decline to answer questions or end your participation in the discussion. It may be helpful to discover points of similarity with other students, but there is no guarantee of direct benefits to you from participation in this discussion. This study may increase knowledge which may help educators, students, and administrators invested in supporting the retention of first-generation students.

Because data will be collected during group discussions, we cannot promise complete confidentiality. For this reason it's important that you consider which stories you feel comfortable sharing with other students: which aspects of your relationships with family members, community, and within the school do you feel comfortable airing with students who you may share classes with, see in the halls, etc.? We will make every effort to maximize your confidentiality through the use of pseudonyms that you will select, and this pseudonym will be used in any reporting of data through publications or presentations. We will also maintain this consent form and digital recordings of the focus groups in a locked cabinet in the School of Social Work and in password-protected electronic files to minimize potential breaches of confidentiality. You will be invited to review, prior to publication, the preliminary results of analysis from these discussions, and your responses will be incorporated into final reports.

Participation in this study is completely voluntary. Deciding to participate in this study has no influence on your status as a student in the School of Social Work. You may also withdraw at any time without affecting your relationship with the School of Social Work. 
If you have concerns about participation in this study or your rights as a research participant, please contact the Human Subjects Research Review Committee, Office of Research and Strategic Partnerships, $1600 \mathrm{SW} 4^{\text {th }}$ Ave, Suite 620, Portland State University, (503) 725-3423 / 1-800-547-8887. If you have questions about the study itself, contact Miranda Cunningham at: School of Social Work, Portland State University, P.O. Box 751, Portland, Oregon, 97207; or by phone at (360) 904-2768.

Your signature indicates that you have read and understand the above information and agree to participate in this study. Please understand that you may withdraw your consent at any time without penalty, and that by signing, you are not waiving any legal claims, rights, or remedies. The researcher will provide you with a copy of this form for your own records.

Your name (printed)

Your signature

Date

preferred pseudonym

Yes, please contact me regarding de-briefing of preliminary study results in spring 2015.

You can reach me at:

(email address) 
Appendix C. Sample Recruitment flyer

So what does a social worker do, exactly?
What will you do with your degree when you're done with school?

\section{What's it like at a \\ university?}

- Are you the first in your family* to attend college?

- Are you interested in sharing your experiences of navigating relationships with family, members of your community, and relationships in school with other firstgeneration* students?

- Are you enrolled in the Bachelor of Social Work (BSW), Child and Family Studies (CFS), or Master of Social Work (MSW) program?

Miranda Cunningham is a doctoral student in the School of Social Work conducting an exploratory study of the relational experiences of first generation students in a school of social work, and will be conducting focus groups as part of her dissertation. You will receive $\$ 10$ as a thank you for your time and participation in group discussions with other students in your program. Group discussions will take no more than 90 minutes, and you'll have the opportunity to hear preliminary results during spring term.

If you're interested in joining in a group discussion, contact Miranda at miran2@pdx.edu

*folks have differing definitions of "first-generation," but for the purposes of this study, you're a first-generation student if none of your parents and/or caregivers completed a bachelor's degree. 
Appendix D. Focus Group Interview Questions

(main questions in bold, follow ups and probes are italicized) (introduction: approximately 20 minutes)

As you know, the main reason we're talking today is to learn more about the relational experiences of first-generation students in a school of social work. Before we begin, I want to remind everyone of the importance of protecting our family members' privacy and respecting each other's confidentiality. While I urge everyone to refrain from sharing other students' stories outside of this room, I can't prevent that, so I'd ask you to think carefully about which stories you share: which stories would you feel comfortable with other people hearing repeated in the school?

So to start with, I'd like you think about your identity in school, and jot down a few thoughts on your paper: How meaningful is your status as a first-generation student when you're at school? Perhaps there are examples of times when you were more aware of what it means to be the first in your family in school, or perhaps your awareness of this has changed throughout your time as a student.

In focus groups, not everyone is expected to talk equally, but for this first question, I'm hoping to hear from everyone, since this is something we have in common: we are all the first in our family to attend college.

(shifting to key questions: approximately 60 minutes)

Now I'd like you to shift to thinking about family: How meaningful is your identity as a student when you are with friends, family, or in your home community? What significance does your role as a student have in these familiar relationships, if any? Here it might be helpful to think about moments of separation or "translation" of your experiences for family members. How do you stay connected to family and community while also meeting the demands of being a student? How do you translate the experiences of school for your family, friends, and community?

Some people write about the distances between cultures of home and community and the culture of school. Have you experienced a sense of cultural difference between home and community and between the culture of school? If so, how do you navigate those cultural distances? What about spaces in the school that provide community? What are those spaces for you? Who is in those spaces, and what do they do to provide that sense of community?

How do family voices inform your ideas about what it means to be a social worker or a member of the helping profession (teacher, youth worker, counselor, early childhood educator, etc.)?

How do you think aspects of your identity (age, class, color, culture, ethnicity, gender, gender identity and/or expression, immigration status, race, religion, sex, and/or sexual orientation) have impacted our experiences as the first in your family to attend college? 
(wrap up: approximately 10 minutes) What advice would you give to other firstgeneration students in a school of social work about relationships in school, at home, and with members of their communities?

(facilitator summarizes general themes)

Does this sound like we covered everything? Is there anything you think we've missed? 
Appendix E: Listening guide, adapted from Brown and Gilligan (1992)

1. Who is speaking?

- what is the story the person tells? (geography of psychological landscape)

- plot? (points of narrative or "scheme")

- who, what, when, where, and why?

- what are the recurring words and images, metaphors, emotional resonances, contradictions or inconsistencies in style, revisions and absences in the story?

- where is the speaker in the narrative they tell? first ("I"), second ("you," "yours"), third ("he," "she," "it")

- what are my feelings and thoughts about the narrator and their story? how do I identify with this person or feel distant from this person?

- when am I confused or puzzled by their story; when am I certain about it?

- what are my reactions to the story: upset, delighted, amused, angered?

2. In what body?

- listen for the voice of the "I"...

- how does this person speak of themself (before I speak about them)

- note sentences with "I," "me," and "my"

3. Telling what story about relationship: from whose perspective or from what vantage point?

- note struggles for relationships that are authentic or resonant: in which they can speak thoughts and feelings and be heard

- when are relationships used to distance, psychologically violate, subordinate, invalidate, or oppress? and when are relationships used to encourage, free, and empower? (especially in terms of gender stereotypes) [here remember the experience/reality split]

- when do speakers self-silence or self-sacrifice?

- when do women strive for purity and perfection (i.e. the "good girl") and men for domination and control, self-aggrandizement

- how do speakers describe relationships between social identity groups?

- what about interactions in the room? how do participants construct a sense of self for and with the others in the room?

\section{In what societal and cultural frameworks?}

- listen for self-silencing in capitulation to cultural norms and values (confusion, uncertainty, disassociation) 
- what about moral voices that silence, constrain, and narrow relationships? (and what are institutionalized or cultural norms and values behind these)

- or political resistance? ability to disagree openly, feel and speak a full range of emotions

- how do experiences fit into the broader society? 